\title{
A DIFFERENTIAL GENE EXPRESSION ALGORITHM FOR COMPARATIVE MICROARRAY ANALYSIS
}

\author{
by \\ John L. Houle

\begin{abstract}
A thesis submitted to
the Faculty of Graduate Studies and Research

in partial fulfillment of

the requirements for the degree of

DOCTOR OF PHILOSOPHY

School of Computer Science

CARLETON UNIVERSITY
\end{abstract} \\ Ottawa-Carleton Institute for Computer Science
}

Ottawa, Ontario

May, 2008

(C) Copyright by John L. Houle, 2008 


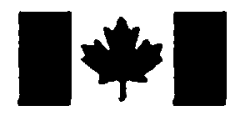

\author{
Library and Archives \\ Canada \\ Published Heritage \\ Branch \\ 395 Wellington Street \\ Ottawa ON K1A ON4 \\ Canada
}

Bibliothèque et

Archives Canada

Direction du

Patrimoine de l'édition

395 , rue Wellington

Ottawa ON K1A ON4

Canada
Your file Votre référence
ISBN: 978-0-494-63851-4
Our file Notre référence
ISBN: 978-0-494-63851-4
NOTICE:

The author has granted a nonexclusive license allowing Library and Archives Canada to reproduce, publish, archive, preserve, conserve, communicate to the public by telecommunication or on the Internet, loan, distribute and sell theses worldwide, for commercial or noncommercial purposes, in microform, paper, electronic and/or any other formats.

The author retains copyright ownership and moral rights in this thesis. Neither the thesis nor substantial extracts from it may be printed or otherwise reproduced without the author's permission.
AVIS:

L'auteur a accordé une licence non exclusive permettant à la Bibliothèque et Archives Canada de reproduire, publier, archiver, sauvegarder, conserver, transmettre au public par télécommunication ou par l'Internet, prêter, distribuer et vendre des thèses partout dans le monde, à des fins commerciales ou autres, sur support microforme, papier, électronique et/ou autres formats.

L'auteur conserve la propriété du droit d'auteur et des droits moraux qui protège cette thèse. $\mathrm{Ni}$ la thèse ni des extraits substantiels de celle-ci ne doivent être imprimés ou autrement reproduits sans son autorisation.
In compliance with the Canadian Privacy Act some supporting forms may have been removed from this thesis.

While these forms may be included in the document page count, their removal does not represent any loss of content from the thesis.
Conformément à la loi canadienne sur la protection de la vie privée, quelques formulaires secondaires ont été enlevés de cette thèse.

Bien que ces formulaires aient inclus dans la pagination, il n'y aura aucun contenu manquant. 


\section{Abstract}

The novel Differential Gene Expression (DGE) algorithm for the comparative analysis of microarrays presented here provided a powerful method for the determination of changes in gene expression (both upregulation and downregulation) under control and experimental conditions. The DGE algorithm which used the Nonlinear Fold-Change (NFC) method for assessing changes in gene expression was shown to improve upon two types of algorithms: 1. precedent differential gene expression algorithms which used the Linear Fold-Change (LFC) method and 2. Significance Analysis of Microarrays (SAM) algorithm which used the modified T-statistic method. The NFC method which was based on nonparametric quantile regression analysis was implemented using the kernel weighted local linear fitting algorithm to approximate conditional quantile functions of gene expression. The nonparametric quantile regression analysis was used for the estimation of the gene ranks. The generation of the more stable gene rank estimates led to the more effective identification of differential gene expression. The SAM algorithm and the DGE algorithm were subjected to comparative accuracy performance analysis and comparative precision performance analysis. The DGE algorithm offered five distinct advantages over the SAM algorithm: 1. increased accuracy, 2. increased precision, 3. automated differential criterion selection, 4. increased statistical gene expression measurement categories and 5. more robust statistical gene expression measurement classification error fault tolerance. While the DGE algorithm represented an improvement over the current SAM algorithm, the DGE algorithm nevertheless demonstrated four limitations: 1. terminal quantile computation, 2. terminal expression level measurement computation, 3. local neighborhood computation and 4. time complexity analysis. Five future research directions for extending the DGE algorithm were proposed: 1. exploration of statistical gene expression measurement properties, 2. development of an extended nonparametric quantile regression algorithm, 3. development of an extended local neighborhood algorithm, 4. parallel computer implementation and 5. calculation of receiver operating characteristic curves. 


\section{Table of Contents}

$\begin{array}{ll}\text { Abstract } & \text { ii }\end{array}$

Table of Contents $\quad$ iii

Abbreviations vii

List of Tables $\quad$ x

List of Figures $\quad$ xii

Acknowledgements xiv

Chapter 1 Introduction 1

1.1 Differential Gene Expression (DGE) Problem 1

1.2 Current Solution Limitations 3

1.3 Doctoral Thesis Contributions $\quad 8$

1.4 Doctoral Thesis Organization $\quad 12$

Chapter 2 Literature Review 14

$\begin{array}{lll}2.1 & \text { Microarray Technologies } & 15\end{array}$

2.2 Normalization Algorithms for Comparative Microarray Analysis 16

2.3 Differential Gene Expression Algorithms for Comparative Microarray 18 Analysis

2.4 Significance Analysis of Microarrays (SAM) Algorithm 20

$2.5 \quad$ Regression analysis 26 
Chapter 3 Differential Gene Expression (DGE) Algorithm 30

3.1 Differential Gene Expression (DGE) Algorithm Strategy 31

3.2 Input Data and Output Data 32

3.3 Normalization Algorithm 35

3.4 Nonparametric Quantile Regression Algorithm 37

3.4.1 Quantile Initialization Algorithm 40

3.4.2 Median Algorithm 41

3.4.3 Kernel Localization Function 41

3.4.4 $\mathrm{S}_{\mathrm{n}, \mathrm{l}}$ Function $\quad 42$

3.4.5 Weight Function $\quad 42$

3.4.6 Matrix Inversion Algorithm 43

3.4.7 Residual Squares Criterion Weight Function 43

3.4.8 Residual Squares Criterion Algorithm 43

3.4.9 $\mathrm{H}_{1, \text { minimum present }}$ Algorithm $\quad 46$

$\begin{array}{lll}\text { 3.4.10 } & \mathrm{H}_{1, \text { expansion-contraction Algorithm }} & 47\end{array}$

3.4.11 $\mathrm{H}_{1, \text { mean }}$ Algorithm $\quad 53$

3.4.12 $\mathrm{H}_{1, \mathrm{p}}$ Algorithm $\quad 54$

3.4.13 $\mathrm{H}_{2, \mathrm{p}}$ Algorithm $\quad 55$

3.4.14 Local Linear Kernel Estimation Function 55

3.4.15 Implicit Estimation Function $\quad 56$

3.4.16 Quantile Tolerance Function 57

3.4.17 Nonparametric Quantile Regression Algorithm 57

3.5 Differential Gene Expression (DGE) Algorithm Logical Structure 58

Chapter 4 Experimental Analysis $\quad 69$

4.1 Synthetic Microarray Datasets Method 71

4.1.1 Synthetic Upregulated Genes Method 72

4.1.2 Synthetic Downregulated Genes Method 74

$\begin{array}{lll}4.2 & \text { Confusion Matrix Classification Statistics } & 76\end{array}$

4.3 Comparative Accuracy Performance Analysis 80 
$\begin{array}{lll}\text { 4.3.1 Statistical Procedure } & 80\end{array}$

$\begin{array}{lll}\text { 4.3.2 } & \text { Microarray Datasets } & 84\end{array}$

4.3.3 Significance Analysis of Microarrays (SAM) Algorithm 90

4.3.4 Significance Analysis of Microarrays (SAM) Fixed Array Method 93 Accuracy Performance Analysis

4.3.5 Differential Gene Expression (DGE) Algorithm 94

4.3.6 Differential Gene Expression (DGE) Fixed Array Method Accuracy 97 Performance Analysis

4.3.7 Differential Gene Expression (DGE) Fixed Quantile Method Accuracy 99 Performance Analysis

4.3.8 Differential Gene Expression (DGE) Differential Maximum Exclusion 100 Method Accuracy Performance Analysis

4.3.9 Differential Gene Expression (DGE) Differential Maximum Inclusion 102 Method Accuracy Performance Analysis

4.3.10 Differential Gene Expression (DGE) Differential Maximum Inclusion 105 Method Accuracy Performance Analysis Using the Confusion Matrix Classification System

4.4 Comparative Precision Performance Analysis

4.4.1 Statistical Procedure $\quad 112$

$\begin{array}{lll}\text { 4.4.2 Microarray Datasets } & 114\end{array}$

4.4.3 Significance Analysis of Microarrays (SAM) Algorithm 116

4.4.4 Differential Gene Expression (DGE) Algorithm 119

4.4.5 Statistical Results 123

$\begin{array}{ll}\text { Chapter } 5 \text { Discussion } & 128\end{array}$

5.1 Comparative Statistical Gene Expression Measurement Properties 129

5.1.1 Significance Analysis of Microarrays (SAM) Algorithm Statistical 129 Gene Expression Measurement Properties

5.1.2 Differential Gene Expression (DGE) Algorithm Statistical Gene 131 Expression Measurement Properties

5.2 Comparative Differentiation Mechanisms 
5.2.1 Significance Analysis of Microarrays (SAM) Algorithm Differentiation 138 Mechanism

$\begin{array}{llllll}\text { 5.2.2 Differential Gene Expression (DGE) Algorithm Differentiation } & 139\end{array}$ Mechanism

$\begin{array}{lll}5.3 & \text { Comparative Analysis } & 140\end{array}$

5.4 Differential Gene Expression (DGE) Algorithm Limitations 141

Chapter 6 Conclusion $\quad 144$

6.1 Differential Gene Expression (DGE) Algorithm Summary 145

6.1.1 Differential Gene Expression (DGE) Algorithm 145

$\begin{array}{lll}\text { 6.1.2 Comparative Analysis } & 146\end{array}$

6.1.3 Differential Gene Expression (DGE) Algorithm Limitations 147

6.1.4 Differential Gene Expression (DGE) Algorithm Significance 148

6.2 Differential Gene Expression (DGE) Algorithm Future Research 148 Directions

Glossary

References 


\section{Abbreviations}

\begin{tabular}{|c|c|}
\hline Abbreviation & Term \\
\hline abs & absolute \\
\hline $\mathrm{AC}$ & accuracy \\
\hline $\mathrm{C}$ & class \\
\hline $\mathrm{CF}$ & class frequency \\
\hline DGE & Differential Gene Expression \\
\hline DNA & deoxyribonucleic acid \\
\hline DUQR & downregulated / upregulated quantile ratio \\
\hline $\mathrm{e}$ & natural logarithm base \\
\hline $\mathrm{E}$ & expected \\
\hline FD & found down \\
\hline FDP & found down proportion \\
\hline FDR & false discovery rate \\
\hline FM & F-measure \\
\hline FN & false negative \\
\hline FNR & false negative rate \\
\hline FP & false positive \\
\hline FPR & false positive rate \\
\hline FU & found up \\
\hline FUP & found up proportion \\
\hline $\mathrm{F} 1 \mathrm{M}$ & $F_{1}$-measure \\
\hline GEO & Gene Expression Omnibus \\
\hline GM1 & geometric-mean $_{1}$ \\
\hline GM2 & geometric-mean $_{2}$ \\
\hline I & identity function \\
\hline LB & lower boundary \\
\hline $\mathrm{LFC}$ & Linear Fold-Change \\
\hline LOESS & locally-weighted regression and smoothing scatterplots \\
\hline
\end{tabular}




\begin{tabular}{ll} 
LOWESS & locally-weighted regression and smoothing scatterplots \\
LLB & loge $_{\mathrm{e}}$ lower boundary \\
LUB & $\log _{\mathrm{e}}$ upper boundary \\
MAX & maximum \\
MD & median \\
MIN & minimum \\
MN & mean \\
mRNA & messenger ribonucleic acid \\
MTI & Molecular Target Identification \\
NCBI & National Center for Biotechnology Information \\
NFC & Nonlinear Fold-Change \\
NPV & negative predictive value \\
P & power \\
PD & predicted down \\
Ph.D. & Doctor of Philosophy \\
PPV & positive predictive value \\
PR & precision \\
PU & predicted up \\
Q1 & first quantile \\
Q3 & third quantile \\
RE & recall \\
ROC & receiver operating characteristic \\
RSC & residual squares criterion \\
SAM & Significance Analysis of Microarrays \\
SE & sensitivity \\
SP & specificity \\
sqrt & square root \\
TDR & total discovery rate \\
TN & true negative \\
TNR & true negative rate \\
TP & true positive \\
\hline
\end{tabular}




$\begin{array}{ll}\text { TPR } & \text { true positive rate } \\ \text { UB } & \text { upper boundary } \\ \text { UDQR } & \text { upregulated / downregulated quantile ratio } \\ \phi & \text { standard normal density function } \\ \Phi & \text { standard normal distribution function } \\ \alpha & \text { Type I error rate } \\ \beta & \text { Type II error rate } \\ \# & \text { experiment number }\end{array}$




\section{List of Tables}

$\begin{array}{lll}\text { Table } 1 . \quad \text { Confusion matrix. } & 76\end{array}$

Table 2. Significance Analysis of Microarrays (SAM) algorithm fixed array 93 method accuracy performance analysis.

Table 3. Differential Gene Expression (DGE) algorithm fixed array method 97 accuracy performance analysis.

Table 4. Differential Gene Expression (DGE) algorithm fixed quantile 99 method accuracy performance analysis.

Table 5. Differential Gene Expression (DGE) algorithm differential 100 maximum exclusion method accuracy performance analysis.

Table 6. Differential Gene Expression (DGE) algorithm differential 102 maximum inclusion method accuracy performance analysis.

Table 7a. Differential Gene Expression (DGE) algorithm differential 105 maximum inclusion method accuracy performance analysis using the confusion matrix classification system for the upregulated genes.

Table 7b. Differential Gene Expression (DGE) algorithm differential 106 maximum inclusion method accuracy performance analysis using the confusion matrix classification system for the upregulated genes.

Table 8a. Differential Gene Expression (DGE) algorithm differential 107 maximum inclusion method accuracy performance analysis using the confusion matrix classification system for the downregulated genes.

Table 8b. Differential Gene Expression (DGE) algorithm differential 108 maximum inclusion method accuracy performance analysis using the confusion matrix classification system for the downregulated genes.

Table 9a. Differential Gene Expression (DGE) algorithm differential 109 
maximum inclusion method accuracy performance analysis using the confusion matrix classification system for the upregulated genes and the downregulated genes.

Table 9b. Differential Gene Expression (DGE) algorithm differential 110 maximum inclusion method accuracy performance analysis using the confusion matrix classification system for the upregulated genes and the downregulated genes.

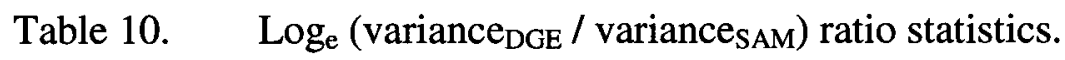

123

Table 11. $\log _{\mathrm{e}}\left(\right.$ variance $_{\mathrm{DGE}} /$ variance $_{\mathrm{SAM}}$ ) ratio frequency distribution. 


\section{List of Figures}

Figure 1. Linear Fold-Change (LFC) method including the two parallel linear 5 fold-change boundaries which represent the upper linear fold-change threshold and the lower linear fold-change threshold.

Figure 2. Nonlinear Fold-Change (NFC) method including the two nonparallel 10 nonlinear fold-change boundaries which represent the upper nonlinear fold-change threshold and the lower nonlinear fold-change threshold.

Figure 3. Differential gene expression graph. 23

Figure 4. Significance Analysis of Microarrays (SAM) algorithm total 24 discovery rate (TDR) graph and false discovery rate (FDR) graph.

Figure 5. Significance Analysis of Microarrays (SAM) algorithm difference 25 statistic graph and expected difference statistic graph.

Figure 6. Nonparametric quantile regression algorithm call tree.

$\begin{array}{lll}\text { Figure 7. Differential gene expression graph. } & 64\end{array}$

Figure 8. Differential Gene Expression (DGE) algorithm nonlinear regression 65 quantile graphs.

Figure 9. Differential Gene Expression (DGE) algorithm quantile graph and 66 expected quantile graph.

Figure 10. Differential Gene Expression (DGE) algorithm nonlinear regression 0.03 quantile graph and 0.97 quantile graph.

Figure 11. Differential Gene Expression (DGE) algorithm graph. 68

Figure 12. Differential Gene Expression (DGE) algorithm graph including the nonlinear regression 0.01 quantile graph and the nonlinear regression 0.99 quantile graph.

Figure 13. Differential Gene Expression (DGE) algorithm graph including the nonlinear regression 0.01 quantile graph and the nonlinear regression 0.99 quantile graph.

Figure 14. Differential Gene Expression (DGE) algorithm graph including the 
nonlinear regression 0.01 quantile graph and the nonlinear regression 0.99 quantile graph.

Figure 15. Differential Gene Expression (DGE) algorithm graph including the 88 nonlinear regression 0.01 quantile graph and the nonlinear regression 0.99 quantile graph.

Figure 16. Differential Gene Expression (DGE) algorithm graph including the nonlinear regression 0.01 quantile graph, the nonlinear regression 0.05 quantile graph, the nonlinear regression 0.95 quantile graph and the nonlinear regression 0.99 quantile graph.

Figure 17. Significance Analysis of Microarrays (SAM) algorithm total 91 discovery rate (TDR) graph and false discovery rate (FDR) graph.

Figure 18. Significance Analysis of Microarrays (SAM) algorithm difference statistic graph and expected difference statistic graph.

Figure 19. Differential Gene Expression (DGE) algorithm quantile graph and expected quantile graph.

Figure 20. Differential Gene Expression (DGE) algorithm quantile graph and 96 revised expected quantile graph.

Figure 21. Differential gene expression graph.

Figure 22. Significance Analysis of Microarrays (SAM) algorithm total discovery rate (TDR) graph and false discovery rate (FDR) graph.

Figure 23. Significance Analysis of Microarrays (SAM) algorithm difference statistic graph and expected difference statistic graph.

Figure 24. Differential Gene Expression (DGE) algorithm nonlinear regression quantile graphs.

Figure 25. Differential Gene Expression (DGE) algorithm quantile graph and expected quantile graph.

Figure 26. Differential Gene Expression (DGE) algorithm quantile graph and 122 revised expected quantile graph.

Figure 27. $\log _{\mathrm{e}}\left(\right.$ variance $_{\mathrm{DGE}} /$ variance $_{\mathrm{SAM}}$ ) ratio histogram. 


\section{Acknowledgements}

The following individuals and institutions are gratefully acknowledged for having contributed to the successful completion of my Doctor of Philosophy (Ph.D.) degree in computer science at Carleton University. The School of Computer Science at Carleton University and the Faculty of Graduate Studies and Research at Carleton University provided me with the opportunity to engage in doctoral study and earn my Ph.D. degree in computer science at Carleton University. Dr. Nicola Santoro, Dr. Daniel Krewski and Dr. Timothy Ramsay were my doctoral thesis supervisors. Dr. Santoro and Dr. Krewski provided me with scientific mentorship, financial support and moral encouragement throughout my doctoral candidacy. Dr. Ramsay posed the open research problem which formed the basis for the doctoral thesis research. Dr. Ramsay also provided me with invaluable mathematical assistance during the algorithmic solution for the doctoral thesis research. Dr. Santoro, Dr. Krewski and Dr. Ramsay were the perfect doctoral thesis advisors. Dr. Prosenjit Bose, Dr. Jean-Pierre Corriveau, Sandy Herbert, Jane MacArthur, Sharmi Namasivayampillai, Linda Pfeiffer and Claire Ryan were the administrative staff responsible for the administration of graduate students in the School of Computer Science at Carleton University. Peter Choynowski, John MacGillivray, Andrew Miles and Gerardo Reynaga were the technical staff responsible for the successful computer operations in the School of Computer Science at Carleton University. Dr. Peter Taillon was the fellow Ph.D. candidate in the School of Computer Science at Carleton University who acted as a sounding board for my research ideas during numerous brainstorming sessions. Dr. Taillon also acted as a guide during the completion of the landmark milestone requirements for my Ph.D. degree. Suzanne Therien was the directorial assistant responsible for the administration of graduate students in the R. Samuel McLaughlin Centre for Population Health Risk Assessment at the University of Ottawa. Arthur Kealey, James Gillespie and Scott Cameron from Allegra Print and Imaging prepared the electronic versions of the figures. Mohr Davidow Ventures and the Ontario Government jointly granted me the Ontario Graduate Scholarship in Science and Technology in the academic year 2001-2002. Jeffrey Dale at the Ottawa Centre for Research and Innovation granted me the Futures Award for the Student Researcher of the 
Year in the academic year 2002. The R. Samuel McLaughlin Centre for Population Health Risk Assessment at the University of Ottawa and the Ontario Government jointly granted me the Ontario Graduate Scholarship in Science and Technology in the academic year 2004-2005. The Faculty of Graduate Studies and Research at Carleton University granted me the Doctoral Dissertation Award in the academic year 2004-2005. Dr. Nicola Santoro, Dr. Daniel Krewski, Dr. Timothy Ramsay, Dr. Franz Oppacher, Dr. Amiyaranjan Nayak, Dr. James Cheetham, Dr. Dennis Bulman and Dr. Michel Gaulin formed the doctoral thesis committee. I would further like to thank the doctoral thesis committee for the examination of this doctoral thesis. Finally, I would especially like to thank my parents Lorne Houle and Shirley Houle who provided me with unwavering financial support, moral encouragement and sources of inspiration throughout my many years of university study as I strove to attain my lifelong ambition of earning a Ph.D. degree. This doctoral thesis is dedicated to the memory of the Avro Arrow, the aeronautical engineering technology by which Canada put the United States on the moon. 


\section{Chapter 1 Introduction}

\subsection{Differential Gene Expression (DGE) Problem}

The Molecular Target Identification (MTI) problem was first encountered in the field of rational drug design. The MTI problem was defined as the discovery of the molecular targets amenable to pharmaceutical intervention in order to prevent, stabilize or reverse diseases. MTI was the critical first stage in the multistage process of pharmaceutical research and development. MTI provided the motivation for the development of the differential gene expression experiments. MTI examined the differential gene expression of the messenger ribonucleic acid (mRNA) molecules in two cases: 1. normal cells versus pathological cells and 2. pathological cells versus pharmaceutically-treated pathological cells. Microarray technologies were used to identify the alterations in the gene expression measurements. MTI assumed that the gene expression measurements of the relevant molecular targets were altered in the pathological cells or following the pharmaceutical treatments. In the MTI problem, the crucial step was the classification of the gene expression measurements into three categories: 1. positive regulation genes, 2. neutral regulation genes and 3. negative regulation genes. MTI in rational drug design has been reviewed (Jayapal and Melendez 2006).

The differential gene expression experiments led to the recognition of the Differential Gene Expression (DGE) problem. The DGE problem was formally defined as the identification of the genes exhibiting differential expression under the alternate experimental conditions. The DGE problem has been extensively studied in precedent research because differential gene expression formed the essential component in MTI. Differential gene expression experiments were conducted using pairwise samples with the first set of samples corresponding to the control state and the second set of samples corresponding to the experimental state. Based on the response to the alternate experimental conditions, microarray datasets were generated under the control state condition and under the experimental state condition. The control state microarray 
datasets and the experimental state microarray datasets were subjected to differential gene expression algorithms for comparative analysis which have been implemented as computational software. The differential gene expression algorithms compared the control state microarray datasets to the experimental state microarray datasets. The DGE problem involved the categorization of the differences between the control state gene transcription measurements and the experimental state gene transcription measurements.

The categorization of the differences between the control state gene transcription measurements and the experimental state gene transcription measurements resulted in three possible gene transcription classifications: 1. positive regulation, 2. neutral regulation and 3. negative regulation. The positive regulation case signified that the control state gene transcription measurements were smaller than the experimental state gene transcription measurements. The positive regulation classification was indicative of induced gene transcription. The neutral regulation case signified that the control state gene transcription measurements were equal to the experimental state gene transcription measurements. The neutral regulation classification was indicative of unchanged gene transcription. The negative regulation case signified that the control state gene transcription measurements were larger than the experimental state gene transcription measurements. The negative regulation classification was indicative of repressed gene transcription.

Differential gene expression experiments addressed two objectives: 1 . variance identification and 2. experimental correlation. In variance identification, the qualitative set of genes exhibiting the most statistically-significant variation between the control samples and the experimental samples was selected for further data analysis. In experimental correlation, the quantitative variation in the transcriptional production of the mRNA molecules was correlated with the specific experimental conditions. The DGE problem arose in six fields of contemporary scientific research: 1. MTI in rational drug design (Jayapal and Melendez 2006), 2. identification of genes critical in stem cell differentiation or developmental processes (Aiba et al. 2006), 3. description of genes mediating cellular responses to toxic chemical or radiation physical stimuli (Gatzidou et 
al. 2007; National Research Council 2007; Ogawa et al. 2007), 4. reverse engineering of pathological mechanisms arising from complex genetic diseases (Nanni et al. 2006), 5. definition of diagnostic biomarkers for complex genetic diseases and pharmaceutical research (He 2006) and 6. classification of pharmacogenomic events associated with pharmaceutical treatments (Villeneuve and Parissenti 2004).

\subsection{Current Solution Limitations}

An extensive number of differential gene expression algorithms have been developed for the comparative analysis of microarrays. Differential gene expression algorithms for comparative microarray analysis are discussed in section 2.3. The majority of the differential gene expression algorithms were based on the strategy of applying fixed gene expression ratios for the identification of differentially-expressed genes in comparative microarray analysis. Fold changes were calculated as the ratio between the experimental expression measurements and the control expression measurements for each gene. Nondifferentially-expressed genes exhibited fold changes which were grouped around unity.

Differential gene expression algorithms were based on the strategy which we referred to as the Linear Fold-Change (LFC) method. The LFC method used two parallel linear fold-change boundaries which were chosen as the criteria to identify the set of genes exhibiting the most statistically-significant variation between the control samples and the experimental samples. Logarithmic gene expression measurements for the experimental samples were plotted against logarithmic gene expression measurements for the control samples using a scatterplot of logarithmically-transformed gene expression measurements rearranged as Cartesian coordinates (Figure 1). The choice of the unit gene expression ratio for distinguishing differential gene expression corresponded to the nondifferentially-expressed genes which approximated $y=0$. The choice of the upper linear fold-change threshold was represented as the parallel straight line at $y>0$ of uniform slope 0 . Gene expression measurements which deviated above the upper linear fold-change limit were classified as positive differentially-expressed genes. The choice 
of the lower linear fold-change threshold was represented as the parallel straight line at $y$ $<0$ of uniform slope 0 . Gene expression measurements which deviated below the lower linear fold-change limit were classified as negative differentially-expressed genes (Draghici 2002). 


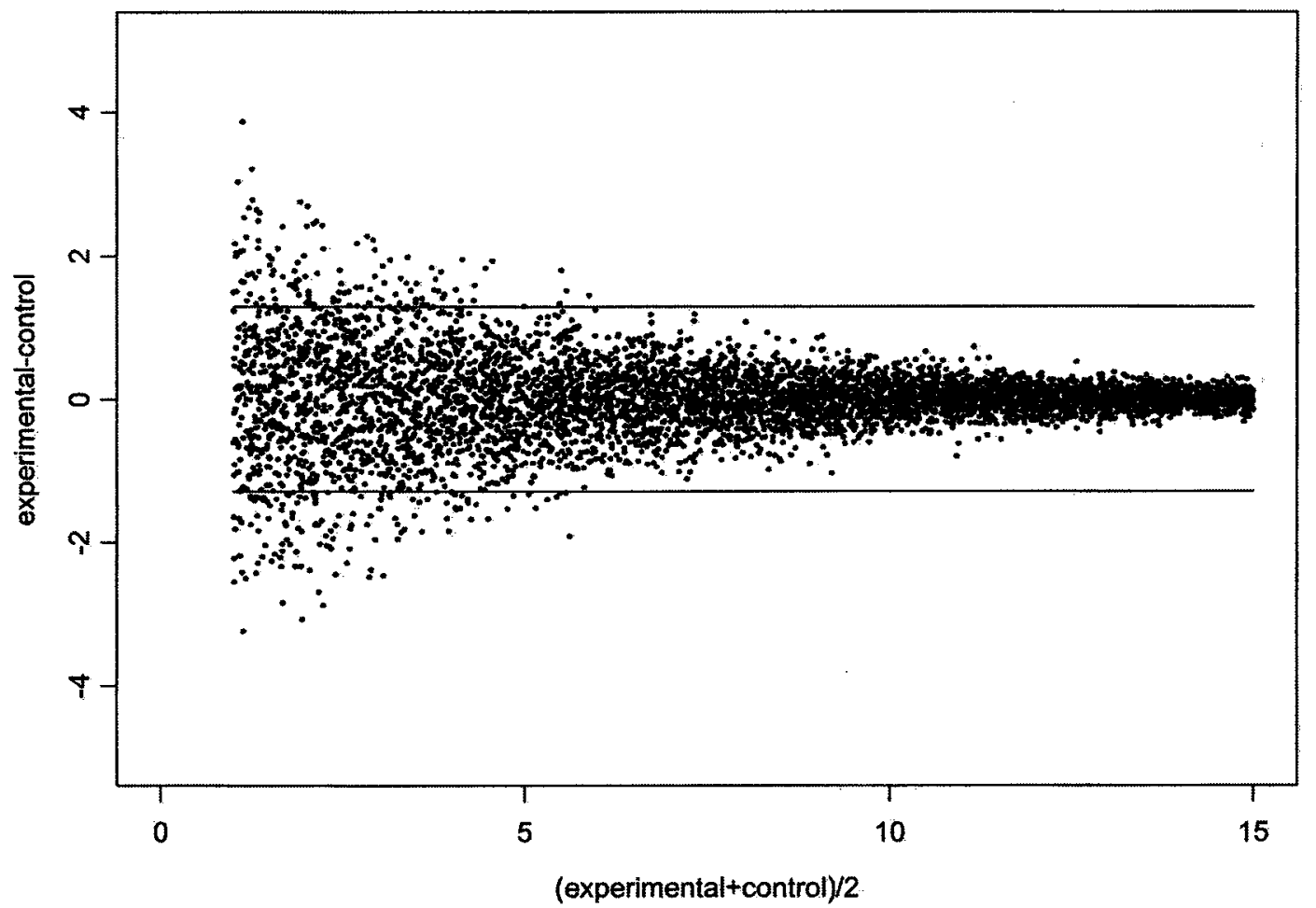

Figure 1. Linear Fold-Change (LFC) method including the two parallel linear fold-change boundaries which represent the upper linear fold-change threshold and the lower linear fold-change threshold. 
The LFC method demonstrated two significant deficiencies: 1. arbitrary foldchange limits and 2. nonuniform signal variance. With arbitrary fold-change limits, the linear fold-change boundaries were not based on rigorous biological criteria and were chosen arbitrarily. Most published research used a postnormalization gene expression ratio of two-fold (increase or decrease) in the transcription measurements to define differential gene expression. There was no rigorous theoretical basis for selecting this linear fold-change threshold as significant. The LFC method considered genes with large changes in differential expression corresponding to at least a two-fold increase or a twofold decrease in the transcription measurements to be significant. The arbitrary choice of nonunit gene expression ratios for identifying differential gene expression introduced divergence between the upper linear fold-change threshold, the microarray dataset and the lower linear fold-change threshold.

The adoption of at least a two-fold increase in transcription measurements for recognizing differential gene expression corresponded to a logarithmically-transformed upper linear fold-change boundary at $y=\log (2)=0.69315$ of uniform slope 0 . The adoption of at least a two-fold decrease in transcription measurements for recognizing differential gene expression corresponded to a logarithmically-transformed lower linear fold-change boundary at $y=-\log (2)=-0.69315$ of uniform slope 0 .

The introduction of divergence between the upper linear fold-change threshold, the microarray dataset and the lower linear fold-change threshold disrupted the inclusive nature of the upper linear fold-change threshold and the lower linear fold-change threshold. Divergence between the upper linear fold-change threshold, the microarray dataset and the lower linear fold-change threshold complicated the accurate identification of small changes in the differential gene expression. The LFC method adopted conservative linear fold-change thresholds for differential gene expression with the result that the false positives or type I errors for reporting differential gene expression were low. The conservative linear fold-change boundaries produced under-representation rather than over-representation of the differential gene expression. The LFC method failed to distinguish the genes with small though biologically-significant changes in the 
differential expression from experimental noise. The arbitrary choice of the linear foldchange thresholds was biologically unsuitable and amounted to at best an intuitive guess.

With nonuniform signal variance, microarray technologies demonstrated low signal-to-noise ratios for the genes with low expression levels. There was an inverse correlation between the signal intensity levels and the signal variance. In the scatterplot, the gene expression measurements were visualized as a funnel-shaped distribution. Gene expression measurements at low levels exhibited large variance while gene expression measurements at high levels exhibited small variance. At low expression levels, nondifferentially-expressed genes might have been misclassified as differentiallyexpressed genes thereby missing true negatives and introducing false positives. These type I errors reduced the specificity of the LFC method. At high expression levels, differentially-expressed genes might have been misidentified as nondifferentiallyexpressed genes thereby missing true positives and introducing false negatives. These type II errors reduced the sensitivity of the LFC method. The funnel-shaped distribution of the scatterplot suggested that the parallel straight lines representing the linear foldchange limits did not accurately reflect the inherent structure of the microarray datasets. Thus, the LFC method was inadequate for the development of differential gene expression algorithms (Draghici 2002).

The Significance Analysis of Microrrays (SAM) algorithm was the differential gene expression algorithm which adopted the completely different strategy from the LFC method. The SAM algorithm was based on the modified T-statistic. The SAM algorithm formed the most advanced comparative datamining algorithm for the solution of the DGE problem to date (Tusher et al. 2001, 2002). The modified T-statistic demonstrated one significant deficiency: the modified T-statistic was computed on the basis of the individual genes. The individual gene computations would have rendered the modified T-statistic unstable for small gene sample sizes. The modified T-statistic estimated the individual gene ranks and identified the differential gene expression on the basis of the gene ranks. If the gene ranks were not being reliably estimated, then it would not have 
been possible to reliably detect the differential gene expression. Thus, the modified Tstatistic was inadequate for the development of differential gene expression algorithms.

\subsection{Doctoral Thesis Contributions}

The primary contribution of the doctoral thesis has been the invention of the novel Differential Gene Expression (DGE) algorithm which was developed as the more efficient alternative comparative datamining algorithm for the solution of the Differential Gene Expression (DGE) problem.

The novel normalization algorithm formed the preliminary step of the DGE algorithm. The normalization algorithm was developed for the standardization of the signals in the control state microarray datasets and the experimental state microarray datasets to comparable levels of intensity. Transformed microarray datasets compensated for the systematic variations as a prerequisite to allowing the meaningful comparative microarray analysis between the control microarray datasets and the experimental microarray datasets in the differential gene expression algorithms.

Unlike the precedent differential gene expression algorithms, the DGE algorithm was based on the alternate strategy which we referred to as the Nonlinear Fold-Change (NFC) method. The NFC method used two nonparallel nonlinear fold-change boundaries which were chosen as the criteria to identify the set of genes exhibiting the most statistically-significant variation between the control samples and the experimental samples. Logarithmic gene expression measurements for the experimental samples were plotted against logarithmic gene expression measurements for the control samples using a scatterplot of logarithmically-transformed gene expression measurements rearranged as Cartesian coordinates (Figure 2). The choice of the unit gene expression ratio for distinguishing differential gene expression corresponded to the nondifferentiallyexpressed genes which approximate $y=0$. The choice of the upper nonlinear foldchange threshold was represented as the nonparallel curved line at $y>0$ of nonuniform increasing slope $<0$. Gene expression measurements which deviated above the upper 
nonlinear fold-change limit were classified as positive differentially-expressed genes. The choice of the lower nonlinear fold-change threshold was represented as the nonparallel curved line at $\mathrm{y}<0$ of nonuniform decreasing slope $>0$. Gene expression measurements which deviated below the lower nonlinear fold-change limit were classified as negative differentially-expressed genes.

The funnel-shaped distribution of the scatterplot suggested that the nonparallel curved lines representing the nonlinear fold-change limits accurately reflected the inherent structure of the microarray datasets (Draghici 2002). The NFC method would have theoretically corrected the two deficiencies of the Linear Fold-Change (LFC) method. Thus, the DGE algorithm which adopted the NFC method was predicted by us to be more accurate than the current differential gene expression algorithms which were based on the LFC method.

The NFC method was based on the nonparametric quantile regression analysis. The nonparametric quantile regression analysis was implemented using the kernel weighted local linear fitting algorithm to approximate conditional quantile functions ( $\mathrm{Yu}$ and Jones 1998). The nonparametric quantile regression analysis was used for the estimation of the gene ranks. The nonparametric quantile regression ranks possessed two advantages: 1 . probability distribution and 2 . rank stability. The nonparametric quantile regression ranks did not require the assumption of the Gaussian probability distribution. The nonparametric quantile regression ranks pooled information from all of the genes with similar expression levels to compute more stable gene rank estimates. The second advantage was particularly important under the limited conditions that existed when the gene sample sizes were small. The generation of the more stable gene rank estimates would have led to the more effective identification of the differential gene expression. The more stable gene rank estimates would have theoretically corrected the one deficiency of the modified T-statistic. Thus, the DGE algorithm which adopted the nonparametric quantile regression analysis was predicted by us to be more accurate than the Significance Analysis of Microarrays (SAM) algorithm which was based on the modified T-statistic. 


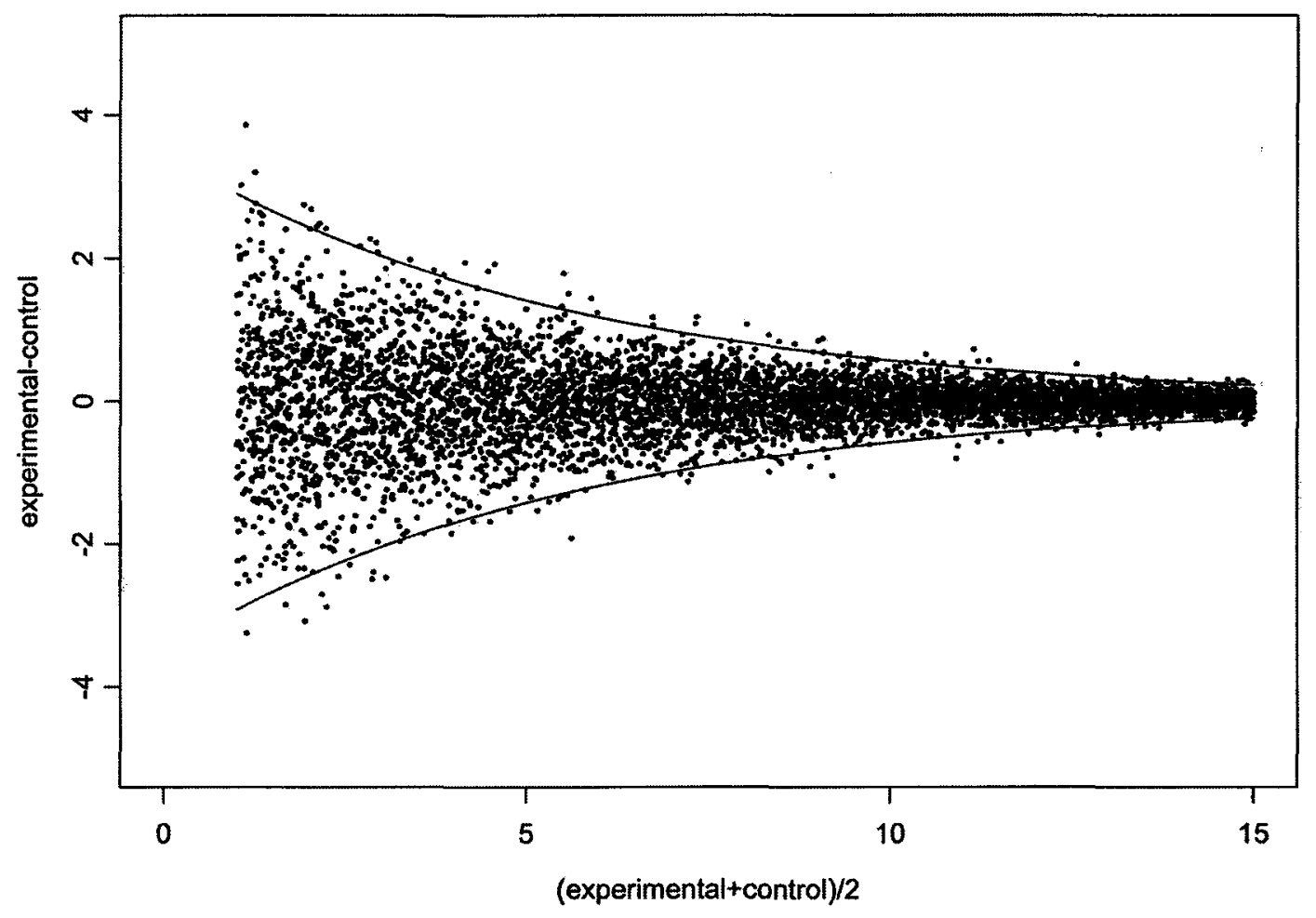

Figure 2. Nonlinear Fold-Change (NFC) method including the two nonparallel nonlinear fold-change boundaries which represent the upper nonlinear fold-change threshold and the lower nonlinear fold-change threshold. 
The DGE algorithm was based on the use of quantiles as the statistical gene expression measurements. The quantiles demonstrated three statistical gene expression measurement properties: 1 . nonsymmetric crossover effect, 2 . nonsymmetric differential effect and 3. differential fallback effect.

The DGE algorithm used the following differentiation mechanism for the classification of the statistical gene expression measurements into the different categories. The statistical gene expression measurements were based on quantiles. The quantiles were sorted on the basis of ascending quantile order. The two differential criteria were chosen by taking advantage of the differential fallback effect through the utilization of the differential maximum inclusion method. Human intervention was not necessary. The two differential criteria corresponded to the maximum positive differential $\mathrm{d}_{\mathrm{mp}}$ and the maximum negative differential $\mathrm{d}_{\mathrm{mn}}$. The maximum positive differential $d_{m p}$ was used indirectly for the selection of the maximum positive differential quantile $q_{m p}$. The maximum negative differential $d_{m n}$ was used indirectly for the selection of the maximum negative differential quantile $\mathrm{q}_{\mathrm{mn}}$. The maximum positive differential quantile $\mathrm{q}_{\mathrm{mp}}$ and the maximum negative differential quantile $\mathrm{q}_{\mathrm{mn}}$ were used directly as the decision factors for the classification of the quantiles into three statistical gene expression measurement categories: 1. downregulated genes, 2 . nonregulated genes and 3. upregulated genes. The quantile classifications were expanded to include three statistical gene expression measurement categories: 1. downregulated genes, 2 . nonregulated genes and 3. upregulated genes. The statistical gene expression measurement categories differentiated between the downregulated genes and the upregulated genes. The quantile classifications were resistant to the statistical gene expression measurement classification errors caused by two statistical gene expression measurement properties: 1. nonsymmetric crossover effect and 2. nonsymmetric differential effect.

The proof-of-concept experiments provided convincing evidence that the SAM algorithm was surpassed by the DGE algorithm which offered five distinct advantages over the SAM algorithm: 1. accuracy performance analysis, 2. precision performance 
analysis, 3. differential criterion selection, 4. statistical gene expression measurement categories and 5. statistical gene expression measurement classification error fault tolerance. The DGE algorithm offered increased accuracy, increased precision and employed an automated differential criterion selection. The DGE algorithm provided an increased number of more detailed statistical gene expression measurement categories. The DGE algorithm demonstrated more robust statistical gene expression measurement classification error fault tolerance. The proof-of-concept experiments provided convincing evidence that the SAM algorithm has been superseded by the DGE algorithm as the more efficient alternative comparative datamining algorithm for the solution of the DGE problem.

The proof-of-concept experiments provided convincing evidence that the DGE algorithm is the more efficient alternative comparative datamining algorithm for the solution of the DGE problem. Because of its uses in other applications, we can foresee that the DGE algorithm will advance six fields of contemporary scientific research: 1 . molecular target identification in rational drug design (Jayapal and Melendez 2006), 2. identification of genes critical in stem cell differentiation or developmental processes (Aiba et al. 2006), 3. description of genes mediating cellular responses to toxic chemical or radiation physical stimuli (Gatzidou et al. 2007; National Research Council 2007; Ogawa et al. 2007), 4. reverse engineering of pathological mechanisms arising from complex genetic diseases (Nanni et al. 2006), 5. definition of diagnostic biomarkers for complex genetic diseases and pharmaceutical research (He 2006) and 6. classification of pharmacogenomic events associated with pharmaceutical treatments (Villeneuve and Parissenti 2004).

\subsection{Doctoral Thesis Organization}

The remaining five chapters of the doctoral thesis were organized as follows. In chapter 2, we reviewed the scientific literature on five subjects: 1. microarray technologies, 2. normalization algorithms for comparative microarray analysis, 3 . differential gene expression algorithms for comparative microarray analysis, 4. 
Significance Analysis of Microarrays (SAM) algorithm and 5. regression analysis. In chapter 3, we advanced the Differential Gene Expression (DGE) algorithm in five design specifications: 1. DGE algorithm strategy, 2. formats of the input data and the output data for the Differential Gene Expression (DGE) problem, 3. novel normalization algorithm for the standardization of the signals in the control state microarray datasets and the experimental state microarray datasets to comparable levels of intensity, 4. customized nonparametric quantile regression algorithm and 5. novel DGE algorithm for the solution of the DGE problem. In chapter 4, we conducted the experimental analysis on the relative performance of the SAM algorithm and the DGE algorithm using four statistical procedures: 1 . methods used to generate the synthetic microarray datasets containing known differentially-expressed genes, 2. performance statistics used to evaluate the confusion matrix for the binary classification system used in the accuracy performance experiments, 3. accuracy performance experiments of the SAM algorithm and the DGE algorithm and 4. precision performance experiments of the SAM algorithm and the DGE algorithm. In chapter 5, we hypothesized theoretical models of the SAM algorithm and the DGE algorithm using four aspects: 1. statistical gene expression measurement properties of the SAM algorithm and the DGE algorithm, 2. differentiation mechanisms of the SAM algorithm and the DGE algorithm, 3. advantages of the DGE algorithm over the SAM algorithm and 4. limitations of the DGE algorithm. In chapter 6, we concluded the doctoral thesis with two topics: 1. summary of the DGE algorithm and 2. proposed future research directions for the DGE algorithm. 


\section{Chapter 2 Literature Review}

The Differential Gene Expression (DGE) algorithm was based on the extension of previous statistical research. The previous statistical research relevant for the development of the DGE algorithm was presented using the literature review. The literature review surveyed the scientific literature on five topics: 1. microarray technologies, 2. normalization algorithms for comparative microarray analysis, 3 . differential gene expression algorithms for comparative microarray analysis, 4. Significance Analysis of Microarrays (SAM) algorithm and 5. regression analysis.

Microarray technologies were defined as high-throughput analytical devices that provided comprehensive genome-scale expression analysis by the simultaneous monitoring of quantitative transcription measurements of thousands of genes in parallel.

Normalization algorithms for comparative microarray analysis eliminated the systematic biases between the raw microarray datasets by the systematic error adjustments to the individual hybridization intensities. Large-scale systematic biases in the signal intensities between the control microarray datasets and the experimental microarray datasets were corrected using the normalization algorithms which rescaled or standardized the gene expression measurements relative to each other. Transformed microarray datasets compensated for the systematic variations as a prerequisite to allowing the meaningful comparative microarray analysis between the control microarray datasets and the experimental microarray datasets in the differential gene expression algorithms.

Differential gene expression algorithms for comparative microarray analysis have been devised in response to the Differential Gene Expression (DGE) problem. Microarray datasets were generated under the control state condition and under the experimental state condition. The control state microarray datasets and the experimental state microarray datasets were subjected to differential gene expression algorithms for comparative analysis which have been implemented as computational software. The 
differential gene expression algorithms classified the statistical gene expression measurements into three categories: 1. downregulated genes, 2. nonregulated genes and 3. upregulated genes. Current differential gene expression algorithms demonstrated limited performance capabilities which motivated the development of more advanced differential gene expression algorithms.

The SAM algorithm was presented in the detailed design specifications. The detailed design specifications formed the basis for the implementation of the SAM algorithm as computational software. The SAM algorithm computational software performance formed the reference benchmark against which the DGE algorithm computational software performance was compared. Competition between the SAM algorithm and the DGE algorithm formed the basis for the comparative performance analysis experiments.

Regression analysis was defined as a mathematical technique that formulated a mathematical model of the relationship between a set of $\mathrm{q}$ independent variables $\mathrm{x}_{1}, \mathrm{x}_{2}, \mathrm{x}_{3}$ $\ldots x_{q}$ and a dependent variable $y$. The DGE algorithm was based on the Nonlinear FoldChange (NFC) method. The NFC method was based on the nonparametric quantile regression analysis. The nonparametric quantile regression analysis was implemented using the kernel weighted local linear fitting algorithm to approximate conditional quantile functions ( $\mathrm{Yu}$ and Jones 1998).

\subsection{Microarray Technologies}

Microarray technologies were defined as high-throughput analytical devices that provided comprehensive genome-scale expression analysis by the simultaneous monitoring of quantitative transcription measurements of thousands of genes in parallel. Microarray technologies were based on two principles: 1. transcriptional variation and 2 . complementary sequence hybridization. Transcriptional variation referred to the fact that gene expression was primarily regulated at the transcriptional level. The predominant factor underlying gene expression alteration was quantitative variation in the 
transcriptional production of messenger ribonucleic acid (mRNA) molecules. Complementary sequence hybridization referred to the fact that only deoxyribonucleic acid (DNA) single-stranded molecules possessing complementary sequences were capable of mutual hybridization for the formation of stable DNA double-stranded molecules. Microarrays immobilized millions of single-stranded copies of each gene as individual array elements onto a solid support surface. During the hybridization reaction, the microarray surface was incubated with a mixture of labeled DNA molecules. The microarray surface contained a proportional representation of the set of all the genes that were being expressed in the experimental sample. Only the subset of labeled DNA molecules that represented the same genes corresponding to the immobilized DNA sequences could form heteroduplexes. Following the completion of the hybridization reaction, the measurement of the amount of label bound to each microarray element revealed the relative transcript abundance quantity corresponding to each gene. Microarrays contained thousands of elements which enabled the simultaneous monitoring of quantitative transcription measurements of thousands of genes in parallel. Microarray technologies were differentiated on the basis of their DNA sequence probe lengths into two types: 1. oligonucleotide microarrays and 2. DNA microarrays. Oligonucleotide microarrays used small synthetic DNA molecules approximately 20 nucleotides long as DNA sequence probes. DNA microarrays used large natural gene fragments from 400 to 2000 nucleotides long as DNA sequence probes. Microarray technologies have been reviewed (Elvidge 2006; Hager 2006; Hardiman and Carmen 2006; Hoheisel 2006; Taylor 2006; Vo-Dinh 2006; Mandruzzato 2007; Petersen and Kawasaki 2007).

\subsection{Normalization Algorithms for Comparative Microarray Analysis}

Systematic sources of error occurred in the gene expression measurements between the comparative microarray experiments. Systematic bias corrupted the microarray data quality by introducing false artifacts which did not reflect the true differences in the transcriptional production of the messenger ribonucleic acid (mRNA) molecules. Systematic bias was attributable to the differences in eight factors: 1. array surface chemistry, 2 . hybridization parameters, 3 . image analysis, 4 . labeling methods, 5 . 
microarray printing, 6. mRNA isolation, 7. nucleic acid quantities present in target preparation and 8. washing efficiency. Systematic errors in the comparative microarray analysis have been reviewed (Finkelstein et al. 2002). Microarray data quality could have been enhanced by improvements in five experimental steps: 1 . nucleotide sequence selection, 2. microarray design, 3. experimental design, 4. experimental annotation and 5. empirical result verification.

Normalization algorithms were further available for the detection, quantification and removal of the large-scale systematic biases. Large-scale systematic biases in the signal intensities between the control samples and the experimental samples were corrected using the normalization algorithms which rescaled or standardized the gene expression measurements relative to each other. Normalization algorithms eliminated the systematic biases between the raw microarray datasets by the systematic error adjustments to the individual hybridization intensities. Normalization algorithms were composed of two sequential steps: 1. selection and calibration of invariant genes and 2 . estimation of a normalization constant or function. In the selection and calibration of the invariant genes, a group of nondifferentially-expressed genes known to not be affected by the alternate experimental conditions under investigation were first identified. Invariant genes were chosen using five strategies: 1. global normalization, 2. housekeeping genes, 3. exogenous control genes, 4. genomic deoxyribonucleic acid (DNA) and 5. algorithmic selection.

Global normalization used the entire set of the signal intensities from each experiment as the reference set. The global method assumed that only a small proportion of the genes represented on a microarray exhibited differential expression. In comparing the two complete datasets, the systematic variation was assumed to be equal to the overall variation measured between the two datasets. The datasets were normalized by multiplying each signal by a factor that was inversely proportional to the overall variation. Global normalization was the recommended general approach. Housekeeping genes were assumed to generate constant transcription and to not exhibit differential expression under the alternate experimental conditions. Exogenous control genes used 
the heterologous control mRNAs with no sequence homologies to the mRNAs from the samples under investigation. Equal quantities of mRNA samples corresponding to the heterologous control genes were added to both the control samples and the experimental samples. The normalization factor was then determined by comparison of the signal intensities of the heterologous control genes in the control samples and the experimental samples. Genomic DNA was reasoned to represent a constant copy number for a given mass of DNA.

Algorithmic selection of the invariant genes could have been estimated using mathematical criteria. Rank-invariant algorithms selected the signal intensities from the spots where the difference of the rank of the control signals and the experimental signals were very close and where the rank of the spots was not within the highest or the lowest overall ranks. While the absolute signal intensities could have been different due to the systematic errors, their ranks within the entire group were similar which indicated no change.

In the estimation of a normalization constant or function, the linear or the nonlinear normalization for the signal intensities over the signal intensity range was chosen using the selected set of invariant genes. Transformed microarray datasets compensated for the systematic variations as a prerequisite to allowing the meaningful comparative microarray analysis between the control samples and the experimental samples in the differential gene expression algorithms. Normalization algorithms for comparative microarray analysis have been reviewed (Bilban et al. 2002; Bobashev et al. 2002; Finkelstein et al. 2002; Quackenbush 2002; Wolkenhauer et al. 2002; Aittokallio et al. 2003; Benes and Muckenthaler 2003; Sartor et al. 2003; Saviozzi and Calogero 2003; Kreil and Russell 2005; Reimers 2005; Zhang et al. 2005; Do and Choi 2006; Parrish and Delongchamp 2006; Steinhoff and Vingron 2006; Yang 2006).

\subsection{Differential Gene Expression Algorithms for Comparative Microarray Analysis}


Gene expression research was defined as the use of the quantitative messenger ribonucleic acid (mRNA) measurements of gene transcription in order to characterize biological processes and to elucidate the mechanisms of gene transcription. The objective of gene expression research was the quantitative measurement of the mRNA expression particularly under the influence of drug perturbations or disease perturbations. High-throughput analysis of gene expression was facilitated by microarray technologies. Gene expression experiments used microarray technologies for the quantitative mRNA measurements. The identification of the differential gene expression associated with biological processes was a central research problem in molecular biology.

Microarray datasets were generated under the control state condition and under the experimental state condition. The control state microarray datasets and the experimental state microarray datasets were subjected to differential gene expression algorithms for comparative analysis which have been implemented as computational software.

The differential gene expression algorithms classified the statistical gene expression measurements into three categories: 1. downregulated genes, 2. nonregulated genes and 3. upregulated genes. Differential gene expression experiments addressed two objectives: 1. variance identification and 2. experimental correlation. In variance identification, the qualitative set of genes exhibiting the most statistically-significant variation between the control samples and the experimental samples were selected for further data analysis. In experimental correlation, the quantitative variation in the transcriptional production of the mRNA molecules was correlated with specific experimental conditions.

Transcriptional profiling experiments demonstrating significant differential gene expression addressed six problems in functional genomics: 1. molecular target identification in rational drug design (Jayapal and Melendez 2006), 2. identification of genes critical in stem cell differentiation or developmental processes (Aiba et al. 2006), 3. description of genes mediating cellular responses to toxic chemical or radiation physical 
stimuli (Gatzidou et al. 2007; National Research Council 2007; Ogawa et al. 2007), 4. reverse engineering of pathological mechanisms arising from complex genetic diseases (Nanni et al. 2006), 5. definition of diagnostic biomarkers for complex genetic diseases and pharmaceutical research (He 2006) and 6. classification of pharmacogenomic events associated with pharmaceutical treatments (Villeneuve and Parissenti 2004). The largescale investigation of differential gene expression attached functional activity to structural genomics and therefore was an essential milestone in the paradigm shift from static structural genomics to dynamic functional genomics.

The Significance Analysis of Microrrays (SAM) algorithm was based on the modified T-statistic. The SAM algorithm formed the most advanced comparative datamining algorithm for the solution of the Differential Gene Expression (DGE) problem (Tusher et al. 2001, 2002). A recent differential gene expression algorithm was based on the rank score test for linear quantile regression models with a random effect (Wang and He 2007). Current differential gene expression algorithms demonstrated limited performance capabilities which motivated the development of more advanced differential gene expression algorithms. Differential gene expression algorithms for comparative microarray analysis have been reviewed (Vietor and Huber 1997; Carulli et al. 1998; Claverie 1999; Vedoy et al. 1999; Frohme et al. 2000; Chen et al. 2001; Bobashev et al. 2002; Chen et al. 2002; Draghici 2002; Krajewski and Bocianowski 2002; Li and Yang 2002; Nadon and Shoemaker 2002; Pan 2002; Sabatti 2002; Slonim 2002; Aittokallio et al. 2003; Cui and Churchill 2003; Dobbin et al. 2003; Hariharan 2003; Hatfield et al. 2003; Kerns et al. 2003; Kramer 2003; Stolovitzky 2003; Churchill 2004; Hackl et al. 2004; Saviozzi et al. 2004; Tadesse and Ibrahim 2004; Yang et al. 2004; Goldstein and Delorenzi 2005; Reimers 2005; Zhang et al. 2005; Ayroles and Gibson 2006; Chu et al. 2006; Edwards and Ghosh 2006; Larsson et al. 2006; Liang and Kelemen 2006; Neal and Westwood 2006; Steinhoff and Vingron 2006; Gusnanto et al. 2007; Roesok and Sioud 2007).

\subsection{Significance Analysis of Microarrays (SAM) Algorithm}


As described in the original publications, the Significance Analysis of Microrrays (SAM) algorithm was based on the modified T-statistic. The SAM algorithm formed the most advanced comparative datamining algorithm for the solution of the Differential Gene Expression (DGE) problem (Tusher et al. 2001, 2002). The SAM algorithm subjected the gene transcription measurements in the three control state microarray datasets and the three experimental state microarray datasets to a series of five calculations:

1. The difference statistics were computed using the modified T-statistic.

2. The expected difference statistics were computed as the average difference statistics arising from the $C(6,3)=20$ combinations of the six microarray datasets consisting of the three control microarray datasets and the three experimental microarray datasets.

3. The differentials were computed as the absolute differences between the difference statistics and the expected difference statistics: for $\mathrm{i}=1$ to $\mathrm{n}, \mathrm{d}_{\mathrm{i}}=\operatorname{abs}\left(E\left(\mathrm{~s}_{\mathrm{i}}\right)-\mathrm{s}_{\mathrm{i}}\right)$.

4. The differentials were sorted into ascending order.

5. The differentials greater than the specified differential criterion were classified as differentially-expressed gene transcription measurements.

The total discovery rate (TDR) was the percentage of differentials that were identified as differentially-expressed gene transcription measurements using the specified differential criterion. The false discovery rate (FDR) was the percentage of differentials that were incorrectly identified by random chance as differentially-expressed gene transcription measurements using the specified differential criterion. The FDR was estimated by the identification of nonsense genes in the $C(6,3)=20$ combinations of the six microarray datasets consisting of the three control microarray datasets and the three experimental microarray datasets. The TDR graph visualized the systematic investigation of the TDR output in response to variation in the differential criterion input. 
The FDR graph visualized the systematic investigation of the FDR output in response to variation in the differential criterion input. The differential criterion was an adjustable value which offered competing tradeoffs between the TDR and the FDR. The optimal differential criterion was chosen by the user on the basis of consultation with the TDR graph and the FDR graph.

The SAM algorithm was demonstrated using synthetic microarray datasets. The synthetic microarray datasets underwent artificial generation, log transformation, Cartesian coordinate calculation and normalization. The synthetic microarray datasets consisted of 0.03 normalized fraction upregulated genes, 0.94 normalized fraction neutral genes and 0.03 normalized fraction downregulated genes. Refer to the differential gene expression graph (Figure 3). The synthetic microarray datasets were subjected to the SAM algorithm computation. The SAM algorithm computation performed intermediate calculations which underwent graphical visualization. Refer to the SAM algorithm TDR graph and FDR graph (Figure 4). Refer to the SAM algorithm difference statistic graph and expected difference statistic graph (Figure 5). 


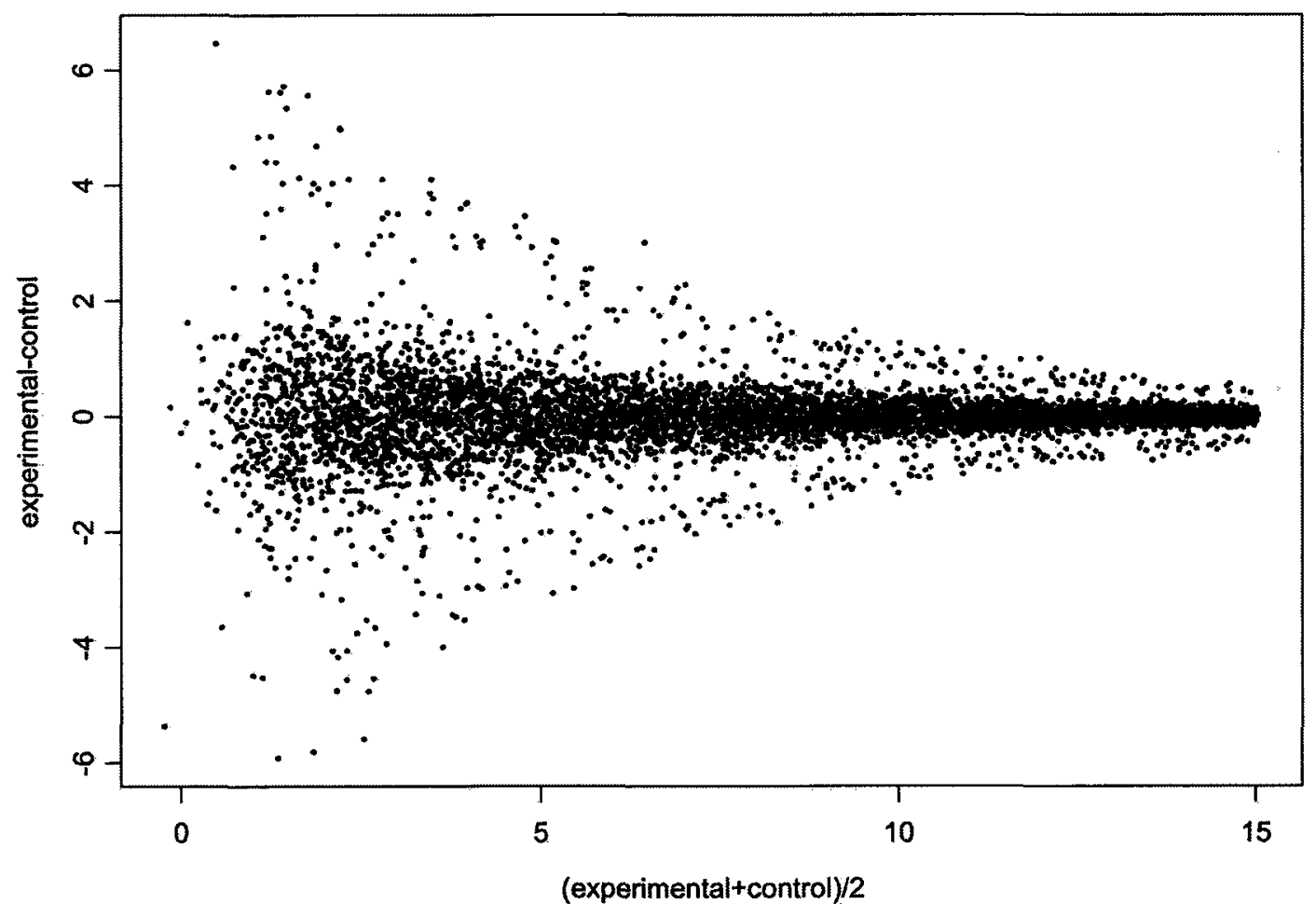

Figure 3. Differential gene expression graph. The datapoints included 0.03 normalized fraction upregulated genes, 0.94 normalized fraction neutral genes and 0.03 normalized fraction downregulated genes. 


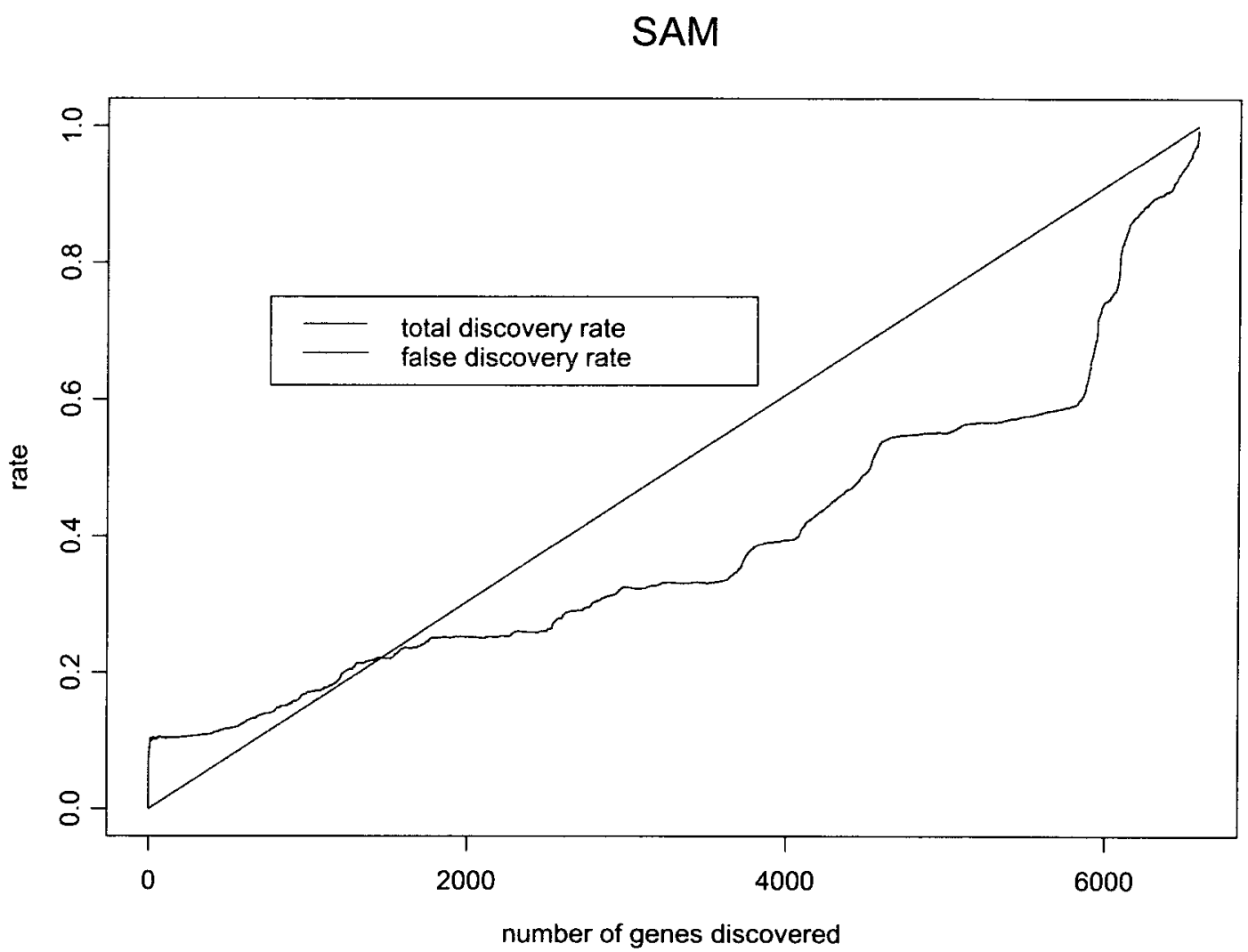

Figure 4. Significance Analysis of Microarrays (SAM) algorithm total discovery rate (TDR) graph and false discovery rate (FDR) graph. The TDR graph was represented as the green line. The FDR graph was represented as the red line. 


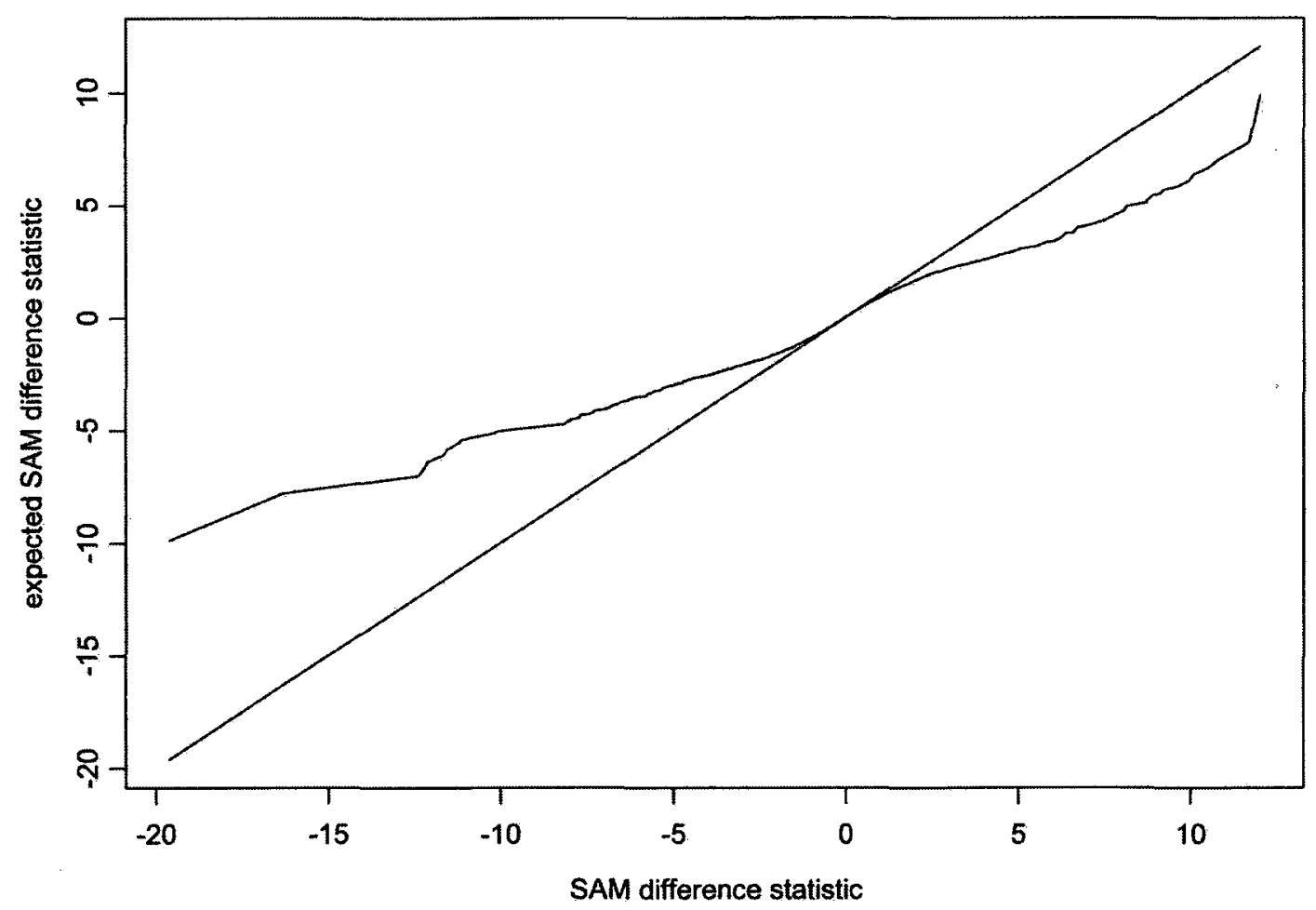

Figure 5. Significance Analysis of Microarrays (SAM) algorithm difference statistic graph and expected difference statistic graph. The nonsymmetric crossover point was represented as the noncentral intersection point between the two intersecting graphs. The nonsymmetric differentials were represented as the nonequal deviations at the extrema between the two intersecting graphs. 


\subsection{Regression analysis}

Regression analysis was defined as a mathematical technique that formulated a mathematical model of the relationship between a set of $q$ independent variables $x_{1}, x_{2}, x_{3}$ $\ldots \mathrm{x}_{\mathrm{q}}$ and a dependent variable $\mathrm{y}$. The choice of the explicit form of the model was based on such considerations as the smoothness and continuity of the dependent variable $y$ as a function of the $\mathrm{q}$ independent variables $\mathrm{x}_{1}, \mathrm{x}_{2}, \mathrm{x}_{3} \ldots \mathrm{x}_{\mathrm{q}}$. Regression models were defined to be of the general form:

$$
y=f\left(x_{1}, x_{2}, x_{3} \ldots x_{q}\right)+\varepsilon
$$

The function $\mathrm{f}$ specified the relationship between the $\mathrm{q}$ independent variables $\mathrm{x}_{1}, \mathrm{x}_{2}, \mathrm{x}_{3} \ldots$ $\mathrm{x}_{\mathrm{q}}$ and the dependent variable $\mathrm{y}$. The random error $\varepsilon$ indicated the reliance of the dependent variable $y$ on quantities other than the $q$ independent variables $x_{1}, x_{2}, x_{3} \ldots x_{q}$. Regression analysis had two objectives: 1 . formulation of a function $f$ that was a reasonable approximation of the true but unknown relationship which given the $q$ independent variables $\mathrm{x}_{1}, \mathrm{x}_{2}, \mathrm{x}_{3} \ldots \mathrm{x}_{\mathrm{q}}$ predicted the dependent variable $\mathrm{y}$ and 2 . inference about the parameters of the model.

Parametric methods were statistical procedures for testing hypotheses about the parameters in a population described by a specified distributional form. Parametric regression analysis made assumptions about the parametric form of the function $f$. Nonparametric methods were statistical techniques of estimation and inference that were based on a function of the sample observations. The probability distribution of the sample observations did not depend on a complete specification of the probability distribution of the population from which the sample was drawn. The statistical techniques were valid under general assumptions about the underlying population. Nonparametric regression analysis did not make assumptions about the parametric form of the function $\mathrm{f}$. 
Nonparametric regression analysis for estimating the function $f$ included three methods: 1. kernel regression smoothing, 2. recursive partitioning regression and 3. multivariate adaptive regression splines. Regression analysis most commonly studied models of the mean of the conditional distribution of the dependent variable $y$ given the values of the $\mathrm{q}$ independent variables $\mathrm{x}_{1}, \mathrm{x}_{2}, \mathrm{x}_{3} \ldots \mathrm{x}_{\mathrm{q}}$. The mean was the usual characteristic modeled in the conditional distribution. Modeling additional characteristics of the conditional distribution was of further interest. The conditional distribution could have been further characterized using quantiles. For $0 \leq \mathrm{p} \leq 1$, the pth conditional quantile function defined the value $\mathrm{Q}_{\mathrm{p}}\left(\mathrm{x}_{1}, \mathrm{x}_{2}, \mathrm{x}_{3} \ldots \mathrm{x}_{\mathrm{q}}\right)$ such that the probability that $\mathrm{y}<$ $\mathrm{Q}_{\mathrm{p}}\left(\mathrm{x}_{1}, \mathrm{x}_{2}, \mathrm{x}_{3} \ldots \mathrm{x}_{\mathrm{q}}\right)$ was exactly $\mathrm{p}$, conditional on the values of $\mathrm{x}_{1}, \mathrm{x}_{2}, \mathrm{x}_{3} \ldots \mathrm{x}_{\mathrm{q}}$. By choosing specific quantiles, attention was focused on additional aspects of the conditional distribution such as the upper or lower tail behaviour.

Regression analysis models were extended to estimate the quantiles of a conditional distribution based on a random sample drawn from the conditional distribution. Quantile regression models were defined to be of the general form:

$$
\mathrm{y}=\mathrm{Q}_{\mathrm{p}}\left(\mathrm{x}_{1}, \mathrm{x}_{2}, \mathrm{x}_{3} \ldots \mathrm{x}_{\mathrm{q}}\right)+\varepsilon
$$

Let $p \varepsilon(0,1)$. Each choice of $p$ focused attention on a different characteristic of the conditional distribution between the $q$ independent variables $x_{1}, x_{2}, x_{3} \ldots x_{q}$ and the dependent variable $y$. The individual conditional quantile function $Q_{p}$ specified the regression relationship between the $q$ independent variables $x_{1}, x_{2}, x_{3} \ldots x_{q}$ and the dependent variable y. Graphing a collection of conditional quantile functions visualized the entire conditional distribution. The random error $\varepsilon$ indicated the reliance of the dependent variable $y$ on quantities other than the $q$ independent variables $x_{1}, x_{2}, x_{3} \ldots x_{q}$. Quantile regression was motivated by two objectives: 1. robust estimation and 2. outlier identification.

Parametric quantile regression provided linear assumptions for quantile regression models (Koenker and Basset 1978). Nonparametric quantile regression adapted more 
flexible assumptions for complex quantile regression models. Nonparametric quantile regression only assumed that the conditional quantile distribution was a smooth function. The complexity of the quantile regression models was determined completely by the data. The underlying structure of the data revealed self expression by avoiding subjectivity in the selection of a specific parametric model. Nonparametric quantile regression used local polynomial fitting algorithms for estimating mean functions and regression quantile functions. Bootstrap estimation was a computationally-intensive method which could have been used to define confidence intervals for regression coefficients and regression quantile functions. Quantile regression has been reviewed (Koenker and Bassett 1978; Handcock and Morris 1999; Yu et al. 2003; Chen and Wei 2005; Koenker 2005; Hao and Naiman 2007).

Locally-weighted regression was defined as a method for estimating regression analysis through a smoothing procedure in which linear (degree one) and quadratic (degree two) polynomials were used to approximate the regression functions within the neighbourhood spaces of the independent variables. The local polynomial fitting of the regression functions within the neighborhood spaces of the independent variables was conducted in a moving fashion analogous to the moving average computations performed in time series analysis. Locally-weighted regression used local polynomial fitting as the estimate of the regression function without resorting to any parametric models. Locallyweighted regression fell into the category of procedures referred to as nonparametric regression analysis.

The current paradigm of regression analysis was the global fitting of parametric functions of the independent variables by least squares. Parametric classes included polynomial functions. The advantage of locally-weighted regression over the global fitting of parametric functions was that a much wider class of regression functions could have been estimated without distortion. Locally-weighted regression produced a final composite of smooth functions with each function being a linear combination of the independent variables. Locally-weighted regression was used for three purposes: 1. data 
exploration, 2. providing nonparametric regression models and 3. diagnostic verification of parametric regression models.

A robust estimation method known as the locally-weighted regression and smoothing scatterplots (LOESS or LOWESS) algorithm was used to guard against deviant point distortion of the smoothed points caused by the bias of unsymmetric data distributions. The LOWESS algorithm prevented deviant point distortion of the smoothed points using an adaptation of the iteratively reweighted least squares algorithm. The LOWESS algorithm was widely adopted in statistics as a standard for estimating smooth functions (Cleveland 1979; Cleveland and Devlin 1988; Cleveland et al. 1988; Grosse 1989).

Locally-weighted regression was a computationally-intensive method which was amenable to implementation on parallel computers (Fauntleroy and Wegman 1996). The kernel weighted local linear fitting algorithm approximated conditional quantile functions (Yu and Jones 1998). Local regression has been reviewed (Cleveland and Loader 1996; Loader 1999, 2004; Seber and Lee 2003). 


\section{Chapter 3 Differential Gene Expression (DGE) Algorithm}

The Differential Gene Expression (DGE) algorithm was presented in the detailed design specifications. The detailed design specifications formed the basis for the implementation of the DGE algorithm as computational software. The DGE algorithm was presented in five detailed design specifications: 1. strategy, 2. input data and output data, 3. normalization algorithm, 4. nonparametric quantile regression algorithm and 5. DGE algorithm logical structure.

The strategy used by the DGE algorithm was based on the nonparametric quantile regression. The nonparametric quantile regression ranks possessed two advantages: 1 . probability distribution and 2. rank stability. The nonparametric quantile regression ranks did not require the assumption of the Gaussian probability distribution. The nonparametric quantile regression ranks pooled information from all of the genes with similar expression levels to compute more stable gene rank estimates. The second advantage was particularly important under the limited conditions that existed when the gene sample sizes were small.

The input data of the Differential Gene Expression (DGE) problem consisted of a set of $n_{1}+n_{2}$ microarray datasets which were each composed of an identical number of gene transcription measurements. The gene transcription measurements were arranged in a one-to-one correspondence across all of the $n_{1}+n_{2}$ microarray datasets. The $n_{1}$ control state microarray datasets were compared to the $\mathrm{n}_{2}$ experimental state microarray datasets. The objective of the DGE problem was the categorization of the differences between the control state gene transcription measurements and the experimental state gene transcription measurements. The categorization of the differences between the control state gene transcription measurements and the experimental state gene transcription measurements resulted in three possible gene transcription classifications: 1 . positive regulation, 2. neutral regulation and 3. negative regulation. The output data of the DGE problem consisted of the entire set of gene transcription classifications for all of the corresponding gene transcription measurements. 
The novel normalization algorithm formed the preliminary step of the DGE algorithm. The normalization algorithm was the proposed method for the standardization of the signals in the control state microarray datasets and the experimental state microarray datasets to comparable levels of intensity. Transformed microarray datasets compensated for the systematic variations as a prerequisite to allowing the meaningful comparative microarray analysis between the control microarray datasets and the experimental microarray datasets in the differential gene expression algorithms.

The nonparametic quantile regression algorithm was the customized version of the precedent nonparametric quantile regression algorithm ( $\mathrm{Yu}$ and Jones 1998). The nonparametic quantile regression algorithm subjected the gene transcription measurements to a series of seventeen calculations: 1. quantile initialization algorithm, 2. median algorithm, 3. kernel localization function, 4. $S_{\mathrm{n}, \mathrm{l}}$ function, 5. weight function, 6 . matrix inversion algorithm, 7. residual squares criterion weight function, 8. residual squares criterion algorithm, $9 . \mathrm{h}_{1, \text { minimum present }}$ algorithm, 10. $\mathrm{h}_{1, \text { expansion-contraction }}$ algorithm, 11. $h_{1, \text { mean }}$ algorithm, 12. $h_{1, p}$ algorithm, 13. $h_{2, p}$ algorithm, 14. local linear kernel estimation function, 15. implicit estimation function, 16. quantile tolerance function and 17. nonparametric quantile regression algorithm.

The DGE algorithm logical structure described the structural organization of the DGE algorithm. The novel DGE algorithm was the proposed method for the solution of the DGE problem. The DGE algorithm was based on the nonparametric quantile regression algorithm.

\subsection{Differential Gene Expression (DGE) Algorithm Strategy}

The Significance Analysis of Microarrays (SAM) algorithm was based on the use of the modified T-statistic. The modified T-statistic was referred to as the difference statistic. The difference statistics were used for the statistical gene expression measurements in the SAM algorithm. The SAM algorithm made the implicit assumption 
that the observed gene expression changes were based on the Gaussian probability distribution.

The Differential Gene Expression (DGE) algorithm was based on the use of the quantile. The quantiles were used for the statistical gene expression measurements in the DGE algorithm. The DGE algorithm was based on the nonparametric quantile regression. The nonparametric quantile regression possessed two advantages: 1 . probability distribution and 2 . nonlinear function. The nonparametric quantile regression did not require the assumption of the Gaussian probability distribution. The nonparametric quantile regression did not require the assumption of the linear function between the gene expression levels and gene variance changes. The probability distribution quantiles effectively ranked the observed gene expression changes. The nonparametric quantile regression ranks computed in the DGE algorithm could have been used in the same manner as the difference statistic ranks computed in the SAM algorithm. The nonparametric quantile regression ranks possessed two advantages over the difference statistic ranks: 1. probability distribution and 2. rank stability. The nonparametric quantile regression ranks did not require the assumption of the Gaussian probability distribution. The nonparametric quantile regression ranks pooled information from all of the genes with similar expression levels to compute more stable gene rank estimates. The second advantage was particularly important under the limited conditions that existed when the gene sample sizes were small. Thus, the nonparametric quantile regression strategy extended the SAM algorithm to generate the DGE algorithm which was predicted by us to be more stable under the small gene sample size conditions.

\subsection{Input Data and Output Data}

The Differential Gene Expression (DGE) problem was a central comparative datamining research challenge in computational molecular biology. The DGE problem statement was defined by us as follows. 
Consider the general case of the DGE problem. The input data of the DGE problem consisted of a set of $n_{1}+n_{2}$ microarray datasets which were each composed of an identical number of gene transcription measurements. The gene transcription measurements were arranged in a one-to-one correspondence across all of the $n_{1}+n_{2}$ microarray datasets. The DGE problem analyzed the $n_{1}+n_{2}$ microarray datasets as an experiment consisting of two states: 1 . control state and 2. experimental state. The control state consisted of a subset of $n_{1}$ replicated microarray datasets. The experimental state consisted of a subset of $n_{2}$ replicated microarray datasets. $C\left(n_{1}+n_{2}, n_{1}\right)$ combinations were possible of the $n_{1}+n_{2}$ microarray datasets consisting of the $n_{1}$ control microarray datasets and the $\mathrm{n}_{2}$ experimental microarray datasets.

Consider the following restricted case of the DGE problem which was used for the development of three algorithms: 1. normalization algorithm, 2. Significance Analysis of Microarrays (SAM) algorithm and 3. Differential Gene Expression (DGE) algorithm. The input data of the DGE problem consisted of a set of $n_{1}+n_{2}=6$ microarray datasets which were each composed of an identical number of gene transcription measurements. The gene transcription measurements were arranged in a one-to-one correspondence across all of the $n_{1}+n_{2}=6$ microarray datasets. The DGE problem analyzed the $n_{1}+n_{2}$ $=6$ microarray datasets as an experiment consisting of two states: 1 . control state and 2 . experimental state. The control state consisted of a subset of $n_{1}=3$ replicated microarray datasets. The experimental state consisted of a subset of $n_{2}=3$ replicated microarray datasets. $C(6,3)=20$ combinations were possible of the $n_{1}+n_{2}=6$ microarray datasets consisting of the $n_{1}=3$ control microarray datasets and the $n_{2}=3$ experimental microarray datasets.

Signal intensity distributions were highly skewed about the origin. Microarray datasets could have been subjected to logarithmic transformations to reduce the skewness characteristics and achieve more symmetric signal intensity distributions. For example, given three control state microarray datasets and three experimental state microarray datasets the six microarray datasets were logarithmically-transformed and rearranged to form the Cartesian coordinates: 


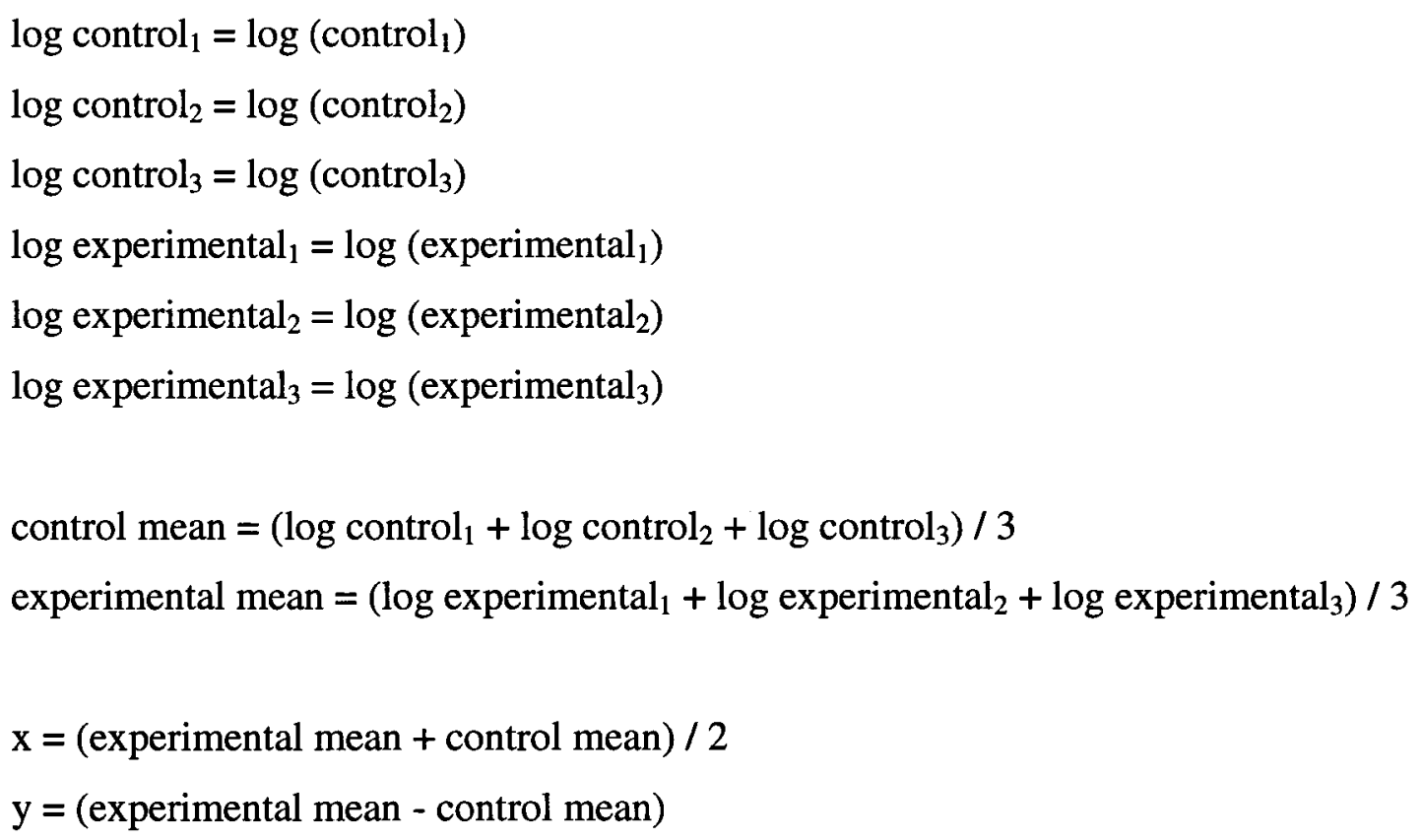

The six microarray datasets were logarithmically-transformed and rearranged to form the Cartesian coordinates in a collective process that was referred to as the data transformation. In practice, the data transformation was executed twice: 1. primary data transformation and 2. secondary data transformation. The primary data transformation was attempted on all of the input datapoints as the preliminary step to identify erroneous datapoints which were subsequently eliminated from further consideration. The secondary data transformation was repeated on the remaining datapoints as the initial step for the actual execution of three algorithms: 1. normalization algorithm, 2. SAM algorithm and 3. DGE algorithm.

The three control state microarray datasets were compared to the three experimental state microarray datasets. The objective of the DGE problem was the categorization of the differences between the control state gene transcription measurements and the experimental state gene transcription measurements. The categorization of the differences between the control state gene transcription measurements and the experimental state gene transcription measurements resulted in three possible gene transcription classifications: 1. positive regulation, 2. neutral 
regulation and 3. negative regulation. The positive regulation case signified that the control state gene transcription measurements were smaller than the experimental state gene transcription measurements. The positive regulation classification was indicative of induced gene transcription. The neutral regulation case signified that the control state gene transcription measurements were equal to the experimental state gene transcription measurements. The neutral regulation classification was indicative of unchanged gene transcription. The negative regulation case signified that the control state gene transcription measurements were larger than the experimental state gene transcription measurements. The negative regulation classification was indicative of repressed gene transcription.

The output data of the DGE problem consisted of the entire set of gene transcription classifications for all of the corresponding gene transcription measurements.

\subsection{Normalization Algorithm}

The novel normalization algorithm formed the preliminary step of the Differential Gene Expression (DGE) algorithm. The normalization algorithm was the proposed method for the standardization of the signals in the control state microarray datasets and the experimental state microarray datasets to comparable levels of intensity. Transformed microarray datasets compensated for the systematic variations as a prerequisite to allowing the meaningful comparative microarray analysis between the control microarray datasets and the experimental microarray datasets in the differential gene expression algorithms. The normalization algorithm subjected the gene transcription measurements in the three control state microarray datasets and the three experimental state microarray datasets to a series of seven calculations:

1. The six log transformations were separately computed from the six gene transcription measurements.

$\log \operatorname{control}_{1}=\log \left(\right.$ control $\left._{1}\right)$ 
$\log$ control $_{2}=\log \left(\right.$ control $\left._{2}\right)$

$\log \operatorname{control}_{3}=\log \left(\operatorname{control}_{3}\right)$

$\log$ experimental ${ }_{1}=\log \left(\right.$ experimental $\left._{1}\right)$

$\log$ experimental ${ }_{2}=\log (\text { experimental })_{2}$

$\log$ experimental ${ }_{3}=\log \left(\right.$ experimental $\left._{3}\right)$

2. The single mean was computed from the six log transformations.

mean $=\left(\log\right.$ control $_{1}+\log$ control $_{2}+\log$ control $_{3}+\log$ experimental $_{1}+\log$ experimental ${ }_{2}+\log$ experimental $\left._{3}\right) / 6$

3. The single mean was separately subtracted from the six log transformations.

$\log$ control $_{1}=\log$ control $_{1}$ - mean

$\log$ control $_{2}=\log$ control $_{2}$ - mean

$\log \mathrm{control}_{3}=\log \mathrm{control}_{3}$ - mean

$\log$ experimental $_{1}=\log$ experimental $_{1}-$ mean

$\log$ experimental ${ }_{2}=\log$ experimental ${ }_{2}$ - mean

$\log$ experimental $3=\log$ experimental $_{3}-$ mean

4. The six log transformation versus mean graphs were constructed. The log transformation versus mean graph plotted the mean on the abscissa axis and the log transformation on the ordinate axis. The six 0.50 quantiles were separately computed. The six 0.50 quantiles corresponded to the six medians.

$\operatorname{median}_{1}$

median $_{2}$

$\operatorname{median}_{3}$

median $_{4}$

medians $_{5}$

median $_{6}$ 
5. The six medians were separately subtracted from the corresponding six log transformations.

$\log$ control $_{1}=\log$ control $_{1}-$ median $_{1}$

$\log$ control $_{2}=\log$ control $_{2}-$ median $_{2}$

$\log$ control $_{3}=\log$ control $_{3}-$ median $_{3}$

$\log$ experimental $1=\log$ experimental $1-$ median $_{4}$

$\log$ experimental ${ }_{2}=\log$ experimental $2-$ median $_{5}$

$\log$ experimental ${ }_{3}=\log$ experimental $_{3}-$ median $_{6}$

6. The single mean was separately added to the six log transformations.

$\log$ control $_{1}=\log$ control $_{1}+$ mean

$\log$ control $_{2}=\log$ control $_{2}+$ mean

$\log$ control $_{3}=\log$ control $_{3}+$ mean

$\log$ experimental ${ }_{1}=\log$ experimental $_{1}+$ mean

$\log$ experimental ${ }_{2}=\log$ experimental ${ }_{2}+$ mean

$\log$ experimental ${ }_{3}=\log$ experimental ${ }_{3}+$ mean

7. The six normalized gene transcription measurements were separately computed as antilogs from the six log transformations.

$$
\begin{aligned}
& \operatorname{control}_{1}=\mathrm{e}^{(\log \text { control })} \\
& \text { control } \left._{2}=\mathrm{e}^{(\log \text { control }}{ }_{2}\right) \\
& \text { control } \left._{3}=\mathrm{e}^{(\log \text { control }}\right)_{3} \\
& \text { experimental } \left._{1}=\mathrm{e}^{(\log \text { experimental }}{ }_{1}\right) \\
& \text { experimental }{ }_{2}=\mathrm{e}^{(\text {log experimental })} \\
& \text { experimental } \left._{3}=\mathrm{e}^{(\text {log experimental }}{ }_{3}\right)
\end{aligned}
$$

\subsection{Nonparametric Quantile Regression Algorithm}


The nonparametric quantile regression algorithm used the normalized microarray datasets to compute the ninety nine quantiles. The nonparametric quantile regression algorithm formed the most complex step of the Differential Gene Expression (DGE) algorithm. The nonparametic quantile regression algorithm was the customized version of the precedent nonparametric quantile regression algorithm (Yu and Jones 1998). The nonparametic quantile regression algorithm differed from the precedent nonparametric quantile regression algorithm in the revised method for the $h_{1, \text { mean }}$ bandwidth computation. The nonparametic quantile regression algorithm subjected the gene transcription measurements to a series of seventeen calculations: 1. quantile initialization algorithm, 2. median algorithm, 3. kernel localization function, 4. $\mathrm{S}_{\mathrm{n}, 1}$ function, 5. weight function, 6. matrix inversion algorithm, 7. residual squares criterion weight function, 8 . residual squares criterion algorithm, $9 . h_{1, \text { minimum present }}$ algorithm, $10 . h_{1 \text {,expansion-contraction }}$ algorithm, 11. $h_{1, \text { mean }}$ algorithm, 12. $h_{1, p}$ algorithm, 13. $h_{2, p}$ algorithm, 14. local linear kernel estimation function, 15. implicit estimation function, 16. quantile tolerance function and 17. nonparametric quantile regression algorithm. The logical structure for the procedural invocation of the nonparametric quantile regression algorithm was defined using the call tree (Figure 6). The individual calculations are subsequently described in further detail. 
nonparametric quantile regression algorithm

input

quantile initialization algorithm

$\mathrm{h}_{1, \text { mean }}$ algorithm

$\mathrm{h}_{1, \text { minimum present }}$ algorithm

residual squares criterion algorithm

median algorithm

residual squares criterion weight function

matrix inversion algorithm

$\mathrm{h}_{1 \text {,expansion-contraction algorithm }}$

residual squares criterion algorithm

median algorithm

residual squares criterion weight function

$\mathrm{h}_{1, \mathrm{p}}$ algorithm

matrix inversion algorithm

standard normal density function

standard normal distribution function

$\mathrm{h}_{2, \mathrm{p}}$ algorithm

implicit estimation function

local linear kernel estimation function

weight function

kernel localization function

$\mathrm{S}_{\mathrm{n}, \mathrm{I}}$ function

weight function

kernel localization function

kernel localization function

$S_{n, 1}$ function

kernel localization function

identity function

quantile tolerance function

output

Figure 6. Nonparametric quantile regression algorithm call tree. 


\subsubsection{Quantile Initialization Algorithm}

The quantile initialization algorithm computed the initial values of the quantiles. The quantile initialization algorithm subjected the gene transcription measurements to a series of eleven calculations:

1. The input consisted of the set of $x_{1}, x_{2}, x_{3}, \ldots x_{n}$ datapoints and the set of $y_{1}, y_{2}, y_{3}, \ldots$ $\mathrm{y}_{\mathrm{n}}$ datapoints which were coordinately joined to form the set of ordered pairs: $\left(\mathrm{x}_{1}, \mathrm{y}_{1}\right),\left(\mathrm{x}_{2}\right.$, $\left.y_{2}\right),\left(x_{3}, y_{3}\right), \ldots\left(x_{n}, y_{n}\right)$. The input also consisted of the ten $x$ grid points: $x_{\text {grid, } 1}, x_{\text {grid,2, }}$ $\mathrm{x}_{\text {grid }, 3}, \ldots \mathrm{x}_{\text {grid, } 10}$.

2. The $\left(x_{1}, y_{1}\right),\left(x_{2}, y_{2}\right),\left(x_{3}, y_{3}\right), \ldots\left(x_{n}, y_{n}\right)$ ordered pairs were sorted into ascending $x$ order using the $\mathrm{x}_{1}, \mathrm{x}_{2}, \mathrm{x}_{3}, \ldots \mathrm{x}_{\mathrm{n}}$ datapoints as the primary key.

3. The ascending sorted $\left(x_{1}, y_{1}\right),\left(x_{2}, y_{2}\right),\left(x_{3}, y_{3}\right), \ldots\left(x_{n}, y_{n}\right)$ ordered pairs were partitioned into separate consecutive blocks of ninety nine ordered pairs.

4. Each block consisted of ninety nine ordered pairs: $\left(\mathrm{x}_{1}, \mathrm{y}_{1}\right),\left(\mathrm{x}_{2}, \mathrm{y}_{2}\right),\left(\mathrm{x}_{3}, \mathrm{y}_{3}\right), \ldots\left(\mathrm{x}_{99}\right.$, $\mathrm{y}_{99}$ ). The $\mathrm{y}_{1}, \mathrm{y}_{2}, \mathrm{y}_{3}, \ldots \mathrm{y}_{99}$ datapoints were copied and independently resorted into ascending y order.

5. Each block used linear regression to establish the interpolation mapping function from the 1 - 99 percentile independent variable onto the ascending sorted $y_{1}, y_{2}, y_{3}, \ldots y_{99}$ datapoints dependent variable.

6. The ascending sorted $x_{1}, x_{2}, x_{3}, \ldots x_{n}$ datapoints from step 2 were evenly divided into

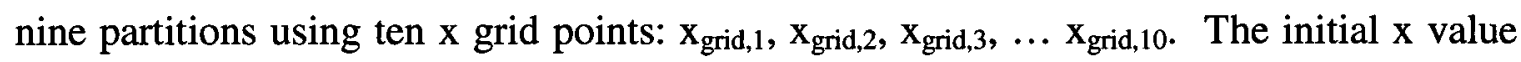

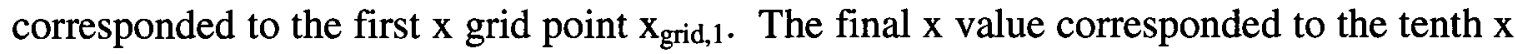

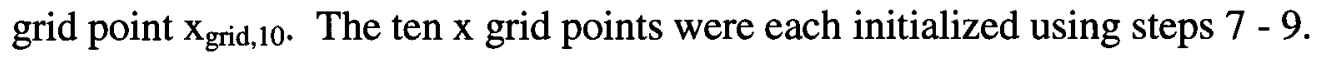

7. Each $x$ grid point $x_{\text {grid,i }}$ on the independent $x$ variable was initialized with ninety nine

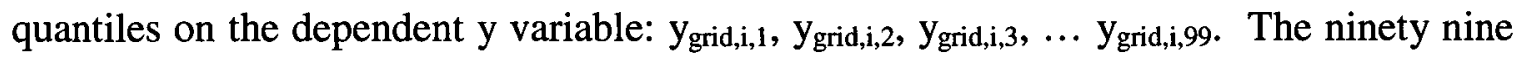
quantiles were each initialized using steps 8 - 9. The ninety nine quantiles corresponded to the ninety nine $y$ grid points.

8. Each $x$ grid point $x_{\text {grid, } i}$ was superimposed onto the ascending sorted $x_{1}, x_{2}, x_{3}, \ldots x_{n}$ datapoints from step 2. The appropriate partitioned block was determined. 
9. Each $x$ grid point $x_{\text {grid, }}$ was superimposed onto the appropriate partitioned block. The partitioned block had its own individual linear regression as the mapping function to compute the translation from the 1 - 99 percentile independent variable onto the $y_{1}, y_{2}, y_{3}$,

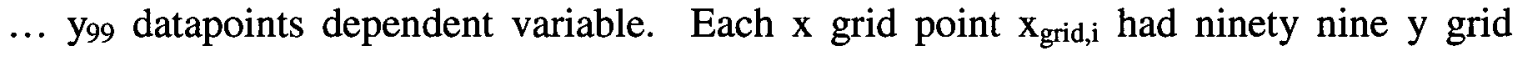
points: $y_{\text {grid,i,1, }} y_{\text {grid,i,2, }} y_{\text {grid,i,3, }}, y_{\text {grid,i,99. }}$

10. The ten $x$ grid points $x_{\text {grid,1 }}, x_{\text {grid,2 }}, x_{\text {grid,3 }}, \ldots x_{\text {grid,10 }}$ each corresponded to ninety nine $y$ grid points $y_{\text {grid,i,1, }} y_{\text {grid,i,2, }} y_{\text {grid,i,3, }}, y_{\text {grid,i,99 }}$ which formed the total of nine hundred

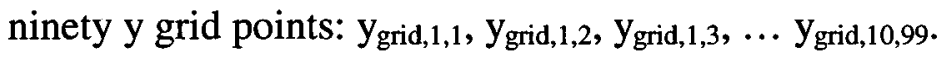

11. Output the nine hundred ninety y grid points.

$\mathrm{n}=$ number of datapoint set elements.

$\mathrm{x}_{1}, \mathrm{x}_{2}, \mathrm{x}_{3}, \ldots \mathrm{x}_{\mathrm{n}}=\mathrm{x}$ datapoint set of $\mathrm{n}$ elements.

$\mathrm{x}_{\text {grid,1, }}, \mathrm{x}_{\text {grid,2 }}, \mathrm{x}_{\text {grid,3 }}, \ldots \mathrm{x}_{\text {grid,10 }}=\mathrm{x}$ gridpoint set of 10 elements.

$\mathrm{y}_{1}, \mathrm{y}_{2}, \mathrm{x}_{3}, \ldots \mathrm{y}_{\mathrm{n}}=\mathrm{y}$ datapoint set of $\mathrm{n}$ elements.

$\mathrm{y}_{\text {grid,i,1 }}, \mathrm{y}_{\text {grid,i,2, }} \mathrm{y}_{\text {grid,i,3, }}, \ldots \mathrm{y}_{\text {grid,i,99 }}=\mathrm{y}$ gridpoint set of 99 elements corresponding to the $\mathrm{x}$ gridpoint $\mathrm{i}$.

$y_{\text {grid,1,1 }}, y_{\text {grid,1,2, }} y_{\text {grid,1,3 }}, \ldots y_{\text {grid,10,99 }}=y$ gridpoint set of 990 elements.

$\left(x_{1}, y_{1}\right),\left(x_{2}, y_{2}\right),\left(x_{3}, y_{3}\right), \ldots\left(x_{n}, y_{n}\right)=(x, y)$ datapoint set of $n$ ordered pairs.

\subsubsection{Median Algorithm}

The median algorithm computed the median $\mathrm{x}_{0}$ of the $\mathrm{x}_{\mathrm{n}}$ datapoints.

\subsubsection{Kernel Localization Function}

The kernel localization function $K$ was the symmetric probability density function. The kernel localization function $\mathrm{K}$ was the local weighting function that assigned high weights to the values which were close to the argument $\mathrm{z}$. The kernel localization function $\mathrm{K}$ was defined:

$\mathrm{K}(\mathrm{z} / \mathrm{h})=(1 /(\sqrt{ }(2 * \pi))) *\left(\operatorname{exponent}\left(\left(-\left(\mathrm{z}^{2}\right)\right) /\left(2 * \mathrm{~h}^{2}\right)\right)\right)$ 
$\mathrm{h}=$ bandwidth which was the scale parameter that controlled the amount of smoothing applied to the regression data.

$\mathrm{K}=$ symmetric probability density function value.

$\mathrm{z}=$ input variable.

\subsection{4 $\mathrm{S}_{\mathrm{n}, \mathrm{l}}$ Function}

The $S_{n, 1}$ function was the weighted sum of the kernel localization functions that was used to define the weight function. The $S_{n, l}$ function was defined:

$\mathrm{S}_{\mathrm{n}, \mathrm{l}}=\left(\Sigma_{\mathrm{i}}\left(\mathrm{K}\left(\left(\mathrm{x}_{\text {grid }}-\mathrm{x}_{\text {data, }}\right) / \mathrm{h}_{1}\right)\right) *\left(\mathrm{x}_{\text {grid }}-\mathrm{x}_{\text {data, }, \mathrm{i}}\right)^{\mathbf{l}}\right)$

$\mathrm{h}_{1}=$ bandwidth which was the scale parameter that controlled the amount of $\mathrm{x}$ direction smoothing applied to the regression data.

$\mathrm{K}=$ kernel localization function.

$1=1$ or 2 .

$\mathrm{S}_{\mathrm{n}, \mathrm{l}}=\mathrm{S}_{\mathrm{n}, \mathrm{l}}$ function value.

$\mathrm{x}_{\text {data, } \mathrm{i}}=\mathrm{x}$ datapoint $\mathrm{i}$.

$\mathrm{x}_{\text {data } \mathrm{j}}=\mathrm{x}$ datapoint $\mathrm{j}$.

$\mathrm{x}_{\text {grid }}=\mathrm{x}$ gridpoint.

\subsubsection{Weight Function}

The weight function $w$ was associated with the local linear fitting. The weight function $w$ was used to construct the matrix $S$ and the matrix $S^{*}$ that formed the core of the residual squares criterion (RSC) algorithm. The weight function w was defined:

$\mathrm{w}_{\mathrm{j}}\left(\mathrm{x}_{\text {grid }}\right)=\left(\left(\mathrm{K}\left(\left(\mathrm{x}_{\text {grid }}-\mathrm{x}_{\text {data,j }}\right) / \mathrm{h}_{1}\right)\right) *\left(\mathrm{~S}_{\mathrm{n}, 2}-\left(\left(\mathrm{x}_{\text {grid }}-\mathrm{x}_{\text {data, } \mathrm{j}}\right) * \mathrm{~S}_{\mathrm{n}, 1}\right)\right)\right)$ 
$\mathrm{h}_{1}=$ bandwidth which was the scale parameter that controlled the amount of $\mathrm{x}$ direction smoothing applied to the regression data.

$\mathrm{K}=$ kernel localization function.

$\mathrm{S}_{\mathrm{n}, 1}=\mathrm{S}_{\mathrm{n}, 1}$ function.

$\mathrm{S}_{\mathrm{n}, 2}=\mathrm{S}_{\mathrm{n}, 2}$ function.

$\mathrm{w}=$ weight between the $\mathrm{x}$ gridpoint and the $\mathrm{x}$ datapoint $\mathrm{j}$.

$\mathrm{x}_{\mathrm{data}, \mathrm{j}}=\mathrm{x}$ datapoint $\mathrm{j}$.

$\mathrm{x}_{\text {grid }}=\mathrm{x}$ gridpoint.

\subsubsection{Matrix Inversion Algorithm}

The matrix inversion algorithm computed the inverse $S^{-1}$ of the $2 \times 2$ square matrix S.

\subsubsection{Residual Squares Criterion Weight Function}

The residual squares criterion (RSC) weight function computed the RSC weight between two datapoints. The RSC weight function was defined:

weight $=\left(\left(\mathrm{e}^{* *}\left(\left(-1 *\left(\left(\mathrm{x}_{\mathrm{i}}-\mathrm{x}_{\mathrm{j}}\right)^{2}\right)\right) /\left(2 *\left(\mathrm{~h}_{1}\right)^{2}\right)\right)\right) /(\sqrt{ }(2 * \pi))\right)$

$\mathrm{h}_{1}=$ bandwidth which was the scale parameter that controlled the amount of $\mathrm{x}$ direction smoothing applied to the regression data.

weight $=$ residual squares criterion weight between the $x$ datapoint $\mathrm{i}$ and the $\mathrm{x}$ datapoint $\mathrm{j}$.

$\mathrm{x}_{\mathrm{i}}=\mathrm{x}$ datapoint $\mathrm{i}$.

$\mathrm{x}_{\mathbf{j}}=\mathrm{x}$ datapoint $\mathrm{j}$.

\subsubsection{Residual Squares Criterion Algorithm}


The residual squares criterion (RSC) algorithm computed the error estimate of the $\mathrm{h}$ bandwidth. The RSC algorithm subjected the gene transcription measurements to a series of eighteen calculations:

1. The input consisted of the $h_{1}$ bandwidth and the set of $x_{1}, x_{2}, x_{3}, \ldots x_{n}$ datapoints.

2. $\mathrm{x}_{0}=\operatorname{median}\left(\mathrm{x}_{1}, \mathrm{x}_{2}, \mathrm{x}_{3}, \ldots \mathrm{x}_{\mathrm{n}}\right)$.

3. For $i=1$ to $n$

Begin

$$
\mathrm{w}_{\mathrm{i}, 0}=\text { residual squares criterion weight }\left(\mathrm{x}_{\mathrm{i}}, \mathrm{x}_{0}, \mathrm{~h}_{1}\right) \text {. }
$$

End.

4. matrix $S=\left[\Sigma_{\mathrm{i}} \mathrm{w}_{\mathrm{i}, 0}\right.$ $\left.\Sigma_{\mathrm{i}} \mathrm{w}_{\mathrm{i}, 0}\left(\mathrm{x}_{\mathrm{i}}-\mathrm{x}_{0}\right)\right]$ $\left[\Sigma_{\mathrm{i}} \mathbf{w}_{\mathrm{i}, 0}\left(\mathbf{x}_{\mathrm{i}}-\mathrm{x}_{0}\right)\right.$ $\left.\Sigma_{\mathrm{i}} \mathrm{w}_{\mathrm{i}, 0}\left(\mathrm{x}_{\mathrm{i}}-\mathrm{x}_{0}\right)^{2}\right]$

5. matrix $\mathrm{S}^{*}=\left[\Sigma_{\mathrm{i}} \mathrm{w}_{\mathrm{i}, 0}{ }^{2}\right.$ $\left.\Sigma_{\mathrm{i}} \mathrm{w}_{\mathrm{i}, 0}{ }^{2}\left(\mathrm{x}_{\mathrm{i}}-\mathrm{x}_{0}\right)\right]$ $\left[\Sigma_{\mathrm{i}} \mathrm{w}_{\mathrm{i}, 0}{ }^{2}\left(\mathrm{x}_{\mathbf{i}}-\mathrm{x}_{0}\right)\right.$ $\left.\Sigma_{\mathrm{i}} \mathrm{w}_{\mathrm{i}, 0}{ }^{2}\left(\mathrm{x}_{\mathrm{i}}-\mathrm{x}_{0}\right)^{2}\right]$

6. matrix $S^{-1}=$ matrix inversion (S).

7. matrix $B=\left(S^{-1} * S^{*}\right)$.

8. trace $=\left(B_{1,1}+B_{2,2}\right)$.

9. $\mathrm{T}=\left(\Sigma_{\mathrm{i}} \mathrm{w}_{\mathrm{i}, 0}-\right.$ trace $)$.

10. matrix $A=\left(S^{-1} * S^{*} * S^{-1}\right)$.

11. $\mathrm{V}=\mathrm{A}_{1,1}$.

12. The evenly-distributed subset of $x_{1}, x_{2}, x_{3}, \ldots x_{m}$ datapoints was selected from the original set of $x_{1}, x_{2}, x_{3}, \ldots x_{n}$ datapoints. The subset of datapoints consisted of $m=99$ elements.

13. The evenly-distributed subset of $y_{1}, y_{2}, y_{3}, \ldots y_{m}$ datapoints was selected from the original set of $y_{1}, y_{2}, y_{3}, \ldots y_{n}$ datapoints. The subset of datapoints consisted of $m=99$ elements.

14. For $\mathrm{i}=1$ to $\mathrm{m}$

Begin

$$
\begin{array}{r}
\text { For } \mathrm{j}=1 \text { to } \mathrm{n} \\
\text { Begin }
\end{array}
$$

15. $W_{i, j}=$ residual squares criterion weight $\left(x_{i}, x_{j}, h_{1}\right)$. 


\section{End}

16. $\mathrm{y}_{\text {hat }, \mathrm{i}}=\left(\left(\Sigma_{\mathrm{j}}\left(\mathrm{W}_{\mathrm{i}, \mathrm{j}} * \mathrm{y}_{\mathrm{j}}\right)\right) /\left(\Sigma_{\mathrm{j}} \mathrm{W}_{\mathrm{i}, \mathrm{j}}\right)\right)$.

End.

17. $\mathrm{RSC}=\left(\left(\Sigma_{\mathrm{i}}\left(\mathrm{y}_{\mathrm{i}}-\mathrm{y}_{\mathrm{hat}, \mathrm{i}}\right)^{2}\right) *((1+(2 * \mathrm{~V})) / \mathrm{T})\right)$.

18. Output the RSC.

$\mathrm{A}=$ two element by two element square matrix.

$A_{1,1}=$ first diagonal element of the A matrix.

$B=$ two element by two element square matrix.

$\mathrm{h}_{1}=$ bandwidth which was the scale parameter that controlled the amount of $\mathrm{x}$ direction smoothing applied to the regression data.

$\mathrm{m}=$ number of datapoint subset elements.

$\mathrm{n}=$ number of datapoint set elements.

$\mathrm{RSC}=$ residual squares criterion error estimate of the $\mathrm{h}$ bandwidth.

$\mathrm{S}=$ two element by two element square matrix.

$S^{*}=$ two element by two element square matrix.

$\mathrm{S}^{-1}=$ two element by two element square matrix.

$\mathrm{T}=$ difference between the cumulative sum of the weight function estimates at the median and the trace.

trace $=$ sum of the diagonal elements of the A matrix.

$V=$ first diagonal element $A_{1,1}$ of the A matrix.

$\mathrm{w}_{\mathrm{i}, 0}=$ weight function estimate at the median.

$\mathrm{W}_{\mathrm{i}, \mathrm{j}}=$ residual squares criterion weight function estimate between the $\mathrm{x}$ datapoint $\mathrm{i}$ and the $\mathrm{x}$ datapoint $\mathrm{j}$.

$\mathrm{w}_{\mathrm{i}}\left(\mathrm{x}_{0}\right)=$ weight function estimate at the median.

$\mathrm{x}_{\mathbf{i}}=\mathrm{x}$ datapoint $\mathrm{i}$.

$\mathrm{x}_{\mathrm{j}}=\mathrm{x}$ datapoint $\mathrm{j}$.

$\mathrm{x}_{0}=\mathrm{x}$ median.

$\mathrm{x}_{1}, \mathrm{x}_{2}, \mathrm{x}_{3}, \ldots \mathrm{x}_{\mathrm{m}}=\mathrm{x}$ datapoint subset of $\mathrm{m}$ elements.

$\mathrm{x}_{1}, \mathrm{x}_{2}, \mathrm{x}_{3}, \ldots \mathrm{x}_{\mathrm{n}}=\mathrm{x}$ datapoint set of $\mathrm{n}$ elements.

$\mathrm{y}_{\text {hat }, \mathrm{i}}=\mathrm{y}$ datapoint estimate $\mathrm{i}$. 
$y_{i}=y$ datapoint $i$.

$\mathrm{y}_{\mathrm{j}}=\mathrm{y}$ datapoint $\mathrm{j}$.

$y_{1}, y_{2}, y_{3}, \ldots y_{m}=y$ datapoint subset of $m$ elements.

$y_{1}, y_{2}, y_{3}, \ldots y_{n}=y$ datapoint set of $n$ elements.

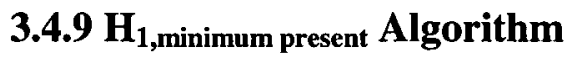

The $\mathrm{h}_{1 \text {,minimum present }}$ algorithm determined whether the minimum residual squares criterion (RSC) error estimate existed in the RSC versus bandwidth curve. The presence of the minimum RSC error estimate indicated that the RSC versus bandwidth curve was nonmonotonic. The absence of the minimum RSC error estimate indicated that the RSC

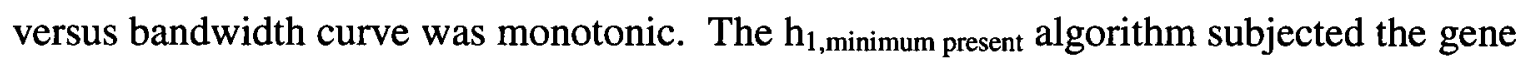
transcription measurements to a series of seventeen calculations:

1. The input consisted of the set of $x_{1}, x_{2}, x_{3}, \ldots x_{n}$ datapoints.

Comment: steps 2 - 3 defined the $h$ search space.

2. $h_{\text {initial }}=\left(\left(x_{\text {maximum }}-x_{\text {minimum }}\right) / 10\right)$.

3. $\mathrm{x}_{\text {range }}=\left(\mathrm{x}_{\text {maximum }}-\mathrm{x}_{\text {minimum }}\right)$.

Comment: steps 4 - 12 computed the successive rightward extensions of the $h$ bandwidth until reaching the termination condition within two successive iterations: $\left(\left(\mathrm{RSC}_{\mathrm{old}} \leq\right.\right.$ $\left.\operatorname{RSC}_{\text {new }}\right)$ or $\left.\left(\mathrm{h}_{\text {new }} \geq \mathrm{x}_{\text {range }}\right)\right)$.

4. $\mathrm{h}_{\mathrm{old}}=\mathrm{h}_{\text {initial }}$.

5. $\mathrm{RSC}_{\mathrm{old}}=\mathrm{RSC}\left(\mathrm{h}_{\mathrm{old}}\right)$.

6. $\mathrm{h}_{\text {new }}=\left(2 * \mathrm{~h}_{\text {old }}\right)$.

7. $\operatorname{RSC}_{\text {new }}=\operatorname{RSC}\left(\mathrm{h}_{\text {new }}\right)$.

8. While ( $\left(\mathrm{RSC}_{\text {old }}>\mathrm{RSC}_{\text {new }}\right)$ and $\left.\left(\mathrm{h}_{\text {new }}<\mathrm{x}_{\text {range }}\right)\right)$

Begin

9. $\mathrm{h}_{\text {old }}=\mathrm{h}_{\text {new }}$.

10. $\mathrm{RSC}_{\text {old }}=\mathrm{RSC}_{\text {new }}$.

11. $\mathrm{h}_{\text {new }}=\left(2 * \mathrm{~h}_{\text {old }}\right)$.

12. $\mathrm{RSC}_{\text {new }}=\operatorname{RSC}\left(\mathrm{h}_{\text {new }}\right)$. 
End

Comment: steps 13 - 17 computed the post-extension analysis that determined which of the two termination conditions stopped the rightward extensions of the $h$ bandwidth.

13. If $\left(\left(\mathrm{RSC}_{\text {old }}>\mathrm{RSC}_{\text {new }}\right)\right.$ and $\left.\left(\mathrm{h}_{\text {new }} \geq \mathrm{x}_{\text {range }}\right)\right)$

Then

Begin

14. minimum_present $=$ false.

End

15. If $\left(\mathrm{RSC}_{\text {old }} \leq \mathrm{RSC}_{\text {new }}\right)$

Then

Begin

16. minimum_present $=$ true.

End

17. Output the minimum_present.

$\mathrm{h}_{\text {initial }}=$ initial $\mathrm{h}$ bandwidth .

$\mathrm{h}_{\text {new }}=$ next estimate of the $\mathrm{h}$ bandwidth.

$\mathrm{h}_{\mathrm{old}}=$ previous estimate of the $\mathrm{h}$ bandwidth.

minimum_present $=$ Boolean variable which indicated whether the minimum residual squares criterion error estimate existed in the residual squares criterion versus bandwidth curve.

$\mathrm{n}=$ number of datapoint set elements.

$\mathrm{RSC}_{\text {old }}=$ residual squares criterion error estimate of the $\mathrm{h}_{\mathrm{old}}$ bandwidth.

$\mathrm{RSC}_{\text {new }}=$ residual squares criterion error estimate of the $\mathrm{h}_{\text {new }}$ bandwidth.

$\mathbf{x}_{\text {maximum }}=\mathrm{x}$ maximum datapoint.

$\mathrm{x}_{\text {minimum }}=\mathrm{x}$ minimum datapoint.

$\mathrm{x}_{\text {range }}=$ difference between the $\mathrm{x}$ maximum datapoint and the $\mathrm{x}$ minimum datapoint .

$\mathrm{x}_{1}, \mathrm{x}_{2}, \mathrm{x}_{3}, \ldots \mathrm{x}_{\mathrm{n}}=\mathrm{x}$ datapoint set of $\mathrm{n}$ elements.

\subsubsection{0 $\mathrm{H}_{1}$,expansion-contraction Algorithm}




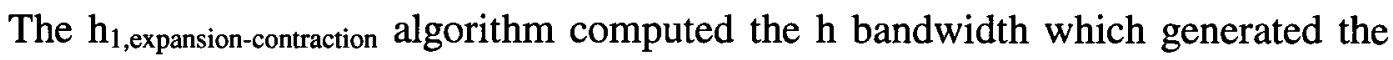
minimum residual squares criterion (RSC) error estimate in the RSC versus bandwidth

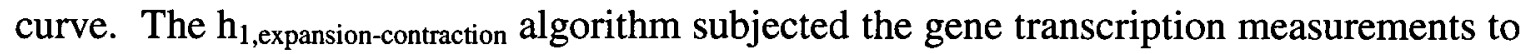
a series of fifty seven calculations:

1. The input consisted of the set of $x_{1}, x_{2}, x_{3}, \ldots x_{n}$ datapoints.

Comment: steps 2 - 3 defined the h search space.

2. $\mathrm{h}_{\text {initial }}=\left(\left(\mathrm{x}_{\text {maximum }}-\mathrm{x}_{\text {minimum }}\right) / 10\right)$.

3. $\mathrm{x}_{\mathrm{range}}=\left(\mathrm{x}_{\text {maximum }}-\mathrm{x}_{\text {minimum }}\right)$.

Comment: steps 4 - 12 computed the successive leftward extensions of the $\mathrm{h}$ bandwidth until reaching the termination condition within two successive iterations: $\left(\mathrm{RSC}_{\text {new }}>\right.$ $\mathrm{RSC}_{\text {old }}$ ). Steps 4 - 12 defined the left boundary of the h search space.

4. $\mathrm{h}_{\text {old }}=\mathrm{h}_{\text {initial }}$.

5. $\mathrm{RSC}_{\mathrm{old}}=\mathrm{RSC}\left(\mathrm{h}_{\mathrm{old}}\right)$.

6. $\mathrm{h}_{\text {new }}=\left(0.5 * \mathrm{~h}_{\text {old }}\right)$.

7. $\mathrm{RSC}_{\text {new }}=\mathrm{RSC}\left(\mathrm{h}_{\text {new }}\right)$.

8. While $\left(\mathrm{RSC}_{\text {new }} \leq \mathrm{RSC}_{\text {old }}\right)$

Begin

9. $\mathrm{h}_{\text {old }}=\mathrm{h}_{\text {new }}$.

10. $\mathrm{RSC}_{\text {old }}=\mathrm{RSC}_{\text {new }}$.

11. $\mathrm{h}_{\text {new }}=\left(0.5 * \mathrm{~h}_{\text {old }}\right)$.

12. $\operatorname{RSC}_{\text {new }}=\operatorname{RSC}\left(\mathrm{h}_{\text {new }}\right)$.

End

Comment: steps 13 - 14 saved the left boundary of the h search space.

13. $\mathrm{h}_{\text {left }}=\mathrm{h}_{\text {new }}$.

14. $h_{\text {saved left }}=h_{\text {new }}$.

Comment: steps 15 - 23 computed the successive rightward extensions of the $h$ bandwidth until reaching the termination condition within two successive iterations: $\left(\left(\mathrm{RSC}_{\text {old }} \leq \mathrm{RSC}_{\text {new }}\right)\right.$ or $\left.\left(\mathrm{h}_{\text {new }} \geq \mathrm{x}_{\text {range }}\right)\right)$. Steps $15-23$ defined the right boundary of the $\mathrm{h}$ search space. 
15. $\mathrm{h}_{\text {old }}=\mathrm{h}_{\text {initiai }}$.

16. $\mathrm{RSC}_{\mathrm{old}}=\mathrm{RSC}\left(\mathrm{h}_{\mathrm{old}}\right)$.

17. $\mathrm{h}_{\text {new }}=\left(2 * \mathrm{~h}_{\text {old }}\right)$.

18. $\mathrm{RSC}_{\text {new }}=\operatorname{RSC}\left(\mathrm{h}_{\text {new }}\right)$.

19. While ( $\left(\mathrm{RSC}_{\text {old }}>\mathrm{RSC}_{\text {new }}\right)$ and $\left.\left(\mathrm{h}_{\text {new }}<\mathrm{x}_{\text {range }}\right)\right)$

Begin

20. $h_{\text {old }}=h_{\text {new }}$.

21. $\mathrm{RSC}_{\text {old }}=\mathrm{RSC}_{\text {new }}$.

22. $\mathrm{h}_{\text {new }}=\left(2 * \mathrm{~h}_{\text {old }}\right)$.

23. $\operatorname{RSC}_{\text {new }}=\operatorname{RSC}\left(\mathrm{h}_{\text {new }}\right)$.

End

Comment: steps 24 - 28 computed the post-extension analysis that determined which of the two termination conditions stopped the rightward extensions of the $\mathrm{h}$ bandwidth.

24. If $\left(\left(\operatorname{RSC}_{\text {old }}>\mathrm{RSC}_{\text {new }}\right)\right.$ and $\left.\left(\mathrm{h}_{\text {new }} \geq \mathrm{x}_{\text {range }}\right)\right)$

Then

Begin

25. output error.

End

26. If $\left(\mathrm{RSC}_{\text {old }} \leq \mathrm{RSC}_{\text {new }}\right)$

Then

Begin

Comment: steps 27 - 28 saved the right boundary of the $h$ search space.

27. $\mathrm{h}_{\text {right }}=\mathrm{h}_{\text {new }}$.

28. $\mathrm{h}_{\text {saved right }}=\mathrm{h}_{\text {new }}$.

End

Comment: steps 29 - 45 computed the $\mathrm{h}$ bandwidth which generated the minimum residual squares criterion error estimate in the residual squares criterion versus bandwidth curve. The left boundary and the right boundary underwent successive contractions until the two boundaries approximated the $\mathrm{h}$ bandwidth which generated the minimum residual 
squares criterion error estimate when $\left(\left(h_{\text {right }}-h_{\text {left }}\right) \leq\right.$ tolerance $)$. Steps $29-36$ computed the tolerance.

29. tolerance $_{\text {absolute }}=(0.01)$.

30. tolerance $_{\text {relative }}=\left(0.01 *\left(\mathrm{~h}_{\text {saved right }}-\mathrm{h}_{\text {saved left }}\right)\right)$.

31 . tolerance $e_{\text {minimum }}=(0.000000000000001)$.

32. tolerance $=$ tolerance $_{\text {minimum }}$.

33. If ((tolerance minimum $<$ tolerance $\left._{\text {absolute }}\right)$ and $\left(\right.$ tolerance $_{\text {absolute }} \leq$ tolerance $\left._{\text {relative }}\right)$ )

Then

Begin

34. tolerance $=$ tolerance $_{\text {absolute }}$.

End

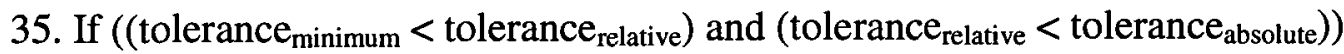

Then

Begin

36. tolerance $=$ tolerance $_{\text {relative }}$.

End

37. While $\left(\left(h_{\text {right }}-h_{\text {left }}\right)>\right.$ tolerance $)$

Begin

Comment: steps 38 - 45 compared the $1 / 3$ intermediate point and the $2 / 3$ intermediate point.

38. $\mathrm{h}_{1 / 3}=\left(\mathrm{h}_{\text {left }}+\left((1 / 3) *\left(\mathrm{~h}_{\text {right }}-\mathrm{h}_{\text {left }}\right)\right)\right)$.

39. $\mathrm{RSC}_{1 / 3}=\operatorname{RSC}\left(\mathrm{h}_{1 / 3}\right)$.

40. $\mathrm{h}_{2 / 3}=\left(\mathrm{h}_{\text {left }}+\left((2 / 3) *\left(\mathrm{~h}_{\text {right }}-\mathrm{h}_{\text {left }}\right)\right)\right)$.

41. $\mathrm{RSC}_{2 / 3}=\operatorname{RSC}\left(\mathrm{h}_{2 / 3}\right)$.

42. If $\left(\mathrm{RSC}_{1 / 3} \leq \mathrm{RSC}_{2 / 3}\right)$

Then

Begin

Comment: step 43 moved the right boundary to the

left.

$$
\text { 43. } \mathrm{h}_{\text {right }}=\mathrm{h}_{2 / 3} \text {. }
$$

End 


\section{If $\left(\mathrm{RSC}_{1 / 3}>\mathrm{RSC}_{2 / 3}\right)$}

Then

\section{Begin}

Comment: step 45 moved the left boundary to the right.

$$
\text { 45. } h_{\text {left }}=h_{1 / 3} \text {. }
$$

\section{End}

\section{End}

Comment: steps 46 - 55 verified that the left boundary and the right boundary approximated the $\mathrm{h}$ bandwidth which generated the minimum residual squares criterion error estimate in the residual squares criterion versus bandwidth curve.

46. $\mathrm{RSC}_{\text {left }}=\mathrm{RSC}\left(\mathrm{h}_{\mathrm{left}}\right)$.

47. $\mathrm{RSC}_{\text {right }}=\mathrm{RSC}\left(\mathrm{h}_{\text {right }}\right)$.

48. $\mathrm{RSC}_{\text {saved left }}=\mathrm{RSC}\left(\mathrm{h}_{\text {saved left }}\right)$.

49. $\mathrm{RSC}_{\text {saved right }}=\mathrm{RSC}\left(\mathrm{h}_{\text {saved right }}\right)$.

50. If $\left(\left(\mathrm{h}_{\text {saved left }} \leq \mathrm{h}_{\text {left }}\right)\right.$ and $\left(\mathrm{h}_{\text {left }} \leq \mathrm{h}_{\text {saved right }}\right)$ and $\left(\mathrm{RSC}_{\text {left }} \leq \mathrm{RSC}_{\text {saved left }}\right)$ and $\left(\mathrm{RSC}_{\text {left }} \leq\right.$ $\left.\mathrm{RSC}_{\text {saved right }}\right)$ )

Then

\section{Begin}

51. correct.

End

Else

\section{Begin}

52. output error.

End

53. If $\left(\left(h_{\text {saved left }} \leq \mathrm{h}_{\text {right }}\right)\right.$ and $\left(\mathrm{h}_{\text {right }} \leq \mathrm{h}_{\text {saved right }}\right)$ and $\left(\mathrm{RSC}_{\text {right }} \leq \mathrm{RSC}_{\text {saved left }}\right)$ and $\left(\mathrm{RSC}_{\text {right }} \leq\right.$ $\left.\mathrm{RSC}_{\text {saved right}}\right)$ )

Then

$$
\text { Begin }
$$

54. correct. 


\section{End}

Else

\section{Begin}

55. output error.

End

Comment: steps 56 - 57 computed the average of the left boundary and the right boundary. The average approximated the $\mathrm{h}$ bandwidth which generated the minimum residual squares criterion error estimate in the residual squares criterion versus bandwidth curve.

56. $h_{\text {average }}=\left(\left(h_{\text {left }}+h_{\text {right }}\right) / 2\right)$.

57. Output the $h_{\text {average }}$

$h_{1 / 3}=1 / 3$ intermediate point between the left boundary and the right boundary of the $h$ bandwidth.

$h_{2 / 3}=2 / 3$ intermediate point between the left boundary and the right boundary of the $h$ bandwidth.

$h_{\text {average }}=$ average of the left boundary and the right boundary of the $h$ bandwidth.

$\mathrm{h}_{\text {initial }}=$ initial $\mathrm{h}$ bandwidth.

$\mathrm{h}_{\text {left }}=$ left boundary of the $\mathrm{h}$ bandwidth.

$\mathrm{h}_{\text {new }}=$ next estimate of the $\mathrm{h}$ bandwidth.

$\mathrm{h}_{\mathrm{old}}=$ previous estimate of the $\mathrm{h}$ bandwidth.

$h_{\text {right }}=$ right boundary of the $h$ bandwidth.

$h_{\text {saved left }}=$ saved left boundary of the $h$ bandwidth.

$\mathrm{h}_{\text {saved right }}=$ saved right boundary of the $\mathrm{h}$ bandwidth.

$\mathrm{n}=$ number of datapoint set elements.

$\mathrm{RSC}_{1 / 3}=$ residual squares criterion error estimate of the $h_{1 / 3}$ bandwidth.

$\mathrm{RSC}_{2 / 3}=$ residual squares criterion error estimate of the $\mathrm{h}_{2 / 3}$ bandwidth.

$\mathrm{RSC}_{\text {left }}=$ residual squares criterion error estimate of the $\mathrm{h}_{\text {left }}$ bandwidth.

$\mathrm{RSC}_{\text {new }}=$ residual squares criterion error estimate of the $\mathrm{h}_{\text {new }}$ bandwidth.

$\mathrm{RSC}_{\text {old }}=$ residual squares criterion error estimate of the $\mathrm{h}_{\mathrm{old}}$ bandwidth.

$\mathrm{RSC}_{\text {right }}=$ residual squares criterion error estimate of the $\mathrm{h}_{\text {right }}$ bandwidth . 
$\mathrm{RSC}_{\text {saved left }}=$ saved residual squares criterion error estimate of the $\mathrm{h}_{\mathrm{left}}$ bandwidth.

$\mathrm{RSC}_{\text {saved right }}=$ saved residual squares criterion error estimate of the $h_{\text {right }}$ bandwidth .

tolerance $=$ difference between the $h_{\text {left }}$ bandwidth and the $h_{\text {right }}$ bandwidth .

tolerance $_{\text {absolute }}=$ absolute difference between the $h_{\text {left }}$ bandwidth and the $h_{\text {right }}$ bandwidth . tolerance minimum $=$ minimum difference between the $h_{\text {left }}$ bandwidth and the $h_{\text {right }}$ bandwidth.

tolerance $_{\text {relative }}=$ relative difference between the $h_{\text {left }}$ bandwidth and the $h_{\text {right }}$ bandwidth . $\mathrm{x}_{1}, \mathrm{x}_{2}, \mathrm{x}_{3}, \ldots \mathrm{x}_{\mathrm{n}}=\mathrm{x}$ datapoint set of $\mathrm{n}$ elements.

$\mathrm{x}_{\text {maximum }}=\mathrm{x}$ maximum datapoint.

$\mathbf{x}_{\text {minimum }}=\mathrm{x}$ minimum datapoint.

$\mathbf{x}_{\text {range }}=$ difference between the $\mathrm{x}$ maximum datapoint and the $\mathrm{x}$ minimum datapoint.

\subsubsection{1 $\mathrm{H}_{1, \text { mean Algorithm }}$}

The $h_{1}$ bandwidth was the scale parameter that controlled the amount of $x$ direction smoothing applied to the regression data. The $h_{1, \text { mean }}$ algorithm computed the $h_{1 \text {,mean }}$ bandwidth which was the optimal choice of the $h_{1}$ bandwidth for the regression mean estimation. The $h_{1, \text { mean }}$ algorithm subjected the gene transcription measurements to a series of six calculations:

1. The input consisted of the set of $x_{1}, x_{2}, x_{3}, \ldots x_{n}$ datapoints.

Comment: step 2 determined the existence of the optimal $h_{1, \text { mean }}$ bandwidth.

2. minimum_present $=h_{1}$, minimum_present .

3. If (minimum_present $=$ true)

Then

Begin

Comment: step 4 computed the optimal $\mathrm{h}_{1, \text { mean }}$ bandwidth.

4. $\mathrm{h}_{1, \text { mean }}=\mathrm{h}_{1, \text { expansion-contraction }}$.

End

Else

Begin 
Comment: step 5 computed the suboptimal $\mathrm{h}_{1, \text { mean }}$ bandwidth.

5. $\mathrm{h}_{1, \text { mean }}=\left(\left(\mathrm{x}_{\mathrm{n}}-\mathrm{x}_{1}\right) / 50\right)$.

End

6. Output the $h_{1, \text { mean }}$

$h_{1, \text { mean }}=$ optimal choice of the $h_{1}$ bandwidth for the regression mean estimation.

minimum_present $=$ Boolean variable which indicated whether the minimum residual squares criterion error estimate existed in the residual squares criterion versus bandwidth curve.

$\mathrm{n}=$ number of datapoint set elements.

$\mathrm{x}_{1}, \mathrm{x}_{2}, \mathrm{x}_{3}, \ldots \mathrm{x}_{\mathrm{n}}=\mathrm{x}$ datapoint set of $\mathrm{n}$ elements.

\subsubsection{2 $\mathrm{H}_{1, \mathrm{p}}$ Algorithm}

The $h_{1}$ bandwidth was the scale parameter that controlled the amount of $x$ direction smoothing applied to the regression data. The $h_{1, p}$ bandwidth was the scale parameter that controlled the amount of $\mathrm{x}$ direction smoothing applied to the regression

data for the $p$ percentile. The $h_{1, p}$ algorithm subjected the $h_{1, \text { mean }}$ bandwidth and the $p$ percentile to a series of four calculations:

1. The input consisted of the $h_{1, \text { mean }}$ bandwidth and the $p$ percentile.

2. $\mathrm{p}=(0.01 * \mathrm{p})$.

3. $\mathrm{h}_{1, \mathrm{p}}=\left(\mathrm{h}_{1, \text { mean }} * 16 *\left((\mathrm{p} *(1-\mathrm{p})) /\left(\phi\left(\Phi^{-1}(\mathrm{p})\right)^{2}\right)\right)^{0.2}\right)$

4. Ouput the $h_{1, p}$.

$\mathrm{h}_{1 \text {,mean }}=$ optimal choice of the $\mathrm{h}_{1}$ bandwidth for the regression mean estimation.

$\mathrm{h}_{1, \mathrm{p}}=$ bandwidth which was the scale parameter that controlled the amount of $\mathrm{x}$ direction smoothing applied to the regression data for the $\mathrm{p}$ percentile.

$\mathrm{p}=$ percentile.

$\phi=$ standard normal density function.

$\Phi=$ standard normal distribution function. 


\subsubsection{3 $\mathrm{H}_{2, \mathrm{p}}$ Algorithm}

The $h_{2}$ bandwidth was the scale parameter that controlled the amount of $y$ direction smoothing applied to the regression data. The $h_{2, p}$ bandwidth was the scale parameter that controlled the amount of y direction smoothing applied to the regression data for the p percentile. The $h_{2, p}$ algorithm subjected the $h_{1,0.50}$ bandwidth and the $h_{1, p}$ bandwidth to a series of seven calculations:

1. The input consisted of the $h_{1,0.50}$ bandwidth and the $h_{1, p}$ bandwidth.

2. If $\left(\mathrm{h}_{1,0.50}<1\right)$

Then

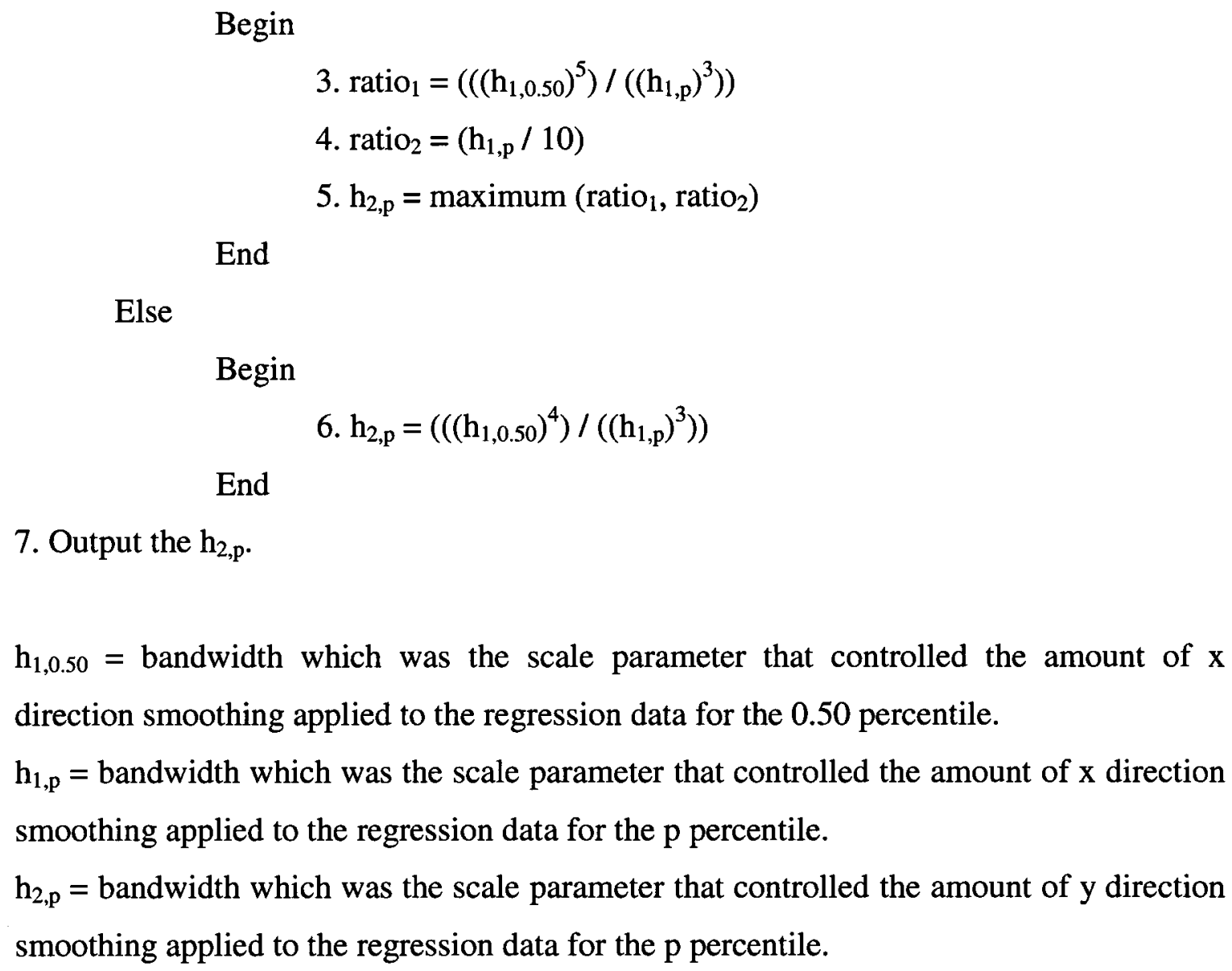

7. Output the $\mathrm{h}_{2, \mathrm{p}}$.

\subsubsection{Local Linear Kernel Estimation Function}


The local linear kernel estimation function $\mathrm{m}$ was the nonparametric estimate of the conditional mean observation. The local linear kernel estimation function $\mathrm{m}$ was defined:

$\mathrm{m}=\left(\left(\Sigma_{\mathrm{j}} \mathrm{w}_{\mathrm{j}}\left(\mathrm{x}_{\text {grid }}\right)\right)^{-1} *\left(\Sigma_{\mathrm{j}} \mathrm{w}_{\mathrm{j}}\left(\mathrm{x}_{\text {grid }}\right) * \mathrm{y}_{\text {data } \mathrm{j}} \mathrm{j}\right)\right.$

$\mathrm{w}_{\mathrm{j}}=$ weight $\mathrm{j}$.

$\mathrm{x}_{\text {grid }}=\mathrm{x}$ gridpoint.

$\mathrm{y}_{\text {data,j }}=\mathrm{y}$ datapoint $\mathrm{j}$.

$\mathrm{m}=$ local linear kernel estimation function value.

\subsubsection{Implicit Estimation Function}

The implicit estimation function computed the successive approximation of the nonparametric quantile regression. The implicit estimation function was defined:

$$
\begin{aligned}
& \mathrm{q}_{\text {new }, \mathrm{p}}\left(\mathrm{x}_{\text {grid }}\right)=\left(\left(\mathrm{m}\left(\mathrm{x}_{\text {grid }}\right)\right)+\left(((2 * \mathrm{p})-1) * \mathrm{~h}_{2, \mathrm{p}}\right)+\left(\left(( \Sigma _ { \mathrm { j } } ( ( \mathrm { q } _ { \text { old } , \mathrm { p } } ( \mathrm { x } _ { \text { grid } } ) ) - \mathrm { y } _ { \text { data,j } } - \mathrm { h } _ { 2 , \mathrm { p } } ) ) * \left(\mathrm{w}_{\mathrm{j}}\right.\right.\right.\right. \\
& \left.\left.\left.\left(\mathrm{x}_{\text {grid }}\right)\right) *\left(\mathrm{I}\left(\mathrm{y}_{\text {data,j }} \leq\left(\left(\mathrm{q}_{\text {old }, \mathrm{p}}\left(\mathrm{x}_{\text {grid }}\right)\right)-\mathrm{h}_{2, \mathrm{p}}\right)\right)\right)\right) /\left(\Sigma_{\mathrm{j}}\left(\mathrm{w}_{\mathrm{j}}\left(\mathrm{x}_{\text {grid }}\right)\right)\right)\right)+\left(\left(\left(\Sigma _ { \mathrm { j } } \left(\left(\mathrm{q}_{\text {old }, \mathrm{p}}\left(\mathrm{x}_{\text {grid }}\right)\right)-\mathrm{y}_{\text {data }, \mathrm{j}}+\right.\right.\right.\right. \\
& \left.\left.\left.\left.\left.\mathrm{h}_{2, \mathrm{p}}\right)\right) *\left(\mathrm{w}_{\mathrm{j}}\left(\mathrm{x}_{\text {grid }}\right)\right) *\left(\mathrm{I}\left(\mathrm{y}_{\text {data }, \mathrm{j}} \geq\left(\left(\mathrm{q}_{\text {old }, \mathrm{p}}\left(\mathrm{x}_{\text {grid }}\right)\right)+\mathrm{h}_{2, \mathrm{p}}\right)\right)\right)\right) /\left(\Sigma_{\mathrm{j}}\left(\mathrm{w}_{\mathrm{j}}\left(\mathrm{x}_{\text {grid }}\right)\right)\right)\right)\right)
\end{aligned}
$$

$\mathrm{h}_{2, \mathrm{p}}=$ bandwidth which was the scale parameter that controlled the amount of $\mathrm{y}$ direction smoothing applied to the regression data for the p percentile.

$\mathrm{I}=$ identity function.

$\mathrm{m}\left(\mathrm{x}_{\text {grid }}\right)=$ local linear kernel estimate at the $\mathrm{x}_{\text {grid }}$ point.

$\mathrm{p}=$ percentile.

$\mathrm{q}_{\text {new,p }}\left(\mathrm{x}_{\text {grid }}\right)=$ next estimate of the $\mathrm{p}$ percentile at the $\mathrm{x}_{\text {grid }}$ point.

$\mathrm{q}_{\mathrm{old}, \mathrm{p}}\left(\mathrm{x}_{\text {grid }}\right)=$ previous estimate of the $\mathrm{p}$ percentile at the $\mathrm{x}_{\text {grid }}$ point.

$w_{j}\left(x_{\text {grid }}\right)=$ weight between the $x_{\text {grid }}$ point and the $x_{\text {data,j }}$ point.

$\mathrm{x}_{\text {data,j }}=\mathrm{x}$ datapoint $\mathrm{j}$.

$\mathrm{x}_{\text {grid }}=\mathrm{x}$ gridpoint. 
$\mathrm{y}_{\text {data.j }}=\mathrm{y}$ datapoint $\mathrm{j}$.

\subsubsection{Quantile Tolerance Function}

The quantile tolerance function computed the relative difference between the next estimate of the y grid point and the previous estimate of the y grid point. The quantile tolerance function was defined:

tolerance $=\left(\left(\right.\right.$ absolute $\left.\left(\mathrm{y}_{\text {grid,new }}-\mathrm{y}_{\text {grid,old }}\right)\right) /\left(\right.$ absolute $\left.\left.\left(\mathrm{y}_{\text {grid,old }}\right)\right)\right)$

tolerance $=$ relative difference between the next estimate of the y gridpoint and the previous estimate of the y gridpoint.

$\mathrm{y}_{\text {grid,old }}=$ previous estimate of the $\mathrm{y}$ gridpoint.

$\mathrm{y}_{\text {grid,new }}=$ next estimate of the $\mathrm{y}$ gridpoint.

\subsubsection{Nonparametric Quantile Regression Algorithm}

The nonparametric quantile regression algorithm computed the final values of the quantiles. The nonparametric quantile regression algorithm subjected the gene transcription measurements to a series of eleven calculations:

1. The input consisted of the set of $x_{1}, x_{2}, x_{3}, \ldots x_{n}$ datapoints and the set of $y_{1}, y_{2}, y_{3}, \ldots$ $\mathrm{y}_{\mathrm{n}}$ datapoints which were coordinately joined to form the set of ordered pairs: $\left(\mathrm{x}_{1}, \mathrm{y}_{1}\right),\left(\mathrm{x}_{2}\right.$, $\left.y_{2}\right),\left(x_{3}, y_{3}\right), \ldots\left(x_{n}, y_{n}\right)$. The input also consisted of the ten $x$ grid points: $x_{\text {grid, }, 1}, x_{\text {grid,2, }}$, $\mathrm{x}_{\text {grid }, 3}, \ldots \mathrm{x}_{\text {grid, }, 10}$.

2. The quantile initialization algorithm computed the initial values of the quantiles. The ten $\mathrm{x}$ grid points $\mathrm{x}_{\text {grid, }, 1}, \mathrm{x}_{\text {grid, }, 2}, \mathrm{x}_{\text {grid,3, }}, \ldots \mathrm{x}_{\text {grid, } 10}$ each corresponded to ninety nine $\mathrm{y}$ grid points $y_{\text {grid,i, }, 1} y_{\text {grid,i, }, 2}, y_{\text {grid,i,3, }}, \ldots y_{\text {grid,i,99 }}$ which formed the total of nine hundred ninety $y$ grid points: $y_{\text {grid, } 1,1}, y_{\text {grid, } 1,2}, y_{\text {grid, } 1,3}, \ldots$ ggrid,10,99.

Comment: steps 3 - 10 computed the final values of the quantiles for the nonparametric quantile regression using repeated iterations of the implicit estimation function. 
3. Iterate $\mathrm{i}$ from 1 through 10

Begin

4. Iterate $\mathrm{j}$ from 1 through 99

Begin

5. $\mathrm{y}_{\text {grid, }, \mathrm{old}}=\mathrm{y}_{\text {grid, }, \mathrm{i}, \mathrm{j}}$

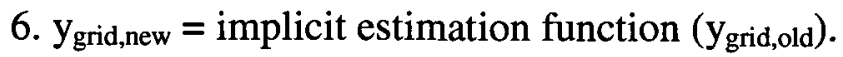

7. While ((quantile tolerance function ( $\left.\left.y_{\text {grid,old, }} y_{\text {grid,new }}\right)\right)>$

$0.0001)$

Begin

8. $\mathrm{y}_{\text {grid,old }}=\mathrm{y}_{\text {grid,new }}$.

9. $\mathrm{y}_{\text {grid,new }}=$ implicit estimation function

( $\left.\mathrm{y}_{\text {grid,old }}\right)$.

End

10. $\mathrm{y}_{\text {grid,i,j }}=\mathrm{y}_{\text {grid,new }}$.

End

End

11. Output the final values of the quantiles which corresponded to the nine hundred ninety y grid points: $y_{\text {grid,1,1 }}, y_{\text {grid, } 1,2}, y_{\text {grid, }, 1,3}, \ldots y_{\text {grid,10,99. }}$

$\mathrm{n}=$ number of datapoint set elements.

$\mathrm{x}_{1}, \mathrm{x}_{2}, \mathrm{x}_{3}, \ldots \mathrm{x}_{\mathrm{n}}=\mathrm{x}$ datapoint set of $\mathrm{n}$ elements.

$\mathrm{x}_{\text {grid,1 }}, \mathrm{x}_{\text {grid,2 }}, \mathrm{x}_{\text {grid,3 }}, \ldots \mathrm{x}_{\text {grid,10 }}=\mathrm{x}$ gridpoint set of 10 elements.

$y_{1}, y_{2}, x_{3}, \ldots y_{n}=y$ datapoint set of $n$ elements.

$\mathrm{y}_{\text {grid, }, \mathrm{i}, \mathrm{j}}=\mathrm{y}$ gridpoint $\mathrm{i} \mathrm{j}$ in which $\mathrm{i}$ corresponded to the $\mathrm{x}$ gridpoint $\mathrm{i}$ and $\mathrm{j}$ corresponded to the percentile $\mathrm{j}$.

$\mathrm{y}_{\text {grid,new }}=$ next estimate of the $\mathrm{y}$ gridpoint.

$\mathrm{y}_{\text {grid,old }}=$ previous estimate of the $\mathrm{y}$ gridpoint.

$\mathrm{y}_{\text {grid, } 1,1}, \mathrm{y}_{\text {grid, } 1,2,}, \mathrm{y}_{\text {grid,1,3, }}, \ldots \mathrm{y}_{\text {grid,10,99 }}=\mathrm{y}$ gridpoint set of 990 elements.

$\left(x_{1}, y_{1}\right),\left(x_{2}, y_{2}\right),\left(x_{3}, y_{3}\right), \ldots\left(x_{n}, y_{n}\right)=(x, y)$ datapoint set of $n$ ordered pairs.

\subsection{Differential Gene Expression (DGE) Algorithm Logical Structure}


The novel Differential Gene Expression (DGE) algorithm was the proposed method for the solution of the Differential Gene Expression (DGE) problem. The DGE algorithm subjected the gene transcription measurements in the three control state microarray datasets and the three experimental state microarray datasets to a series of eighteen calculations:

1. The $x$ values were evenly divided into nine partitions using ten $x$ grid points. The initial $\mathrm{x}$ value corresponded to the first $\mathrm{x}$ grid point. The final $\mathrm{x}$ value corresponded to the tenth $x$ grid point.

2. At each of the ten $x$ grid points on the independent $x$ variable, the ninety nine quantiles were computed on the dependent y variable. The ninety nine quantiles were computed using the nonparametric quantile regression algorithm (Yu and Jones 1998). The ninety nine quantiles corresponded to the ninety nine y grid points.

3. The ten $x$ grid points and the ninety nine $y$ grid points formed the total of nine hundred ninety grid points. The grid points were interconnected with lines along the twodimensional Cartesian axes to form the two-dimensional Cartesian geometrical grid.

4. The Cartesian coordinate ordered pair data values $(x, y)$ were superimposed onto the two-dimensional Cartesian geometrical grid.

5. The initial quantiles included the quantiles which were lesser than the 0.01 quantile, $\mathrm{q}_{\text {initial }}<0.01$. The Cartesian coordinate ordered pair data values $(\mathrm{x}, \mathrm{y})$ and the twodimensional Cartesian geometrical grid used linear extrapolation to compute the preliminary initial quantiles.

$$
\mathrm{q}_{\text {initial }}=\left(\left(\left(\mathrm{q}_{1}(\mathrm{x})\right)-\mathrm{y}\right) /\left(\left(\mathrm{q}_{2}(\mathrm{x})\right)-\left(\mathrm{q}_{1}(\mathrm{x})\right)\right)\right)
$$


6. The initial quantiles throughout all of the $C(6,3)=20$ combinations of the six microarray datasets consisting of the three control microarray datasets and the three experimental microarray datasets were searched for the maximum initial quantile, $\mathrm{q}_{\text {initial, }}$ max.

7. The maximum initial quantile $q_{\text {initial, }}$ max was used to compute the revised initial quantiles.

$\mathrm{q}_{\text {initial }}=\mathrm{q}_{\text {initial }} / \mathrm{q}_{\text {initial, } \max }$

8. The intermediate quantiles included the quantiles which were greater than or equal to the 0.01 quantile and lesser than or equal to the 0.99 quantile, $0.01 \leq$ qintermediate $\leq 0.99$. The Cartesian coordinate ordered pair data values $(\mathrm{x}, \mathrm{y})$ and the two-dimensional Cartesian geometrical grid used linear interpolation to compute the intermediate quantiles.

$\mathrm{q}_{\text {intermediate }}=\left(\left(\left(\mathrm{y}-\left(\mathrm{q}_{\mathrm{j}}(\mathrm{x})\right)\right) /\left(\left(\mathrm{q}_{\mathrm{j}+1}(\mathrm{x})\right)-\left(\mathrm{q}_{\mathrm{j}}(\mathrm{x})\right)\right)\right)+\left(\mathrm{q}_{\mathrm{j}}(\mathrm{x})\right)\right)$

9. The final quantiles included the quantiles which were greater than the 0.99 quantile, $0.99<\mathrm{q}_{\text {final }}$. The Cartesian coordinate ordered pair data values $(\mathrm{x}, \mathrm{y})$ and the twodimensional Cartesian geometrical grid used linear extrapolation to compute the preliminary final quantiles.

$\mathrm{q}_{\text {final }}=\left(\left(\mathrm{y}-\left(\mathrm{q}_{99}(\mathrm{x})\right)\right) /\left(\left(\mathrm{q}_{99}(\mathrm{x})\right)-\left(\mathrm{q}_{98}(\mathrm{x})\right)\right)\right)$

10. The final quantiles throughout all of the $C(6,3)=20$ combinations of the six microarray datasets consisting of the three control microarray datasets and the three experimental microarray datasets were searched for the maximum final quantile, $q_{\text {final, } \max }$.

11. The maximum final quantile $q_{\text {initial, }}$ max was used to compute the revised final quantiles. 
$\mathrm{q}_{\text {final }}=\mathrm{q}_{\text {final }} / \mathrm{q}_{\text {final, max }}$

12. The expected quantiles were computed as the average quantiles arising from the $C$ (6, $3)=20$ combinations of the six microarray datasets consisting of the three control microarray datasets and the three experimental microarray datasets. The calculations one through thirteen were repeated twenty times.

13. The differentials were computed as the differences between the quantiles and the expected quantiles: for $\mathrm{i}=1$ to $\mathrm{n}, \mathrm{d}_{\mathrm{i}}=\mathrm{E}\left(\mathrm{q}_{\mathrm{i}}\right)-\mathrm{q}_{\mathrm{i}}$. Computations were performed for three reference differentials:

1. maximum positive differential.

2. minimum absolute differential.

3. maximum negative differential.

The maximum positive differential $d_{m p}=E\left(q_{m p}\right)-q_{m p}$ corresponded to the ordered pair $\left(q_{m p}, E\left(q_{m p}\right)\right)$.

The minimum absolute differential $d_{m a}=E\left(q_{m a}\right)-q_{m a}$ corresponded to the ordered pair $\left(\mathrm{q}_{\mathrm{ma}}, \mathrm{E}\left(\mathrm{q}_{\mathrm{ma}}\right)\right)$.

The maximum negative differential $\mathrm{d}_{\mathrm{mn}}=\mathrm{E}\left(\mathrm{q}_{\mathrm{mn}}\right)-\mathrm{q}_{\mathrm{mn}}$ corresponded to the ordered pair $\left(q_{m n}, E\left(q_{m n}\right)\right)$.

The three corresponding ordered pairs occurred on the basis of increasing quantile order: $\mathrm{q}_{\mathrm{mp}}<\mathrm{q}_{\mathrm{ma}}<\mathrm{q}_{\mathrm{mn}}$.

14. The maximum positive differential ordered pair $\left(\mathrm{q}_{\mathrm{mp}}, \mathrm{E}\left(\mathrm{q}_{\mathrm{mp}}\right)\right)$ and the maximum negative differential ordered pair $\left(\mathrm{q}_{\mathrm{mn}}, \mathrm{E}\left(\mathrm{q}_{\mathrm{mn}}\right)\right)$ were used as the basis for computing the linear regression equation which defined the revised expected quantiles: 
$\operatorname{rev}(E(q))=(($ slope $*(E(q)))+y$-intercept $)$

15. The revised expected quantiles were computed over three quantile ranges:

1. For the quantile range $0 \leq \mathrm{q}<\mathrm{q}_{\mathrm{mp}}$, rev $(\mathrm{E}(\mathrm{q}))=(($ slope $*(E(\mathrm{q})))+\mathrm{y}-$ intercept).

2. For the quantile range $\mathrm{q}_{\mathrm{mp}} \leq \mathrm{q} \leq \mathrm{q}_{\mathrm{mn}}$, $\operatorname{rev}(\mathrm{E}(\mathrm{q}))=\mathrm{E}(\mathrm{q})$.

3. For the quantile range $\mathrm{q}_{\mathrm{mn}}<\mathrm{q} \leq 1$, rev $(\mathrm{E}(\mathrm{q}))=(($ slope $*(\mathrm{E}(\mathrm{q})))+\mathrm{y}-$ intercept).

16. The differentials were computed as the absolute differences between the quantiles and the revised expected quantiles: for $\mathrm{i}=1$ to $n, \mathrm{~d}_{\mathrm{i}}=\operatorname{abs}\left(\mathrm{E}\left(\mathrm{q}_{\mathrm{i}}\right)-\mathrm{q}_{\mathrm{i}}\right)$.

17. The quantiles were sorted into ascending order.

18. The quantiles were categorized into three gene transcription classifications on the basis of three quantile ranges:

1. negative regulation gene transcription measurements.

2. neutral regulation gene transcription measurements.

3. positive regulation gene transcription measurements.

The negative regulation gene transcription measurements corresponded to the quantile range in which $0 \leq \mathrm{q} \leq \mathrm{q}_{\mathrm{mp}}$. The neutral regulation gene transcription measurements corresponded to the quantile range in which $\mathrm{q}_{\mathrm{mp}}<\mathrm{q}<\mathrm{q}_{\mathrm{mn}}$. The positive regulation gene transcription measurements corresponded to the quantile range in which $\mathrm{q}_{\mathrm{mn}} \leq \mathrm{q} \leq 1$.

The DGE algorithm was demonstrated using synthetic microarray datasets. The synthetic microarray datasets underwent artificial generation, $\log$ transformation, Cartesian coordinate calculation and normalization. The synthetic microarray datasets 
consisted of 0.03 normalized fraction upregulated genes, 0.94 normalized fraction neutral genes and 0.03 normalized fraction downregulated genes. Refer to the differential gene expression graph (Figure 7). The synthetic microarray datasets were subjected to the DGE algorithm computation. The DGE algorithm computation performed intermediate calculations which underwent graphical visualization. Refer to the DGE algorithm nonlinear regression quantile graphs (Figure 8). Refer to the DGE algorithm quantile graph and expected quantile graph (Figure 9). Refer to the DGE algorithm nonlinear regression 0.03 quantile graph and 0.97 quantile graph (Figure 10). The DGE algorithm computation output underwent graphical visualization. Refer to the DGE algorithm graph (Figure 11). 


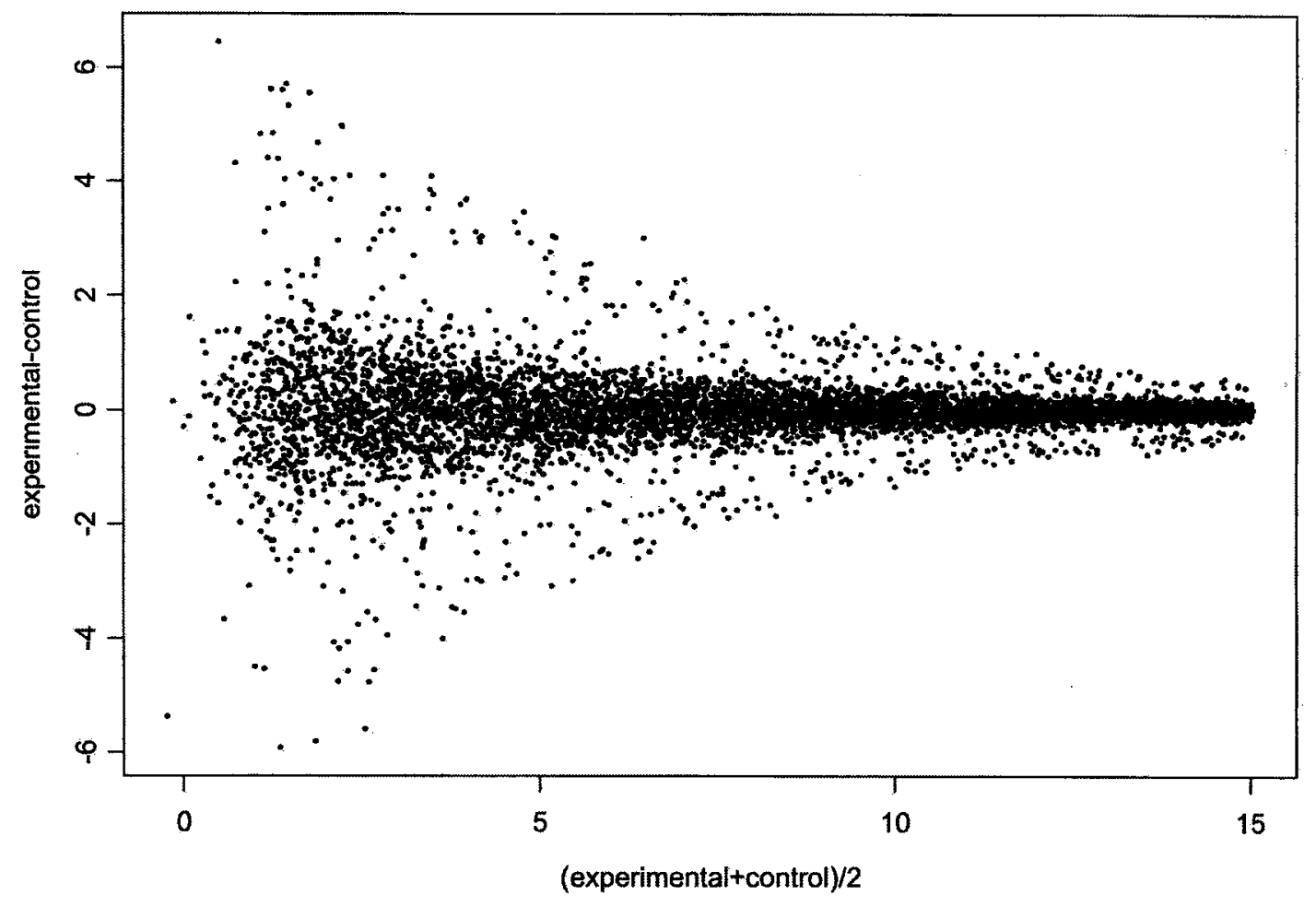

Figure 7. Differential gene expression graph. The datapoints included 0.03 normalized fraction upregulated genes, 0.94 normalized fraction neutral genes and 0.03 normalized fraction downregulated genes. 


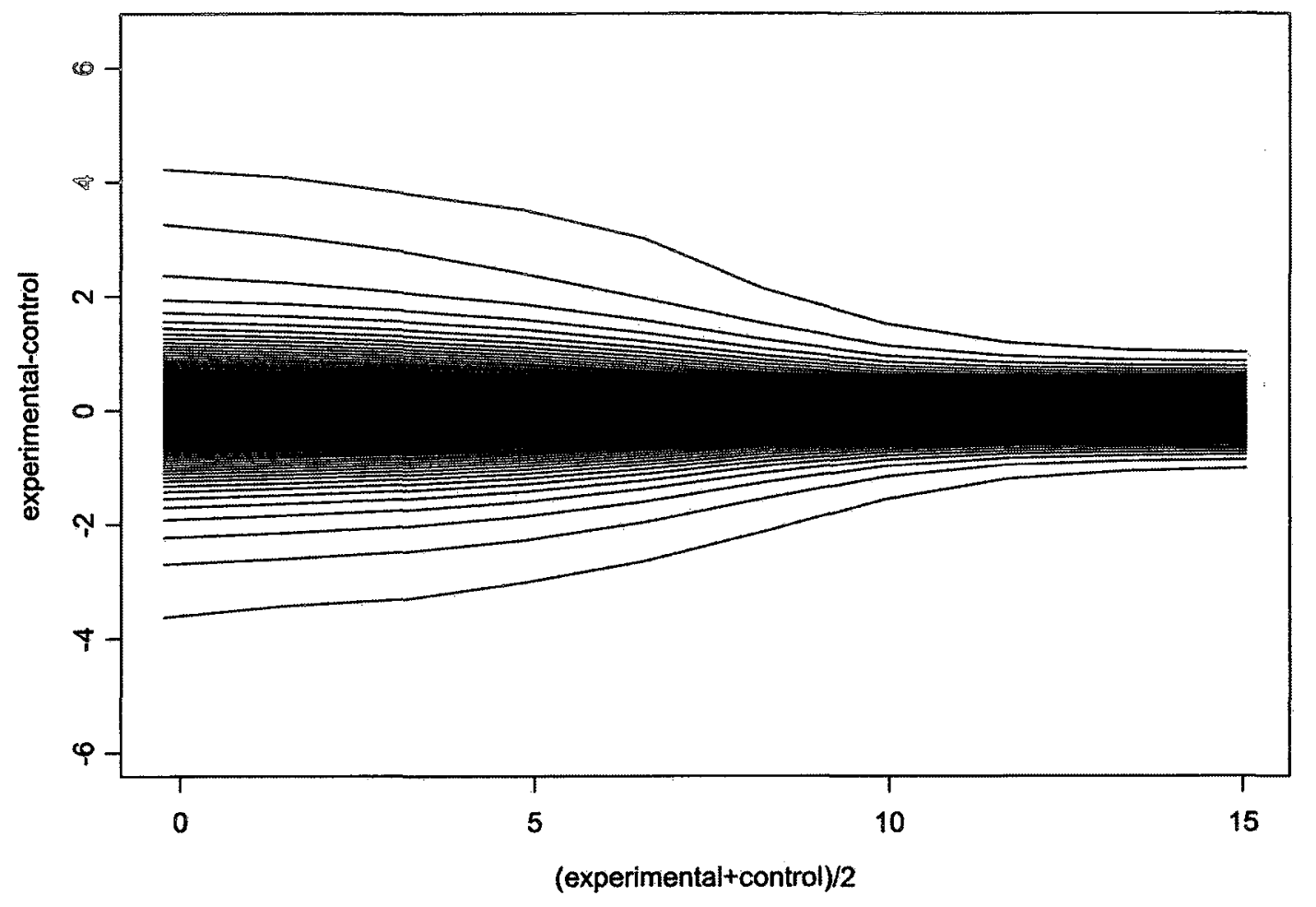

Figure 8. Differential Gene Expression (DGE) algorithm nonlinear regression quantile graphs. The ninety nine nonlinear regression quantile graphs were calculated using the Yu and Jones (1998) algorithm. The ninety nine nonlinear regression quantile graphs provided the basis for the identification of the upregulated genes and the downregulated genes. 


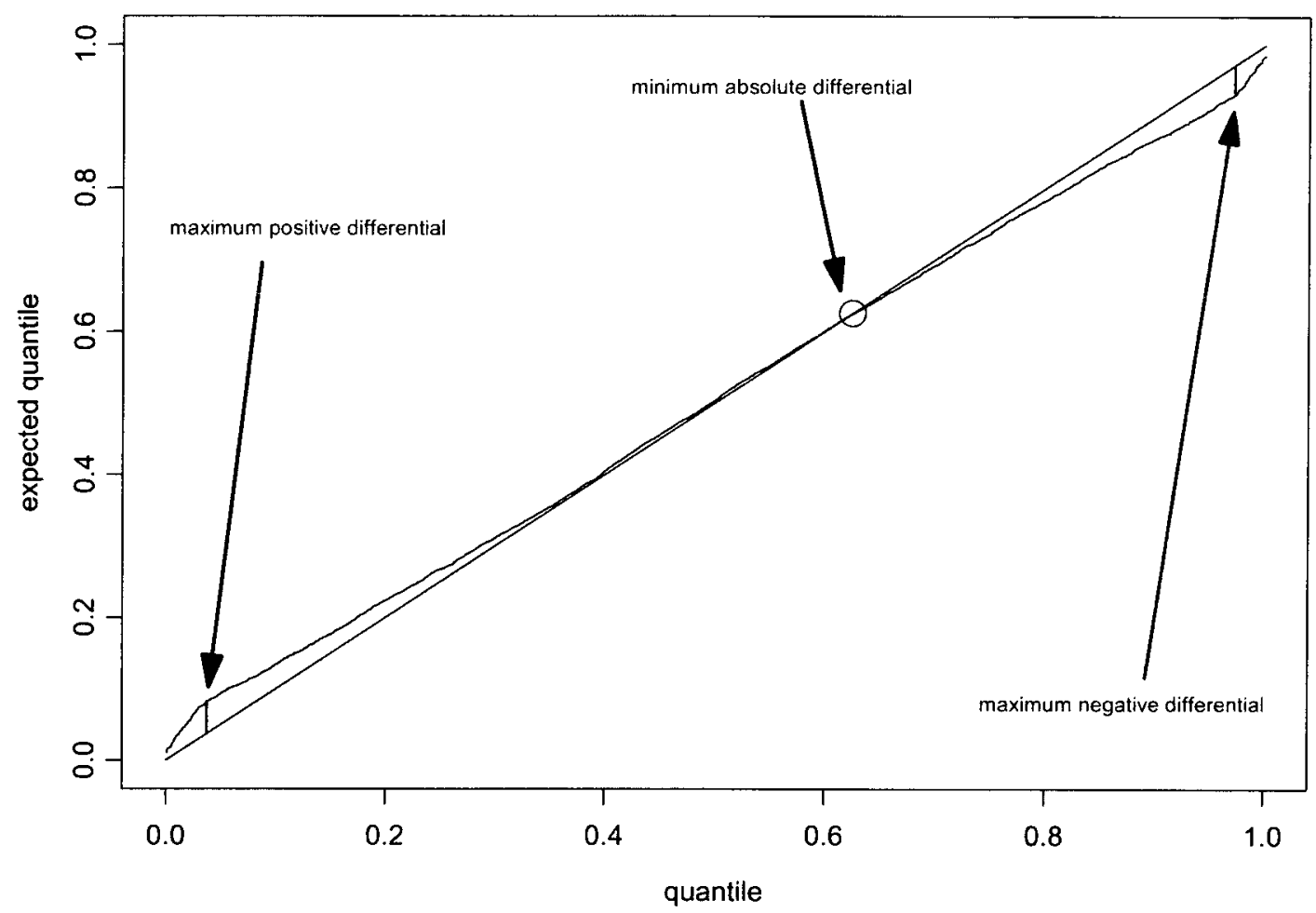

Figure 9. Differential Gene Expression (DGE) algorithm quantile graph and expected quantile graph. The quantile graph was represented as the straight black line. The expected quantile graph was represented as the curved black line. The maximum positive differential $\left(\mathrm{d}_{\mathrm{mp}}\right)$ was represented as the red line. The minimum absolute differential $\left(d_{m a}\right)$ was represented as the blue circle. The maximum negative differential $\left(d_{m n}\right)$ was represented as the green line. 


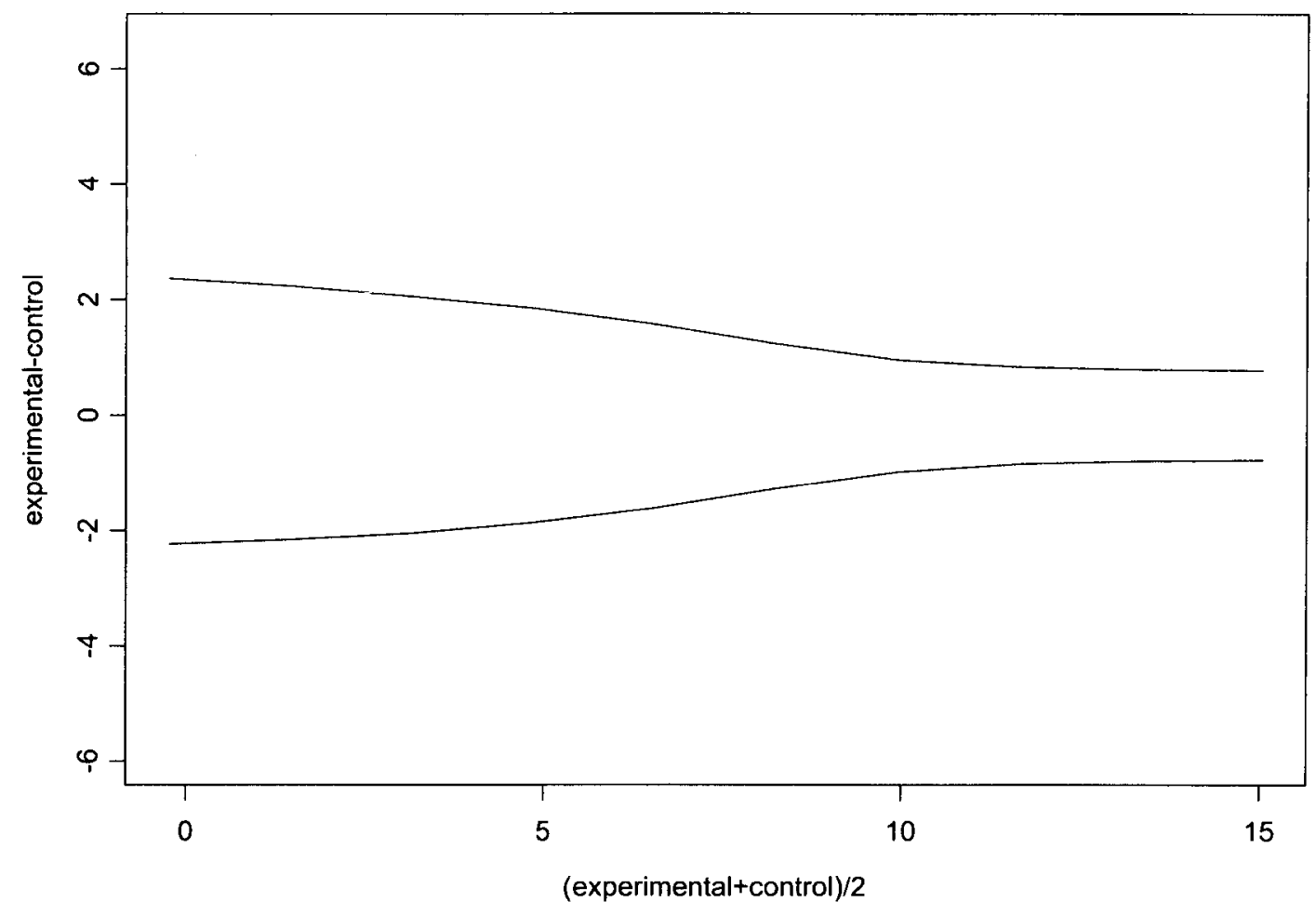

Figure 10. Differential Gene Expression (DGE) algorithm nonlinear regression 0.03 quantile graph and 0.97 quantile graph. The 0.03 quantile graph was represented as the red line. The 0.97 quantile graph was represented as the green line. 




Figure 11. Differential Gene Expression (DGE) algorithm graph. The 0.03 normalized fraction upregulated genes were represented as the green datapoints. The 0.94 normalized fraction neutral genes were represented as the black datapoints. The 0.03 normalized fraction downregulated genes were represented as the red datapoints. 


\section{Chapter 4 Experimental Analysis}

The experimental analysis determined the relative performance of the Significance Analysis of Microarrays (SAM) algorithm and the Differential Gene Expression (DGE) algorithm. The relative performance of the SAM algorithm and the DGE algorithm was based on four statistical procedures: 1. synthetic microarray datasets method, 2. confusion matrix classification statistics, 3. comparative accuracy performance analysis and 4. comparative precision performance analysis.

The synthetic microarray datasets method was used to generate the synthetic microarray datasets containing known differentially-expressed genes. The availability of the synthetic microarray datasets in which the numbers and the identities of the differentially-expressed genes were known in advance formed the basis for the comparative accuracy performance analysis.

The confusion matrix classification statistics were the methods which used the confusion matrix data to evaluate the classification system. The confusion matrix contained information about the actual classifications and the predicted classifications performed by the classification system (Kohavi and Provost 1998). The confusion matrix classification statistics were used to evaluate the DGE algorithm in the comparative accuracy performance analysis.

The comparative accuracy performance analysis was based on the use of the accuracy statistic. The accuracy statistic was defined as the degree of conformity of the computations or estimates to the exact or true values. The accuracy statistic was the closeness of an observation to the quantity intended to be observed. The comparative accuracy performance analysis was based on the analysis of microarray datasets in which the numbers and the identities of the differentially-expressed genes were known in advance. Microarray datasets containing known differentially-expressed genes do not exist. Therefore, it was necessary to use the synthetic microarray datasets method to 
generate the synthetic microarray datasets containing known differentially-expressed genes.

The comparative accuracy performance analysis consisted of twenty repeated experiments. In each experiment, the SAM algorithm computed the classifications of the differentially-expressed genes. The actual differentially-expressed genes and the predicted differentially-expressed genes were used to compute the SAM accuracy statistics. The SAM accuracy statistics were reported on the basis of individual experiments. In each experiment, the DGE algorithm computed the classifications of the differentially-expressed genes. The actual differentially-expressed genes and the predicted differentially-expressed genes were used to compute the DGE accuracy statistics. The DGE accuracy statistics were reported on the basis of individual experiments.

The advantage of the comparative accuracy performance analysis was that the accuracy statistics were the primary measurement for the determination of the relative performance of the SAM algorithm and the DGE algorithm. The disadvantage of the comparative accuracy performance analysis was that the accuracy statistics were generated through the use of the synthetic microarray datasets. The accuracy statistics generated through the use of the synthetic microarray datasets may not have been an accurate representation of the relative performance of the SAM algorithm and the DGE algorithm on the real microarray datasets.

The comparative precision performance analysis was based on the use of the precision statistic. The precision statistic was distinguished from the accuracy statistic. The precision statistic was defined as the spread of estimates of a parameter in a statistical model. The precision statistic was the quality associated with the class of measurements and referred to the way in which repeated observations conformed to themselves. The precision statistic referred to the dispersion of the observations whether or not the mean value around which the dispersion was measured approximated the true value. The comparative precision performance analysis was based on the analysis of 
microarray datasets containing differentially-expressed genes. The comparative precision performance analysis consisted of sixteen repeated experiments. In each experiment, the SAM algorithm computed the differential ranks of the differentially-expressed genes. The sixteen differential ranks generated by the sixteen SAM algorithm computations for each gene were used to compute the variances which formed the SAM precision statistics. The SAM precision statistics were reported on the basis of individual genes. In each experiment, the DGE algorithm computed the differential ranks of the differentially-expressed genes. The sixteen differential ranks generated by the sixteen DGE algorithm computations for each gene were used to compute the variances which formed the DGE precision statistics. The DGE precision statistics were reported on the basis of individual genes. The advantage of the comparative precision performance analysis was that the precision statistics were generated through the use of the real microarray datasets. The disadvantage of the comparative precision performance analysis was that the precision statistics were the secondary measurement for the determination of the relative performance of the SAM algorithm and the DGE algorithm.

\subsection{Synthetic Microarray Datasets Method}

The synthetic microarray datasets method was used to generate the synthetic microarray datasets containing known differentially-expressed genes. The availability of the synthetic microarray datasets in which the numbers and the identities of the differentially-expressed genes were known in advance formed the basis for the comparative accuracy performance analysis. The synthetic microarray datasets method consisted of the two component methods: 1. synthetic upregulated genes method and 2. synthetic downregulated genes method. The synthetic upregulated genes method transformed the natural microarray datasets. The randomly-chosen genes subset was subjected to the positive y value alteration. The positive $y$ value alteration produced the upregulated genes subset. The synthetic downregulated genes method transformed the natural microarray datasets. The randomly-chosen genes subset was subjected to the negative $y$ value alteration. The negative $y$ value alteration produced the downregulated genes subset. The synthetic upregulated genes method and the synthetic downregulated 
genes method transformed the natural microarray datasets to generate the synthetic microarray datasets. The synthetic microarray datasets consisted of the three subsets: 1 . upregulated genes, 2 . nonregulated genes and 3 . downregulated genes. The upregulated genes, the nonregulated genes and the downregulated genes formed three mutuallyexclusive subsets.

\subsubsection{Synthetic Upregulated Genes Method}

The synthetic upregulated genes method subjected the gene transcription measurements in the three control state microarray datasets and the three experimental state microarray datasets to a series of ten calculations:

1. The gene transcription measurements in the three control state microarray datasets and the three experimental state microarray datasets used the Differential Gene Expression (DGE) algorithm to compute the 0.99 quantile composite regression line.

2. The 0.05 normalized fraction or 330 randomly-chosen genes subset of the gene transcription measurements in the three control state microarray datasets were used to compute the control mean using the formulas:

$\log$ control $_{1}=\log \left(\right.$ control $\left._{1}\right)$

$\log$ control $_{2}=\log \left(\right.$ control $\left._{2}\right)$

$\log \operatorname{control}_{3}=\log \left(\operatorname{control}_{3}\right)$

control mean $=\left(\log\right.$ control $_{1}+\log$ control $_{2}+\log$ control $\left._{3}\right) / 3$

3. The 0.05 normalized fraction or 330 randomly-chosen genes subset of the gene transcription measurements in the three experimental state microarray datasets were used to compute the experimental mean using the formulas:

$\log$ experimental ${ }_{1}=\log \left(\right.$ experimental $\left._{1}\right)$

$\log$ experimental ${ }_{2}=\log \left(\right.$ experimental $\left.{ }_{2}\right)$ 
$\log$ experimental $_{3}=\log \left(\right.$ experimental $\left.{ }_{3}\right)$

experimental mean $=\left(\log\right.$ experimental $1+\log$ experimental $_{2}+\log$ experimental $\left._{3}\right) / 3$

4. The control mean and the experimental mean were used to compute the Cartesian $\mathrm{x}$ coordinate using the formula:

$\mathrm{x}=($ experimental mean + control mean $) / 2$

5. The Cartesian $\mathrm{x}$ coordinate and the 0.99 quantile composite regression line were used to compute the 0.99 quantile, $\mathrm{q}_{99}$.

6. The random number $r$ was randomly generated in the range: $0 \leq r \leq 1$.

7. The constant $s=1.3$ was initialized as the smallest multiplication factor for the upregulated genes. The constant $t=2.5$ was initialized as the largest multiplication factor for the upregulated genes.

8. The upregulated gene Cartesian y coordinate was randomly translated by $s$ to $t$ factors above the 0.99 quantile, $\mathrm{q}_{99}$. The transformed Cartesian y coordinate $\mathrm{y}_{\text {new }}$ was computed using the formula:

$\mathrm{y}_{\text {new }}=\left((\mathrm{t}-((\mathrm{t}-\mathrm{s}) *(\mathrm{sqrt}(\mathrm{r})))) * \mathrm{q}_{99}\right)$

9. The transformed control values were computed using the formulas:

$$
\begin{aligned}
& \text { control }_{1, \text { transformed }}=\text { control }_{1} * \mathrm{e}^{\left(-0.5 *\left((\text { control mean })-(\text { experimental mean })+\mathrm{Y}_{\text {new }}\right)\right)} \\
& \text { control }_{2, \text { transformed }}=\operatorname{control}_{2} * \mathrm{e}^{\left(-0.5 *\left((\text { control mean })-(\text { experimental mean })+\mathrm{Y}_{\text {new }}\right)\right)} \\
& \text { control }_{3, \text { transformed }}=\operatorname{control}_{3} * \mathrm{e}^{\left(-0.5 *\left((\text { control mean })-(\text { experimental mean })+\mathrm{Y}_{\text {new }}\right)\right)}
\end{aligned}
$$

10. The transformed experimental values were computed using the formulas: 
experimental ${ }_{1, \text { transformed }}=$ control $_{1} * \mathrm{e}^{\left(0.5 *\left((\text { control mean })-(\text { experimental mean })+\mathrm{Y}_{\text {new }}\right)\right)}$

experimental $_{2, \text { transformed }}=\operatorname{control}_{2} * \mathrm{e}^{\left(0.5 *\left((\text { control mean })-(\text { experimental mean })+Y_{\text {new }}\right)\right)}$

experimental 3 ,transformed $=\operatorname{control}_{3} * \mathrm{e}^{\left(0.5 *\left((\text { control mean })-(\text { experimental mean })+Y_{\text {new }}\right)\right)}$

\subsubsection{Synthetic Downregulated Genes Method}

The synthetic downregulated genes method subjected the gene transcription measurements in the three control state microarray datasets and the three experimental state microarray datasets to a series of ten calculations:

1. The gene transcription measurements in the three control state microarray datasets and the three experimental state microarray datasets used the Differential Gene Expression (DGE) algorithm to compute the 0.01 quantile composite regression line.

2. The 0.05 normalized fraction or 330 randomly-chosen genes subset of the gene transcription measurements in the three control state microarray datasets were used to compute the control mean using the formulas:

$\log$ control $_{1}=\log \left(\right.$ control $\left._{1}\right)$

$\log$ control $_{2}=\log \left(\operatorname{control}_{2}\right)$

$\log \mathrm{control}_{3}=\log \left(\mathrm{control}_{3}\right)$

control mean $=\left(\log\right.$ control $\left._{1}+\log \operatorname{control}_{2}+\log \operatorname{control}_{3}\right) / 3$

3. The 0.05 normalized fraction or 330 randomly-chosen genes subset of the gene transcription measurements in the three experimental state microarray datasets were used to compute the experimental mean using the formulas:

$\log$ experimental $_{1}=\log \left(\right.$ experimental $\left.{ }_{1}\right)$

$\log$ experimental ${ }_{2}=\log \left(\right.$ experimental $\left.{ }_{2}\right)$

$\log$ experimental $_{3}=\log \left(\right.$ experimental $\left.{ }_{3}\right)$

experimental mean $=\left(\log\right.$ experimental $1_{1}+\log$ experimental ${ }_{2}+\log$ experimental $\left.{ }_{3}\right) / 3$ 
4. The control mean and the experimental mean were used to compute the Cartesian $x$ coordinate using the formula:

$\mathrm{x}=($ experimental mean + control mean $) / 2$

5. The Cartesian $x$ coordinate and the 0.01 quantile composite regression line were used to compute the 0.01 quantile, $\mathrm{q}_{01}$.

6. The random number $r$ was randomly generated in the range: $0 \leq r \leq 1$.

7. The constant $s=1.3$ was initialized as the smallest multiplication factor for the downregulated genes. The constant $t=2.5$ was initialized as the largest multiplication factor for the downregulated genes.

8. The downregulated gene Cartesian y coordinate was randomly translated by $\mathrm{s}$ to $\mathrm{t}$ factors below the 0.01 quantile, $\mathrm{q}_{01}$. The transformed Cartesian y coordinate $\mathrm{y}_{\text {new }}$ was computed using the formula:

$\mathrm{y}_{\text {new }}=\left((\mathrm{t}-((\mathrm{t}-\mathrm{s}) *(\mathrm{sqrt}(\mathrm{r})))) * \mathrm{q}_{01}\right)$

9. The transformed control values were computed using the formulas:

$$
\begin{aligned}
& \text { control }_{1, \text { transformed }}=\text { control }_{1} * \mathrm{e}^{\left(-0.5 *\left((\text { control mean })-(\text { experimental mean })+\mathrm{Y}_{\text {new }}\right)\right)} \\
& \operatorname{control}_{2, \text { transformed }}=\operatorname{control}_{2} * \mathrm{e}^{\left(-0.5 *\left((\text { control mean })-(\text { experimental mean })+\mathrm{Y}_{\text {new }}\right)\right)} \\
& \text { control }_{3, \text { transformed }}=\operatorname{control}_{3} * \mathrm{e}^{\left(-0.5 *\left((\text { control mean })-(\text { experimental mean })+Y_{\text {new }}\right)\right)}
\end{aligned}
$$

10. The transformed experimental values were computed using the formulas:

$$
\begin{aligned}
& \text { experimental }_{1, \text { transformed }}=\operatorname{control}_{1} * \mathrm{e}^{\left(0.5 *\left((\text { control mean })-(\text { experimental mean })+Y_{\text {new }}\right)\right)} \\
& \text { experimental }_{2, \text { transformed }}=\operatorname{control}_{2} * \mathrm{e}^{\left(0.5 *\left((\text { control mean })-(\text { experimental mean })+Y_{\text {new }}\right)\right)}
\end{aligned}
$$


experimental 3 ,transformed $=\operatorname{control}_{3} * \mathrm{e}^{\left(0.5 *\left((\text { control mean })-(\text { experimental mean })+\mathrm{Y}_{\text {new }}\right)\right)}$

\subsection{Confusion Matrix Classification Statistics}

The confusion matrix contained information about the actual classifications and the predicted classifications performed by the classification system (Kohavi and Provost 1998). The classification system was evaluated using the confusion matrix data. The confusion matrix for the binary classification system is presented.

Table 1. Confusion matrix.

Actual Positive Value

Actual Negative Value

Predicted Positive Value True Positive

False Positive

Predicted Negative Value False Negative

True Negative

The confusion matrix classification performance analysis consisted of fourteen performance statistics: 1. true positive, 2. false positive, 3. true negative, 4. false negative, 5 . true positive rate, 6 . false positive rate, 7 . true negative rate, 8 . false negative rate, 9. positive predictive value, 10. negative predictive value, 11. accuracy, 12. geometric-mean $_{1}, 13$. geometric-mean 2 and 14. F-measure.

The true positive (TP) statistic was the number of predicted positive values which were in reality positive values. The TP statistic was equivalent to the number of hit values.

The false positive (FP) statistic was the number of predicted positive values which were in reality negative values. The FP statistic was equivalent to the number of false alarm values. The FP statistic was equivalent to the number of Type I errors. 
The true negative (TN) statistic was the number of predicted negative values which were in reality negative values. The TN statistic was equivalent to the number of correctly rejected values.

The false negative (FN) statistic was the number of predicted negative values which were in reality positive values. The FN statistic was equivalent to the number of missed values. The FN statistic was equivalent to the number of Type II errors.

The true positive rate (TPR) statistic was the proportion of actual positive values that were correctly classified as predicted positive values. The TPR statistic was equivalent to the sensitivity (SE) statistic. The TPR statistic was equivalent to the recall (RE) statistic. The TPR statistic was equivalent to the power (P) statistic. The formula for the TPR statistic was written as:

$\mathrm{TPR}=(\mathrm{TP} /(\mathrm{TP}+\mathrm{FN}))=\mathrm{SE}=\mathrm{RE}=\mathrm{P}$.

The false positive rate (FPR) statistic was the proportion of actual negative values that were incorrectly classified as predicted positive values. The FPR statistic was equivalent to the Type I error rate ( $\alpha$ ) statistic. The FPR statistic was equal to 1 minus the specificity (SP) statistic. The formula for the FPR statistic was written as:

$F P R=(F P /(T N+F P))=\alpha=(1-S P)$.

The true negative rate (TNR) statistic was the proportion of actual negative values that were correctly classified as predicted negative values. The TNR statistic was equivalent to the specificity (SP) statistic. The formula for the TNR statistic was written as:

$\mathrm{TNR}=(\mathrm{TN} /(\mathrm{TN}+\mathrm{FP}))=\mathrm{SP}$. 
The false negative rate (FNR) statistic was the proportion of actual positive values that were incorrectly classified as predicted negative values. The FNR statistic was equivalent to the Type II error rate $(\beta)$ statistic. The FNR statistic was equal to 1 minus the sensitivity (SE) statistic. The formula for the FNR statistic was written as:

$\mathrm{FNR}=(\mathrm{FN} /(\mathrm{TP}+\mathrm{FN}))=\beta=(1-\mathrm{SE})$

The positive predictive value (PPV) statistic was the proportion of predicted positive values that were correctly classified as actual positive values. The PPV statistic was equivalent to the precision (PR) statistic. The formula for the PPV statistic was written as:

$\mathrm{PPV}=(\mathrm{TP} /(\mathrm{TP}+\mathrm{FP}))=\mathrm{PR}$

The negative predictive value (NPV) statistic was the proportion of predicted negative values that were correctly classified as actual negative values. The formula for the NPV statistic was written as:

$\mathrm{NPV}=(\mathrm{TN} /(\mathrm{TN}+\mathrm{FN}))$

The accuracy (AC) statistic was the proportion of total values that were correctly classified. The formula for the AC statistic was written as:

$\mathrm{AC}=((\mathrm{TP}+\mathrm{TN}) /(\mathrm{TP}+\mathrm{FP}+\mathrm{TN}+\mathrm{FN}))$

The AC statistic may not be an adequate performance measurement when imbalanced classes arise in which the number of negative cases was much larger than the number of positive cases (Kubat et al. 1998). Consider a set of 1000 values that consisted of 995 actual negative values and 5 actual positive values. If the classification system classified all of the values as predicted negative values, then the accuracy would be 0.995 normalized fraction even though the classification system missed all of the actual positive 
values. Alternative accuracy performance measurements have been developed which compensate for imbalanced classes. The alternative accuracy performance measurements were based on the strategy of incorporating the TPR statistic within a product. The TPR statistic product strategy acted as a safeguard against the case in which all of the actual positive cases were classified incorrectly. In the event that all of the actual positive cases were classified incorrectly, then the alternative accuracy performance measurements would return a value of 0 . Three alternative accuracy performance measurements have been proposed: 1 . geometric-mean 1,2 . geometric-mean ${ }_{2}$ and 3. F-measure.

The geometric-mean 1 (GM1) statistic was the geometric mean of the TPR statistic and the PPV statistic (Kubat et al. 1998). The formula for the GM1 statistic was written as:

$\mathrm{GM} 1=(\mathrm{TPR} * \mathrm{PPV})^{0.5}$

The geometric-mean ${ }_{2}$ (GM2) statistic was the geometric mean of the TPR statistic and the TNR statistic (Kubat et al. 1998). The formula for the GM2 statistic was written as:

$\mathrm{GM} 2=(\mathrm{TPR} * \mathrm{TNR})^{0.5}$

The F-measure (FM) statistic was the weighted harmonic mean of the TPR statistic and the PPV statistic. The FM statistic assigned a weight $\beta$ to control the relative contributions of the TPR statistic and the PPV statistic in computing the weighted harmonic mean. The weight $\beta$ can adopt a value from 0 to infinity. The formula for the FM statistic given the weight $\beta$ was written as:

$\mathrm{FM}=\left(\left(\left(\beta^{2}+1\right) * \mathrm{PPV} * \mathrm{TPR}\right) /\left(\beta^{2} * \mathrm{PPV}+\mathrm{TPR}\right)\right)$

The $\mathrm{F}_{1}$-measure (F1M) statistic was the harmonic mean of the TPR statistic and the PPV statistic. The F1M statistic assigned a weight $\beta=1$ to designate the equal 
contributions of the TPR statistic and the PPV statistic in computing the harmonic mean. The formula for the F1M statistic given the weight $\beta=1$ was written as:

$\mathrm{F} 1 \mathrm{M}=((2 * \mathrm{PPV} * \mathrm{TPR}) /(\mathrm{PPV}+\mathrm{TPR}))$

The F1M statistic represented a restricted case of the FM statistic. The F1M statistic was equivalent to the traditional FM statistic. The F1M statistic was equivalent to the balanced FM statistic (Lewis and Gale 1994).

\subsection{Comparative Accuracy Performance Analysis}

\subsubsection{Statistical Procedure}

The Significance Analysis of Microarrays (SAM) algorithm and the Differential Gene Expression (DGE) algorithm were subjected to comparative accuracy performance analysis. The comparative accuracy performance analysis was based on the analysis of microarray datasets in which the numbers and the identities of the differentiallyexpressed genes were known in advance. Microarray datasets containing known differentially-expressed genes do not exist. Therefore, it was necessary to use the synthetic microarray datasets method to generate the synthetic microarray datasets containing known differentially-expressed genes. The synthetic microarray datasets underwent artificial generation, log transformation, Cartesian coordinate calculation and normalization. The comparative accuracy performance analysis consisted of eight procedures:

1. The Gene Expression Omnibus (GEO) repository at the National Center for Biotechnology Information (NCBI) archived and distributed high-throughput molecular abundance data. The high-throughput molecular abundance data consisted primarily of gene expression data generated by microarray technologies. The GEO database has emerged as the leading compendium for the dissemination of gene expression data (Edgar et al. 2002; Barrett et al. 2005; Barrett and Edgar 2006a, 2006b; Barrett et al. 2007). The 
GSE3158 series of murine retinoblastoma tumor microarray datasets was retrieved from the GEO database (Black et al. 2003; Huang et al. 2003). The GSE3158 series of murine retinoblastoma tumor microarray datasets included six murine wild-type tissue microarray datasets: 1. GSM70688, 2. GSM70689, 3. GSM70690, 4. GSM70691, 5. GSM70692 and 6. GSM70693. The single-condition experiment corresponded to six replicated microarray datasets.

2. The single-condition experiment corresponding to six replicated microarray datasets was divided into two states: 1 . control state and 2. experimental state. The control state consisted of three replicated microarray datasets: 1. GSM70688, 2. GSM70689 and 3. GSM70690. The experimental state consisted of three replicated microarray datasets: 1 . GSM70691, 2. GSM70692 and 3. GSM70693. The two-condition experiment was composed of two states in which each state corresponded to three replicated microarray datasets. The two-condition experiment did not contain any known differentiallyexpressed genes.

3. The DGE algorithm was used to compute the 0.01 quantile and the 0.99 quantile. Refer to the synthetic microarray datasets method.

4. A 0.05 normalized fraction of the genes was randomly chosen for upregulation. The 0.05 normalized fraction corresponded to the 330 genes that were randomly selected to become the candidate upregulated differentially-expressed genes. A 0.05 normalized fraction of the genes was randomly chosen for downregulation. The 0.05 normalized fraction corresponded to the 330 genes that were randomly selected to become the candidate downregulated differentially-expressed genes. Refer to the synthetic microarray datasets method.

5. The candidate upregulated differentially-expressed genes were randomly translated by 1.3 to 2.5 times the y magnitude above the 0.99 quantile. The candidate downregulated differentially-expressed genes were randomly translated by 1.3 to 2.5 factors below the 0.01 quantile. Random translation of the candidate upregulated differentially-expressed 
genes and the candidate downregulated differentially-expressed genes generated the synthetic microarray datasets containing known differentially-expressed genes. The twocondition experiment now contained known differentially-expressed genes. Refer to the synthetic microarray datasets method.

6. The SAM algorithm and the DGE algorithm were applied to the synthetic microarray datasets in which the numbers and the identities of the differentially-expressed genes were by construction now known in advance. The SAM algorithm and the DGE algorithm were used to detect the known differentially-expressed genes. The detection rates for the known differentially-expressed genes were analyzed using four methods: 1 . fixed array method, 2. fixed quantile method, 3. differential maximum exclusion method and 4. differential maximum inclusion method.

The fixed array method accuracy performance analysis was used by the SAM algorithm and the DGE algorithm. The fixed array method accuracy performance analysis was based on the examination of a predetermined number of array elements which corresponded to the known number of differentially-regulated genes. The examination of a predetermined number of array elements formed the uniform basis for the comparison of the SAM algorithm accuracy and the DGE algorithm accuracy. The SAM algorithm fixed array method accuracy performance analysis retrieved the last 660 elements from the differential array for the detection of both the downregulated genes and the upregulated genes. The DGE algorithm fixed array method accuracy performance analysis retrieved the first 330 elements from the quantile array for the detection of the downregulated genes. The DGE algorithm fixed array method accuracy performance analysis retrieved the last 330 elements from the quantile array for the detection of the upregulated genes.

The fixed quantile method accuracy performance analysis was used by the DGE algorithm. The DGE algorithm fixed quantile method accuracy performance analysis retrieved a variable number of the quantile array elements below the 0.05 quantile for the detection of the downregulated genes. The DGE algorithm fixed quantile method 
accuracy performance analysis retrieved a variable number of the quantile array elements above the 0.95 quantile for the detection of the upregulated genes. The DGE algorithm fixed quantile method accuracy performance analysis retrieved a variable number of the quantile array elements. The DGE algorithm fixed array method accuracy performance analysis retrieved a fixed number of the quantile array elements. Thus, the DGE algorithm fixed quantile method accuracy performance analysis was different from the DGE algorithm fixed array method accuracy performance analysis.

The differential maximum exclusion method accuracy performance analysis was used by the DGE algorithm. The DGE algorithm differential maximum exclusion method accuracy performance analysis retrieved the quantile array elements below the maximum positive differential quantile $\mathrm{q}_{\mathrm{mp}}$ for the detection of the downregulated genes. The DGE algorithm differential maximum exclusion method accuracy performance analysis retrieved the quantile array elements above the maximum negative differential quantile $\mathrm{q}_{\mathrm{mn}}$ for the detection of the upregulated genes.

The differential maximum inclusion method accuracy performance analysis was used by the DGE algorithm. The DGE algorithm differential maximum inclusion method accuracy performance analysis retrieved the quantile array elements at and below the maximum positive differential quantile $q_{m p}$ for the detection of the downregulated genes. The DGE algorithm differential maximum inclusion method accuracy performance analysis retrieved the quantile array elements at and above the maximum negative differential quantile $\mathrm{q}_{\mathrm{mn}}$ for the detection of the upregulated genes. The DGE algorithm differential maximum inclusion method accuracy performance analysis retrieved the quantile array elements at the maximum positive differential quantile $q_{m p}$ and at the maximum negative differential quantile $\mathrm{q}_{\mathrm{mn}}$. The DGE algorithm differential maximum exclusion method accuracy performance analysis did not retrieve the quantile array elements at the maximum positive differential quantile $q_{m p}$ and at the maximum negative differential quantile $\mathrm{q}_{\mathrm{mn}}$. Thus, the DGE algorithm differential maximum inclusion method accuracy performance analysis was different from the DGE algorithm differential maximum exclusion method accuracy performance analysis. 
7. The comparative accuracy performance analysis consisted of twenty repeated experiments.

8. The experimental results were summarized.

\subsubsection{Microarray Datasets}

The synthetic microarray datasets each contained 330 known upregulated differentially-expressed genes and 330 known downregulated differentially-expressed genes which formed a total of 660 known differentially-expressed genes. The synthetic microarray datasets each contained 660 known differentially-expressed genes and 5920 known nondifferentially-expressed genes which formed a total of 6580 genes. 


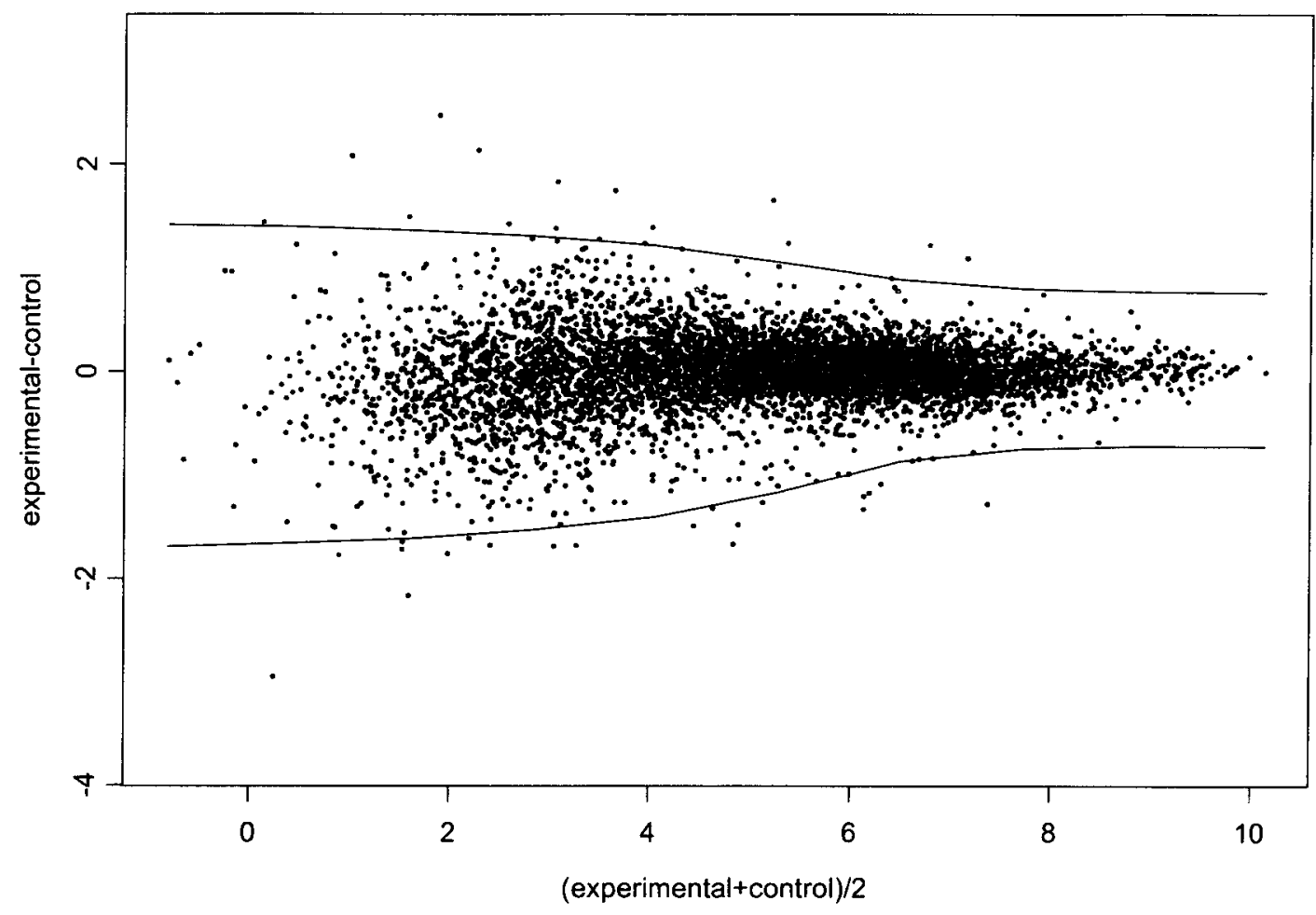

Figure 12. Differential Gene Expression (DGE) algorithm graph including the nonlinear regression 0.01 quantile graph and the nonlinear regression 0.99 quantile graph. The 0.05 normalized fraction genes to be upregulated were represented as the green datapoints. The 0.90 normalized fraction neutral genes were represented as the black datapoints. The 0.05 normalized fraction genes to be downregulated were represented as the red datapoints. 


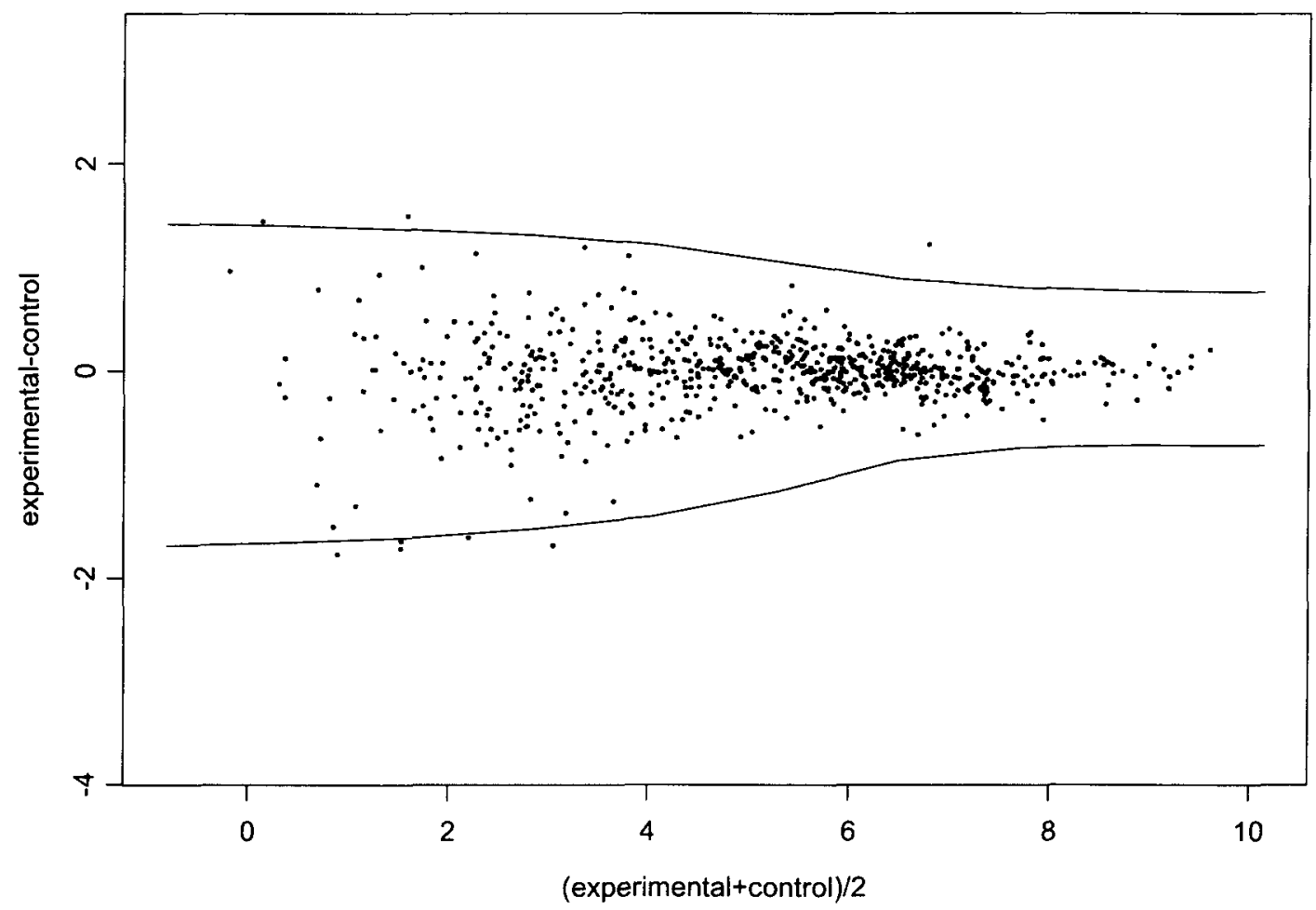

Figure 13. Differential Gene Expression (DGE) algorithm graph including the nonlinear regression 0.01 quantile graph and the nonlinear regression 0.99 quantile graph. The 0.05 normalized fraction genes to be upregulated were represented as the green datapoints. The 0.05 normalized fraction genes to be downregulated were represented as the red datapoints. 


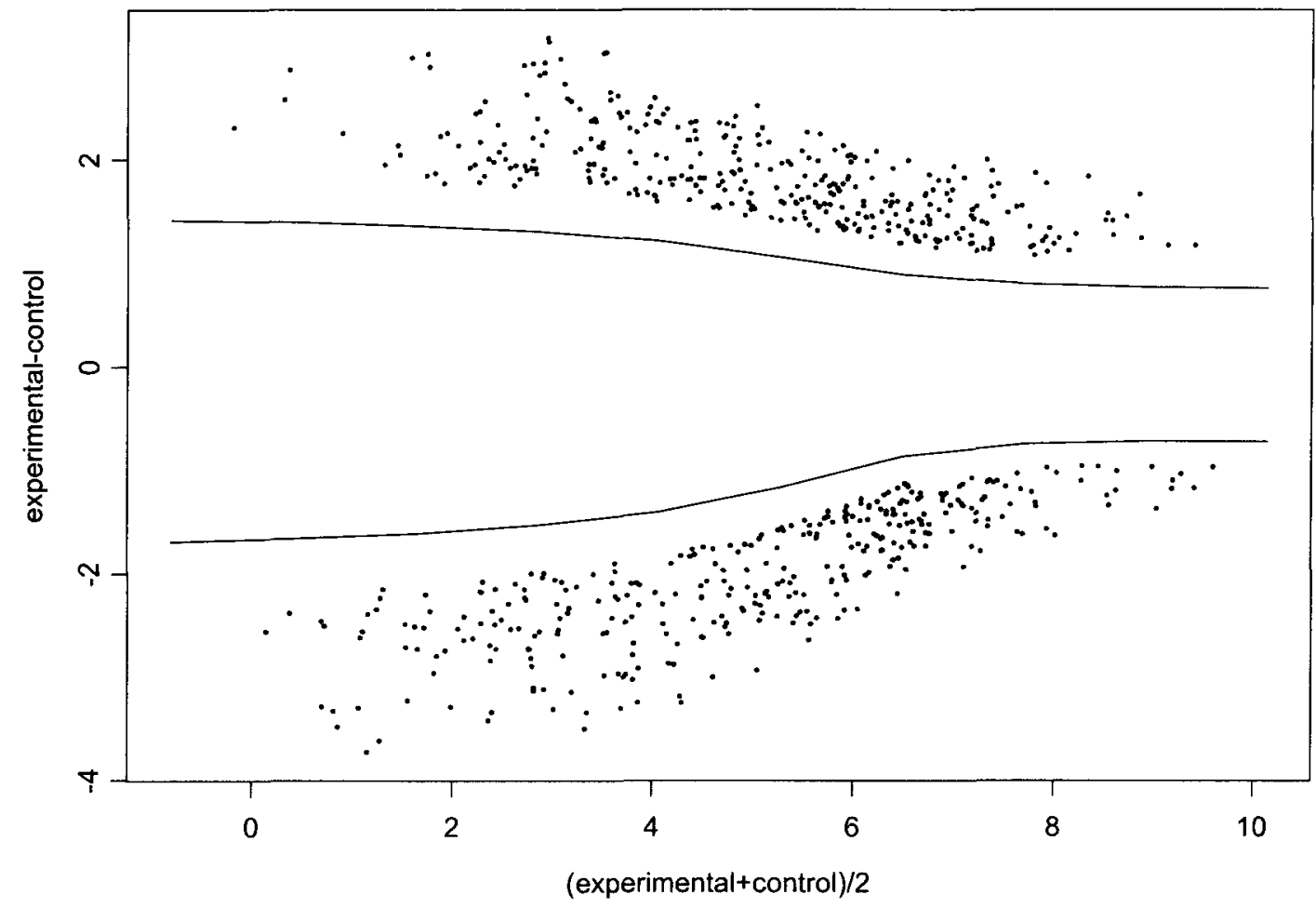

Figure 14. Differential Gene Expression (DGE) algorithm graph including the nonlinear regression 0.01 quantile graph and the nonlinear regression 0.99 quantile graph. The 0.05 normalized fraction upregulated genes were represented as the green datapoints. The 0.05 normalized fraction downregulated genes were represented as the red datapoints. 


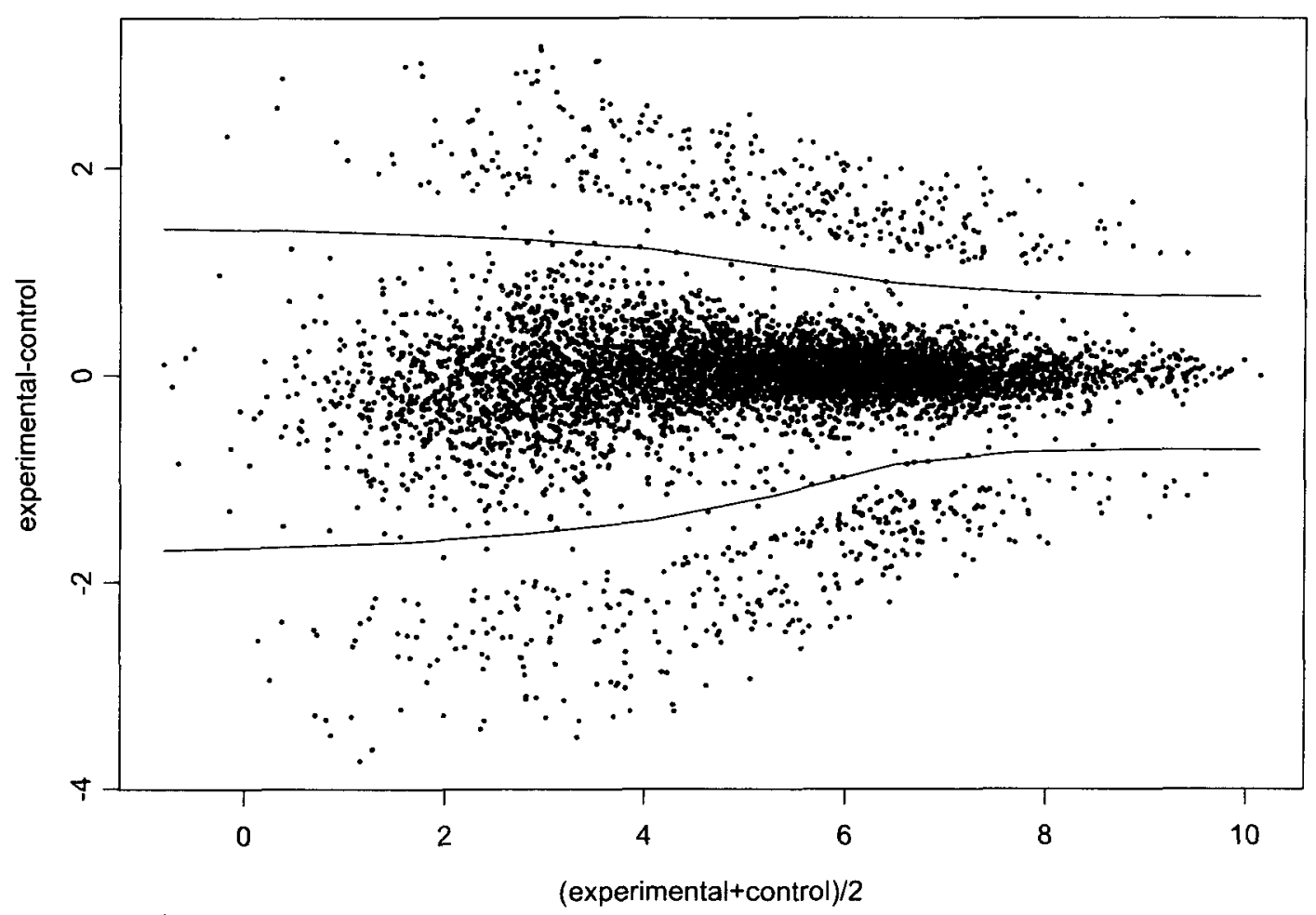

Figure 15. Differential Gene Expression (DGE) algorithm graph including the nonlinear regression 0.01 quantile graph and the nonlinear regression 0.99 quantile graph. The 0.05 normalized fraction upregulated genes were represented as the green datapoints. The 0.90 normalized fraction neutral genes were represented as the black datapoints. The 0.05 normalized fraction downregulated genes were represented as the red datapoints. 


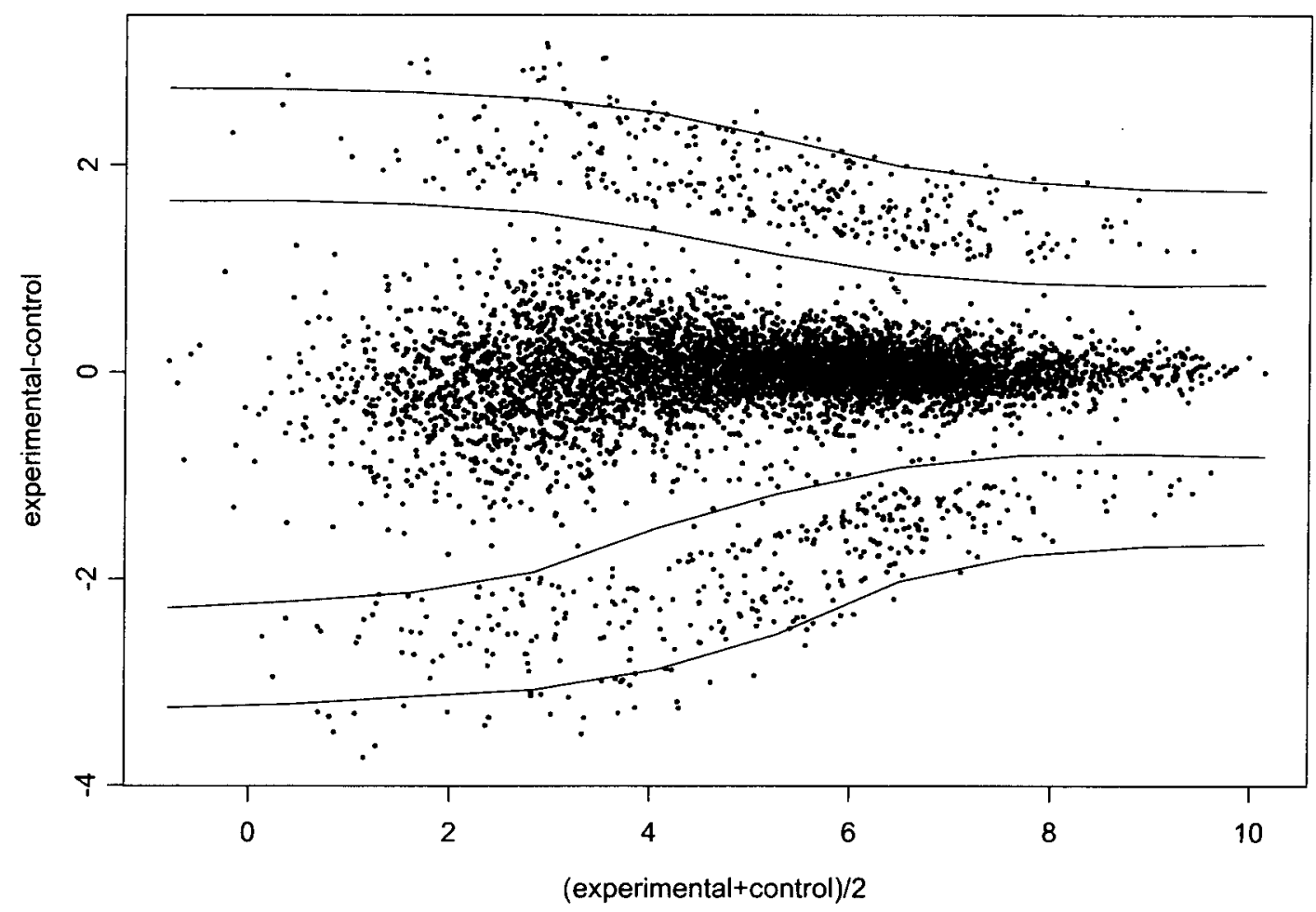

Figure 16. Differential Gene Expression (DGE) algorithm graph including the nonlinear regression 0.01 quantile graph, the nonlinear regression 0.05 quantile graph, the nonlinear regression 0.95 quantile graph and the nonlinear regression 0.99 quantile graph. The 0.05 normalized fraction upregulated genes were represented as the green datapoints. The 0.90 normalized fraction neutral genes were represented as the black datapoints. The 0.05 normalized fraction downregulated genes were represented as the red datapoints. 


\subsubsection{Significance Analysis of Microarrays (SAM) Algorithm}

The accuracy performance analysis was used for the investigation of the Significance Analysis of Microarrays (SAM) algorithm accuracy. Refer to the SAM algorithm difference statistic graph and expected difference statistic graph (Figure 18). The SAM algorithm exhibited a nonsymmetric crossover point. The SAM algorithm demonstrated a nonsymmetric differential distribution between the lower difference statistics and the higher difference statistics. The lower difference statistics exhibited larger differentials and the higher difference statistics exhibited smaller differentials. 


\section{SAM}

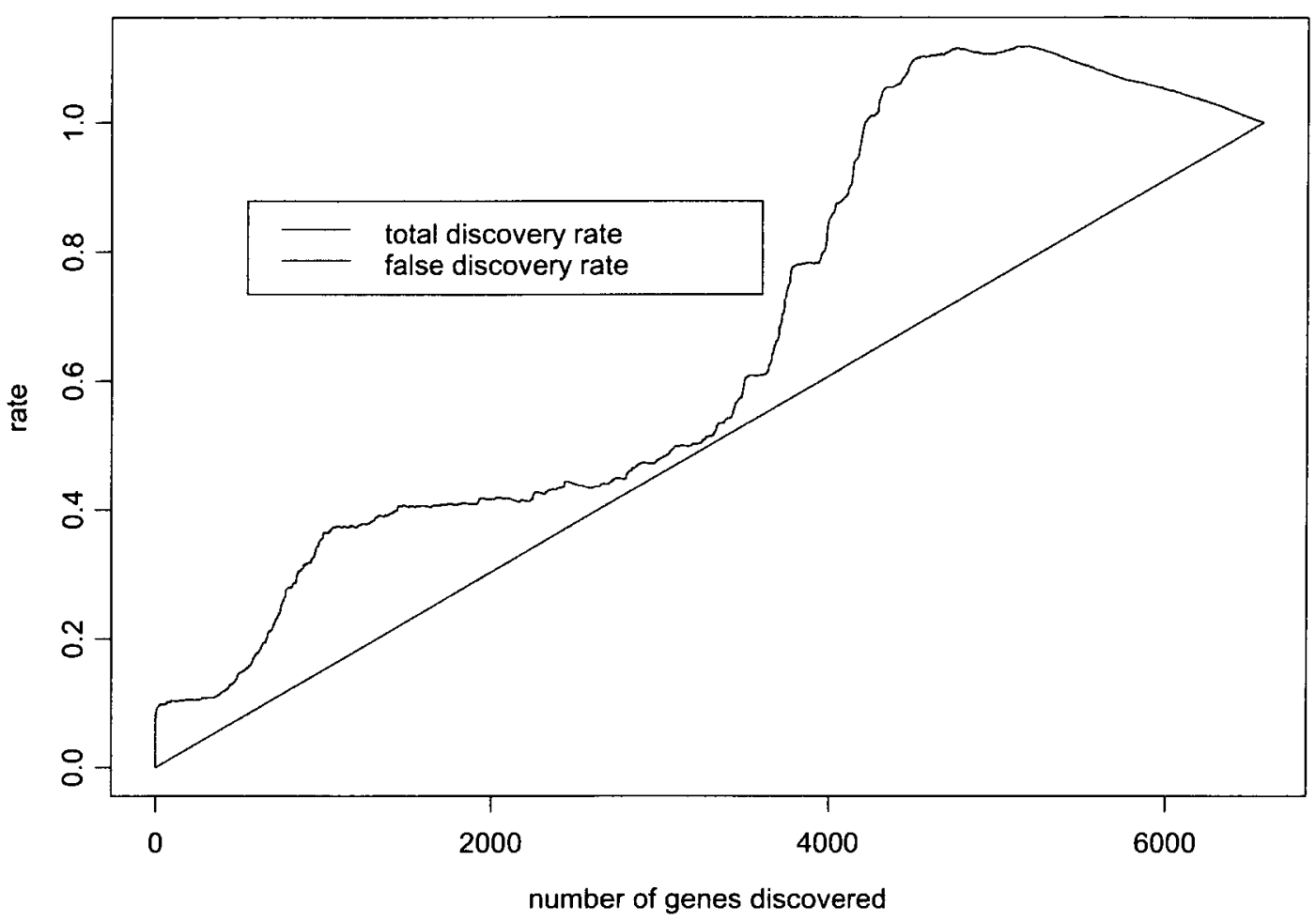

Figure 17. Significance Analysis of Microarrays (SAM) algorithm total discovery rate (TDR) graph and false discovery rate (FDR) graph. The TDR graph was represented as the green line. The FDR graph was represented as the red line. 


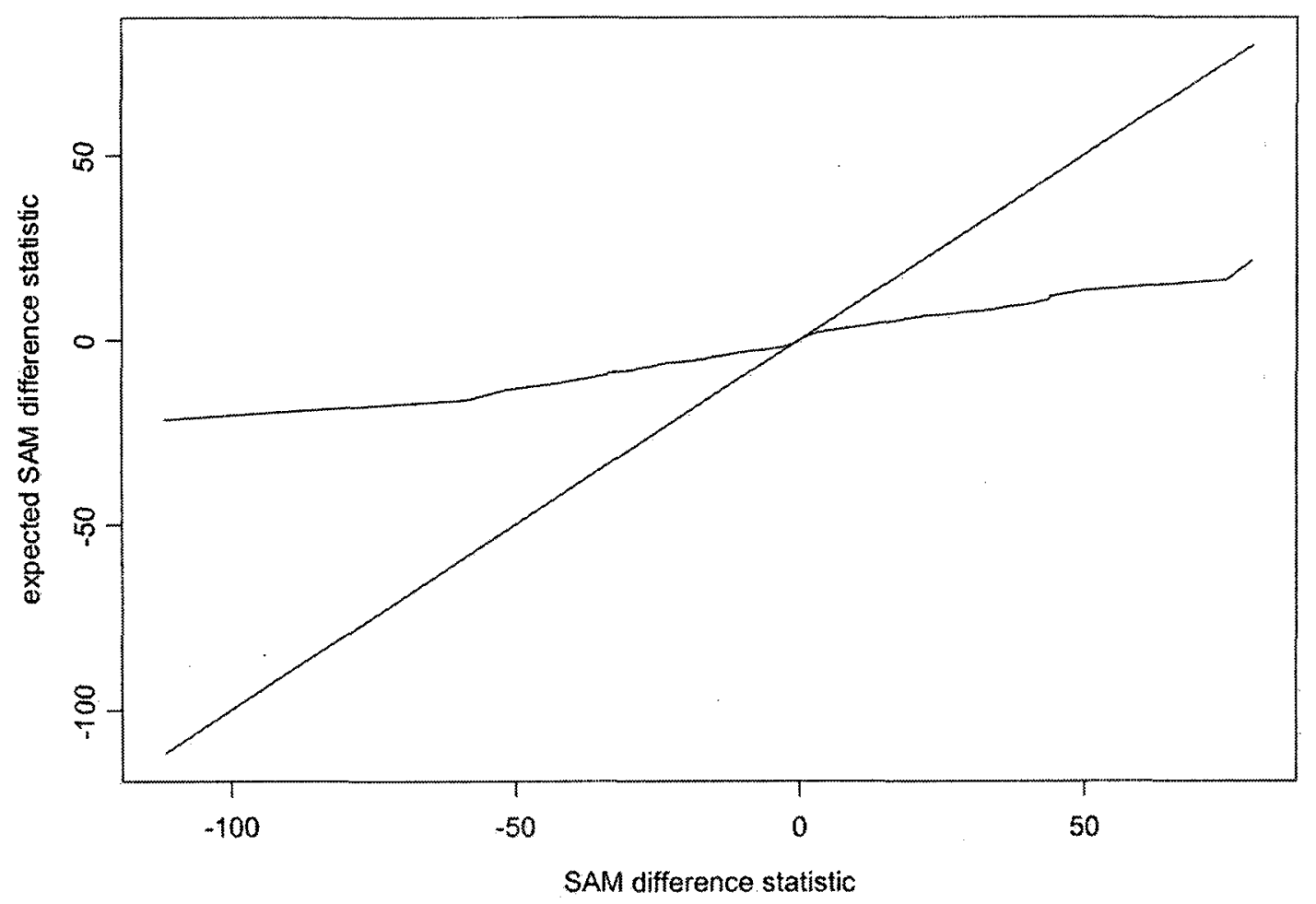

Figure 18. Significance Analysis of Microarrays (SAM) algorithm difference statistic graph and expected difference statistic graph. The nonsymmetric crossover point was represented as the noncentral intersection point between the two intersecting graphs. The nonsymmetric differentials were represented as the nonequal deviations at the extrema between the two intersecting graphs. 


\subsubsection{Significance Analysis of Microarrays (SAM) Fixed Array Method Accuracy Performance Analysis}

The fixed array method accuracy performance analysis was used for the investigation of the Significance Analysis of Microarrays (SAM) algorithm accuracy.

Table 2. Significance Analysis of Microarrays (SAM) algorithm fixed array method accuracy performance analysis.

\begin{tabular}{|c|c|c|c|c|c|c|}
\hline \# & PU & FU & FUP & $\mathrm{PD}$ & $\mathrm{FD}$ & FDP \\
\hline 1 & 330 & 281 & 0.85152 & 330 & 293 & 0.88788 \\
\hline 2 & 330 & 286 & 0.86667 & 330 & 290 & 0.87879 \\
\hline 3 & 330 & 274 & 0.83030 & 330 & 301 & 0.91212 \\
\hline 4 & 330 & 280 & 0.84848 & 330 & 293 & 0.88788 \\
\hline 5 & 330 & 281 & 0.85152 & 330 & 299 & 0.90606 \\
\hline 6 & 330 & 273 & 0.82727 & 330 & 300 & 0.90909 \\
\hline 7 & 330 & 275 & 0.83333 & 330 & 285 & 0.86364 \\
\hline 8 & 330 & 281 & 0.85152 & 330 & 289 & 0.87576 \\
\hline 9 & 330 & 272 & 0.82424 & 330 & 299 & 0.90606 \\
\hline 10 & 330 & 269 & 0.81515 & 330 & 297 & 0.90000 \\
\hline 11 & 330 & 272 & 0.82424 & 330 & 308 & 0.93333 \\
\hline 12 & 330 & 287 & 0.86970 & 330 & 292 & 0.88485 \\
\hline 13 & 330 & 289 & 0.87576 & 330 & 294 & 0.89091 \\
\hline 14 & 330 & 277 & 0.83939 & 330 & 294 & 0.89091 \\
\hline 15 & 330 & 279 & 0.84545 & 330 & 292 & 0.88485 \\
\hline 16 & 330 & 276 & 0.83636 & 330 & 294 & 0.89091 \\
\hline 17 & 330 & 271 & 0.82121 & 330 & 304 & 0.92121 \\
\hline 18 & 330 & 290 & 0.87879 & 330 & 296 & 0.89697 \\
\hline 19 & 330 & 283 & 0.85758 & 330 & 290 & 0.87879 \\
\hline 20 & 330 & 287 & 0.86970 & 330 & 296 & 0.89697 \\
\hline
\end{tabular}

Experiment Number (\#)

Predicted Up (PU)

Found Up (FU)

Found Up Proportion (FUP)

Predicted Down (PD)

Found Down (FD)

Found Down Proportion (FDP) 
Refer to the SAM algorithm fixed array method accuracy performance analysis (Table 2). The SAM algorithm predicted 330 upregulated differentially-expressed genes. The SAM algorithm correctly identified 269 to 290 of the 330 known upregulated differentially-expressed genes. The SAM algorithm correctly identified 0.81515 to 0.87879 normalized fraction of the 330 known upregulated differentially-expressed genes. The SAM algorithm predicted 330 downregulated differentially-expressed genes. The SAM algorithm correctly identified 285 to 308 of the 330 known downregulated differentially-expressed genes. The SAM algorithm correctly identified 0.86364 to 0.93333 normalized fraction of the 330 known downregulated differentially-expressed genes. The SAM algorithm correctly identified a lesser number of the 330 known upregulated differentially-expressed genes and a greater number of 330 known downregulated differentially-expressed genes.

\subsubsection{Differential Gene Expression (DGE) Algorithm}

The accuracy performance analysis was used for the investigation of the Differential Gene Expression (DGE) algorithm accuracy. 


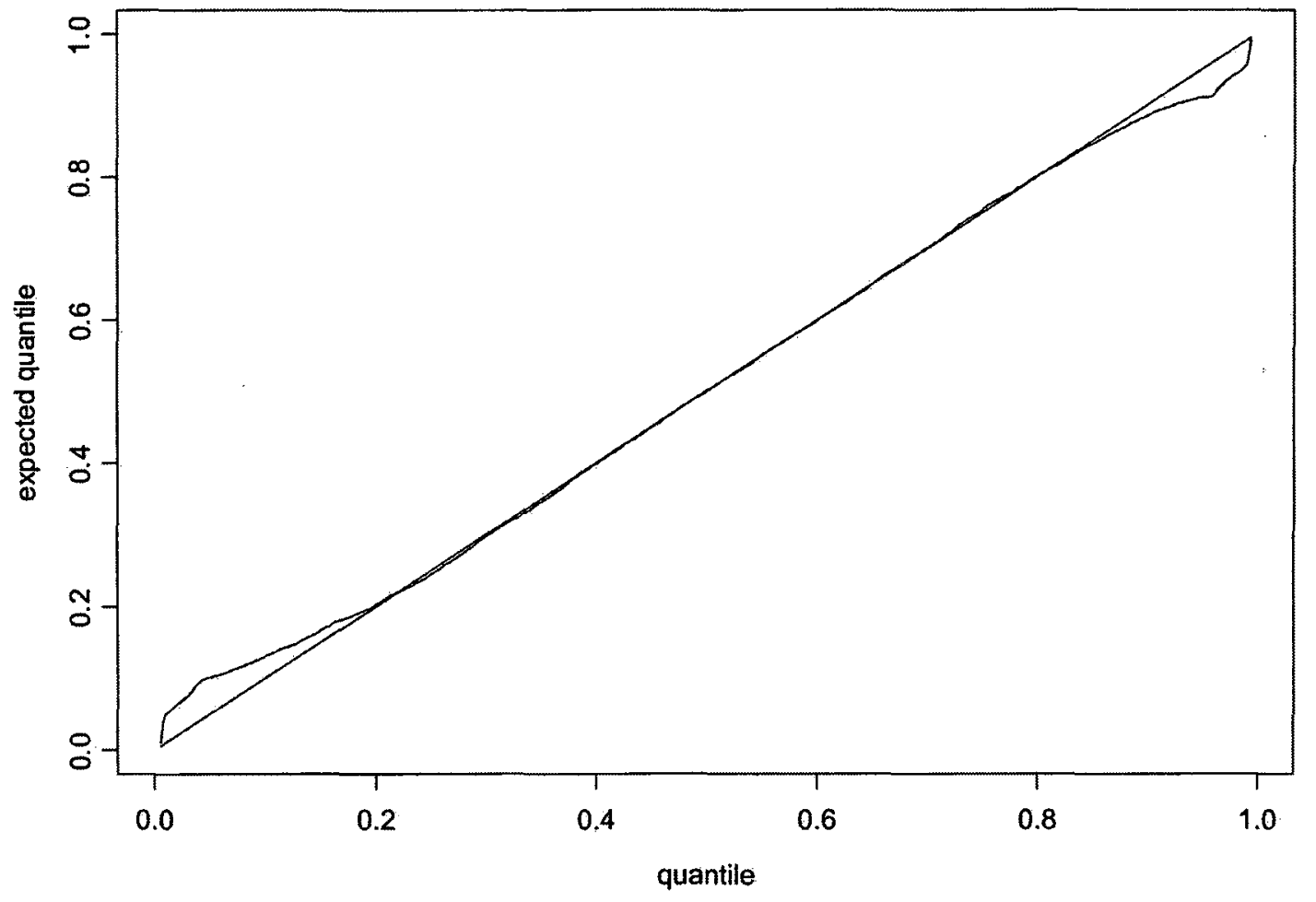

Figure 19. Differential Gene Expression (DGE) algorithm quantile graph and expected quantile graph. The quantile graph was represented as the straight line. The expected quantile graph was represented as the curved line. 


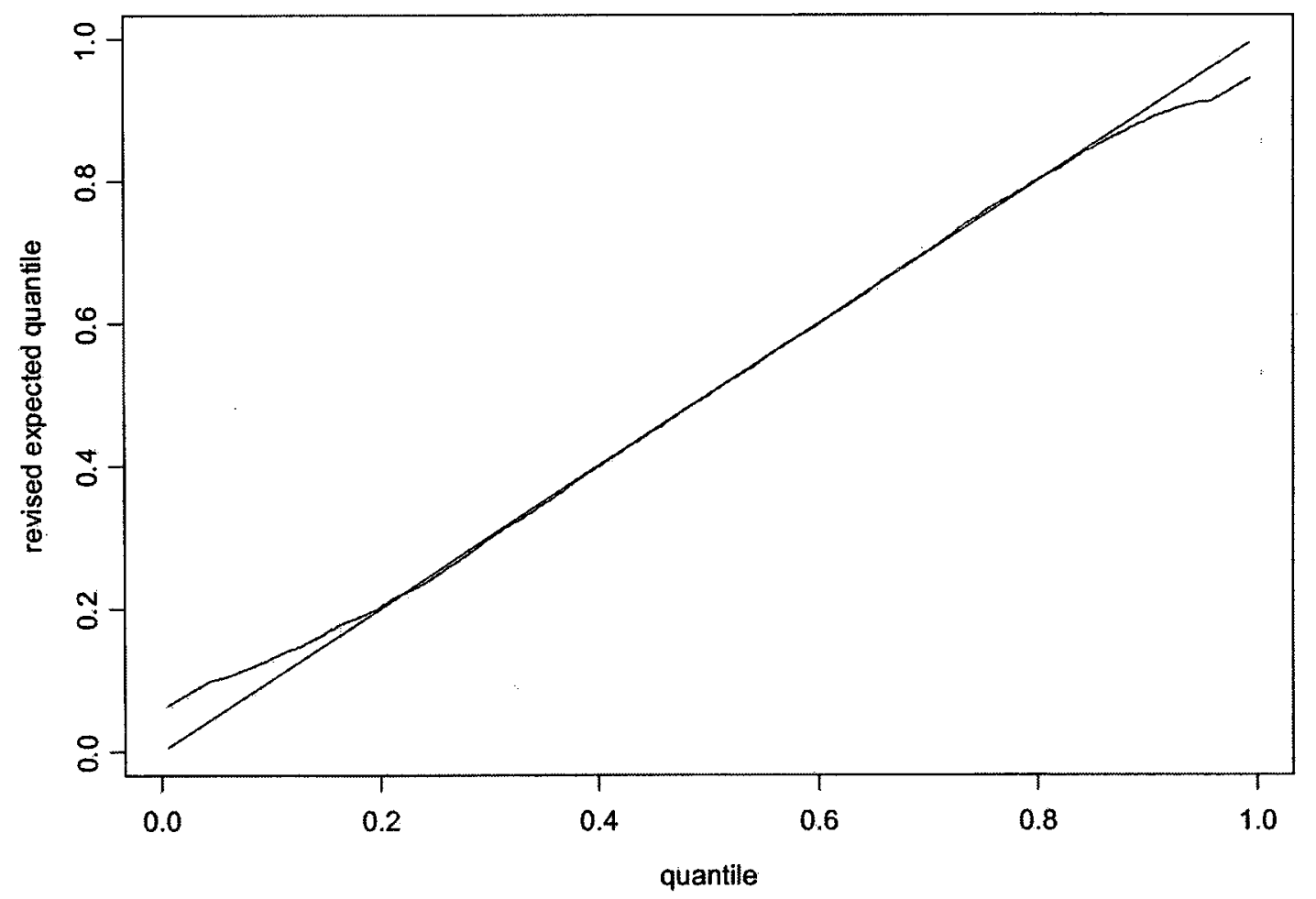

Figure 20. Differential Gene Expression (DGE) algorithm quantile graph and revised expected quantile graph. The quantile graph was represented as the straight line. The revised expected quantile graph was represented as the curved line. 
Refer to the DGE algorithm quantile graph and expected quantile graph (Figure 19). Refer to the DGE algorithm quantile graph and revised expected quantile graph (Figure 20). The DGE algorithm exhibited an approximately symmetric crossover point. The DGE algorithm demonstrated an approximately symmetric differential distribution between the lower quantiles and the higher quantiles. The lower quantiles and the higher quantiles exhibited approximately equal differentials.

\subsubsection{Differential Gene Expression (DGE) Fixed Array Method Accuracy Performance Analysis}

The fixed array method accuracy performance analysis was used for the investigation of the Differential Gene Expression (DGE) algorithm accuracy.

Table 3. Differential Gene Expression (DGE) algorithm fixed array method accuracy performance analysis.

$\begin{array}{rllllll}\# & \text { PU } & \text { FU } & \text { FUP } & \text { PD } & \text { FD } & \text { FDP } \\ 1 & 330 & 324 & 0.98182 & 330 & 322 & 0.97576 \\ 2 & 330 & 323 & 0.97879 & 330 & 325 & 0.98485 \\ 3 & 330 & 323 & 0.97879 & 330 & 326 & 0.98788 \\ 4 & 330 & 324 & 0.98182 & 330 & 325 & 0.98485 \\ 5 & 330 & 324 & 0.98182 & 330 & 324 & 0.98182 \\ 6 & 330 & 324 & 0.98182 & 330 & 325 & 0.98485 \\ 7 & 330 & 323 & 0.97879 & 330 & 325 & 0.98485 \\ 8 & 330 & 324 & 0.98182 & 330 & 325 & 0.98485 \\ 9 & 330 & 324 & 0.98182 & 330 & 323 & 0.97879 \\ 10 & 330 & 324 & 0.98182 & 330 & 323 & 0.97879 \\ 11 & 330 & 323 & 0.97879 & 330 & 326 & 0.98788 \\ 12 & 330 & 323 & 0.97879 & 330 & 325 & 0.98485 \\ 13 & 330 & 324 & 0.98182 & 330 & 325 & 0.98485 \\ 14 & 330 & 324 & 0.98182 & 330 & 326 & 0.98788 \\ 15 & 330 & 323 & 0.97879 & 330 & 325 & 0.98485 \\ 16 & 330 & 323 & 0.97879 & 330 & 325 & 0.98485 \\ 17 & 330 & 324 & 0.98182 & 330 & 326 & 0.98788 \\ 18 & 330 & 324 & 0.98182 & 330 & 325 & 0.98485 \\ 19 & 330 & 326 & 0.98788 & 330 & 323 & 0.97879 \\ 20 & 330 & 323 & 0.97879 & 330 & 324 & 0.98182\end{array}$


Experiment Number (\#)

Predicted Up (PU)

Found Up (FU)

Found Up Proportion (FUP)

Predicted Down (PD)

Found Down (FD)

Found Down Proportion (FDP)

Refer to the DGE algorithm fixed array method accuracy performance analysis (Table 3). The DGE algorithm predicted 330 upregulated differentially-expressed genes. The DGE algorithm correctly identified 323 to 326 of the 330 known upregulated differentially-expressed genes. The DGE algorithm correctly identified 0.97879 to 0.98788 normalized fraction of the 330 known upregulated differentially-expressed genes. The DGE algorithm predicted 330 downregulated differentially-expressed genes. The DGE algorithm correctly identified 322 to 326 of the 330 known downregulated differentially-expressed genes. The DGE algorithm correctly identified 0.97576 to 0.98788 normalized fraction of the 330 known downregulated differentially-expressed genes. The DGE algorithm correctly identified an approximately equal number of the 330 known upregulated differentially-expressed genes and the 330 known downregulated differentially-expressed genes.

The DGE algorithm correctly identified 34 to 55 more of the 330 known upregulated differentially-expressed genes than the Significance Analysis of Microarrays (SAM) algorithm. The DGE algorithm was more accurate by 0.10303 to 0.16667 normalized fraction for the 330 known upregulated differentially-expressed genes than the SAM algorithm. The DGE algorithm correctly identified 18 to 40 more of the 330 known downregulated differentially-expressed genes than the SAM algorithm. The DGE algorithm was more accurate by 0.05454 to 0.12121 normalized fraction for the 330 known downregulated differentially-expressed genes than the SAM algorithm.

The fixed array method accuracy performance analysis demonstrated that the DGE algorithm was the more accurate method than the SAM algorithm. 


\subsubsection{Differential Gene Expression (DGE) Fixed Quantile Method Accuracy Performance Analysis}

The fixed quantile method accuracy performance analysis was used for the investigation of the Differential Gene Expresion (DGE) algorithm accuracy.

Table 4. Differential Gene Expression (DGE) algorithm fixed quantile method accuracy performance analysis.

\begin{tabular}{|c|c|c|c|c|c|c|}
\hline$\#$ & PU & FU & FUP & $\mathrm{PD}$ & FD & FDP \\
\hline 1 & 339 & 330 & 1.00000 & 340 & 329 & 0.99697 \\
\hline 2 & 338 & 330 & 1.00000 & 341 & 330 & 1.00000 \\
\hline 3 & 340 & 330 & 1.00000 & 340 & 330 & 1.00000 \\
\hline 4 & 338 & 330 & 1.00000 & 340 & 330 & 1.00000 \\
\hline 5 & 339 & 330 & 1.00000 & 344 & 330 & 1.00000 \\
\hline 6 & 339 & 330 & 1.00000 & 340 & 330 & 1.00000 \\
\hline 7 & 339 & 330 & 1.00000 & 341 & 330 & 1.00000 \\
\hline 8 & 339 & 330 & 1.00000 & 339 & 330 & 1.00000 \\
\hline 9 & 339 & 330 & 1.00000 & 343 & 330 & 1.00000 \\
\hline 10 & 338 & 330 & 1.00000 & 341 & 330 & 1.00000 \\
\hline 11 & 340 & 330 & 1.00000 & 339 & 330 & 1.00000 \\
\hline 12 & 339 & 330 & 1.00000 & 340 & 330 & 1.00000 \\
\hline 13 & 341 & 330 & 1.00000 & 340 & 330 & 1.00000 \\
\hline 14 & 339 & 330 & 1.00000 & 340 & 330 & 1.00000 \\
\hline 15 & 340 & 330 & 1.00000 & 340 & 330 & 1.00000 \\
\hline 16 & 338 & 330 & 1.00000 & 342 & 330 & 1.00000 \\
\hline 17 & 340 & 330 & 1.00000 & 339 & 330 & 1.00000 \\
\hline 18 & 342 & 330 & 1.00000 & 339 & 330 & 1.00000 \\
\hline 19 & 336 & 330 & 1.00000 & 342 & 330 & 1.00000 \\
\hline 20 & 340 & 330 & 1.00000 & 346 & 330 & 1.00000 \\
\hline
\end{tabular}

Experiment Number (\#)

Predicted Up (PU)

Found Up (FU)

Found Up Proportion (FUP)

Predicted Down (PD)

Found Down (FD)

Found Down Proportion (FDP) 
Refer to the DGE algorithm fixed quantile method accuracy performance analysis (Table 4). The DGE algorithm predicted 336 to 342 upregulated differentially-expressed genes. The DGE algorithm correctly identified 330 of the 330 known upregulated differentially-expressed genes. The DGE algorithm correctly identified 1.00000 normalized fraction of the 330 known upregulated differentially-expressed genes. The DGE algorithm predicted 339 to 346 downregulated differentially-expressed genes. The DGE algorithm correctly identified 329 to 330 of the 330 known downregulated differentially-expressed genes. The DGE algorithm correctly identified 0.99697 to 1.00000 normalized fraction of the 330 known downregulated differentially-expressed genes. The DGE algorithm correctly identified an approximately equal number of the 330 known upregulated differentially-expressed genes and the 330 known downregulated differentially-expressed genes.

The DGE algorithm incorrectly identified 6 to 12 of the 5920 known nondifferentially-expressed genes as upregulated genes. The DGE algorithm incorrectly identified 9 to 16 of the 5920 known nondifferentially-expressed genes as downregulated genes. The DGE algorithm incorrectly identified 18 to 26 of the 5920 known nondifferentially-expressed genes as differentially-expressed genes. The large number of nondifferentially-expressed genes that were misclassified as differentially-expressed genes demonstrated that the fixed quantile method accurary performance analysis was not suitable for further consideration.

\subsubsection{Differential Gene Expression (DGE) Differential Maximum Exclusion Method Accuracy Performance Analysis}

The differential maximum exclusion method accuracy performance analysis was used for the investigation of the Differential Gene Expression (DGE) algorithm accuracy.

Table 5. Differential Gene Expression (DGE) algorithm differential maximum exclusion method accuracy performance analysis. 


\# $\quad$ PU FU FUP $\quad$ PD FD FDP

$\begin{array}{rllllll}1 & 331 & 325 & 0.98485 & 325 & 318 & 0.96364 \\ 2 & 319 & 312 & 0.94545 & 332 & 327 & 0.99091 \\ 3 & 325 & 318 & 0.96364 & 332 & 328 & 0.99394 \\ 4 & 333 & 327 & 0.99091 & 337 & 329 & 0.99697 \\ 5 & 312 & 307 & 0.93030 & 320 & 315 & 0.95455 \\ 6 & 331 & 325 & 0.98485 & 328 & 323 & 0.97879 \\ 7 & 322 & 315 & 0.95455 & 332 & 327 & 0.99091 \\ 8 & 335 & 328 & 0.99394 & 328 & 324 & 0.98182 \\ 9 & 329 & 323 & 0.97879 & 332 & 325 & 0.98485 \\ 10 & 334 & 327 & 0.99091 & 296 & 292 & 0.88485 \\ 11 & 333 & 326 & 0.98788 & 325 & 322 & 0.97576 \\ 12 & 320 & 314 & 0.95152 & 322 & 318 & 0.96364 \\ 13 & 326 & 320 & 0.96970 & 333 & 327 & 0.99091 \\ 14 & 330 & 324 & 0.98182 & 321 & 317 & 0.96061 \\ 15 & 333 & 325 & 0.98485 & 324 & 320 & 0.96970 \\ 16 & 336 & 328 & 0.99394 & 328 & 323 & 0.97879 \\ 17 & 319 & 313 & 0.94848 & 328 & 324 & 0.98182 \\ 18 & 318 & 312 & 0.94545 & 323 & 319 & 0.96667 \\ 19 & 324 & 320 & 0.96970 & 318 & 313 & 0.94848 \\ 20 & 321 & 314 & 0.95152 & 312 & 307 & 0.93030\end{array}$

Experiment Number (\#)

Predicted Up (PU)

Found Up (FU)

Found Up Proportion (FUP)

Predicted Down (PD)

Found Down (FD)

Found Down Proportion (FDP)

Refer to the DGE algorithm differential maximum exclusion method accuracy performance analysis (Table 5). The DGE algorithm predicted 312 to 336 upregulated differentially-expressed genes. The DGE algorithm correctly identified 307 to 328 of the 330 known upregulated differentially-expressed genes. The DGE algorithm correctly identified 0.93030 to 0.99394 normalized fraction of the 330 known upregulated differentially-expressed genes. The DGE algorithm predicted 296 to 337 downregulated differentially-expressed genes. The DGE algorithm correctly identified 292 to 329 of the 330 known downregulated differentially-expressed genes. The DGE algorithm correctly 
identified 0.88485 to 0.99697 normalized fraction of the 330 known downregulated differentially-expressed genes. The DGE algorithm correctly identified an approximately equal number of the 330 known upregulated differentially-expressed genes and the 330 known downregulated differentially-expressed genes.

The fixed array method accuracy performance analysis and the fixed quantile method accuracy performance analysis required advance knowledge of the number of differentially-expressed genes in the microarray datasets. The fixed array method accuracy performance analysis used two predetermined numbers of array elements for the detection of the differentially-expressed genes. The fixed quantile method accuracy performance analysis used two predetermined quantiles for the detection of the differentially-expressed genes. The differential maximum exclusion method accuracy performance analysis did not require advance knowledge of the number of differentiallyexpressed genes in the microarray datasets. The differential maximum exclusion method accuracy performance analysis used two automatically-determined quantiles for the detection of the differentially-expressed genes. The differential maximum exclusion method accuracy performance analysis was the more accurate method than the fixed array method accuracy performance analysis and the fixed quantile method accuracy performance analysis.

\subsubsection{Differential Gene Expression (DGE) Differential Maximum Inclusion Method Accuracy Performance Analysis}

The differential maximum inclusion method accuracy performance analysis was used for the investigation of the Differential Gene Expression (DGE) algorithm accuracy.

Table 6. Differential Gene Expression (DGE) algorithm differential maximum inclusion method accuracy performance analysis.

\# PU FU FUP $\quad$ PD FD FDP 


$\begin{array}{rllllll}1 & 332 & 326 & 0.98788 & 326 & 319 & 0.96667 \\ 2 & 320 & 313 & 0.94848 & 333 & 328 & 0.99394 \\ 3 & 326 & 319 & 0.96667 & 333 & 329 & 0.99697 \\ 4 & 334 & 328 & 0.99394 & 338 & 330 & 1.00000 \\ 5 & 313 & 308 & 0.93333 & 321 & 316 & 0.95758 \\ 6 & 332 & 326 & 0.98788 & 329 & 324 & 0.98182 \\ 7 & 323 & 316 & 0.95758 & 333 & 328 & 0.99394 \\ 8 & 336 & 329 & 0.99697 & 329 & 325 & 0.98485 \\ 9 & 330 & 324 & 0.98182 & 333 & 325 & 0.98485 \\ 10 & 335 & 328 & 0.99394 & 297 & 293 & 0.88788 \\ 11 & 334 & 327 & 0.99091 & 326 & 323 & 0.97879 \\ 12 & 321 & 315 & 0.95455 & 323 & 319 & 0.96667 \\ 13 & 327 & 321 & 0.97273 & 334 & 328 & 0.99394 \\ 14 & 331 & 325 & 0.98485 & 322 & 318 & 0.96364 \\ 15 & 334 & 326 & 0.98788 & 325 & 321 & 0.97273 \\ 16 & 337 & 329 & 0.99697 & 329 & 324 & 0.98182 \\ 17 & 320 & 314 & 0.95152 & 329 & 325 & 0.98485 \\ 18 & 319 & 313 & 0.94848 & 324 & 320 & 0.96970 \\ 19 & 325 & 321 & 0.97273 & 319 & 314 & 0.95152 \\ 20 & 322 & 315 & 0.95455 & 313 & 308 & 0.93333\end{array}$

Experiment Number (\#)

Predicted Up (PU)

Found Up (FU)

Found Up Proportion (FUP)

Predicted Down (PD)

Found Down (FD)

Found Down Proportion (FDP)

Refer to the DGE algorithm differential maximum inclusion method accuracy performance analysis (Table 6). The DGE algorithm predicted 313 to 337 upregulated differentially-expressed genes. The DGE algorithm correctly identified 308 to 329 of the 330 known upregulated differentially-expressed genes. The DGE algorithm correctly identified 0.93333 to 0.99697 normalized fraction of the 330 known upregulated differentially-expressed genes. The DGE algorithm predicted 297 to 338 downregulated differentially-expressed genes. The DGE algorithm correctly identified 293 to 330 of the 330 known downregulated differentially-expressed genes. The DGE algorithm correctly identified 0.88788 to 1.00000 normalized fraction of the 330 known downregulated differentially-expressed genes. The DGE algorithm correctly identified an approximately 
equal number of the 330 known upregulated differentially-expressed genes and the 330 known downregulated differentially-expressed genes.

The differential maximum inclusion method accuracy performance analysis retrieved the quantile array elements at and above the maximum negative differential quantile $\mathrm{q}_{\mathrm{mn}}$ for the detection of the upregulated genes. The differential maximum exclusion method accuracy performance analysis retrieved the quantile array elements above the maximum negative differential quantile $\mathrm{q}_{\mathrm{mn}}$ for the detection of the upregulated genes. The differential maximum inclusion method accuracy performance analysis retrieved the quantile array element at the maximum negative differential quantile $q_{m n}$. The differential maximum exclusion method accuracy performance analysis did not retrieve the quantile array element at the maximum negative differential quantile $\mathrm{q}_{\mathrm{mn}}$. The differential maximum inclusion method accuracy performance analysis retrieved one quantile array element more than the differential maximum exclusion method accuracy performance analysis. In all twenty iterations, the differential maximum inclusion method accuracy performance analysis correctly identified one upregulated gene more than the differential maximum exclusion method accuracy performance analysis.

The differential maximum inclusion method accuracy performance analysis retrieved the quantile array elements at and below the maximum positive differential quantile $\mathrm{q}_{\mathrm{mp}}$ for the detection of the downregulated genes. The differential maximum exclusion method accuracy performance analysis retrieved the quantile array elements below the maximum positive differential quantile $\mathrm{q}_{\mathrm{mp}}$ for the detection of the downregulated genes. The differential maximum inclusion method accuracy performance analysis retrieved the quantile array element at the maximum positive differential quantile $\mathrm{q}_{\mathrm{mp}}$. The differential maximum exclusion method accuracy performance analysis did not retrieve the quantile array element at the maximum positive differential quantile $\mathrm{q}_{\mathrm{mp}}$. The differential maximum inclusion method accuracy performance analysis retrieved one quantile array element more than the differential maximum exclusion method accuracy performance analysis. In the ninth iteration, the differential maximum inclusion method accuracy performance analysis correctly 
identified the same number of downregulated genes as the differential maximum exclusion method accuracy performance analysis. In the remaining nineteen iterations, the differential maximum inclusion method accuracy performance analysis correctly identified one downregulated gene more than the differential maximum exclusion method accuracy performance analysis.

The differential maximum inclusion method accuracy performance analysis was the more accurate method than the differential maximum exclusion method accuracy performance analysis. The differential maximum inclusion method accuracy performance analysis demonstrated that using the differential maximum inclusion method in the DGE algorithm was the most accurate method.

\subsubsection{Differential Gene Expression (DGE) Differential Maximum Inclusion Method Accuracy Performance Analysis Using the Confusion Matrix Classification System}

The differential maximum inclusion method accuracy performance analysis was used for the more detailed investigation of the Differential Gene Expression (DGE) algorithm accuracy. The DGE algorithm differential maximum inclusion method accuracy performance analysis was based on the confusion matrix classification system. The confusion matrix classification performance analysis consisted of fourteen performance statistics: 1. true positive (TP), 2. false positive (FP), 3. true negative (TN), 4. false negative (FN), 5. true positive rate (TPR), 6. false positive rate (FPR), 7. true negative rate (TNR), 8. false negative rate (FNR), 9. positive predictive value (PPV), 10. negative predictive value (NPV), 11. accuracy (AC), 12. geometric-mean $(\mathrm{GM} 1), 13$. geometric-mean $2(\mathrm{GM} 2)$ and 14. $\mathrm{F}_{1}$-measure $(\mathrm{F} 1 \mathrm{M})$.

Table 7a. Differential Gene Expression (DGE) algorithm differential maximum inclusion method accuracy performance analysis using the confusion matrix classification system for the upregulated genes.

$\begin{array}{lllllllll}\text { \# } & \text { TP } & \text { FP } & \text { TN } & \text { FN } & \text { TPR } & \text { FPR } & \text { TNR } & \text { FNR }\end{array}$




$\begin{array}{rrrrrrrrr}1 & 326 & 6 & 6244 & 4 & 0.98788 & 0.00096 & 0.99904 & 0.01212 \\ 2 & 313 & 7 & 6243 & 17 & 0.94848 & 0.00112 & 0.99888 & 0.05152 \\ 3 & 319 & 7 & 6243 & 11 & 0.96667 & 0.00112 & 0.99888 & 0.03333 \\ 4 & 328 & 6 & 6244 & 2 & 0.99394 & 0.00096 & 0.99904 & 0.00606 \\ 5 & 308 & 5 & 6245 & 22 & 0.93333 & 0.00080 & 0.99920 & 0.06667 \\ 6 & 326 & 6 & 6244 & 4 & 0.98788 & 0.00096 & 0.99904 & 0.01212 \\ 7 & 316 & 7 & 6243 & 14 & 0.95758 & 0.00112 & 0.99888 & 0.04242 \\ 8 & 329 & 7 & 6243 & 1 & 0.99697 & 0.00112 & 0.99888 & 0.00303 \\ 9 & 324 & 6 & 6244 & 6 & 0.98182 & 0.00096 & 0.99904 & 0.01818 \\ 10 & 328 & 7 & 6243 & 2 & 0.99394 & 0.00112 & 0.99888 & 0.00606 \\ 11 & 327 & 7 & 6243 & 3 & 0.99091 & 0.00112 & 0.99888 & 0.00909 \\ 12 & 315 & 6 & 6244 & 15 & 0.95455 & 0.00096 & 0.99904 & 0.04545 \\ 13 & 321 & 6 & 6244 & 9 & 0.97273 & 0.00096 & 0.99904 & 0.02727 \\ 14 & 325 & 6 & 6244 & 5 & 0.98485 & 0.00096 & 0.99904 & 0.01515 \\ 15 & 326 & 8 & 6242 & 4 & 0.98788 & 0.00128 & 0.99872 & 0.01212 \\ 16 & 329 & 8 & 6242 & 1 & 0.99697 & 0.00128 & 0.99872 & 0.00303 \\ 17 & 314 & 6 & 6244 & 16 & 0.95152 & 0.00096 & 0.99904 & 0.04848 \\ 18 & 313 & 6 & 6244 & 17 & 0.94848 & 0.00096 & 0.99904 & 0.05152 \\ 19 & 321 & 4 & 6246 & 9 & 0.97273 & 0.00064 & 0.99936 & 0.02727 \\ 20 & 315 & 7 & 6243 & 15 & 0.95455 & 0.00112 & 0.99888 & 0.04545\end{array}$

Experiment Number (\#)

True Positive (TP)

False Positive (FP)

True Negative (TN)

False Negative (FN)

True Positive Rate (TPR)

False Positive Rate (FPR)

True Negative Rate (TNR)

False Negative Rate (FNR)

Table 7b. Differential Gene Expression (DGE) algorithm differential maximum inclusion method accuracy performance analysis using the confusion matrix classification system for the upregulated genes.

\# $\mathrm{NPV} \quad \mathrm{AC} \quad$ GM1 GM2 $\quad$ F1M
$\begin{array}{llll}1 & 0.98193 & 0.99936\end{array}$
$\begin{array}{lllll}0.99848 & 0.98490 & 0.99344 & 0.98489\end{array}$
20.97813
0.99728
0.99635
0.96319
0.97336
0.96308
30.97853
0.99824
0.99726
0.97258
0.98264
0.97256
$\begin{array}{llll}4 & 0.98204 & 0.99968\end{array}$
0.99878
0.98797
0.99649
0.98795
$\begin{array}{lll}5 & 0.98403\end{array}$
0.99649
$\begin{array}{lll}0.99590 & 0.95834\end{array}$
0.96571
0.95801
60.98193
0.99936
0.99848
0.98490
$0.99344 \quad 0.98489$ 


$\begin{array}{rllllll}7 & 0.97833 & 0.99776 & 0.99681 & 0.96790 & 0.97801 & 0.96784 \\ 8 & 0.97917 & 0.99984 & 0.99878 & 0.98803 & 0.99792 & 0.98799 \\ 9 & 0.98182 & 0.99904 & 0.99818 & 0.98182 & 0.99039 & 0.98182 \\ 10 & 0.97910 & 0.99968 & 0.99863 & 0.98649 & 0.99641 & 0.98647 \\ 11 & 0.97904 & 0.99952 & 0.99848 & 0.98496 & 0.99489 & 0.98494 \\ 12 & 0.98131 & 0.99760 & 0.99681 & 0.96783 & 0.97654 & 0.96774 \\ 13 & 0.98165 & 0.99856 & 0.99772 & 0.97718 & 0.98580 & 0.97717 \\ 14 & 0.98187 & 0.99920 & 0.99833 & 0.98336 & 0.99192 & 0.98336 \\ 15 & 0.97605 & 0.99936 & 0.99818 & 0.98195 & 0.99328 & 0.98193 \\ 16 & 0.97626 & 0.99984 & 0.99863 & 0.98656 & 0.99784 & 0.98651 \\ 17 & 0.98125 & 0.99744 & 0.99666 & 0.96627 & 0.97499 & 0.96615 \\ 18 & 0.98119 & 0.99728 & 0.99650 & 0.96470 & 0.97343 & 0.96456 \\ 19 & 0.98769 & 0.99856 & 0.99802 & 0.98018 & 0.98595 & 0.98015 \\ 20 & 0.97826 & 0.99760 & 0.99666 & 0.96633 & 0.97646 & 0.96626\end{array}$

Experiment Number (\#)

Positive Predictive Value (PPV)

Negative Predictive Value (NPV)

Accuracy (AC)

Geometric-Mean $_{1}$ (GM1)

Geometric-Mean 2 (GM2)

$\mathrm{F}_{1}$-Measure (F1M)

Table 8a. Differential Gene Expression (DGE) algorithm differential maximum inclusion method accuracy performance analysis using the confusion matrix classification system for the downregulated genes.

$\begin{array}{lllllllll}\text { \# } & \text { TP } & \text { FP } & \text { TN } & \text { FN } & \text { TPR } & \text { FPR } & \text { TNR } & \text { FNR }\end{array}$

$\begin{array}{rrrrrrrrr}1 & 319 & 7 & 6243 & 11 & 0.96667 & 0.00112 & 0.99888 & 0.03333 \\ 2 & 328 & 5 & 6245 & 2 & 0.99394 & 0.00080 & 0.99920 & 0.00606 \\ 3 & 329 & 4 & 6246 & 1 & 0.99697 & 0.00064 & 0.99936 & 0.00303 \\ 4 & 330 & 8 & 6242 & 0 & 1.00000 & 0.00128 & 0.99872 & 0.00000 \\ 5 & 316 & 5 & 6245 & 14 & 0.95758 & 0.00080 & 0.99920 & 0.04242 \\ 6 & 324 & 5 & 6245 & 6 & 0.98182 & 0.00080 & 0.99920 & 0.01818 \\ 7 & 328 & 5 & 6245 & 2 & 0.99394 & 0.00080 & 0.99920 & 0.00606 \\ 8 & 325 & 4 & 6246 & 5 & 0.98485 & 0.00064 & 0.99936 & 0.01515 \\ 9 & 325 & 8 & 6242 & 5 & 0.98485 & 0.00128 & 0.99872 & 0.01515 \\ 10 & 293 & 4 & 6246 & 37 & 0.88788 & 0.00064 & 0.99936 & 0.11212 \\ 11 & 323 & 3 & 6247 & 7 & 0.97879 & 0.00048 & 0.99952 & 0.02121 \\ 12 & 319 & 4 & 6246 & 11 & 0.96667 & 0.00064 & 0.99936 & 0.03333 \\ 13 & 328 & 6 & 6244 & 2 & 0.99394 & 0.00096 & 0.99904 & 0.00606 \\ 14 & 318 & 4 & 6246 & 12 & 0.96364 & 0.00064 & 0.99936 & 0.03636 \\ 15 & 321 & 4 & 6246 & 9 & 0.97273 & 0.00064 & 0.99936 & 0.02727 \\ 16 & 324 & 5 & 6245 & 6 & 0.98182 & 0.00080 & 0.99920 & 0.01818\end{array}$




$\begin{array}{rrrrrrrrr}17 & 325 & 4 & 6246 & 5 & 0.98485 & 0.00064 & 0.99936 & 0.01515 \\ 18 & 320 & 4 & 6246 & 10 & 0.96970 & 0.00064 & 0.99936 & 0.03030 \\ 19 & 314 & 5 & 6245 & 16 & 0.95152 & 0.00080 & 0.99920 & 0.04848 \\ 20 & 308 & 5 & 6245 & 22 & 0.93333 & 0.00080 & 0.99920 & 0.06667\end{array}$

Experiment Number (\#)

True Positive (TP)

False Positive (FP)

True Negative (TN)

False Negative (FN)

True Positive Rate (TPR)

False Positive Rate (FPR)

True Negative Rate (TNR)

False Negative Rate (FNR)

Table 8b. Differential Gene Expression (DGE) algorithm differential maximum inclusion method accuracy performance analysis using the confusion matrix classification system for the downregulated genes.

\begin{tabular}{|c|c|c|c|c|c|c|}
\hline$\#$ & PPV & NPV & $\mathrm{AC}$ & GM1 & GM2 & F1M \\
\hline 1 & 0.97853 & 0.99824 & 0.99726 & 0.97258 & 0.98264 & 0.97256 \\
\hline 2 & 0.98498 & 0.99968 & 0.99894 & 0.98945 & 0.99657 & 0.98944 \\
\hline 3 & 0.98799 & 0.99984 & 0.99924 & 0.99247 & 0.99816 & 0.99246 \\
\hline 4 & 0.97633 & 1.00000 & 0.99878 & 0.98809 & 0.99936 & 0.98802 \\
\hline 5 & 0.98442 & 0.99776 & 0.99711 & 0.97091 & 0.97817 & 0.97081 \\
\hline 6 & 0.98480 & 0.99904 & 0.99833 & 0.98331 & 0.99047 & 0.98331 \\
\hline 7 & 0.98498 & 0.99968 & 0.99894 & 0.98945 & 0.99657 & 0.98944 \\
\hline 8 & 0.98784 & 0.99920 & 0.99863 & 0.98634 & 0.99208 & 0.98634 \\
\hline 9 & 0.97598 & 0.99920 & 0.99802 & 0.98040 & 0.99176 & 0.98039 \\
\hline 10 & 0.98653 & 0.99411 & 0.99377 & 0.93591 & 0.94197 & 0.93461 \\
\hline 11 & 0.99080 & 0.99888 & 0.99848 & 0.98477 & 0.98910 & 0.98476 \\
\hline 12 & 0.98762 & 0.99824 & 0.99772 & 0.97709 & 0.98288 & 0.97703 \\
\hline 13 & 0.98204 & 0.99968 & 0.99878 & 0.98797 & 0.99649 & 0.98795 \\
\hline 14 & 0.98758 & 0.99808 & 0.99757 & 0.97553 & 0.98134 & 0.97546 \\
\hline 15 & 0.98769 & 0.99856 & 0.99802 & 0.98018 & 0.98595 & 0.98015 \\
\hline 16 & 0.98480 & 0.99904 & 0.99833 & 0.98331 & 0.99047 & 0.98331 \\
\hline 17 & 0.98784 & 0.99920 & 0.99863 & 0.98634 & 0.99208 & 0.98634 \\
\hline 18 & 0.98765 & 0.99840 & 0.99787 & 0.97863 & 0.98442 & 0.97859 \\
\hline 19 & 0.98433 & 0.99744 & 0.99681 & 0.96778 & 0.97507 & 0.96764 \\
\hline 20 & 0.98403 & 0.99649 & 0.99590 & 0.95834 & 0.96571 & 0.95801 \\
\hline
\end{tabular}


Experiment Number (\#)

Positive Predictive Value (PPV)

Negative Predictive Value (NPV)

Accuracy (AC)

Geometric-Mean $_{1}$ (GM1)

Geometric-Mean 2 (GM2)

$\mathrm{F}_{1}$-Measure (F1M)

Table 9a. Differential Gene Expression (DGE) algorithm differential maximum inclusion method accuracy performance analysis using the confusion matrix classification system for the upregulated genes and the downregulated genes.

$\begin{array}{rrrrrllll}\text { \# } & \text { TP } & \text { FP } & \text { TN } & \text { FN } \text { TPR } & \text { FPR } & \text { TNR } & \text { FNR } \\ & & & & & & & & \\ 1 & 645 & 13 & 5907 & 15 & 0.97727 & 0.00220 & 0.99780 & 0.02273 \\ 2 & 641 & 12 & 5908 & 19 & 0.97121 & 0.00203 & 0.99797 & 0.02879 \\ 3 & 648 & 11 & 5909 & 12 & 0.98182 & 0.00186 & 0.99814 & 0.01818 \\ 4 & 658 & 14 & 5906 & 2 & 0.99697 & 0.00236 & 0.99764 & 0.00303 \\ 5 & 624 & 10 & 5910 & 36 & 0.94545 & 0.00169 & 0.99831 & 0.05455 \\ 6 & 650 & 11 & 5909 & 10 & 0.98485 & 0.00186 & 0.99814 & 0.01515 \\ 7 & 644 & 12 & 5908 & 16 & 0.97576 & 0.00203 & 0.99797 & 0.02424 \\ 8 & 654 & 11 & 5909 & 6 & 0.99091 & 0.00186 & 0.99814 & 0.00909 \\ 9 & 649 & 14 & 5906 & 11 & 0.98333 & 0.00236 & 0.99764 & 0.01667 \\ 10 & 621 & 11 & 5909 & 39 & 0.94091 & 0.00186 & 0.99814 & 0.05909 \\ 11 & 650 & 10 & 5910 & 10 & 0.98485 & 0.00169 & 0.99831 & 0.01515 \\ 12 & 634 & 10 & 5910 & 26 & 0.96061 & 0.00169 & 0.99831 & 0.03939 \\ 13 & 649 & 12 & 5908 & 11 & 0.98333 & 0.00203 & 0.99797 & 0.01667 \\ 14 & 643 & 10 & 5910 & 17 & 0.97424 & 0.00169 & 0.99831 & 0.02576 \\ 15 & 647 & 12 & 5908 & 13 & 0.98030 & 0.00203 & 0.99797 & 0.01970 \\ 16 & 653 & 13 & 5907 & 7 & 0.98939 & 0.00220 & 0.99780 & 0.01061 \\ 17 & 639 & 10 & 5910 & 21 & 0.96818 & 0.00169 & 0.99831 & 0.03182 \\ 18 & 633 & 10 & 5910 & 27 & 0.95909 & 0.00169 & 0.99831 & 0.04091 \\ 19 & 635 & 9 & 5911 & 25 & 0.96212 & 0.00152 & 0.99848 & 0.03788 \\ 20 & 623 & 12 & 5908 & 37 & 0.94394 & 0.00203 & 0.99797 & 0.05606\end{array}$

Experiment Number (\#)

True Positive (TP)

False Positive (FP)

True Negative (TN)

False Negative (FN)

True Positive Rate (TPR)

False Positive Rate (FPR)

True Negative Rate (TNR) 
False Negative Rate (FNR)

Table 9b. Differential Gene Expression (DGE) algorithm differential maximum inclusion method accuracy performance analysis using the confusion matrix classification system for the upregulated genes and the downregulated genes.

$\begin{array}{llllllll}\text { \# } & \text { PPV } & \text { NPV } & \text { AC } & \text { GM1 } & \text { GM2 } & \text { F1M } \\ & & & & & & & \\ 1 & & & & & & \\ 1 & 0.98024 & 0.99747 & 0.99574 & 0.97876 & 0.98749 & 0.97876 \\ 2 & 0.98162 & 0.99679 & 0.99529 & 0.97640 & 0.98450 & 0.97639 \\ 3 & 0.98331 & 0.99797 & 0.99650 & 0.98256 & 0.98995 & 0.98256 \\ 4 & 0.97917 & 0.99966 & 0.99757 & 0.98803 & 0.99730 & 0.98799 \\ 5 & 0.98423 & 0.99395 & 0.99301 & 0.96465 & 0.97152 & 0.96445 \\ 6 & 0.98336 & 0.99831 & 0.99681 & 0.98410 & 0.99147 & 0.98410 \\ 7 & 0.98171 & 0.99730 & 0.99574 & 0.97873 & 0.98680 & 0.97872 \\ 8 & 0.98346 & 0.99899 & 0.99742 & 0.98718 & 0.99452 & 0.98717 \\ 9 & 0.97888 & 0.99814 & 0.99620 & 0.98111 & 0.99046 & 0.98110 \\ 10 & 0.98259 & 0.99344 & 0.99240 & 0.96153 & 0.96910 & 0.96130 \\ 11 & 0.98485 & 0.99831 & 0.99696 & 0.98485 & 0.99156 & 0.98485 \\ 12 & 0.98447 & 0.99562 & 0.99453 & 0.97247 & 0.97928 & 0.97239 \\ 13 & 0.98185 & 0.99814 & 0.99650 & 0.98259 & 0.99063 & 0.98259 \\ 14 & 0.98469 & 0.99713 & 0.99590 & 0.97945 & 0.98620 & 0.97944 \\ 15 & 0.98179 & 0.99780 & 0.99620 & 0.98105 & 0.98910 & 0.98105 \\ 16 & 0.98048 & 0.99882 & 0.99696 & 0.98493 & 0.99359 & 0.98492 \\ 17 & 0.98459 & 0.99646 & 0.99529 & 0.97635 & 0.98313 & 0.97632 \\ 18 & 0.98445 & 0.99545 & 0.99438 & 0.97169 & 0.97850 & 0.97160 \\ 19 & 0.98602 & 0.99579 & 0.99483 & 0.97400 & 0.98013 & 0.97392 \\ 20 & 0.98110 & 0.99378 & 0.99255 & 0.96234 & 0.97058 & 0.96216\end{array}$

Experiment Number (\#)

Positive Predictive Value (PPV)

Negative Predictive Value (NPV)

Accuracy (AC)

Geometric-Mean $_{1}(\mathrm{GM} 1)$

Geometric-Mean 2 (GM2)

$\mathrm{F}_{1}$-Measure $(\mathrm{F} 1 \mathrm{M})$

Refer to the DGE algorithm differential maximum inclusion method accuracy performance analysis using the confusion matrix classification system for the upregulated genes (Tables 7a and 7b). For the 330 known upregulated differentially-expressed genes, the DGE algorithm generated fourteen performance statistics: 1 . TP 308 to 329,2 . FP 4 to 
8, 3. TN 6242 to 6246,4 . FN 1 to 22,5 . TPR 0.93333 to 0.99697 normalized fraction, 6 . FPR 0.00064 to 0.00128 normalized fraction, 7. TNR 0.99872 to 0.99936 normalized fraction, 8. FNR 0.00303 to 0.06667 normalized fraction, 9. PPV 0.97605 to 0.98769 normalized fraction, 10. NPV 0.99649 to 0.99984 normalized fraction, 11. AC 0.99590 to 0.99878 normalized fraction, 12 . GM1 0.95834 to 0.99803 normalized fraction, 13. GM2 0.96571 to 0.99792 normalized fraction and 14. F1M 0.95801 to 0.98799 normalized fraction.

Refer to the DGE algorithm differential maximum inclusion method accuracy performance analysis using the confusion matrix classification system for the downregulated genes (Tables 8a and 8b). For the 330 known downregulated differentially-expressed genes, the DGE algorithm generated fourteen performance statistics: 1. TP 293 to 330,2 . FP 3 to 8, 3. TN 6242 to 6247,4 . FN 0 to 37, 5. TPR 0.88788 to 1.00000 normalized fraction, 6 . FPR 0.00048 to 0.00128 normalized fraction, 7. TNR 0.99872 to 0.99952 normalized fraction, 8. FNR 0.00000 to 0.11212 normalized fraction, 9. PPV 0.97598 to 0.99080 normalized fraction, 10. NPV 0.99411 to 1.00000 normalized fraction, 11. AC 0.99377 to 0.99924 normalized fraction, 12. GM1 0.93591 to 0.99247 normalized fraction, 13. GM2 0.94197 to 0.99936 normalized fraction and 14 . F1M 0.93461 to 0.99246 normalized fraction.

Refer to the DGE algorithm differential maximum inclusion method accuracy performance analysis using the confusion matrix classification system for the upregulated genes and the downregulated genes (Tables $9 a$ and $9 b$ ). For the 330 known upregulated differentially-expressed genes and the 330 known downregulated differentially-expressed genes combined, the DGE algorithm generated fourteen performance statistics: 1. TP 621 to 658,2 . FP 9 to 14,3 . TN 5906 to 5911, 4. FN 2 to 39, 5. TPR 0.94091 to 0.99697 normalized fraction, 6. FPR 0.00152 to 0.00236 normalized fraction, 7. TNR 0.99764 to 0.99848 normalized fraction, 8. FNR 0.00303 to 0.05909 normalized fraction, 9. PPV 0.97888 to 0.98485 normalized fraction, 10. NPV 0.99344 to 0.99966 normalized fraction, 11. AC 0.99240 to 0.99757 normalized fraction, 12. GM1 0.96153 to 0.98803 
normalized fraction, 13. GM2 0.96910 to 0.99730 normalized fraction and 14. F1M 0.96130 to 0.98799 normalized fraction.

The differential maximum inclusion method accuracy performance analysis based on the confusion matrix classification system confirmed that using the differential maximum inclusion method in the DGE algorithm was the most accurate method.

\subsection{Comparative Precision Performance Analysis}

\subsubsection{Statistical Procedure}

The Significance Analysis of Microarrays (SAM) algorithm and the Differential Gene Expression (DGE) algorithm were subjected to comparative precision performance analysis. The comparative precision performance analysis was based on the analysis of microarray datasets containing differentially-expressed genes. The microarray datasets underwent $\log$ transformation, Cartesian coordinate calculation and normalization. The comparative precision performance analysis consisted of seven procedures:

1. The Gene Expression Omnibus (GEO) repository at the National Center for Biotechnology Information (NCBI) archived and distributed high-throughput molecular abundance data. The high-throughput molecular abundance data consisted primarily of gene expression data generated by microarray technologies. The GEO database has emerged as the leading compendium for the dissemination of gene expression data (Edgar et al. 2002; Barrett et al. 2005; Barrett and Edgar 2006a, 2006b; Barrett et al. 2007). The GSE3158 series of murine retinoblastoma tumor microarray datasets was retrieved from the GEO database (Black et al. 2003; Huang et al. 2003). The GSE3158 series of murine retinoblastoma tumor microarray datasets included four murine wild-type tissue microarray datasets: 1. GSM70688, 2. GSM70689, 3. GSM70690 and 4. GSM70691. The four murine wild-type tissue microarray datasets corresponded to four replicated microarray datasets. The GSE3158 series of murine retinoblastoma tumor microarray datasets included four murine retinoblastoma knockout tumor tissue microarray datasets: 
1. GSM70703, 2. GSM70704, 3. GSM70705 and 4. GSM70706. The four murine retinoblastoma knockout tumor tissue microarray datasets corresponded to four replicated microarray datasets. The two-condition experiment corresponded to eight replicated microarray datasets.

2. The two-condition experiment corresponding to eight replicated microarray datasets was divided into two states: 1 . control state and 2. experimental state. The control state consisted of four replicated microarray datasets: 1. GSM70688, 2. GSM70689, 3. GSM70690 and 4. GSM70691. The experimental state consisted of four replicated microarray datasets: 1. GSM70703, 2. GSM70704, 3. GSM70705 and 4. GSM70706. The two-condition experiment was composed of two states in which each state corresponded to four replicated microarray datasets. The two-condition experiment contained differentially-expressed genes.

3. One subset of three replicated microarray datasets was chosen from the set of four replicated microarray datasets which formed the control state. The subset of three replicated microarray datasets formed the control state for one comparative precision performance analysis experiment. One subset of three replicated microarray datasets was chosen from the set of four replicated microarray datasets which formed the experimental state. The subset of three replicated microarray datasets formed the experimental state for one comparative precision performance analysis experiment.

4. The SAM algorithm and the DGE algorithm were applied to the microarray datasets which contained differentially-expressed genes. The SAM algorithm and the DGE algorithm assigned differential ranks to the genes.

5. The comparative precision performance analysis consisted of $\mathrm{C}(4,3) * \mathrm{C}(4,3)=16$ repeated experiments.

6. The variances of the differential ranks generated by the sixteen SAM algorithm computations were computed. The variances of the differential ranks generated by the 
sixteen DGE algorithm computations were computed. The $\log _{\mathrm{e}}$ (variance DGE $_{\text {I }} /$ variance $_{\mathrm{SAM}}$ ) ratios were computed.

7. The distribution of the $\log _{\mathrm{e}}$ ( variance $_{\mathrm{DGE}} /$ variance $_{\mathrm{SAM}}$ ) ratios was computed using eight statistical procedures: 1 . minimum, 2. first quartile, 3. mean, 4. median, 5. third quartile, 6. maximum, 7. frequency distribution and 8. histogram.

\subsubsection{Microarray Datasets}

The microarray datasets each contained differentially-expressed genes and nondifferentially-expressed genes which formed a total of 6560 genes. 


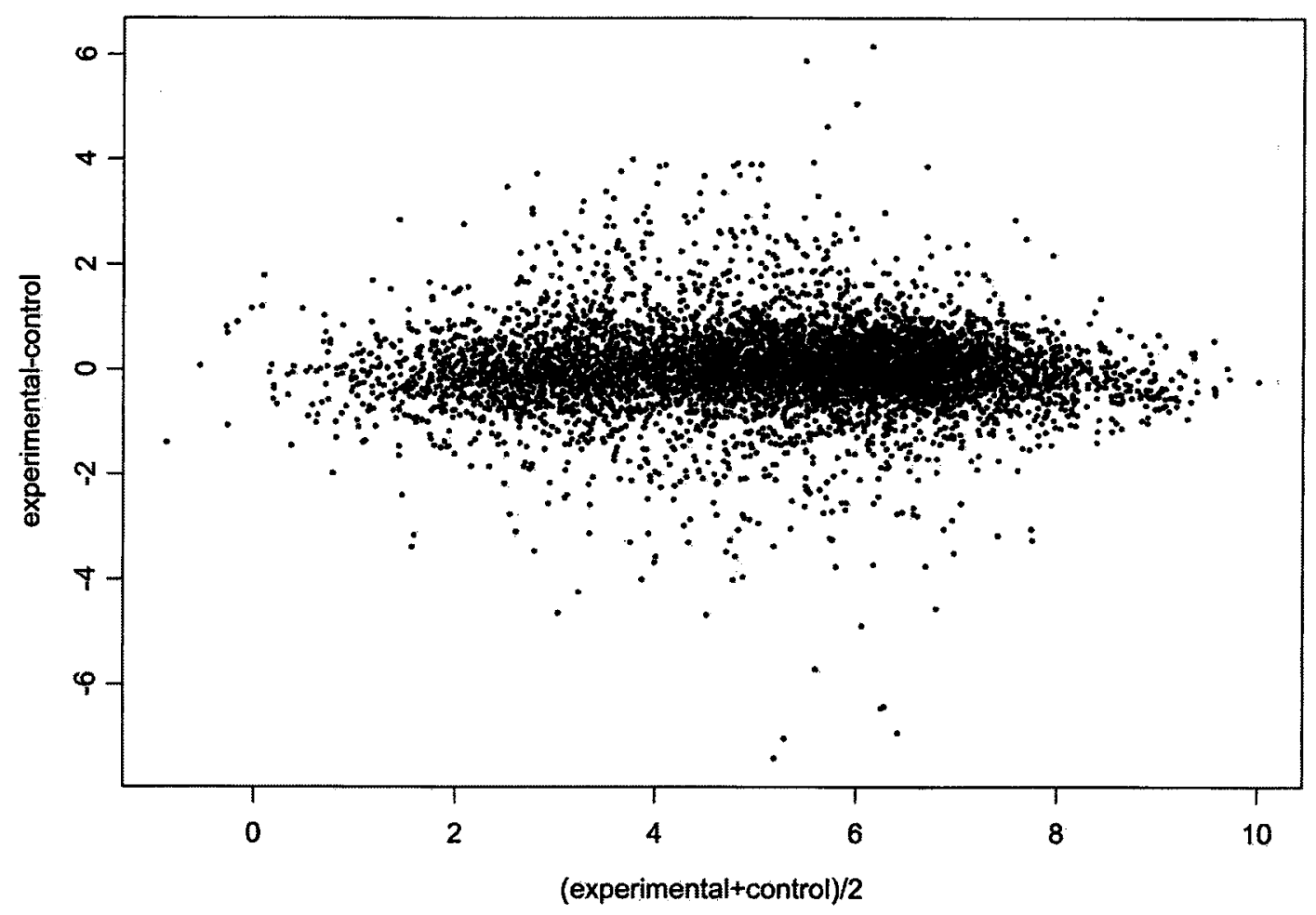

Figure 21. Differential gene expression graph. The datapoints consisted of actual data rather than synthetic data. 


\subsubsection{Significance Analysis of Microarrays (SAM) Algorithm}

The precision performance analysis was used for the investigation of the Significance Analysis of Microarrays (SAM) algorithm precision. Refer to the SAM algorithm difference statistic graph and expected difference statistic graph (Figure 23). The SAM algorithm exhibited a nonsymmetric crossover point. The SAM algorithm demonstrated a nonsymmetric differential distribution between the lower difference statistics and the higher difference statistics. The lower difference statistics exhibited larger differentials and the higher difference statistics exhibited smaller differentials. 
SAM

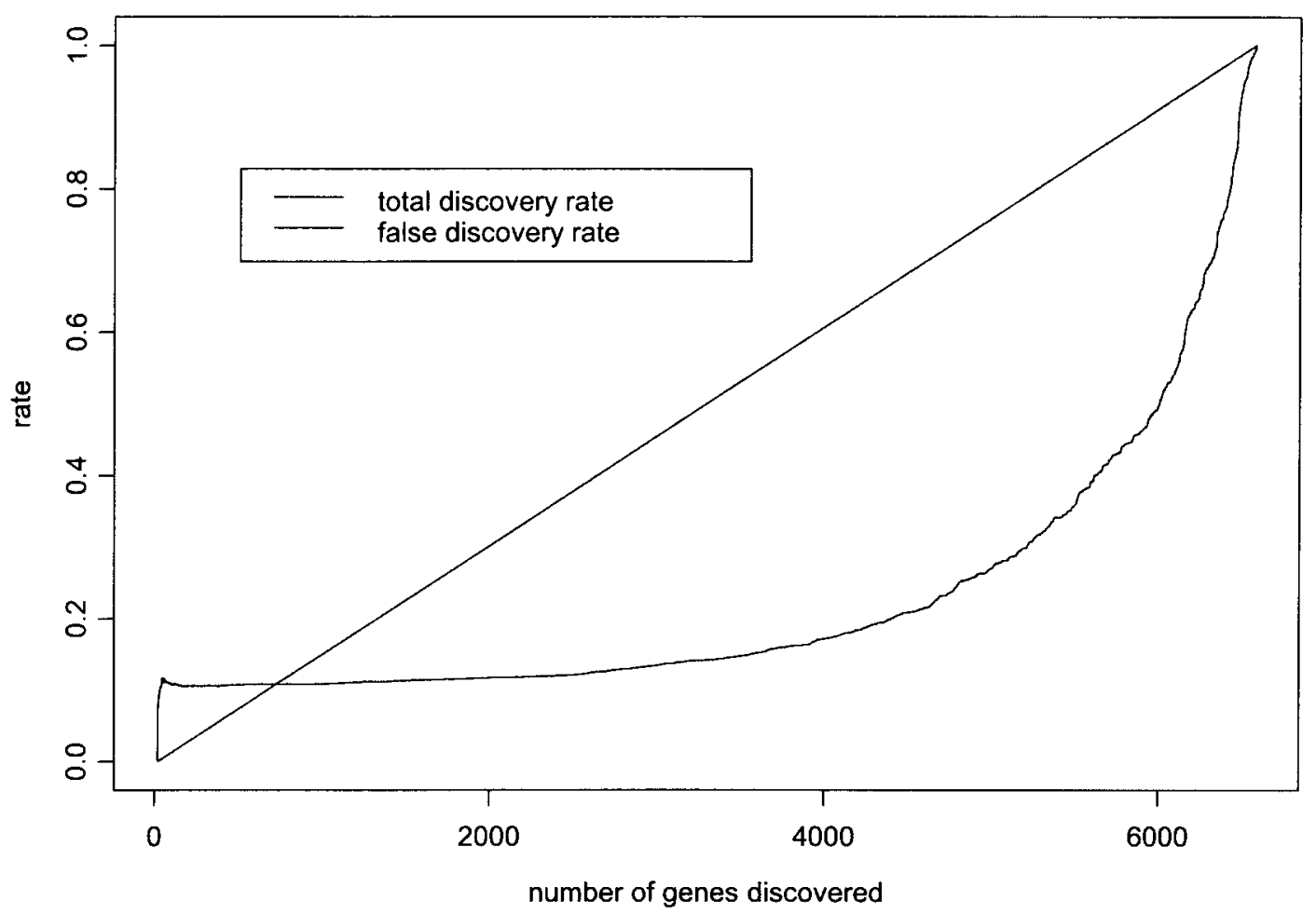

Figure 22. Significance Analysis of Microarrays (SAM) algorithm total discovery rate (TDR) graph and false discovery rate (FDR) graph. The TDR graph was represented as the green line. The FDR graph was represented as the red line. 


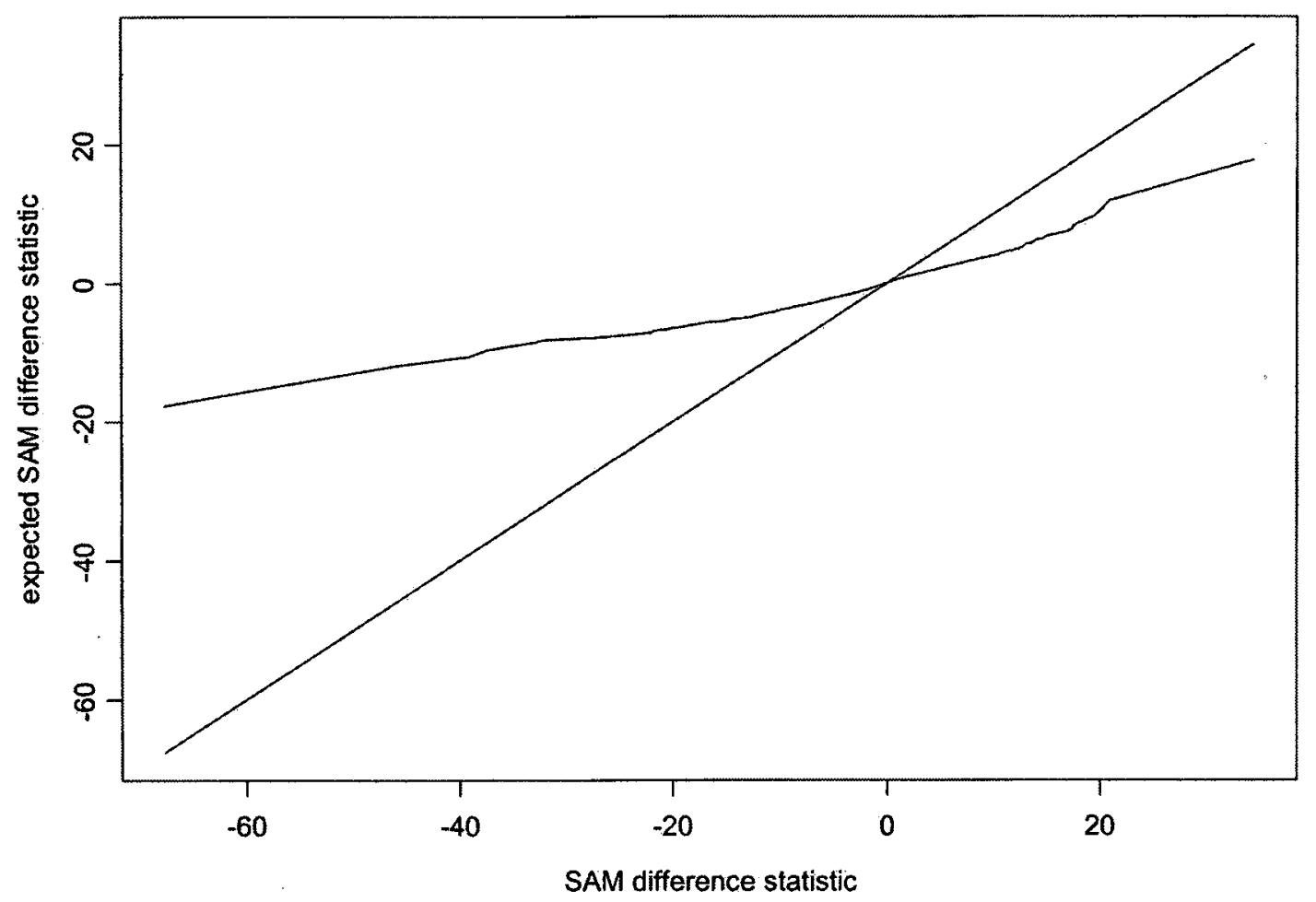

Figure 23. Significance Analysis of Microarrays (SAM) algorithm difference statistic graph and expected difference statistic graph. The nonsymmetric crossover point was represented as the noncentral intersection point between the two intersecting graphs. The nonsymmetric differentials were represented as the nonequal deviations at the extrema between the two intersecting graphs. 


\subsubsection{Differential Gene Expression (DGE) Algorithm}

The precision performance analysis was used for the investigation of the Differential Gene Expression (DGE) algorithm precision. Refer to the DGE algorithm quantile graph and expected quantile graph (Figure 25). Refer to the DGE algorithm quantile graph and revised expected quantile graph (Figure 26). The DGE algorithm exhibited an approximately symmetric crossover point. The DGE algorithm demonstrated an approximately symmetric differential distribution between the lower quantiles and the higher quantiles. The lower quantiles and the higher quantiles exhibited approximately equal differentials. 


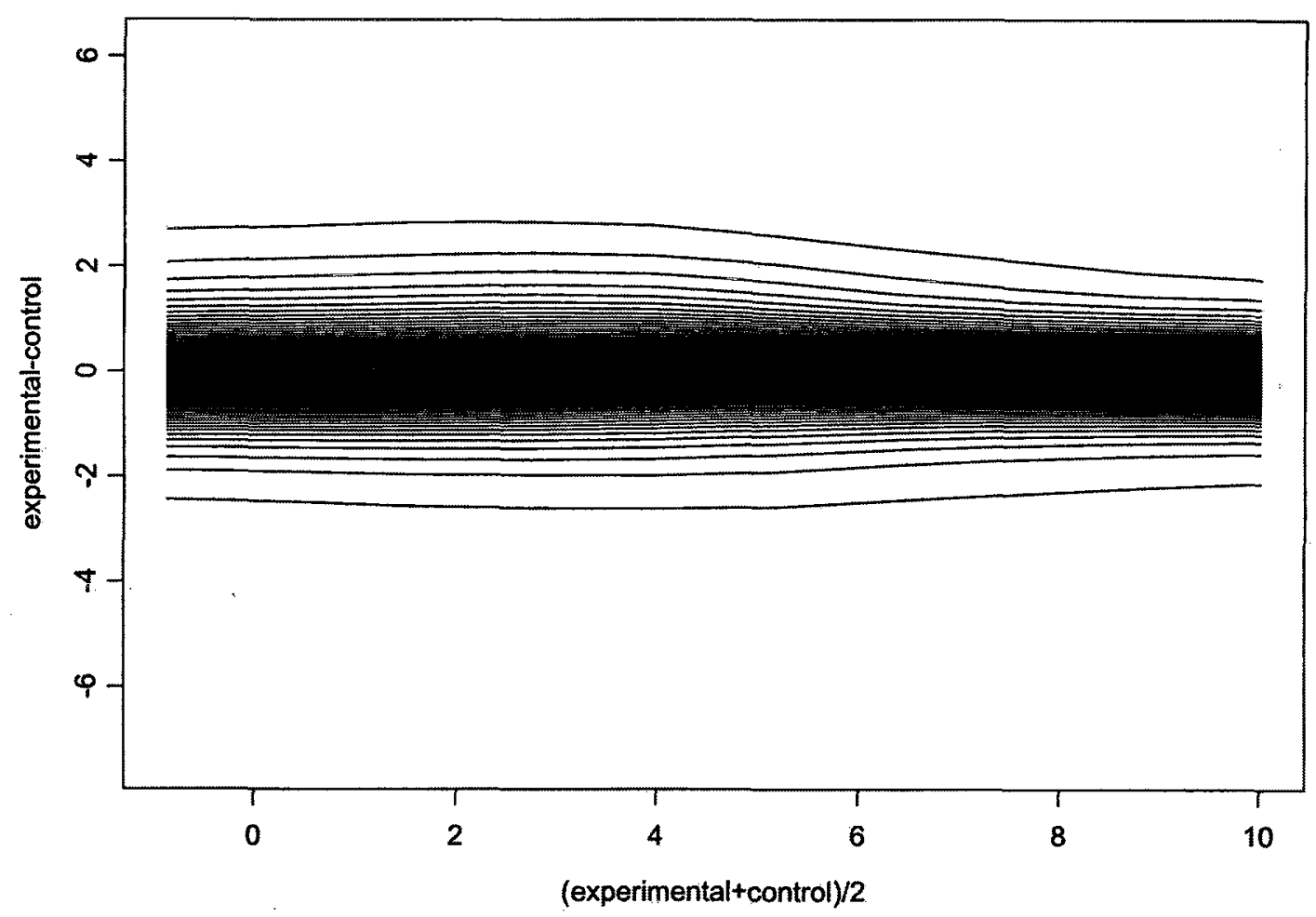

Figure 24. Differential Gene Expression (DGE) algorithm nonlinear regression quantile graphs. The ninety nine nonlinear regression quantile graphs were calculated using the $\mathrm{Yu}$ and Jones (1998) algorithm. The ninety nine nonlinear regression quantile graphs provided the basis for the identification of the upregulated genes and the downregulated genes. 


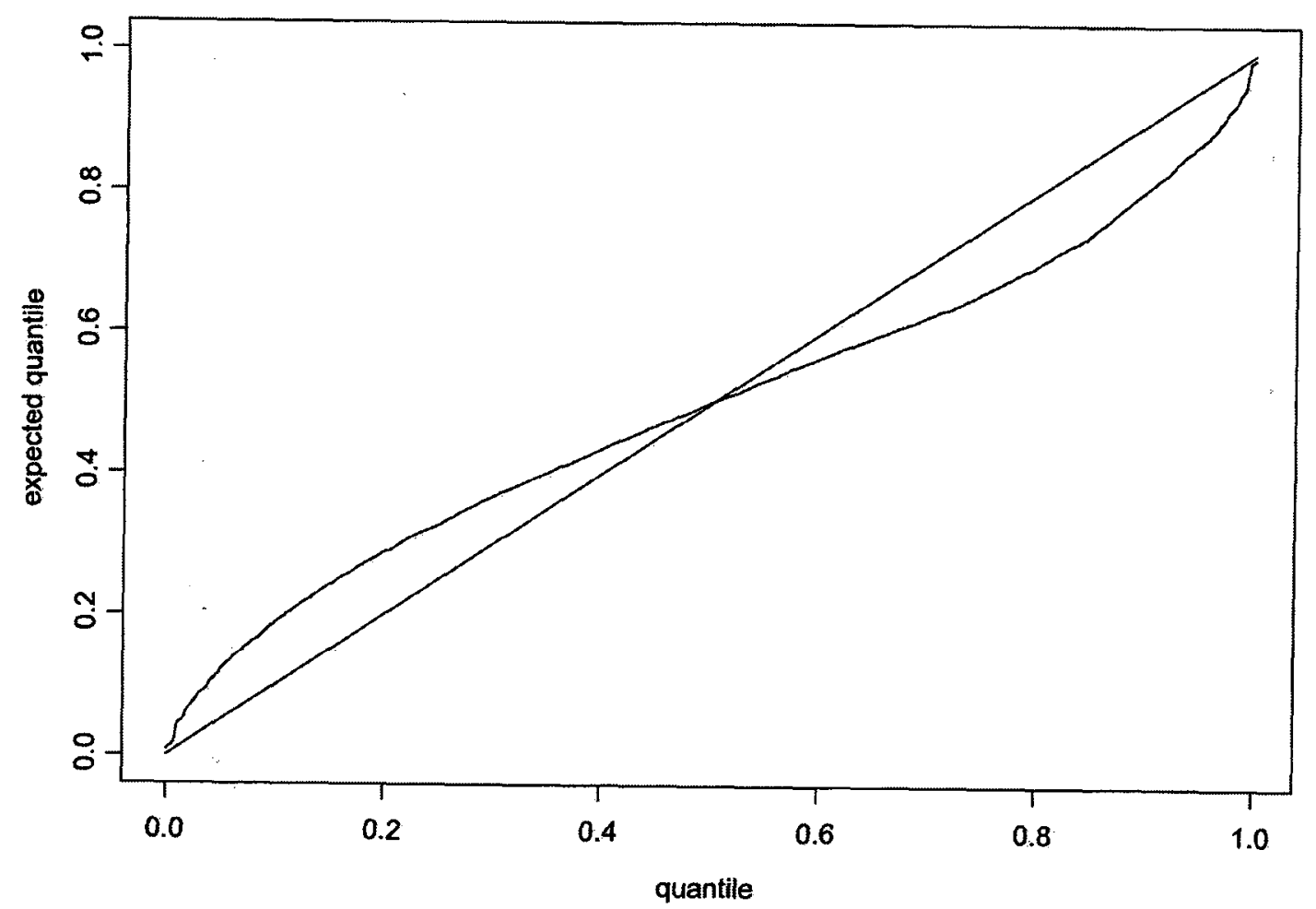

Figure 25. Differential Gene Expression (DGE) algorithm quantile graph and expected quantile graph. The quantile graph was represented as the straight line. The expected quantile graph was represented as the curved line. 


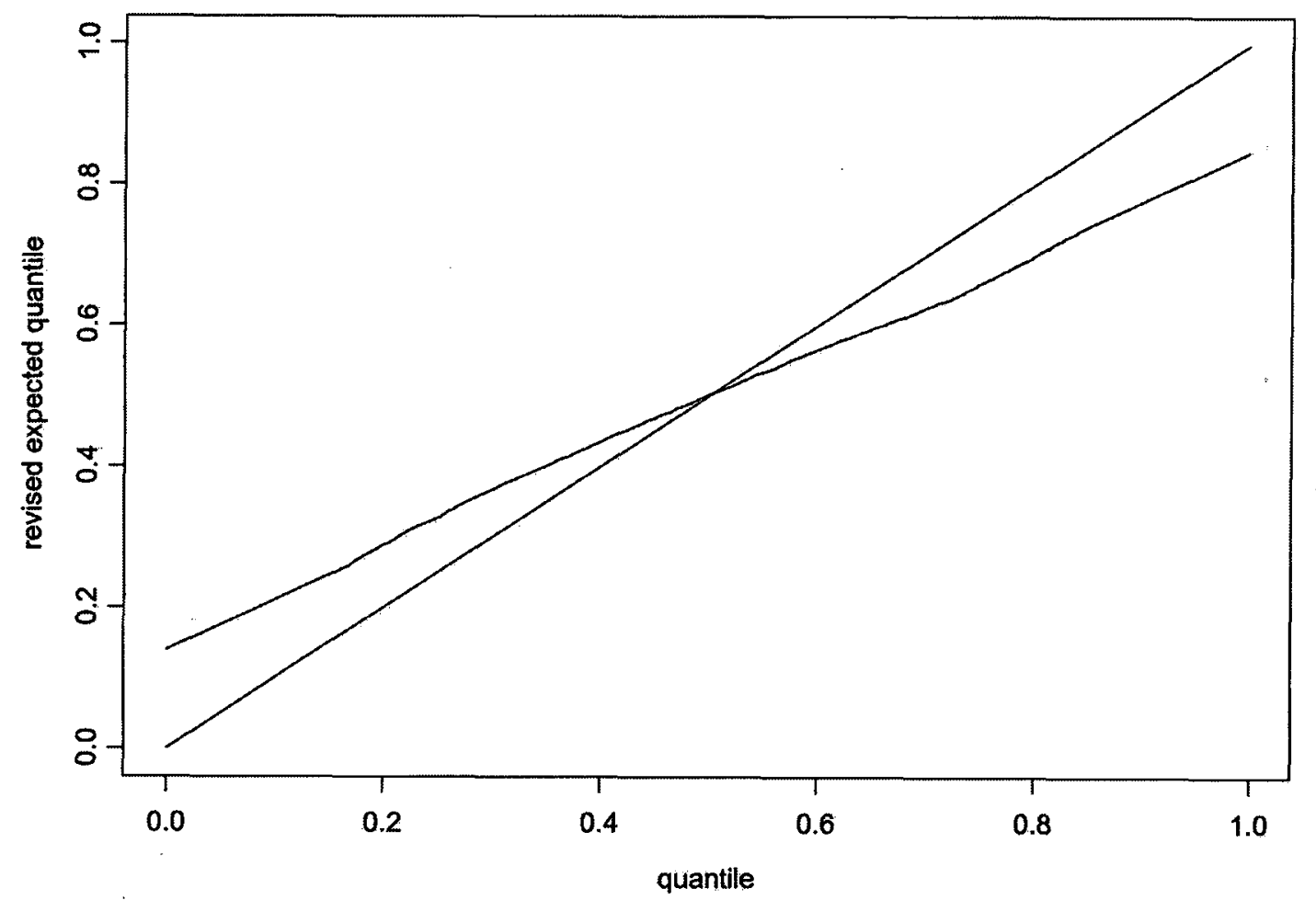

Figure 26. Differential Gene Expression (DGE) algorithm quantile graph and revised expected quantile graph. The quantile graph was represented as the straight line. The revised expected quantile graph was represented as the curved line. 


\subsubsection{Statistical Results}

Table 10. $\log _{\mathrm{e}}\left(\right.$ variance $_{\mathrm{DGE}} /$ variance $_{\mathrm{SAM}}$ ) ratio statistics.

$\begin{array}{cccccc}\text { MIN } & \text { Q1 } & \text { MN } & \text { MD } & \text { Q3 } & \text { MAX } \\ -10.88853 & -1.08847 & -0.49964 & -0.41199 & 0.27802 & 8.47586\end{array}$

Minimum (MIN)

First Quartile (Q1)

Mean (MN)

Median (MD)

Third Quartile (Q3)

Maximum (MAX)

Refer to the $\log _{\mathrm{e}}$ ( variance $_{\mathrm{DGE}} /$ variance $_{\mathrm{SAM}}$ ) ratio statistics (Table 10). The distribution of the $\log _{\mathrm{e}}$ (variance DGE $_{\text {/ variance }}$ SAM) ratios was summarized using the mean

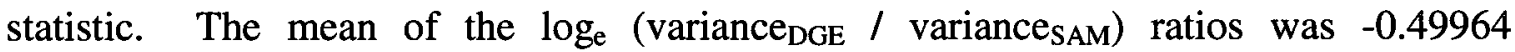
dimensionless units. The antilogarithm of the mean of the $\log _{e}$ (variance DGE $/$ variance $_{\mathrm{SAM}}$ ) ratios was $\mathrm{e}^{-0.49964}=0.60675$ normalized fraction. The mean size of the variance $_{\mathrm{DGE}}$ was 0.60675 normalized fraction of the mean size of the variance SAM. The $_{\text {S }}$ mean size of the variance DGE $_{\text {was }} 1.00000-0.60675=0.39325$ normalized fraction smaller than the mean size of the variance ${ }_{\mathrm{SAM}}$.

Refer to the $\log _{\mathrm{e}}$ ( variance $_{\mathrm{DGE}} /$ variance $_{\mathrm{SAM}}$ ) ratio statistics (Table 10). The distribution of the $\log _{\mathrm{e}}$ ( variance $_{\mathrm{DGE}} /$ variance $_{\mathrm{SAM}}$ ) ratios was summarized using the median statistic. The median of the $\log _{e}$ (variance DGE $_{\text {/ variance }}$ AM) ratios was -0.41199 dimensionless units. The antilogarithm of the median of the $\log _{\mathrm{e}}$ (variance DGE $_{\text {I }} /$ variance $_{\mathrm{SAM}}$ ) ratios was $\mathrm{e}^{-0.41199}=0.66233$ normalized fraction. The median size of the variance $_{\text {DGE }}$ was 0.66233 normalized fraction of the median size of the variance SAM. The $_{\text {. The }}$ median size of the variance DGE $_{\text {was }} 1.00000-0.66233=0.33767$ normalized fraction smaller than the median size of the variance SAM. $_{\text {. }}$ 
Table 11. $\log _{\mathrm{e}}$ (variance $\mathrm{DGE} /$ variance $_{\mathrm{SAM}}$ ) ratio frequency distribution.

\begin{tabular}{|c|c|c|c|c|c|c|c|c|c|}
\hline C & LB & & & UB & & & LLB & LUB & $\mathrm{CF}$ \\
\hline 1 & 1.670170 & $\mathrm{x}$ & $10^{-5}$ & 2.753645 & $\mathrm{x}$ & $10^{-5}$ & -11.0 & -10.5 & 1 \\
\hline 2 & 2.753645 & $\mathrm{x}$ & $10^{-5}$ & 4.539993 & $\mathrm{x}$ & $10^{-5}$ & -10.5 & -10.0 & 0 \\
\hline 3 & 4.539993 & $\mathbf{x}$ & $10^{-5}$ & 7.485183 & $\mathbf{x}$ & $10^{-5}$ & -10.0 & -9.5 & 0 \\
\hline 4 & 7.485183 & $\mathrm{x}$ & $10^{-5}$ & 1.234098 & $\mathrm{x}$ & $10^{-4}$ & -9.5 & -9.0 & 0 \\
\hline 5 & 1.234098 & $\mathrm{x}$ & $10^{-4}$ & 2.034684 & $\mathrm{x}$ & $10^{-4}$ & -9.0 & -8.5 & 0 \\
\hline 6 & 2.034684 & $\mathbf{x}$ & $10^{-4}$ & 3.354626 & $\mathbf{x}$ & $10^{-4}$ & -8.5 & -8.0 & 5 \\
\hline 7 & 3.354626 & $x$ & $10^{-4}$ & 5.530844 & $x$ & $10^{-4}$ & -8.0 & -7.5 & 4 \\
\hline 8 & 5.530844 & $\mathrm{x}$ & $10^{-4}$ & 9.118820 & $\mathrm{x}$ & $10^{-4}$ & -7.5 & -7.0 & 5 \\
\hline 9 & 9.118820 & $\mathrm{x}$ & $10^{-4}$ & 1.503439 & $\mathrm{x}$ & $10^{-3}$ & -7.0 & -6.5 & 6 \\
\hline 10 & 1.503439 & $x$ & $10^{-3}$ & 2.478752 & $x$ & $10^{-3}$ & -6.5 & -6.0 & 11 \\
\hline 11 & 2.478752 & $\mathrm{x}$ & $10^{-3}$ & 4.086771 & $\mathrm{x}$ & $10^{-3}$ & -6.0 & -5.5 & 21 \\
\hline 12 & 4.086771 & $\mathrm{x}$ & $10^{-3}$ & 6.737947 & $\mathrm{x}$ & $10^{-3}$ & -5.5 & -5.0 & 14 \\
\hline 13 & 6.737947 & $\mathbf{x}$ & $10^{-3}$ & 1.110900 & $\mathbf{x}$ & $10^{-2}$ & -5.0 & -4.5 & 23 \\
\hline 14 & 1.110900 & $x$ & $10^{-2}$ & 1.831564 & $x$ & $10^{-2}$ & -4.5 & -4.0 & 45 \\
\hline 15 & 1.831564 & $\mathrm{x}$ & $10^{-2}$ & 3.019738 & $x$ & $10^{-2}$ & -4.0 & -3.5 & 49 \\
\hline 16 & 3.019738 & $x$ & $10^{-2}$ & 4.978707 & $\mathrm{x}$ & $10^{-2}$ & -3.5 & -3.0 & 53 \\
\hline 17 & 4.978707 & $\mathrm{x}$ & $10^{-2}$ & 8.208500 & $\mathrm{x}$ & $10^{-2}$ & -3.0 & -2.5 & 116 \\
\hline 18 & 8.208500 & $\mathrm{x}$ & $10^{-2}$ & 1.353353 & $\mathrm{x}$ & $10^{-1}$ & -2.5 & -2.0 & 193 \\
\hline 19 & 1.353353 & $x$ & $10^{-1}$ & 2.231302 & $x$ & $10^{-1}$ & -2.0 & -1.5 & 421 \\
\hline 20 & 2.231302 & $\mathrm{x}$ & $10^{-1}$ & 3.678794 & $\mathrm{x}$ & $10^{-1}$ & -1.5 & -1.0 & 841 \\
\hline 21 & 3.678794 & $x$ & $10^{-1}$ & 6.065307 & $x$ & $10^{-1}$ & -1.0 & -0.5 & 1225 \\
\hline 22 & 6.065307 & $\mathrm{x}$ & $10^{-1}$ & 1.000000 & $\mathrm{x}$ & $10^{+0}$ & -0.5 & 0.0 & 1289 \\
\hline 23 & 1.000000 & $\mathrm{x}$ & $10^{+0}$ & 1.648721 & $\mathrm{x}$ & $10^{+0}$ & 0.0 & 0.5 & 1005 \\
\hline 24 & 1.648721 & $\mathrm{x}$ & $10^{+0}$ & 2.718282 & $\mathrm{x}$ & $10^{+0}$ & 0.5 & 1.0 & 709 \\
\hline 25 & 2.718282 & $\mathrm{x}$ & $10^{+0}$ & 4.481689 & $\mathrm{x}$ & $10^{+0}$ & 1.0 & 1.5 & 348 \\
\hline 26 & 4.481689 & $\mathrm{x}$ & $10^{+0}$ & 7.389056 & $\mathrm{x}$ & $10^{+0}$ & 1.5 & 2.0 & 104 \\
\hline 27 & 7.389056 & $\mathrm{x}$ & $10^{+0}$ & 1.218249 & $\mathrm{x}$ & $10^{+1}$ & 2.0 & 2.5 & 43 \\
\hline 28 & 1.218249 & $\mathrm{x}$ & $10^{+1}$ & 2.008554 & $\mathbf{x}$ & $10^{+1}$ & 2.5 & 3.0 & 13 \\
\hline 29 & 2.008554 & $\mathrm{x}$ & $10^{+1}$ & 3.311545 & $\mathrm{x}$ & $10^{+1}$ & 3.0 & 3.5 & 5 \\
\hline 30 & 3.311545 & $\mathrm{x}$ & $10^{+1}$ & 5.459815 & $\mathrm{x}$ & $10^{+1}$ & 3.5 & 4.0 & 2 \\
\hline 31 & 5.459815 & $\mathrm{x}$ & $10^{+1}$ & 9.001713 & $\mathrm{x}$ & $10^{+1}$ & 4.0 & 4.5 & 3 \\
\hline 32 & 9.001713 & $\mathrm{x}$ & $10^{+1}$ & 1.484132 & $\mathrm{x}$ & $10^{+2}$ & 4.5 & 5.0 & 1 \\
\hline 33 & 1.484132 & $\mathrm{x}$ & $10^{+2}$ & 2.446919 & $\mathrm{x}$ & $10^{+2}$ & 5.0 & 5.5 & 0 \\
\hline 34 & 2.446919 & $\mathrm{x}$ & $10^{+2}$ & 4.034288 & $\mathrm{x}$ & $10^{+2}$ & 5.5 & 6.0 & 0 \\
\hline 35 & 4.034288 & $\mathrm{x}$ & $10^{+2}$ & 6.651416 & $\mathrm{x}$ & $10^{+2}$ & 6.0 & 6.5 & 0 \\
\hline 36 & 6.651416 & $\mathrm{x}$ & $10^{+2}$ & 1.096633 & $\mathrm{x}$ & $10^{+3}$ & 6.5 & 7.0 & 0 \\
\hline 37 & 1.096633 & $\mathrm{x}$ & $10^{+3}$ & 1.808042 & $\mathrm{x}$ & $10^{+3}$ & 7.0 & 7.5 & 1 \\
\hline 38 & 1.808042 & $\mathrm{x}$ & $10^{+3}$ & 2.980958 & $\mathrm{x}$ & $10^{+3}$ & 7.5 & 8.0 & 0 \\
\hline 39 & 2.980958 & $\mathrm{x}$ & $10^{+3}$ & 4.914769 & $\mathrm{x}$ & $10^{+3}$ & 8.0 & 8.5 & 3 \\
\hline
\end{tabular}

Class (C) 
Lower Boundary (LB)

Upper Boundary (UB)

$\log _{\mathrm{e}}$ Lower Boundary (LLB)

$\log _{\mathrm{e}}$ Upper Boundary (LUB)

Class Frequency (CF)

Refer to the $\log _{\mathrm{e}}$ ( variance $_{\mathrm{DGE}} /$ variance $_{\mathrm{SAM}}$ ) ratio frequency distribution (Table 11). The distribution of the $\log _{\mathrm{e}}$ (variance (vGE $_{\mathrm{D}}$ / variance $\mathrm{SAM}_{\mathrm{SM}}$ ) ratios was summarized using the frequency distribution. The frequency distribution classified the $\log _{\mathrm{e}}$ (variance DGE $/$ variance $_{\mathrm{SAM}}$ ) ratios into thirty nine classes. Class twenty two contained 1289 observations which was the largest number of observations in any of the classes. Class twenty two formed the mode of the frequency distribution. The mode included the $\log _{e}$ (variance ${ }_{\mathrm{DGE}} /$ variance $_{\mathrm{SAM}}$ ) ratios falling within the range from the -0.5 normalized fraction lower boundary to the 0.0 normalized fraction upper boundary. The mode included the (variance DGE $_{\text {/ }}$ variance SAM $_{\text {) }}$ ) ratios falling within the range from the 0.60653 normalized fraction lower boundary to the 1.00000 normalized fraction upper boundary. Consider the case of the lower boundary. The mode size of the variance ${ }_{D G E}$ was 0.60653 normalized fraction of the mode size of the variancesAM. The mode size of the variance $_{\text {DGE }}$ was $1.00000-0.60653=0.39347$ normalized fraction smaller than the mode

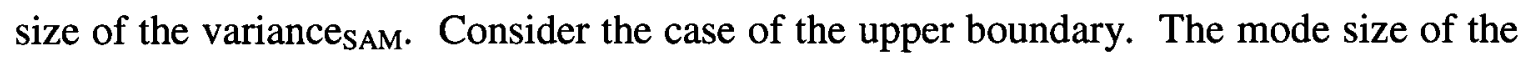
variance $_{\mathrm{DGE}}$ was 1.00000 normalized fraction of the mode size of the variance SAM. The mode size of the variance ${ }_{D G E}$ was $1.00000-1.00000=0.00000$ normalized fraction

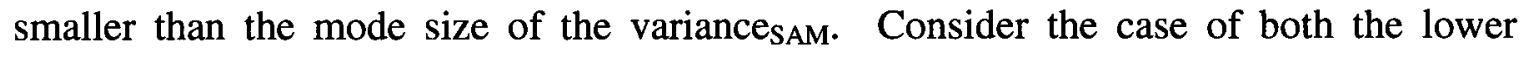
boundary and the upper boundary. The mode size of the variance ${ }_{\mathrm{DGE}}$ was from 0.60653 to 1.00000 normalized fraction of the mode size of the variance ${ }_{\mathrm{SAM}}$. The mode size of the variance $_{\mathrm{DGE}}$ was from 0.39347 to 0.00000 normalized fraction smaller than the mode size of the variance SAM $_{\text {. }}$ 
log ratio of DGE to SAM gene-rank variances

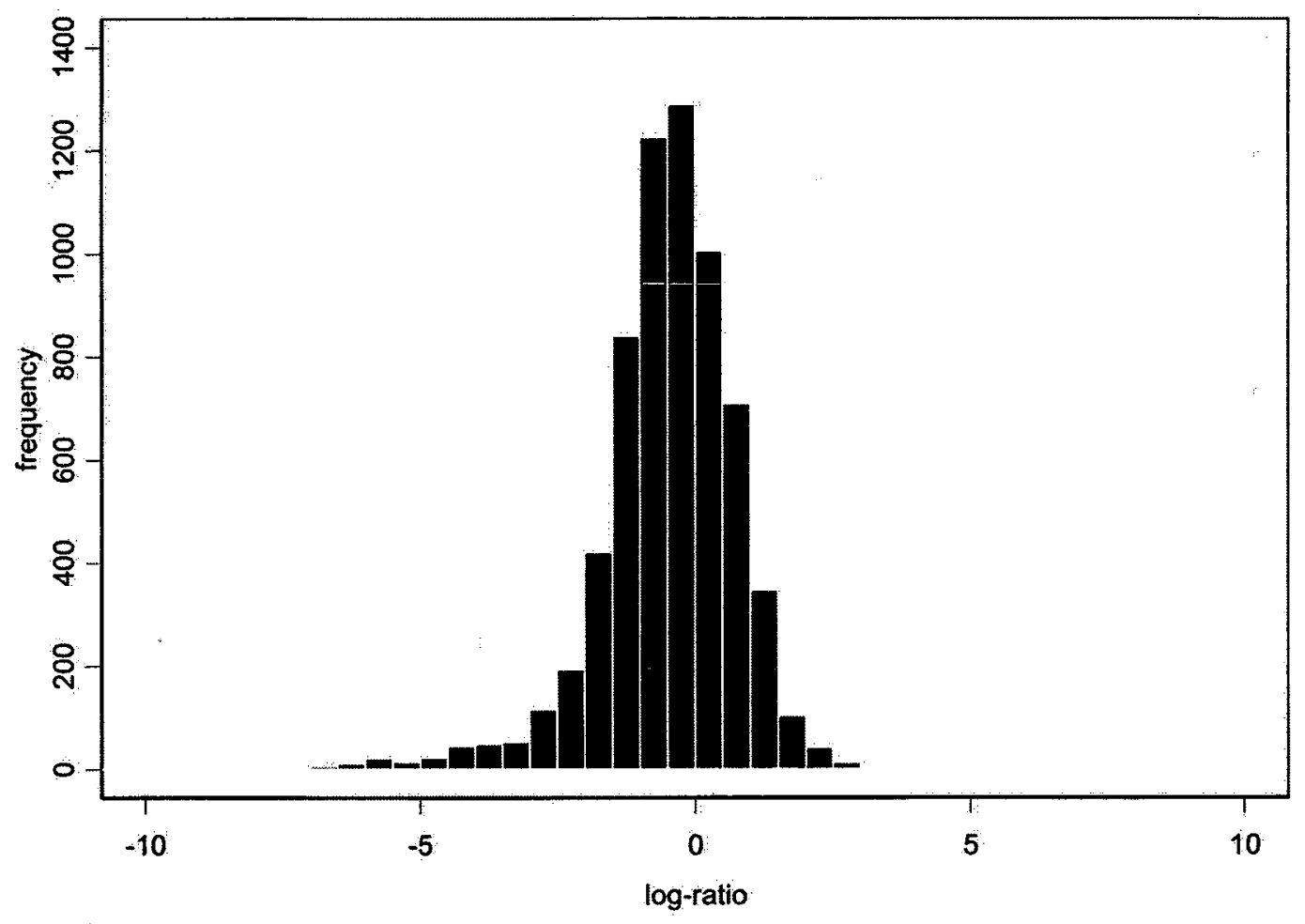

Figure 27. $\log _{\mathrm{e}}\left(\right.$ variance $_{\mathrm{DGE}} /$ variance $\left._{\mathrm{SAM}}\right)$ ratio histogram. 
Refer to the $\log _{\mathrm{e}}$ ( variance $_{\mathrm{DGE}} /$ variance $\mathrm{SAM}$ ) ratio histogram (Figure 27). The ratio frequency distribution was graphically visualized using the ratio histogram. The histogram mode was graphically visualized as the peak which occurred within the negative region of the $\log _{e}$ (variance DGE $_{\text {/ variance }}$ SAM) ratios. The histogram mode occurrence within the negative region of the $\log _{e}$ (variance DGE $_{\text {( variance }}$ SAM) ratios confirmed that the mode size of the variance ${ }_{\text {DGE }}$ was smaller than the mode size of the variance sAM. $_{\text {. }}$

The mean, the median and the mode demonstrated that the variance $_{\mathrm{DGE}}$ was

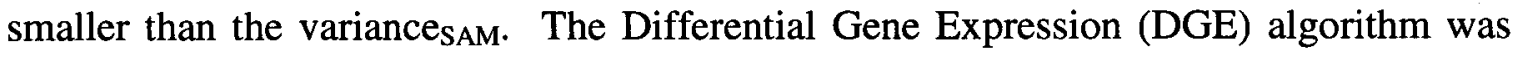
the more precise method than the Significance Analysis of Microarrays (SAM) algorithm. 


\section{Chapter 5 Discussion}

Theoretical models were hypothesized by us for the Significance Analysis of Microarrays (SAM) algorithm and the Differential Gene Expression (DGE) algorithm. The SAM algorithm was based on the use of the modified T-statistic. The modified Tstatistic was referred to as the difference statistic. The difference statistics were used for the statistical gene expression measurements in the SAM algorithm. The difference statistics represented the absolute measurements of the statistical dispersion in the differential computations. The SAM algorithm demonstrated two statistical gene expression measurement properties: 1. nonsymmetric crossover effect and 2 . nonsymmetric differential effect. The DGE algorithm was based on the use of the quantile. The quantiles were used for the statistical gene expression measurements in the DGE algorithm. The quantiles represented the relative measurements of the statistical dispersion in the differential computations. The DGE algorithm demonstrated three statistical gene expression measurement properties: 1. nonsymmetric crossover effect, 2. nonsymmetric differential effect and 3. differential fallback effect. The SAM algorithm used the supervised learning differentiation mechanism for the classification of the statistical gene expression measurements into two categories: 1. nonregulated genes and 2. regulated genes. The DGE algorithm used the unsupervised learning differentiation mechanism for the classification of the statistical gene expression measurements into three categories: 1 . downregulated genes, 2 . nonregulated genes and 3. upregulated genes. The proof-of-concept experiments provided convincing evidence that the SAM algorithm was surpassed by the DGE algorithm which offered five distinct advantages over the SAM algorithm: 1. increased accuracy, 2. increased precision, 3. automated differential criterion selection, 4 . increased statistical gene expression measurement categories and 5. more robust statistical gene expression measurement classification error fault tolerance. The proof-of-concept experiments provided convincing evidence that the SAM algorithm has been superseded by the DGE algorithm as the more efficient alternative comparative datamining algorithm for the solution of the Differential Gene Expression (DGE) problem. Four limitations of the DGE algorithm have been identified: 1. terminal 
quantile computation, 2. terminal expression level measurement computation, 3. local neighborhood computation and 4 . time complexity analysis.

\subsection{Comparative Statistical Gene Expression Measurement Properties}

\subsubsection{Significance Analysis of Microarrays (SAM) Algorithm Statistical Gene Expression Measurement Properties}

Refer to the Significance Analysis of Microarrays (SAM) algorithm difference statistic graph and expected difference statistic graph (Figure 5). The SAM algorithm difference statistic graph and expected difference statistic graph was a composite graph that consisted of two component graphs: 1 . difference statistic versus difference statistic graph and 2. expected difference statistic versus difference statistic graph.

The difference statistic versus difference statistic graph plotted the difference statistics on both the abscissa axis and the ordinate axis. The difference statistic versus difference statistic graph formed the diagonal line. The expected difference statistic versus difference statistic graph plotted the difference statistics on the abscissa axis and the expected difference statistics on the ordinate axis. The differentials were computed as the absolute differences between the difference statistics and the expected difference statistics: for $\mathrm{i}=1$ to $n, d_{i}=a b s\left(E\left(s_{i}\right)-s_{i}\right)$. The differential computations were analyzed for three statistical gene expression measurement properties: 1. nonsymmetric crossover effect, 2. nonsymmetric differential effect and 3. differential fallback effect.

The nonsymmetric crossover effect involved the intersection point between the two graphs. The intersection point between the two graphs corresponded to the minimum absolute differential ordered pair $\left(\mathrm{s}_{\mathrm{ma}}, \mathrm{E}\left(\mathrm{s}_{\mathrm{ma}}\right)\right.$ ). The minimum absolute differential difference statistic $\mathrm{s}_{\mathrm{ma}}$ was not equal to the midpoint difference statistic $\mathrm{s}_{(\mathrm{n} / 2)}, \mathrm{s}_{\mathrm{ma}} \neq \mathrm{s}_{(\mathrm{n} / 2)}$. The intersection point between the two graphs exhibited the nonsymmetric crossover effect. 
The nonsymmetric differential effect involved the initial differential $d_{1}$ and the final differential $d_{n}$. The initial differential $d_{1}$ was not equal in size to the final differential $d_{n}, d_{1} \neq d_{n}$. The initial differential $d_{1}$ and the final differential $d_{n}$ exhibited the nonsymmetric differential effect.

The differential fallback effect involved the differentials. The intersection point between the two graphs corresponded to the minimum absolute differential ordered pair $\left(\mathrm{s}_{\mathrm{ma}}, \mathrm{E}\left(\mathrm{s}_{\mathrm{ma}}\right)\right)$. When decreasing the difference statistics from the minimum absolute differential difference statistic $s_{\mathrm{ma}}$ towards the initial difference statistic $\mathrm{s}_{1}$, the difference statistics and the expected difference statistics underwent divergence. The differentials increased in size. When increasing the difference statistics from the minimum absolute differential difference statistic $s_{m a}$ towards the final difference statistic $s_{n}$, the difference statistics and the expected difference statistics underwent divergence. The differentials increased in size. The initial differential $d_{1}$ and the final differential $d_{n}$ were the largest differentials in size. The differentials did not exhibit the differential fallback effect.

The theoretical basis for the nonsymmetric crossover effect was hypothesized. The nonsymmetric crossover effect was hypothesized to originate when the downregulated difference statistics and the upregulated difference statistics were not equal. The theoretical basis for the nonsymmetric crossover effect remains unknown.

The theoretical basis for the nonsymmetric differential effect was hypothesized. The differentials were computed as the absolute differences between the difference statistics and the expected difference statistics: for $i=1$ to $n, d_{i}=a b s\left(E\left(s_{i}\right)-s_{i}\right)$. The expected difference statistics were computed as the average difference statistics arising from the $C(6,3)=20$ combinations of the six microarray datasets consisting of the three control microarray datasets and the three experimental microarray datasets. The initial differential $d_{1}$ was the global absolute quantitative measurement of the downregulated difference statistics. The final differential $d_{n}$ was the global absolute quantitative measurement of the upregulated difference statistics. The nonsymmetric differential effect was hypothesized to originate when the downregulated difference statistics and the 
upregulated difference statistics were not equal, $d_{1} \neq d_{n}$. The theoretical basis for the nonsymmetric differential effect remains unknown.

The theoretical basis for the absence of the differential fallback effect was hypothesized. The statistical gene expression measurements were based on the difference statistics. The differentials were computed as the absolute differences between the difference statistics and the expected difference statistics: for $i=1$ to $n, d_{i}=a b s\left(E\left(s_{i}\right)\right.$ $\mathrm{s}_{\mathrm{i}}$ ). The expected difference statistics were computed as the average difference statistics arising from the $C(6,3)=20$ combinations of the six microarray datasets consisting of the three control microarray datasets and the three experimental microarray datasets. The absence of the differential fallback effect was hypothesized to originate from the use of the difference statistics in the differential computations because the difference statistics represented the absolute measurements of the statistical dispersion. The absolute measurement nature of the difference statistics meant that the deviations of the difference statistics from the minimum absolute differential difference statistic $s_{\mathrm{ma}}$ resulted in unconstrained increases in the differentials. The theoretical basis for the absence of the differential fallback effect remains unknown.

The SAM algorithm demonstrated the nonsymmetric crossover effect and the nonsymmetric differential effect. The SAM algorithm did not demonstrate the differential fallback effect.

\subsubsection{Differential Gene Expression (DGE) Algorithm Statistical Gene Expression Measurement Properties}

Refer to the Differential Gene Expression (DGE) algorithm quantile graph and expected quantile graph (Figure 9). The DGE algorithm quantile graph and expected quantile graph was a composite graph that consisted of two component graphs: 1 . quantile versus quantile graph and 2. expected quantile versus quantile graph. 
The quantile versus quantile graph plotted the quantiles on both the abscissa axis and the ordinate axis. The quantile versus quantile graph formed the diagonal line. The expected quantile versus quantile graph plotted the quantiles on the abscissa axis and the expected quantiles on the ordinate axis. The differentials were computed as the absolute differences between the quantiles and the expected quantiles: for $i=1$ to $n, d_{i}=a b s(E$ $\left.\left(\mathrm{q}_{\mathrm{i}}\right)-\mathrm{q}_{\mathrm{i}}\right)$. The differential computations were analyzed for three statistical gene expression measurement properties: 1. nonsymmetric crossover effect, 2. nonsymmetric differential effect and 3. differential fallback effect.

The nonsymmetric crossover effect involved the intersection point between the two graphs. The intersection point between the two graphs corresponded to the minimum absolute differential ordered pair $\left(\mathrm{q}_{\mathrm{ma}}, \mathrm{E}\left(\mathrm{q}_{\mathrm{ma}}\right)\right)$. The minimum absolute differential quantile $\mathrm{q}_{\mathrm{ma}}$ was not equal to the midpoint quantile $\mathrm{q}_{(\mathrm{n} / 2)}, \mathrm{q}_{\mathrm{ma}} \neq \mathrm{q}_{(\mathrm{n} / 2)}$. The intersection point between the two graphs exhibited the nonsymmetric crossover effect.

The nonsymmetric differential effect involved the initial differential $d_{1}$ and the final differential $d_{n}$. The initial differential $d_{1}$ was not equal in size to the final differential $d_{n}, d_{1} \neq d_{n}$. The initial differential $d_{1}$ and the final differential $d_{n}$ exhibited the nonsymmetric differential effect.

The differential fallback effect involved the differentials. The intersection point between the two graphs corresponded to the minimum absolute differential ordered pair $\left(\mathrm{q}_{\mathrm{ma}}, \mathrm{E}\left(\mathrm{q}_{\mathrm{ma}}\right)\right.$ ). When decreasing the quantiles from the minimum absolute differential quantile $\mathrm{q}_{\mathrm{ma}}$ towards the maximum positive differential quantile $\mathrm{q}_{\mathrm{mp}}$, the quantiles and the expected quantiles underwent divergence. The differentials increased in size. Upon reaching the maximum positive differential quantile $\mathrm{q}_{\mathrm{mp}}$, the quantiles and the expected quantiles ceased divergence. The differentials reached the maximum positive size. The maximum positive differential ordered pair $\left(q_{m p}, E\left(q_{m p}\right)\right)$ formed the first inflection point in the expected quantile versus quantile graph. When decreasing the quantiles from the maximum positive differential quantile $\mathrm{q}_{\mathrm{mp}}$ towards the initial quantile $\mathrm{q}_{1}$, the quantiles and the expected quantiles underwent convergence. The differentials decreased in size. 
When decreasing the quantiles from the minimum absolute differential quantile $\mathrm{q}_{\mathrm{ma}}$ towards the initial quantile $q_{1}$, the expected quantile versus quantile graph formed the downregulation biphasic curve. When increasing the quantiles from the minimum absolute differential quantile $\mathrm{q}_{\mathrm{ma}}$ towards the maximum negative differential quantile $\mathrm{q}_{\mathrm{mn}}$, the quantiles and the expected quantiles underwent divergence. The differentials increased in size. Upon reaching the maximum negative differential quantile $\mathrm{q}_{\mathrm{mn}}$, the quantiles and the expected quantiles ceased divergence. The differentials reached the maximum negative size. The maximum negative differential ordered pair $\left(\mathrm{q}_{\mathrm{mn}}, \mathrm{E}\left(\mathrm{q}_{\mathrm{mn}}\right)\right)$ formed the second inflection point in the expected quantile versus quantile graph. When increasing the quantiles from the maximum negative differential quantile $\mathrm{q}_{\mathrm{mn}}$ towards the final quantile $\mathrm{q}_{\mathrm{n}}$, the quantiles and the expected quantiles underwent convergence. The differentials decreased in size. When increasing the quantiles from the minimum absolute differential quantile $q_{m a}$ towards the final quantile $q_{n}$, the expected quantile versus quantile graph formed the upregulation biphasic curve. In total, the expected quantile versus quantile graph formed two biphasic curves. The initial differential $d_{1}$ and the final differential $d_{n}$ were not the largest differentials in size. The differentials exhibited the differential fallback effect.

The theoretical basis for the nonsymmetric crossover effect was hypothesized. The nonsymmetric crossover effect was hypothesized to originate when the downregulated quantiles and the upregulated quantiles were not equal. The theoretical basis for the nonsymmetric crossover effect remains unknown.

The theoretical basis for the nonsymmetric differential effect was hypothesized. The differentials were computed as the absolute differences between the quantiles and the expected quantiles: for $\mathrm{i}=1$ to $\mathrm{n}, \mathrm{d}_{\mathrm{i}}=$ abs $\left(E\left(\mathrm{q}_{\mathrm{i}}\right)-\mathrm{q}_{\mathrm{i}}\right)$. The expected quantiles were computed as the average quantiles arising from the $C(6,3)=20$ combinations of the six microarray datasets consisting of the three control microarray datasets and the three experimental microarray datasets. The maximum positive differential $\mathrm{d}_{\mathrm{mp}}$ was the global absolute quantitative measurement of the downregulated quantiles. The maximum negative differential $d_{m n}$ was the global absolute quantitative measurement of the 
upregulated quantiles. The nonsymmetric differential effect was hypothesized to originate when the downregulated quantiles and the upregulated quantiles were not equal, $\mathrm{d}_{\mathrm{mp}} \neq \mathrm{d}_{\mathrm{mn}}$.

The global absolute quantitative measurement of the downregulated quantiles and the global absolute quantitative measurement of the upregulated quantiles were compared on the relative basis using two inverse statistics: 1. downregulated / upregulated quantile ratio and 2. upregulated / downregulated quantile ratio. The downregulated / upregulated quantile ratio (DUQR) was the global relative quantitative measurement of the downregulated quantiles. The DUQR was defined as DUQR $=d_{m p} / d_{m n}$. The upregulated / downregulated quantile ratio (UDQR) was the global relative quantitative measurement of the upregulated quantiles. The UDQR was defined as UDQR $=d_{m n}$ I $d_{m p}$. The theoretical basis for the nonsymmetric differential effect remains unknown.

The theoretical basis for the differential fallback effect was hypothesized. The statistical gene expression measurements were based on the quantiles. The differentials were computed as the absolute differences between the quantiles and the expected quantiles: for $\mathrm{i}=1$ to $n, \mathrm{~d}_{\mathrm{i}}=\mathrm{abs}\left(\mathrm{E}\left(\mathrm{q}_{\mathrm{i}}\right)-\mathrm{q}_{\mathrm{i}}\right)$. The expected quantiles were computed as the average quantiles arising from the $\mathrm{C}(6,3)=20$ combinations of the six microarray datasets consisting of the three control microarray datasets and the three experimental microarray datasets. The expected quantile versus quantile graph formed two biphasic curves: 1 . downregulation biphasic curve and 2. upregulation biphasic curve.

The downregulation biphasic curve occurred in the expected quantile versus quantile graph when decreasing the quantiles from the minimum absolute differential quantile $\mathrm{q}_{\mathrm{ma}}$ towards the initial quantile $\mathrm{q}_{1}$. The downregulation biphasic curve was partitioned into five quantile intervals: 1. $\mathrm{q}_{\mathrm{ma}}, 2 . \mathrm{q}_{\mathrm{mp}}<\mathrm{q}<\mathrm{q}_{\mathrm{ma}}, 3 . \mathrm{q}_{\mathrm{mp}}, 4 . \mathrm{q}_{1}<\mathrm{q}<\mathrm{q}_{\mathrm{mp}}$ and 5. $\mathrm{q}_{1}$.

The first quantile interval consisted of the minimum absolute differential quantile $\mathrm{q}_{\mathrm{ma}}$. The minimum absolute differential ordered pair $\left(\mathrm{q}_{\mathrm{ma}}, \mathrm{E}\left(\mathrm{q}_{\mathrm{ma}}\right)\right.$ ) formed the initial 
endpoint of the downregulation biphasic curve. The minimum absolute differential quantile $\mathrm{q}_{\mathrm{ma}}$ caused the approximately zero divergence between the quantiles and the expected quantiles. The static divergence between the quantiles and the expected quantiles resulted in the static minimum absolute differential $\mathrm{d}_{\text {ma. }}$.

The second quantile interval consisted of the quantiles between the minimum absolute differential quantile $\mathrm{q}_{\mathrm{ma}}$ and the maximum positive differential quantile $\mathrm{q}_{\mathrm{mp}}$. Decreasing the quantiles from the minimum absolute differential quantile $\mathrm{q}_{\mathrm{ma}}$ towards the maximum positive differential quantile $\mathrm{q}_{\mathrm{mp}}$ caused the expected quantiles to decrease at the slower rate than the quantiles. The increasing divergence between the quantiles and the expected quantiles resulted in the increasing positive differentials.

The third quantile interval consisted of the maximum positive differential quantile $\mathrm{q}_{\mathrm{mp}}$. The maximum positive differential ordered pair $\left(\mathrm{q}_{\mathrm{mp}}, \mathrm{E}\left(\mathrm{q}_{\mathrm{mp}}\right)\right)$ formed the inflection point of the downregulation biphasic curve. The maximum positive differential quantile $\mathrm{q}_{\mathrm{mp}}$ caused the maximum positive divergence between the quantiles and the expected quantiles. The maximum positive differential quantile $q_{m p}$ caused the expected quantiles to decrease at the same rate as the quantiles. The static divergence between the quantiles and the expected quantiles resulted in the static maximum positive differential $\mathrm{d}_{\mathrm{mp}}$.

The fourth quantile interval consisted of the quantiles between the maximum positive differential quantile $q_{m p}$ and the initial quantile $q_{1}$. Decreasing the quantiles from the maximum positive differential quantile $\mathrm{q}_{\mathrm{mp}}$ towards the initial quantile $\mathrm{q}_{1}$ caused the expected quantiles to decrease at the faster rate than the quantiles. The decreasing divergence between the quantiles and the expected quantiles resulted in the decreasing positive differentials.

The fifth quantile interval consisted of the initial quantile $\mathrm{q}_{1}$. The initial quantile ordered pair $\left(\mathrm{q}_{1}, \mathrm{E}\left(\mathrm{q}_{1}\right)\right)$ formed the final endpoint of the downregulation biphasic curve. The initial quantile $\mathrm{q}_{1}$ caused the minor positive divergence between the quantiles and the 
expected quantiles. The static divergence between the quantiles and the expected quantiles resulted in the static initial differential $d_{1}$.

The second quantile interval was marked by the expected quantiles slow decrease. The second quantile interval was driven by the expected quantiles slow decrease process. The third quantile interval was marked by the acceleration transition point between the expected quantiles slow decrease and the expected quantiles fast decrease. The maximum positive differential quantile $\mathrm{q}_{\mathrm{mp}}$ was the global absolute qualitative measurement of the downregulated quantiles. The fourth quantile interval was marked by the expected quantiles fast decrease. The fourth quantile interval was driven by the expected quantiles fast decrease process. The downregulation biphasic curve resulted from the competition between the two opposite processes: 1. expected quantiles slow decrease process and 2. expected quantiles fast decrease process. The downregulation biphasic curve was the first example of the differential fallback effect.

The upregulation biphasic curve occurred in the expected quantile versus quantile graph when increasing the quantiles from the minimum absolute differential quantile $q_{m a}$ towards the final quantile $\mathrm{q}_{\mathrm{n}}$. The upregulation biphasic curve was partitioned into five quantile intervals: 1. $\mathrm{q}_{\mathrm{ma}}, 2 . \mathrm{q}_{\mathrm{ma}}<\mathrm{q}<\mathrm{q}_{\mathrm{mn}}, 3 . \mathrm{q}_{\mathrm{mn}}, 4 . \mathrm{q}_{\mathrm{mn}}<\mathrm{q}<\mathrm{q}_{\mathrm{n}}$ and 5. $\mathrm{q}_{\mathrm{n}}$.

The first quantile interval consisted of the minimum absolute differential quantile $\mathrm{q}_{\mathrm{ma}}$. The minimum absolute differential ordered pair $\left(\mathrm{q}_{\mathrm{ma}}, \mathrm{E}\left(\mathrm{q}_{\mathrm{ma}}\right)\right.$ ) formed the initial endpoint of the upregulation biphasic curve. The minimum absolute differential quantile $\mathrm{q}_{\mathrm{ma}}$ caused the approximately zero divergence between the quantiles and the expected quantiles. The static divergence between the quantiles and the expected quantiles resulted in the static minimum absolute differential $\mathrm{d}_{\mathrm{ma}}$.

The second quantile interval consisted of the quantiles between the minimum absolute differential quantile $q_{m a}$ and the maximum negative differential quantile $q_{m n}$. Increasing the quantiles from the minimum absolute differential quantile $\mathrm{q}_{\mathrm{ma}}$ towards the maximum negative differential quantile $\mathrm{q}_{\mathrm{mn}}$ caused the expected quantiles to increase at 
the slower rate than the quantiles. The increasing divergence between the quantiles and the expected quantiles resulted in the increasing negative differentials.

The third quantile interval consisted of the maximum negative differential quantile $\mathrm{q}_{\mathrm{mn}}$. The maximum negative differential ordered pair $\left(\mathrm{q}_{\mathrm{mn}}, \mathrm{E}\left(\mathrm{q}_{\mathrm{mn}}\right)\right)$ formed the inflection point of the upregulation biphasic curve. The maximum negative differential quantile $\mathrm{q}_{\mathrm{mn}}$ caused the maximum negative divergence between the quantiles and the expected quantiles. The maximum negative differential quantile $\mathrm{q}_{\mathrm{mn}}$ caused the expected quantiles to increase at the same rate as the quantiles. The static divergence between the quantiles and the expected quantiles resulted in the static maximum negative differential $\mathrm{d}_{\mathrm{mn}}$.

The fourth quantile interval consisted of the quantiles between the maximum negative differential quantile $\mathrm{q}_{\mathrm{mn}}$ and the final quantile $\mathrm{q}_{\mathrm{n}}$. Increasing the quantiles from the maximum negative differential quantile $\mathrm{q}_{\mathrm{mn}}$ towards the final quantile $\mathrm{q}_{\mathrm{n}}$ caused the expected quantiles to increase at the faster rate than the quantiles. The decreasing divergence between the quantiles and the expected quantiles resulted in the decreasing negative differentials.

The fifth quantile interval consisted of the final quantile $q_{n}$. The final quantile ordered pair $\left(q_{n}, E\left(q_{n}\right)\right)$ formed the final endpoint of the upregulation biphasic curve. The final quantile $q_{n}$ caused the minor negative divergence between the quantiles and the expected quantiles. The static divergence between the quantiles and the expected quantiles resulted in the static final differential $d_{n}$.

The second quantile interval was marked by the expected quantiles slow increase. The second quantile interval was driven by the expected quantiles slow increase process. The third quantile interval was marked by the acceleration transition point between the expected quantiles slow increase and the expected quantiles fast increase. The maximum negative differential quantile $\mathrm{q}_{\mathrm{mn}}$ was the global absolute qualitative measurement of the upegulated quantiles. The fourth quantile interval was marked by the expected quantiles 
fast increase. The fourth quantile interval was driven by the expected quantiles fast increase process. The upregulation biphasic curve resulted from the competition between the two opposite processes: 1 . expected quantiles slow increase process and 2. expected quantiles fast increase process. The upregulation biphasic curve was the second example of the differential fallback effect.

The differential fallback effect was hypothesized to originate from the use of the quantiles in the differential computations because the quantiles represented the relative measurements of the statistical dispersion. The relative measurement nature of the quantiles meant that the deviations of the quantiles from the minimum absolute differential quantile $\mathrm{q}_{\mathrm{ma}}$ resulted in constrained increases in the differentials. The theoretical basis for the differential fallback effect remains unknown.

The DGE algorithm demonstrated the nonsymmetric crossover effect, the nonsymmetric differential effect and the differential fallback effect.

\subsection{Comparative Differentiation Mechanisms}

\subsubsection{Significance Analysis of Microarrays (SAM) Algorithm Differentiation Mechanism}

The Significance Analysis of Microarrays (SAM) algorithm used the supervised learning differentiation mechanism for the classification of the statistical gene expression measurements into the different categories. The statistical gene expression measurements were based on difference statistics. The difference statistics were sorted on the basis of ascending differential order. The one differential criterion was chosen by taking advantage of the human consultation through the utilization of the total discovery rate (TDR) graph and the false discovery rate (FDR) graph. Human intervention was necessary. The one differential criterion was used directly as the decision factor for the classification of the differentials into two statistical gene expression measurement categories: 1. nonregulated genes and 2. regulated genes. The differential classifications 
were restricted to only two statistical gene expression measurement categories: 1 . nonregulated genes and 2. regulated genes. The statistical gene expression measurement categories did not differentiate between the downregulated genes and the upregulated genes. The differential classifications were sensitive to the statistical gene expression measurement classification errors caused by two statistical gene expression measurement properties: 1 . nonsymmetric crossover effect and 2 . nonsymmetric differential effect.

\subsubsection{Differential Gene Expression (DGE) Algorithm Differentiation Mechanism}

The Differential Gene Expression (DGE) algorithm used the unsupervised learning differentiation mechanism for the classification of the statistical gene expression measurements into the different categories. The statistical gene expression measurements were based on quantiles. The quantiles were sorted on the basis of ascending quantile order. The two differential criteria were chosen by taking advantage of the differential fallback effect through the utilization of the differential maximum inclusion method. Human intervention was not necessary. The two differential criteria corresponded to the maximum positive differential $d_{m p}$ and the maximum negative differential $d_{m n}$. The maximum positive differential $d_{m p}$ was used indirectly for the selection of the maximum positive differential quantile $q_{m p}$. The maximum negative differential $d_{m n}$ was used indirectly for the selection of the maximum negative differential quantile $q_{\mathrm{mn}}$. The maximum positive differential quantile $\mathrm{q}_{\mathrm{mp}}$ and the maximum negative differential quantile $\mathrm{q}_{\mathrm{mn}}$ were used directly as the decision factors for the classification of the quantiles into three statistical gene expression measurement categories: 1 . downregulated genes, 2. nonregulated genes and 3. upregulated genes. The quantile classifications were expanded to include three statistical gene expression measurement categories: 1 . downregulated genes, 2. nonregulated genes and 3. upregulated genes. The statistical gene expression measurement categories differentiated between the downregulated genes and the upregulated genes. The quantile classifications were resistant to the statistical gene expression measurement classification errors caused by two statistical gene expression measurement properties: 1. nonsymmetric crossover effect and 2 . nonsymmetric differential effect. 


\subsection{Comparative Analysis}

The proof-of-concept experiments provided convincing evidence that the Significance Analysis of Microarrays (SAM) algorithm was surpassed by the Differential Gene Expression (DGE) algorithm which offered five distinct advantages over the SAM algorithm: 1. accuracy performance analysis, 2. precision performance analysis, 3 . differential criterion selection, 4. statistical gene expression measurement categories and 5. statistical gene expression measurement classification error fault tolerance.

The accuracy performance analysis involved the degree of conformity of the actual differentially-expressed genes and the predicted differentially-expressed genes. The SAM algorithm demonstrated smaller accuracy performance. The DGE algorithm demonstrated larger accuracy performance. The DGE algorithm offered increased accuracy.

The precision performance analysis involved the degree of consistency of the differential ranks with themselves. The SAM algorithm demonstrated smaller precision performance. The DGE algorithm demonstrated larger precision performance. The DGE algorithm offered increased precision.

The differential criterion selection involved the method used for the choice of the differential criterion. The SAM algorithm used the human intervention method for the choice of the one differential criterion. The SAM algorithm engaged in a form of supervised learning. The DGE algorithm did not use the human intervention method for the choice of the two differential criteria. The DGE algorithm engaged in a form of unsupervised learning. The DGE algorithm offered automated differential criterion selection.

The statistical gene expression measurement categories involved the classification of the statistical gene expression measurements into the different categories. The SAM 
algorithm classified the statistical gene expression measurements into two categories: 1 . nonregulated genes and 2. regulated genes. The SAM algorithm statistical gene expression measurement categories did not differentiate between the downregulated genes and the upregulated genes. The DGE algorithm classified the statistical gene expression measurements into three categories: 1 . downregulated genes, 2 . nonregulated genes and 3. upregulated genes. The DGE algorithm statistical gene expression measurement categories differentiated between the downregulated genes and the upregulated genes. The DGE algorithm offered an increased number of more detailed statistical gene expression measurement categories.

The statistical gene expression measurement classification error fault tolerance involved the fault tolerance to the statistical gene expression measurement classification errors caused by two statistical gene expression measurement properties: 1 . nonsymmetric crossover effect and 2. nonsymmetric differential effect. The SAM algorithm was less fault tolerant to the statistical gene expression measurement classification errors. The DGE algorithm was more fault tolerant to the statistical gene expression measurement classification errors. The DGE algorithm offered more robust statistical gene expression measurement classification error fault tolerance.

The proof-of-concept experiments provided convincing evidence that the SAM algorithm has been superseded by the DGE algorithm as the more efficient alternative comparative datamining algorithm for the solution of the Differential Gene Expression (DGE) problem.

\subsection{Differential Gene Expression (DGE) Algorithm Limitations}

The Differential Gene Expression (DGE) algorithm demonstrated four computational limitations: 1. terminal quantile computation, 2. terminal expression level measurement computation, 3 . local neighborhood computation and 4. time complexity analysis. 
The terminal quantiles consisted of two sets of quantiles: 1. initial quantiles and 2. final quantiles. The initial quantiles included the quantiles which were lesser than the 0.01 quantile, $\mathrm{q}_{\text {initial }}<0.01$. The final quantiles included the quantiles which were greater than the 0.99 quantile, $0.99<\mathrm{q}_{\text {final }}$. The terminal quantiles occurred under the conditions of the sparse datapoint densities. The terminal quantiles could not be accurately computed using the nonparametric quantile regression algorithm which required the conditions of the dense datapoint densities (Yu and Jones 1998). The nonparametric quantile regression algorithm requires extension which would enable the computation of the terminal quantiles under the conditions of the sparse datapoint densities (Yu and Jones 1998).

The terminal expression level measurements consisted of two sets of expression level measurements: 1. initial expression level measurements and 2. final expression level measurements. The initial expression level measurements consisted of the smallest $x$ values in the input data. The final expression level measurements consisted of the largest $\mathrm{X}$ values in the input data. The terminal expression level measurements occurred under the conditions of the sparse datapoint densities. The terminal expression level measurements could not be accurately computed using the nonparametric quantile regression algorithm which required the conditions of the dense datapoint densities ( $\mathrm{Yu}$ and Jones 1998). The nonparametric quantile regression algorithm requires extension which would enable the computation of the terminal expression level measurements under the conditions of the sparse datapoint densities (Yu and Jones 1998).

The local neighborhoods defined the immediate vicinities of the quantiles. The immediate vicinities of the quantiles identified the local datapoints which were used as the input for the computation of the quantiles. The local neighborhood algorithm has not been subjected to rigorous optimization for the determination of the optimal local neighborhood sizes (Yu and Jones 1998). The local neighborhood algorithm requires extension which would enable the computation of the optimal local neighborhood sizes (Yu and Jones 1998). 
The time complexity analysis revealed that the quantile computations were the slowest rate-limiting step of the DGE algorithm (Yu and Jones 1969). Since the quantile computations were mutually independent, the quantiles could be more rapidly computed using the parallel computer implementation. Greater accuracy could be achieved by increasing the number of grid points and the number of quantiles. However, increasing the number of grid points and the number of quantiles would result in additional computational time requirements. Under the conditions of the increased number of grid points and the increased number of quantiles, the parallel computer implementation would generate further computational time reductions. The quantile computations require the parallel computer implementation.

The DGE algorithm would be enhanced through the incorporation of the extended nonparametric quantile regression algorithm capable of the computation of the terminal quantiles under the conditions of the sparse datapoint densities ( $\mathrm{Yu}$ and Jones 1998). The DGE algorithm would be enhanced through the incorporation of the extended nonparametric quantile regression algorithm capable of the computation of the terminal expression level measurements under the conditions of the sparse datapoint densities ( $\mathrm{Yu}$ and Jones 1998). The DGE algorithm would be enhanced through the incorporation of the extended local neighborhood algorithm capable of the computation of the optimal local neighborhood sizes. The DGE algorithm would be enhanced through the parallel computer implementation capable of the rapid quantile computations. The DGE algorithm would then demonstrate further predicted improvements in four advantages: 1 . accuracy performance analysis, 2. precision performance analysis, 3. statistical gene expression measurement classification error fault tolerance and 4. computational time reductions. 


\section{Chapter 6 Conclusions}

The doctoral thesis successfully accomplished the defined objective in which the novel Differential Gene Expression (DGE) algorithm was developed as the more efficient alternative comparative datamining algorithm for the solution of the Differential Gene Expression (DGE) problem. The novel normalization algorithm was developed as a prerequisite to allowing the meaningful comparative microarray analysis between the control microarray datasets and the experimental microarray datasets in the differential gene expression algorithms. The DGE algorithm was based on the Nonlinear FoldChange (NFC) method which was based in turn on the nonparametric quantile regression analysis. The DGE algorithm was based on the use of quantiles which demonstrated three statistical gene expression measurement properties: 1. nonsymmetric crossover effect, 2. nonsymmetric differential effect and 3. differential fallback effect. The DGE algorithm differentiation mechanism took advantage of the differential fallback effect through the utilization of the differential maximum inclusion method for the classification of the statistical gene expression measurements into three categories: 1 . downregulated genes, 2 . nonregulated genes and 3. upregulated genes.

The proof-of-concept experiments provided convincing evidence that the Significance Analysis of Microarrays (SAM) algorithm was surpassed by the DGE algorithm which offered five distinct advantages over the SAM algorithm: 1. increased accuracy, 2. increased precision, 3. automated differential criterion selection, 4 . increased statistical gene expression measurement categories and 5. more robust statistical gene expression measurement classification error fault tolerance. The proof-of-concept experiments provided convincing evidence that the SAM algorithm has been superseded by the DGE algorithm as the more efficient alternative comparative datamining algorithm for the solution of the DGE problem. Four limitations of the DGE algorithm have been identified: 1. terminal quantile computation, 2. terminal expression level measurement computation, 3 . local neighborhood computation and 4. time complexity analysis. The DGE algorithm will advance six fields of contemporary scientific research: 1. molecular target identification, 2. stem cell development, 3. toxicogenomics and radiation 
biophysics, 4. complex disease gene mapping, 5. diagnostic biomarkers and 6 . pharmacogenomics. Five future research directions for the DGE algorithm have been proposed: 1. statistical gene expression measurement properties, 2. extended nonparametric quantile regression algorithm, 3. extended local neighborhood algorithm, 4. parallel computer implementation and 5. receiver operating characteristic (ROC) curves.

\subsection{Differential Gene Expression (DGE) Algorithm Summary}

\subsubsection{Differential Gene Expression (DGE) Algorithm}

The novel Differential Gene Expression (DGE) algorithm was developed as the more efficient alternative comparative datamining algorithm for the solution of the Differential Gene Expression (DGE) problem. The novel normalization algorithm formed the preliminary step of the DGE algorithm. The normalization algorithm was developed for the standardization of the signals in the control state microarray datasets and the experimental state microarray datasets to comparable levels of intensity. Transformed microarray datasets compensated for the systematic variations as a prerequisite to allowing the meaningful comparative microarray analysis between the control microarray datasets and the experimental microarray datasets in the differential gene expression algorithms. The DGE algorithm was based on the Nonlinear FoldChange (NFC) method. The NFC method was based on the nonparametric quantile regression analysis. The nonparametric quantile regression analysis was implemented using the kernel weighted local linear fitting algorithm to approximate conditional quantile functions ( $\mathrm{Yu}$ and Jones 1998).

The DGE algorithm was based on the use of quantiles as the statistical gene expression measurements. The quantiles demonstrated three statistical gene expression measurement properties: 1 . nonsymmetric crossover effect, 2 . nonsymmetric differential effect and 3. differential fallback effect. 
The DGE algorithm used the following differentiation mechanism for the classification of the statistical gene expression measurements into the different categories. The statistical gene expression measurements were based on quantiles. The quantiles were sorted on the basis of ascending quantile order. The two differential criteria were chosen by taking advantage of the differential fallback effect through the utilization of the differential maximum inclusion method. Human intervention was not necessary. The two differential criteria corresponded to the maximum positive differential $d_{m p}$ and the maximum negative differential $d_{m n}$. The maximum positive differential $d_{m p}$ was used indirectly for the selection of the maximum positive differential quantile $q_{m p}$. The maximum negative differential $d_{m n}$ was used indirectly for the selection of the maximum negative differential quantile $\mathrm{q}_{\mathrm{mn}}$. The maximum positive differential quantile $\mathrm{q}_{\mathrm{mp}}$ and the maximum negative differential quantile $\mathrm{q}_{\mathrm{mn}}$ were used directly as the decision factors for the classification of the quantiles into three statistical gene expression measurement categories: 1. downregulated genes, 2. nonregulated genes and 3. upregulated genes. The quantile classifications were expanded to include three statistical gene expression measurement categories: 1. downregulated genes, 2. nonregulated genes and 3. upregulated genes. The statistical gene expression measurement categories differentiated between the downregulated genes and the upregulated genes. The quantile classifications were resistant to the statistical gene expression measurement classification errors caused by two statistical gene expression measurement properties: 1 . nonsymmetric crossover effect and 2. nonsymmetric differential effect.

\subsubsection{Comparative Analysis}

The proof-of-concept experiments provided convincing evidence that the Significance Analysis of Microarrays (SAM) algorithm was surpassed by the Differential Gene Expression (DGE) algorithm which offered five distinct advantages over the SAM algorithm: 1. accuracy performance analysis, 2. precision performance analysis, 3 . differential criterion selection, 4 . statistical gene expression measurement categories and 5. statistical gene expression measurement classification error fault tolerance. The DGE 
algorithm offered increased accuracy, increased precision and employed an automated differential criterion selection. The DGE algorithm provided an increased number of more detailed statistical gene expression measurement categories. The DGE algorithm demonstrated more robust statistical gene expression measurement classification error fault tolerance. The proof-of-concept experiments provided convincing evidence that the SAM algorithm has been superseded by the DGE algorithm as the more efficient alternative comparative datamining algorithm for the solution of the Differential Gene Expression (DGE) problem.

\subsubsection{Differential Gene Expression (DGE) Algorithm Limitations}

The Differential Gene Expression (DGE) algorithm demonstrated four computational limitations: 1. terminal quantile computation, 2. terminal expression level measurement computation, 3. local neighborhood computation and 4. time complexity analysis. The terminal quantiles could not be accurately computed using the nonparametric quantile regression algorithm which required the conditions of the dense datapoint densities ( $\mathrm{Yu}$ and Jones 1998). The nonparametric quantile regression algorithm requires extension which would enable the computation of the terminal quantiles under the conditions of the sparse datapoint densities (Yu and Jones 1998). The

terminal expression level measurements could not be accurately computed using the nonparametric quantile regression algorithm which required the conditions of the dense datapoint densities ( $\mathrm{Yu}$ and Jones 1998). The nonparametric quantile regression algorithm requires extension which would enable the computation of the terminal expression level measurements under the conditions of the sparse datapoint densities ( $\mathrm{Yu}$ and Jones 1998). The local neighborhood algorithm has not been subjected to rigorous optimization for the determination of the optimal local neighborhood sizes ( $\mathrm{Yu}$ and Jones 1998). The local neighborhood algorithm requires extension which would enable the computation of the optimal local neighborhood sizes ( $Y u$ and Jones 1998). The time complexity analysis revealed that the quantile computations were the slowest ratelimiting step of the DGE algorithm (Yu and Jones 1998). The quantile computations require the parallel computer implementation. 


\subsubsection{Differential Gene Expression (DGE) Algorithm Significance}

The proof-of-concept experiments provided convincing evidence that the Differential Gene Expression (DGE) algorithm is the more efficient alternative comparative datamining algorithm for the solution of the Differential Gene Expression (DGE) problem. Because of its uses in other applications, we can foresee that the DGE algorithm will advance six fields of contemporary scientific research: 1. molecular target identification in rational drug design (Jayapal and Melendez 2006), 2. identification of genes critical in stem cell differentiation or developmental processes (Aiba et al. 2006), 3. description of genes mediating cellular responses to toxic chemical or radiation physical stimuli (Gatzidou et al. 2007; National Research Council 2007; Ogawa et al. 2007), 4. reverse engineering of pathological mechanisms arising from complex genetic diseases (Nanni et al. 2006), 5. definition of diagnostic biomarkers for complex genetic diseases and pharmaceutical research (He 2006) and 6. classification of pharmacogenomic events associated with pharmaceutical treatments (Villeneuve and Parissenti 2004).

\subsection{Differential Gene Expression (DGE) Algorithm Future Research Directions}

The Differential Gene Expression (DGE) algorithm demonstrated five outstanding research requirements: 1 . statistical gene expression measurement properties, 2 . extended nonparametric quantile regression algorithm, 3. extended local neighborhood algorithm, 4. parallel computer implementation and 5. receiver operating characteristic curves. The DGE algorithm demonstrated three statistical gene expression measurement properties: 1 . nonsymmetric crossover effect, 2. nonsymmetric differential effect and 3. differential fallback effect. The theoretical basis for the statistical gene expression measurement properties remains unknown. The DGE algorithm would be enhanced through the incorporation of the extended nonparametric quantile regression algorithm capable of the computation of the terminal quantiles and the terminal expression level measurements under the conditions of the sparse datapoint densities ( $\mathrm{Yu}$ and Jones 1998). The DGE 
algorithm would be enhanced through the incorporation of the extended local neighborhood algorithm capable of the computation of the optimal local neighborhood sizes. The DGE algorithm would be enhanced through the parallel computer implementation capable of the rapid quantile computations. The DGE algorithm accuracy performance analysis could be further extended by determining the receiver operating characteristic (ROC) curves. The five outstanding research requirements need further investigation. The five outstanding research requirements are posed as open research problems. 


\section{Glossary}

Accuracy: the accuracy statistic was defined as the degree of conformity of the computations or estimates to the exact or true values. The accuracy statistic was the closeness of an observation to the quantity intended to be observed.

Accuracy (AC): the accuracy (AC) statistic was the proportion of total values that were correctly classified. The formula for the AC statistic was written as:

$\mathrm{AC}=((\mathrm{TP}+\mathrm{TN}) /(\mathrm{TP}+\mathrm{FP}+\mathrm{TN}+\mathrm{FN}))$.

$\mathrm{AC}=$ accuracy

$\mathrm{TP}=$ true positive

$\mathrm{FP}=$ false positive

$\mathrm{TN}=$ true negative

$\mathrm{FN}=$ false negative

Accuracy performance analysis: refer to the comparative accuracy performance analysis.

Arbitrary fold-change limits: with arbitrary fold-change limits, the linear fold-change boundaries were not based on rigorous biological criteria and were chosen arbitrarily. Most published research used a postnormalization gene expression ratio of two-fold (increase or decrease) in the transcription measurements to define differential gene expression. There was no rigorous theoretical basis for selecting this linear fold-change threshold as significant. The Linear Fold-Change (LFC) method considered genes with large changes in differential expression corresponding to at least a two-fold increase or a two-fold decrease in the transcription measurements to be significant. The arbitrary choice of nonunit gene expression ratios for identifying differential gene expression introduced divergence between the upper linear fold-change threshold, the microarray dataset and the lower linear fold-change threshold. 
Binary classification system: the binary classification algorithm categorized the data into two predicted classes.

Comparative accuracy performance analysis: the comparative accuracy performance analysis was based on the use of the accuracy statistic. The accuracy statistic was defined as the degree of conformity of the computations or estimates to the exact or true values. The accuracy statistic was the closeness of an observation to the quantity intended to be observed. The comparative accuracy performance analysis was based on the analysis of microarray datasets in which the numbers and the identities of the differentiallyexpressed genes were known in advance. Microarray datasets containing known differentially-expressed genes do not exist. Therefore, it was necessary to use the synthetic microarray datasets method to generate the synthetic microarray datasets containing known differentially-expressed genes.

Comparative microarray analysis: the comparative microarray analysis involved comparisons of the corresponding gene transcription measurements between the control microarray datasets and the experimental microarray datasets in the differential gene expression algorithms.

Complementary sequence hybridization: complementary sequence hybridization referred to the fact that only deoxyribonucleic acid (DNA) single-stranded molecules possessing complementary sequences were capable of mutual hybridization for the formation of stable DNA double-stranded molecules.

Confusion matrix: the confusion matrix contained information about the actual classifications and the predicted classifications performed by the classification system (Kohavi and Provost 1998). 
Differential fallback effect: the differential fallback effect involved the differentials. The intersection point between the quantile graph and the expected quantile graph corresponded to the minimum absolute differential ordered pair $\left(\mathrm{q}_{\mathrm{ma}}, \mathrm{E}\left(\mathrm{q}_{\mathrm{ma}}\right)\right)$.

Differential Gene Expression (DGE) algorithm: the DGE algorithm was developed as the comparative datamining algorithm for the solution of the Differential Gene Expression (DGE) problem. The DGE algorithm was based on the use of the quantile. The quantiles were used for the statistical gene expression measurements in the DGE algorithm.

Differential Gene Expression (DGE) problem: the identification of the genes exhibiting differential expression under the alternate experimental conditions. The DGE problem involved the categorization of the differences between the control state gene transcription measurements and the experimental state gene transcription measurements. The categorization of the differences between the control state gene transcription measurements and the experimental state gene transcription measurements resulted in three possible gene transcription classifications: 1. positive regulation, 2. neutral regulation and 3. negative regulation.

Downregulated genes: refer to negative regulation.

Downregulated / upregulated quantile ratio (DUQR): the downregulated / upregulated quantile ratio (DUQR) was the global relative quantitative measurement of the downregulated quantiles. The DUQR was defined as DUQR $=d_{m p} / d_{m n}$.

$\mathrm{DUQR}=$ downregulated $/$ upregulated quantile ratio

$\mathrm{d}_{\mathrm{mp}}=$ maximum positive differential

$\mathrm{d}_{\mathrm{mn}}=$ maximum negative differential

False discovery rate (FDR): the false discovery rate (FDR) was the percentage of differentials that were incorrectly identified by random chance as differentially-expressed 
gene transcription measurements using the specified differential criterion. The FDR was estimated by the identification of nonsense genes in the $C(6,3)=20$ combinations of the six microarray datasets consisting of the three control microarray datasets and the three experimental microarray datasets.

False negative (FN): the false negative (FN) statistic was the number of predicted negative values which were in reality positive values. The FN statistic was equivalent to the number of missed values. The FN statistic was equivalent to the number of Type II errors.

False negative rate (FNR): the false negative rate (FNR) statistic was the proportion of actual positive values that were incorrectly classified as predicted negative values. The FNR statistic was equivalent to the Type II error rate ( $\beta$ ) statistic. The FNR statistic was equal to 1 minus the sensitivity (SE) statistic. The formula for the FNR statistic was written as:

$\mathrm{FNR}=(\mathrm{FN} /(\mathrm{TP}+\mathrm{FN}))=\beta=(1-\mathrm{SE})$

$\mathrm{FNR}=$ false negative rate

$\mathrm{FN}=$ false negative

$\mathrm{TP}=$ true positive

$\beta=$ Type II error rate

$\mathrm{SE}=$ sensitivity

False positive (FP): the false positive (FP) statistic was the number of predicted positive values which were in reality negative values. The FP statistic was equivalent to the number of false alarm values. The FP statistic was equivalent to the number of Type I errors.

False positive rate (FPR): the false positive rate (FPR) statistic was the proportion of actual negative values that were incorrectly classified as predicted positive values. The 
FPR statistic was equivalent to the Type I error rate $(\alpha)$ statistic. The FPR statistic was equal to 1 minus the specificity (SP) statistic. The formula for the FPR statistic was written as:

$\mathrm{FPR}=(\mathrm{FP} /(\mathrm{TN}+\mathrm{FP}))=\alpha=(1-\mathrm{SP})$

FPR $=$ false positive rate

$\mathrm{FP}=$ false positive

$\mathrm{TN}=$ true negative

$\alpha=$ Type I error rate

$\mathrm{SP}=$ specificity

Fold change: the fold change was the gene expression ratio of microarray transcriptional measurements defined by the formula:

fold change $=($ experimental value $/$ control value $)$

fold change $=$ gene expression ratio experimental value $=$ experimental state microarray transcriptional measurement control value $=$ control state microarray transcriptional measurement

F-measure (FM): the F-measure (FM) statistic was the weighted harmonic mean of the TPR statistic and the PPV statistic. The FM statistic assigned a weight $\beta$ to control the relative contributions of the TPR statistic and the PPV statistic in computing the weighted harmonic mean. The weight $\beta$ can adopt a value from 0 to infinity. The formula for the FM statistic given the weight $\beta$ was written as:

$$
\begin{aligned}
& \mathrm{FM}=\left(\left(\left(\beta^{2}+1\right) * \mathrm{PPV} * \mathrm{TPR}\right) /\left(\beta^{2} * \mathrm{PPV}+\mathrm{TPR}\right)\right) \\
& \mathrm{FM}=\mathrm{F} \text {-measure } \\
& \beta=\text { weight }
\end{aligned}
$$


$\mathrm{PPV}=$ positive predictive value

$\mathrm{TPR}=$ true positive rate

F $_{1}$-measure (F1M): the $F_{1}$-measure (F1M) statistic was the harmonic mean of the TPR statistic and the PPV statistic. The F1M statistic assigned a weight $\beta=1$ to designate the equal contributions of the TPR statistic and the PPV statistic in computing the harmonic mean. The formula for the F1M statistic given the weight $\beta=1$ was written as:

$\mathrm{F} 1 \mathrm{M}=((2 * \mathrm{PPV} * \mathrm{TPR}) /(\mathrm{PPV}+\mathrm{TPR}))$

$\mathrm{F} 1 \mathrm{M}=\mathrm{F}_{1}$-measure

$P P V=$ positive predictive value

$\mathrm{TPR}=$ true positive rate

The F1M statistic represented a restricted case of the FM statistic. The F1M statistic was equivalent to the traditional FM statistic. The F1M statistic was equivalent to the balanced FM statistic (Lewis and Gale 1994).

Geometric-mean $_{1}$ (GM1): the geometric-mean $(\mathrm{GM} 1$ ) statistic was the geometric mean of the true positive rate (TPR) statistic and the positive predictive value (PPV) statistic (Kubat et al. 1998). The formula for the GM1 statistic was written as:

$\mathrm{GM} 1=(\mathrm{TPR} * \mathrm{PPV})^{0.5}$

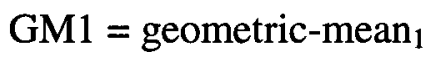

$\mathrm{TPR}=$ true positive rate

$\mathrm{PPV}=$ positive predictive value

Geometric-mean $_{2}$ (GM2): the geometric-mean 2 (GM2) statistic was the geometric mean of the true positive rate (TPR) statistic and the true negative rate (TNR) statistic (Kubat et al. 1998). The formula for the GM2 statistic was written as: 
$\mathrm{GM} 2=(\mathrm{TPR} * \mathrm{TNR})^{0.5}$

$\mathrm{GM} 2=$ geometric - mean $_{2}$

$\mathrm{TPR}=$ true positive rate

$\mathrm{TNR}=$ true negative rate

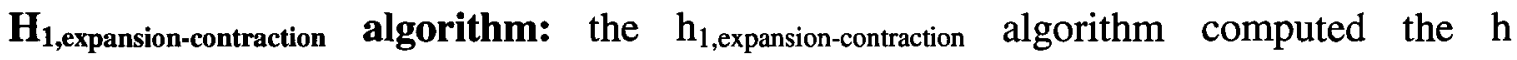
bandwidth which generated the minimum residual squares criterion (RSC) error estimate in the RSC versus bandwidth curve.

$\mathbf{H}_{1 \text {,mean }}$ algorithm: the $h_{1}$ bandwidth was the scale parameter that controlled the amount of $\mathrm{x}$ direction smoothing applied to the regression data. The $\mathrm{h}_{1, \text { mean }}$ algorithm computed the $h_{1, \text { mean }}$ bandwidth which was the optimal choice of the $h_{1}$ bandwidth for the regression mean estimation.

$\mathbf{H}_{1, \text { minimum present }}$ algorithm: the $h_{1, \text { minimum present }}$ algorithm determined whether the minimum residual squares criterion (RSC) error estimate existed in the RSC versus bandwidth curve. The presence of the minimum RSC error estimate indicated that the RSC versus bandwidth curve was nonmonotonic. The absence of the minimum RSC error estimate indicated that the RSC versus bandwidth curve was monotonic.

$\mathbf{H}_{1, p}$ algorithm: the $h_{1}$ bandwidth was the scale parameter that controlled the amount of $x$ direction smoothing applied to the regression data. The $\mathrm{h}_{1, \mathrm{p}}$ bandwidth was the scale parameter that controlled the amount of $\mathrm{x}$ direction smoothing applied to the regression data for the $\mathrm{p}$ percentile.

$\mathbf{H}_{2, p}$ algorithm: the $h_{2}$ bandwidth was the scale parameter that controlled the amount of $y$ direction smoothing applied to the regression data. The $h_{2, p}$ bandwidth was the scale parameter that controlled the amount of y direction smoothing applied to the regression data for the $p$ percentile. 
Implicit estimation function: the implicit estimation function computed the successive approximation of the nonparametric quantile regression. The implicit estimation function was defined:

$\mathrm{q}_{\text {new }, \mathrm{p}}\left(\mathrm{x}_{\text {grid }}\right)=\left(\left(\mathrm{m}\left(\mathrm{x}_{\text {grid }}\right)\right)+\left(((2 * \mathrm{p})-1) * \mathrm{~h}_{2, \mathrm{p}}\right)+\left(\left(\left(\Sigma_{\mathrm{j}}\left(\left(\mathrm{q}_{\text {old }, \mathrm{p}}\left(\mathrm{x}_{\text {grid }}\right)\right)-\mathrm{y}_{\text {data, }}-\mathrm{h}_{2, \mathrm{p}}\right)\right) *\left(\mathrm{w}_{\mathrm{j}}\right.\right.\right.\right.$

$\left.\left.\left.\left(\mathrm{x}_{\text {grid }}\right)\right) *\left(\mathrm{I}\left(\mathrm{y}_{\text {data, }} \leq\left(\left(\mathrm{q}_{\text {old }, \mathrm{p}}\left(\mathrm{x}_{\text {grid }}\right)\right)-\mathrm{h}_{2, \mathrm{p}}\right)\right)\right)\right) /\left(\Sigma_{\mathrm{j}}\left(\mathrm{w}_{\mathrm{j}}\left(\mathrm{x}_{\text {grid }}\right)\right)\right)\right)+\left(\left(\left(\Sigma_{\mathrm{j}}\left(\left(\mathrm{q}_{\text {old }, \mathrm{p}}\left(\mathrm{x}_{\text {grid }}\right)\right)-\mathrm{y}_{\text {data }, \mathrm{j}}+\right.\right.\right.\right.$ $\left.\left.\left.\left.\left.\mathrm{h}_{2, \mathrm{p}}\right)\right) *\left(\mathrm{w}_{\mathrm{j}}\left(\mathrm{x}_{\text {grid }}\right)\right) *\left(\mathrm{I}\left(\mathrm{y}_{\text {data } \mathrm{j}} \geq\left(\left(\mathrm{q}_{\text {old }, \mathrm{p}}\left(\mathrm{x}_{\text {grid }}\right)\right)+\mathrm{h}_{2, \mathrm{p}}\right)\right)\right)\right) /\left(\Sigma_{\mathrm{j}}\left(\mathrm{w}_{\mathrm{j}}\left(\mathrm{x}_{\text {grid }}\right)\right)\right)\right)\right)$

$\mathrm{h}_{2, \mathrm{p}}=$ bandwidth which was the scale parameter that controlled the amount of $\mathrm{y}$ direction smoothing applied to the regression data for the $p$ percentile.

$\mathrm{I}=$ identity function.

$\mathrm{m}\left(\mathrm{x}_{\mathrm{grid}}\right)=$ local linear kernel estimate at the $\mathrm{x}_{\text {grid }}$ point.

$\mathrm{p}=$ percentile.

$\mathrm{q}_{\text {new, }}\left(\mathrm{x}_{\text {grid }}\right)=$ next estimate of the $\mathrm{p}$ percentile at the $\mathrm{x}_{\text {grid }}$ point.

$\mathrm{q}_{\text {old }, \mathrm{p}}\left(\mathrm{x}_{\text {grid }}\right)=$ previous estimate of the $\mathrm{p}$ percentile at the $\mathrm{x}_{\text {grid }}$ point.

$\mathrm{w}_{\mathbf{j}}\left(\mathrm{x}_{\text {grid }}\right)=$ weight between the $\mathrm{x}_{\text {grid }}$ point and the $\mathrm{x}_{\text {dataj }}$ point.

$\mathrm{x}_{\mathrm{data}, \mathrm{j}}=\mathrm{x}$ datapoint $\mathrm{j}$.

$\mathrm{x}_{\text {grid }}=\mathrm{x}$ gridpoint.

$y_{\text {data, }}=y$ datapoint $j$.

Identity (I) function: the identity (I) function evaluated a Boolean logic function.

Kernel localization function: the kernel localization function $\mathrm{K}$ was the symmetric probability density function. The kernel localization function $\mathrm{K}$ was the local weighting function that assigned high weights to the values which were close to the argument $\mathrm{z}$. The kernel localization function $\mathrm{K}$ was defined:

$\mathrm{K}(\mathrm{z} / \mathrm{h})=(1 /(\sqrt{ }(2 * \pi))) *\left(\operatorname{exponent}\left(\left(-\left(\mathrm{z}^{2}\right)\right) /\left(2 * \mathrm{~h}^{2}\right)\right)\right)$ 
$\mathrm{h}=$ bandwidth which was the scale parameter that controlled the amount of smoothing applied to the regression data.

$\mathrm{K}=$ symmetric probability density function value.

$\mathrm{z}=$ input variable.

Kernel weighted local linear fitting algorithm: the Differential Gene Expression (DGE) algorithm was based on the Nonlinear Fold-Change (NFC) method. The NFC method was based on the nonparametric quantile regression analysis. The nonparametric quantile regression analysis was implemented using the kernel weighted local linear fitting algorithm to approximate conditional quantile functions ( $\mathrm{Yu}$ and Jones 1998). The nonparametric quantile regression analysis was used for the estimation of the gene ranks. The generation of the more stable gene rank estimates led to the more effective identification of differential gene expression.

Linear Fold-Change (LFC) method: the LFC method used two parallel linear foldchange boundaries which were chosen as the criteria to identify the set of genes exhibiting the most statistically-significant variation between the control samples and the experimental samples.

Local linear kernel estimation function: the local linear kernel estimation function $\mathrm{m}$ was the nonparametric estimate of the conditional mean observation. The local linear kernel estimation function $\mathrm{m}$ was defined:

$\mathrm{m}=\left(\left(\Sigma_{\mathrm{j}} \mathrm{w}_{\mathrm{j}}\left(\mathrm{x}_{\text {grid }}\right)\right)^{-1} *\left(\Sigma_{\mathrm{j}} \mathrm{w}_{\mathrm{j}}\left(\mathrm{x}_{\text {grid }}\right) * \mathrm{y}_{\text {data }, \mathrm{j}}\right)\right)$

$w_{j}=$ weight $j$.

$\mathrm{x}_{\text {grid }}=\mathrm{x}$ gridpoint.

$\mathrm{y}_{\mathrm{data}, \mathrm{j}}=\mathrm{y}$ datapoint $\mathrm{j}$.

$\mathrm{m}=$ local linear kernel estimation function value. 
Local neighborhood algorithm: the local neighborhhood algorithm computed the immediate vicinities of the quantiles. The immediate vicinities of the quantiles identified the local datapoints which were used as the input for the computation of the quantiles. The local neighborhood algorithm has not been subjected to rigorous optimization for the determination of the optimal local neighborhood sizes (Yu and Jones 1998). The local neighborhood algorithm requires extension which would enable the computation of the optimal local neighborhood sizes (Yu and Jones 1998).

Local neighborhoods: the local neighborhoods defined the immediate vicinities of the quantiles. The immediate vicinities of the quantiles identified the local datapoints which were used as the input for the computation of the quantiles.

\section{Locally-weighted regression and smoothing scatterplots (LOESS; LOWESS): a} robust estimation method known as the locally-weighted regression and smoothing scatterplots (LOESS or LOWESS) algorithm was used to guard against deviant point distortion of the smoothed points caused by the bias of unsymmetric data distributions. The LOWESS algorithm prevented deviant point distortion of the smoothed points using an adaptation of the iteratively reweighted least squares algorithm.

Lower boundary (LB): the lower boundary (LB) was the lower class boundary in the frequency distribution.

Lower linear fold-change threshold: differential gene expression algorithms were based on the strategy which we referred to as the Linear Fold-Change (LFC) method. The LFC method used two parallel linear fold-change boundaries which were chosen as the criteria to identify the set of genes exhibiting the most statistically-significant variation between the control samples and the experimental samples.

Lower nonlinear fold-change threshold: the DGE algorithm was based on the alternate strategy which we referred to as the Nonlinear Fold-Change (NFC) method. The NFC method used two nonparallel nonlinear fold-change boundaries which were chosen as the 
criteria to identify the set of genes exhibiting the most statistically-significant variation between the control samples and the experimental samples.

Matrix inversion algorithm: the matrix inversion algorithm computed the inverse $\mathrm{S}^{-1}$ of the $2 \times 2$ square matrix $S$.

Maximum negative differential: the maximum positive differential $d_{m p}=E\left(q_{m p}\right)-q_{m p}$ corresponded to the ordered pair $\left(\mathrm{q}_{\mathrm{mp}}, \mathrm{E}\left(\mathrm{q}_{\mathrm{mp}}\right)\right)$. The maximum negative differential $\mathrm{d}_{\mathrm{mn}}=$ $E\left(q_{m n}\right)-q_{m n}$ corresponded to the ordered pair $\left(q_{m n}, E\left(q_{m n}\right)\right)$. The maximum positive differential $d_{m p}$ was used indirectly for the selection of the maximum positive differential quantile $\mathrm{q}_{\mathrm{mp}}$. The maximum negative differential $d_{m n}$ was used indirectly for the selection of the maximum negative differential quantile $\mathrm{q}_{\mathrm{mn}}$.

Maximum positive differential: the maximum positive differential $d_{m p}=E\left(q_{m p}\right)-q_{m p}$ corresponded to the ordered pair $\left(q_{m p}, E\left(q_{m p}\right)\right)$. The maximum negative differential $d_{m n}=$ E $\left(q_{m n}\right)-q_{m n}$ corresponded to the ordered pair $\left(q_{m n}, E\left(q_{m n}\right)\right)$. The maximum positive differential $d_{m p}$ was used indirectly for the selection of the maximum positive differential quantile $\mathrm{q}_{\mathrm{mp}}$. The maximum negative differential $d_{\mathrm{mn}}$ was used indirectly for the selection of the maximum negative differential quantile $\mathrm{q}_{\mathrm{mn}}$.

Microarray technologies: microarray technologies were high-throughput analytical devices that provided comprehensive genome-scale expression analysis by the simultaneous monitoring of quantitative transcription measurements of thousands of genes in parallel.

Molecular Target Identification (MTI) problem: the Molecular Target Identification (MTI) problem referred to the discovery of the molecular targets amenable to pharmaceutical intervention in order to prevent, stabilize or reverse diseases. 
Negative predictive value (NPV): the negative predictive value (NPV) statistic was the proportion of predicted negative values that were correctly classified as actual negative values. The formula for the NPV statistic was written as:

$\mathrm{NPV}=(\mathrm{TN} /(\mathrm{TN}+\mathrm{FN}))$

$\mathrm{NPV}=$ negative predictive value

$\mathrm{TN}=$ true negative

$\mathrm{FN}=$ false negative

Negative regulation: the negative regulation case signified that the control state gene transcription measurements were larger than the experimental state gene transcription measurements. The negative regulation classification was indicative of repressed gene transcription.

Neutral regulation: the neutral regulation case signified that the control state gene transcription measurements were equal to the experimental state gene transcription measurements. The neutral regulation classification was indicative of unchanged gene transcription.

Nonlinear Fold-Change (NFC) method: the NFC method used two nonparallel nonlinear fold-change boundaries which were chosen as the criteria to identify the set of genes exhibiting the most statistically-significant variation between the control samples and the experimental samples.

Nonlinear function: the nonparametric quantile regression did not require the assumption of the linear function between the gene expression levels and gene variance changes. 
Nonparametric quantile regression algorithm: the nonparametric quantile regression algorithm was implemented using the kernel weighted local linear fitting algorithm to approximate conditional quantile functions ( $\mathrm{Yu}$ and Jones 1998).

Nonregulated genes: refer to neutral regulation.

Nonsymmetric crossover effect: the nonsymmetric crossover effect involved the intersection point between the two graphs. The intersection point between the two graphs corresponded to the minimum absolute differential ordered pair $\left(s_{\operatorname{ma}}, E\left(s_{m a}\right)\right)$. The minimum absolute differential difference statistic $s_{\mathrm{ma}}$ was not equal to the midpoint difference statistic $s_{(n / 2)}, s_{m a} \neq s_{(n / 2)}$. The intersection point between the two graphs exhibited the nonsymmetric crossover effect.

Nonsymmetric differential effect: the nonsymmetric differential effect involved the initial differential $d_{1}$ and the final differential $d_{n}$. The initial differential $d_{1}$ was not equal in size to the final differential $d_{n}, d_{1} \neq d_{n}$. The initial differential $d_{1}$ and the final differential $d_{n}$ exhibited the nonsymmetric differential effect.

Normalization algorithms: normalization algorithms for comparative microarray analysis eliminated the systematic biases between the raw microarray datasets by the systematic error adjustments to the individual hybridization intensities.

Positive predictive value (PPV): the positive predictive value (PPV) statistic was the proportion of predicted positive values that were correctly classified as actual positive values. The PPV statistic was equivalent to the precision (PR) statistic. The formula for the PPV statistic was written as:

$\mathrm{PPV}=(\mathrm{TP} /(\mathrm{TP}+\mathrm{FP}))=\mathrm{PR}$.

$\mathrm{PPV}=$ positive predictive value

$\mathrm{TP}=$ true positive 
$\mathrm{FP}=$ false positive

$\mathrm{PR}=$ precision

Positive regulation: the positive regulation case signified that the control state gene transcription measurements were smaller than the experimental state gene transcription measurements. The positive regulation classification was indicative of induced gene transcription.

Power (P): refer to the true positive rate (TPR).

Precision: the precision statistic was distinguished from the accuracy statistic. The precision statistic was defined as the spread of estimates of a parameter in a statistical model. The precision statistic was the quality associated with the class of measurements and referred to the way in which repeated observations conformed to themselves. The precision statistic referred to the dispersion of the observations whether or not the mean value around which the dispersion was measured approximated the true value.

Precision (PR): refer to the positive predictive value (PPV).

Quantiles: the quantiles were points taken at regular intervals from the cumulative distribution function of a random variable. Dividing ordered data into $q$ essentially equal-sized subsets was the motivation for the q-quantiles. The quantiles were the data values that marked the boundaries between the consecutive subsets. The kth q-quantile was the value $\mathrm{x}$ such that the probability that a random variable will be less than $\mathrm{x}$ is at most $\mathrm{k} / \mathrm{q}$ and the probability that a random variable will be more than or equal to $\mathrm{x}$ is at least $\mathrm{k} / \mathrm{q}$.

Quantile initialization algorithm: the quantile initialization algorithm computed the initial values of the quantiles. 
Quantile tolerance function: the quantile tolerance function computed the relative difference between the next estimate of the y grid point and the previous estimate of the $y$ grid point. The quantile tolerance function was defined:

tolerance $=\left(\left(\right.\right.$ absolute $\left.\left(\mathrm{y}_{\text {grid,new }}-\mathrm{y}_{\text {grid,old }}\right)\right) /\left(\right.$ absolute $\left.\left.\left(\mathrm{y}_{\text {grid,old }}\right)\right)\right)$

tolerance $=$ relative difference between the next estimate of the y gridpoint and the previous estimate of the $y$ gridpoint.

$\mathrm{y}_{\text {grid,old }}=$ previous estimate of the $\mathrm{y}$ gridpoint.

$\mathrm{y}_{\text {grid,new }}=$ next estimate of the $\mathrm{y}$ gridpoint.

Recall (RE): refer to the true positive rate (TPR).

Receiver operating characteristic (ROC) curve: in signal detection theory, the receiver operating characteristic (ROC) curve was the graph of the sensitivity versus (1 specificity) for the binary classification system during the systematic variation of the discrimination threshold. The ROC curve was also represented by plotting the true positive rate (TPR) versus the false positive rate (FPR). The ROC curve was also referred to as the Relative Operating Characteristic curve because during the systematic change of the discrimination criterion, it was the comparison of the two operating characteristics: 1 . TPR and 2. FPR.

Regression analysis: regression analysis was defined as a mathematical technique that formulated a mathematical model of the relationship between a set of $q$ independent variables $x_{1}, x_{2}, x_{3} \ldots x_{q}$ and a dependent variable $y$. The choice of the explicit form of the model was based on such considerations as the smoothness and continuity of the dependent variable $\mathrm{y}$ as a function of the $\mathrm{q}$ independent variables $\mathrm{x}_{1}, \mathrm{x}_{2}, \mathrm{x}_{3} \ldots \mathrm{x}_{\mathrm{q}}$. Regression models were defined to be of the general form:

$$
y=f\left(x_{1}, x_{2}, x_{3} \ldots x_{q}\right)+\varepsilon
$$


The function $\mathrm{f}$ specified the relationship between the $\mathrm{q}$ independent variables $\mathrm{x}_{1}, \mathrm{x}_{2}, \mathrm{x}_{3} \ldots$ $\mathrm{x}_{\mathrm{q}}$ and the dependent variable $\mathrm{y}$. The random error $\varepsilon$ indicated the reliance of the dependent variable $y$ on quantities other than the $q$ independent variables $x_{1}, x_{2}, x_{3} \ldots x_{q}$.

Regulated genes: the regulated genes were a composite statistical gene expression measurement category which included both the downregulated genes and the upregulated genes.

Residual squares criterion (RSC) algorithm: the residual squares criterion (RSC) algorithm computed the error estimate of the $h$ bandwidth.

Residual squares criterion (RSC) weight function: the residual squares criterion (RSC) weight function computed the RSC weight between two datapoints. The RSC weight function was defined:

weight $=\left(\left(\mathrm{e}^{* *}\left(\left(-1 *\left(\left(\mathrm{x}_{\mathrm{i}}-\mathrm{x}_{\mathrm{j}}\right)^{2}\right)\right) /\left(2 *\left(\mathrm{~h}_{1}\right)^{2}\right)\right)\right) /(\sqrt{ }(2 * \pi))\right)$

$\mathrm{h}_{1}=$ bandwidth which was the scale parameter that controlled the amount of $\mathrm{x}$ direction smoothing applied to the regression data.

weight $=$ residual squares criterion weight between the $x$ datapoint $i$ and the $x$ datapoint $j$. $\mathrm{x}_{\mathrm{i}}=\mathrm{x}$ datapoint $\mathrm{i}$.

$\mathrm{x}_{\mathrm{j}}=\mathrm{x}$ datapoint $\mathrm{j}$.

Sensitivity (SE): refer to the true positive rate (TPR).

Signal variance: signal variance referred to the statistical variation in the gene transcription signal-to-noise ratios detected using the microarray technologies.

Significance Analysis of Microarrays (SAM) algorithm: the SAM algorithm was developed as the comparative datamining algorithm for the solution of the Differential Gene Expression (DGE) problem. The SAM algorithm was based on the use of the 
modified T-statistic. The modified T-statistic was referred to as the difference statistic. The difference statistics were used for the statistical gene expression measurements in the SAM algorithm.

$S_{n, l}$ function: the $S_{n, l}$ function was the weighted sum of the kernel localization functions that was used to define the weight function.

Specificity (SP): refer to the true negative rate (TNR).

Standard normal density function ( $\phi)$ : the normal distribution or Gaussian distribution formed a family of continuous probability distributions. Each member of the family was defined by two parameters: the mean $(\mu)$ and the variance $\left(\sigma^{2}\right)$. The standard normal distribution was the normal distribution with a mean of zero and a variance of one. The probability density function (pdf) for the normal distribution (standard normal density function $(\phi))$ was defined by the formula:

$\phi=\left(\left(1 /\left(\sigma^{*}(\operatorname{sqrt}(2 * \pi))\right)\right) *\left(\exp \left(-\left(\left((\mathrm{x}-\mu)^{2}\right) /\left(2 * \sigma^{2}\right)\right)\right)\right)\right)$

$\phi=$ standard normal density function

$\sigma=$ standard deviation $=1$

$\pi=\mathrm{pi}=3.14159 \ldots$

$\mathrm{x}=$ argument

$\mu=$ mean $=0$

Standard normal distribution function $(\Phi)$ : the normal distribution or Gaussian distribution formed a family of continuous probability distributions. Each member of the family was defined by two parameters: the mean $(\mu)$ and the variance $\left(\sigma^{2}\right)$. The standard normal distribution was the normal distribution with a mean of zero and a variance of one. The cumulative distribution function (cdf) for the normal distribution (standard normal distribution function $(\Phi)$ ) was defined by the formula: 
$\Phi=((1 / 2) *(1+(\operatorname{erf}((\mathrm{x}-\mu) /(\sigma *(\operatorname{sqrt} 2))))))$

$\Phi=$ standard normal distribution function

erf $=$ error function

$\mathrm{x}=$ argument

$\mu=$ mean $=0$

$\sigma=$ standard deviation $=1$

Statistical gene expression measurement categories: the statistical gene expression measurement categories involved the classification of the statistical gene expression measurements into the different categories. The Significance Analysis of Microarrays (SAM) algorithm classified the statistical gene expression measurements into two categories: 1. nonregulated genes and 2. regulated genes. The SAM algorithm statistical gene expression measurement categories did not differentiate between the downregulated genes and the upregulated genes. The Differential Gene Expression (DGE) algorithm classified the statistical gene expression measurements into three categories: 1. downregulated genes, 2. nonregulated genes and 3. upregulated genes. The DGE algorithm statistical gene expression measurement categories differentiated between the downregulated genes and the upregulated genes. The DGE algorithm offered an increased number of more detailed statistical gene expression measurement categories.

Statistical gene expression measurement classification error fault tolerance: the statistical gene expression measurement classification error fault tolerance involved the fault tolerance to the statistical gene expression measurement classification errors caused by two statistical gene expression measurement properties: 1 . nonsymmetric crossover effect and 2. nonsymmetric differential effect.

Synthetic downregulated genes method: the synthetic downregulated genes method transformed the natural microarray datasets. The randomly-chosen genes subset was 
subjected to the negative y value alteration. The negative y value alteration produced the downregulated genes subset.

Synthetic microarray datasets method: the synthetic microarray datasets method was used to generate the synthetic microarray datasets containing known differentiallyexpressed genes. The availability of the synthetic microarray datasets in which the numbers and the identities of the differentially-expressed genes were known in advance formed the basis for the comparative accuracy performance analysis. The synthetic microarray datasets method consisted of the two component methods: 1. synthetic upregulated genes method and 2. synthetic downregulated genes method. The synthetic upregulated genes method transformed the natural microarray datasets.

Synthetic upregulated genes method: the synthetic upregulated genes method transformed the natural microarray datasets. The randomly-chosen genes subset was subjected to the positive y value alteration. The positive y value alteration produced the upregulated genes subset.

Terminal expression level measurement computation: refer to the terminal expression level measurements.

Terminal quantiles: the terminal quantiles consisted of two sets of quantiles: 1. initial quantiles and 2. final quantiles. The initial quantiles included the quantiles which were lesser than the 0.01 quantile, $\mathrm{q}_{\text {initial }}<0.01$. The final quantiles included the quantiles which were greater than the 0.99 quantile, $0.99<\mathrm{q}_{\text {final. }}$.

Time complexity analysis: the time complexity analysis of an algorithm was the amount of time required by the algorithm expressed as a function of the size of the problem. The asymptotic time complexity was the limiting factor of the complexity as the problem size increased. The asymptotic complexity of an algorithm determined the maximum size of the problems that could be solved by the algorithm. 
T-statistic: the T-statistic was a statistic whose sampling distribution was the $\mathrm{T}$ distribution. The T-statistic was a measure of the extremity of a statistical measure. The T-statistic was computed by subtracting the hypothesized value from the statistical estimate and then dividing by the estimated standard error. The hypothesized value was frequently zero.

Total discovery rate (TDR): the total discovery rate (TDR) was the percentage of differentials that were identified as differentially-expressed gene transcription measurements using the specified differential criterion.

Transcriptional variation: transcriptional variation referred to the fact that gene expression was primarily regulated at the transcriptional level. The predominant factor underlying gene expression alteration was quantitative variation in the transcriptional production of messenger ribonucleic acid (mRNA) molecules.

True negative (TN): the true negative (TN) statistic was the number of predicted negative values which were in reality negative values. The TN statistic was equivalent to the number of correctly rejected values.

True negative rate (TNR): the true negative rate (TNR) statistic was the proportion of actual negative values that were correctly classified as predicted negative values. The TNR statistic was equivalent to the specificity (SP) statistic. The formula for the TNR statistic was written as:

$\mathrm{TNR}=(\mathrm{TN} /(\mathrm{TN}+\mathrm{FP}))=\mathrm{SP}$

$\mathrm{TNR}=$ true negative rate

$\mathrm{TN}=$ true negative

$\mathrm{FP}=$ false positive

$\mathrm{SP}=$ specificity 
True positive (TP): the true positive (TP) statistic was the number of predicted positive values which were in reality positive values. The TP statistic was equivalent to the number of hit values.

True positive rate (TPR): the true positive rate (TPR) statistic was the proportion of actual positive values that were correctly classified as predicted positive values. The TPR statistic was equivalent to the sensitivity (SE) statistic. The TPR statistic was equivalent to the recall (RE) statistic. The TPR statistic was equivalent to the power (P) statistic. The formula for the TPR statistic was written as:

$\mathrm{TPR}=(\mathrm{TP} /(\mathrm{TP}+\mathrm{FN}))=\mathrm{SE}=\mathrm{RE}=\mathrm{P}$

$\mathrm{TPR}=$ true positive rate

$\mathrm{TP}=$ true positive

$\mathrm{FN}=$ false negative

$\mathrm{SE}=$ sensitivity

$\mathrm{RE}=$ recall

$\mathrm{P}=$ power

Type I error rate ( $\alpha)$ : refer to the false positive rate (FPR).

Type II error rate ( $\beta$ ): refer to the false negative rate (FNR).

Upper boundary (UB): the upper boundary (UB) was the upper class boundary in the frequency distribution.

Upper linear fold-change threshold: differential gene expression algorithms were based on the strategy which we referred to as the Linear Fold-Change (LFC) method. The LFC method used two parallel linear fold-change boundaries which were chosen as the criteria to identify the set of genes exhibiting the most statistically-significant variation between the control samples and the experimental samples. 
Upper nonlinear fold-change threshold: the DGE algorithm was based on the alternate strategy which we referred to as the Nonlinear Fold-Change (NFC) method. The NFC method used two nonparallel nonlinear fold-change boundaries which were chosen as the criteria to identify the set of genes exhibiting the most statistically-significant variation between the control samples and the experimental samples.

Upregulated genes: refer to positive regulation.

Upregulated / downregulated quantile ratio (UDQR): the upregulated / downregulated quantile ratio (UDQR) was the global relative quantitative measurement of the upregulated quantiles. The UDQR was defined as $U D Q R=d_{m n} / d_{m p}$.

$\mathrm{UDQR}=$ upregulated $/$ downregulated quantile ratio

$\mathrm{d}_{\mathrm{mp}}=$ maximum positive differential

$\mathrm{d}_{\mathrm{mn}}=$ maximum negative differential

Variance identification: the variance identification referred to the qualitative set of genes exhibiting the most statistically-significant variation between the control samples and the experimental samples which was selected for further data analysis.

Weight function: the weight function $w$ was associated with the local linear fitting. The weight function $w$ was used to construct the matrix $S$ and the matrix $\mathrm{S}^{*}$ that formed the core of the residual squares criterion (RSC) algorithm. The weight function w was defined:

$\mathrm{w}_{\mathrm{j}}\left(\mathrm{x}_{\text {grid }}\right)=\left(\left(\mathrm{K}\left(\left(\mathrm{x}_{\text {grid }}-\mathrm{x}_{\text {data, }}\right) / \mathrm{h}_{1}\right)\right) *\left(\mathrm{~S}_{\mathrm{n}, 2}-\left(\left(\mathrm{x}_{\text {grid }}-\mathrm{x}_{\text {data, }}\right) * \mathrm{~S}_{\mathrm{n}, 1}\right)\right)\right)$

$\mathrm{h}_{1}=$ bandwidth which was the scale parameter that controlled the amount of $\mathrm{x}$ direction smoothing applied to the regression data.

$\mathrm{K}=$ kernel localization function. 
$\mathrm{S}_{\mathrm{n}, 1}=\mathrm{S}_{\mathrm{n}, 1}$ function.

$S_{n, 2}=S_{n, 2}$ function.

$\mathrm{w}=$ weight between the $\mathrm{x}$ gridpoint and the $\mathrm{x}$ datapoint $\mathrm{j}$.

$\mathrm{x}_{\text {data, } \mathrm{j}}=\mathrm{x}$ datapoint $\mathrm{j}$.

$\mathrm{x}_{\text {grid }}=\mathrm{x}$ gridpoint . 


\section{References}

Aiba, K., Carter, M.G., Matoba, R. and Ko, M.S. Genomic approaches to early embryogenesis and stem cell biology. Semin. Reprod. Med. 24 (5): 330-339, 2006.

Aittokallio, T., Kurki, M., Nevalainen, O., Nikula, T., West, A. and Lahesmaa, R. Computational strategies for analyzing data in gene expression microarray experiments. J. Bioinf. Comput. Biol. 1 (3): 541-586, 2003.

Ayroles, J.F. and Gibson, G. Analysis of variance of microarray data. Methods Enzymol. 411:214-233, 2006.

Barrett, T., and R. Edgar. Gene Expression Omnibus: microarray data storage, submission, retrieval, and analysis. Methods Enzymol. 411:352-369, 2006a.

Barrett, T., and R. Edgar. Mining microarray data at NCBI's Gene Expression Omnibus (GEO). Methods Mol. Biol. 338:175-190, $2006 \mathrm{~b}$.

Barrett, T., T.O. Suzek, D.B. Troup, S.E. Wilhite, W.C. Ngau, P. Ledoux, D. Rudnev, A.E. Lash, W. Fujibuchi, and R. Edgar. NCBI GEO: mining millions of expression profiles - database and tools. Nucleic Acids Res. 33 (Database): D562-D566, 2005.

Barrett, T., D.B. Troup, S.E. Wilhite, P. Ledoux, D. Rudnev, C. Evangelista, I.F. Kim, A. Soboleva, M. Tomashevsky, and R. Edgar. NCBI GEO: mining tens of millions of expression profiles - database and tools update. Nucleic Acids Res. 35 (Database): D760D765, 2007.

Benes, V. and Muckenthaler, M. Standardization of protocols in cDNA microarray analysis. Trends Biochem. Sci. 28 (5): 244-249, 2003. 
Bilban, M., Buehler, L.K., Head, S., Desoye, G. and Quaranta, V. Normalizing DNA microarray data. Curr. Issues Mol. Biol. 4 (2): 57-64, 2002.

Black, E.P., E. Huang, H. Dressman, R. Rempel, N. Laakso, S.L. Asa, S. Ishida, M. West, and J.R. Nevins. Distinct gene expression phenotypes of cells lacking $\mathrm{Rb}$ and $\mathrm{Rb}$ family members. Cancer Res. 63 (13): 3716-3723, 2003.

Bobashev, G.V., Das, S. and Das, A. Experimental design for gene microarray experiments and differential expression analysis. In Methods of microarray data analysis II: papers from CAMDA '01, ed. S.M. Lin and K.F. Johnson, 23-41. Norwell, MA: Kluwer Academic Publishers, 2002.

Carulli, J.P., Artinger, M., Swain, P.M., Root, C.D., Chee, L., Tulig, C., Guerin, J., Osborne, M., Stein, G., Lian, J. and Lomedico, P.T. High throughput analysis of differential gene expression. J. Cell. Biochem. Suppl. 30/31:286-296, 1998.

Chen, C. and Wei, Y. Computational issues for quantile regression. Sankhya 67 (2): 399$417,2005$.

Chen, Z.J., Gate, L., Davis, W., Ile, K.E. and Tew, K.D. Integration of amplified differential gene expression (ADGE) and DNA microarray. Life 54 (6): 335-338, 2002.

Chen, Y., Yakhini, Z., Ben-Dor, A., Dougherty, E., Trent, J.M. and Bittner, M. Analysis of expression patterns: the scope of the problem, the problem of scope. Dis. Markers 17 (2): 59-65, 2001.

Chu, T.M., Deng, S. and Wolfinger, R.D. Modeling Affymetrix data at the probe level. In DNA microarrays and related genomics techniques: design, analysis and interpretation of experiments, ed. D.B. Allison, G.P. Page, T.M. Beasley and J.W. Edwards, 197-222. Boca Raton, FL: CRC Pr., 2006. 
Churchill, G.A. Using ANOVA to analyze microarray data. BioTechniques 37 (2): 173 177, 2004.

Claverie, J.M. Computational methods for the identification of differential and coordinated gene expression. Hum. Mol. Genet. 8 (10): 1821-1832, 1999.

Cleveland, W.S. Robust locally weighted regression and smoothing scatterplots. J. Amer. Statist. Assoc. 74 (368): 829-836, 1979.

Cleveland, W.S. and Devlin, S.J. Locally weighted regression: an approach to regression analysis by local fitting. J. Amer. Statist. Assoc. 83 (403): 596-610, 1988.

Cleveland, W.S., Devlin, S.J. and Grosse, E. Regression by local fitting: methods, properties and computational algorithms. J. Econometrics 37 (1): 87-114, 1988.

Cleveland, W.S. and Loader, C. Smoothing by local regression: principles and methods. In Statistical theory and computational aspects of smoothing: proceedings of the compstat '94 satellite meeting held in Semmering, Austria, 27-28 august 1994, ed. W. Haerdle and M. Schimek, 10-49. New York, NY: Physica-Verlag, 1996.

Cui, X. and Churchill, G.A. Statistical tests for differential expression in cDNA microarray experiments. Genome Biology 4 (4): 210.1-210.7, 2003.

Do, J.H. and Choi, D.K. Normalization of microarray data: single-labeled and duallabeled arrays. Mol. Cells 22 (3): 254-261, 2006.

Dobbin, K., Shih, J.H. and Simon, R. Questions and answers on design of dual-label microarrays for identifying differentially expressed genes. J. Natl. Cancer Inst. 95 (18): 1362-1369, 2003. 
Draghici, S. Statistical intelligence: effective analysis of high-density microarray data. Drug Discovery Today 7 (11 Suppl.): S55-S63, 2002.

Edgar, R., M. Domrachev, and A.E. Lash. Gene Expression Omnibus: NCBI gene expression and hybridization array data depository. Nucleic Acids Res. 30 (1): 207-210, 2002.

Edwards, J.W. and Ghosh, P. Bayesian analysis of microarray data. In DNA microarrays and related genomics techniques: design, analysis and interpretation of experiments, ed. D.B. Allison, G.P. Page, T.M. Beasley and J.W. Edwards, 267-288. Boca Raton, FL: CRC Pr., 2006.

Elvidge, G. Microarray expression technology: from start to finish. Pharmacogenomics 7 (1): 123-134, 2006.

Fauntleroy, J.C. and Wegman, E.J. Parallelizing locally-weighted regression. Comput. Sci. Statist. 27:327-331, 1996.

Finkelstein, D., Ewing, R., Gollub, J., Sterky, F., Cherry, J.M. and Somerville, S. Microarray data quality analysis: lessons from the AFGC project. Plant Mol. Biol. 48 (12): 119-131, 2002.

Frohme, M., Hauser, N., Scheideler, M., Diehl, F., Beier, M., Wurtz, S. and Hoheisel, J.D. Chips, arrays and other hybridization based techniques for genome and transcriptome characterisation. Biomed. Health Res. 41:88-94, 2000.

Gatzidou, E.T., Zira, A.N. and Theocharis, S.E. Toxicogenomics: a pivotal piece in the puzzle of toxicological research. J. Appl. Toxicol. 27 (4): 302-309, 2007. 
Goldstein, D.R. and Delorenzi, M. Statistical design and data analysis for microarray experiments. In Unraveling lipid metabolism with microarrays, ed. A. Berger and M.A. Roberts, 1-34. New York, NY: Dekker, 2005.

Grosse, E. LOESS: multivariate smoothing by moving least squares. In Approximation theory VI, ed. C.K. Chui, L.L. Schumaker and J.D. Ward, Vol. I, 299-302. San Diego, CA: Academic Pr., 1989.

Gusnanto, A., Calza, S. and Pawitan, Y. Identification of differentially expressed genes and false discovery rate in microarray studies. Curr. Opin. Lipidol. 18 (2): 187-193, 2007.

Hackl, H., Cabo, F.S., Sturn, A., Wolkenhauer, O. and Trajanoski, Z. Analysis of DNA microarray data. Curr. Top. Med. Chem. 4 (13): 1357-1370, 2004.

Hager, J. Making and using spotted DNA microarrays in an academic core laboratory. Methods Enzymol. 410:135-168, 2006.

Handcock, M.S. and Morris, M. Relative distribution methods in the social sciences, 213228. New York, NY: Springer-Verlag, 1999.

Hao, L. and Naiman, D.Q. Quantile regression. Thousand Oaks, CA: Sage Pubns., 2007.

Hardiman, G. and Carmen, A. DNA biochips - past, present and future: an overview. In Biochips as pathways to drug discovery, ed. G. Hardiman and A. Carmen, 1-13. Boca Raton, FL: CRC Pr., 2006.

Hariharan, R. The analysis of microarray data. Pharmacogenomics 4 (4): 477-497, 2003.

Hatfield, G.W., Hung, S.P. and Baldi, P. Differential analysis of DNA microarray gene expression data. Mol. Microbiol. 47 (4): 871-877, 2003. 
He, Y.D. Genomic approach to biomarker identification and its recent applications. Cancer Biomarkers: Section A Dis. Markers 2 (3-4): 103-133, 2006.

Hoheisel, J.D. Microarray technology: beyond transcript profiling and genotype analysis. Nat. Rev. Genet. 7 (3): 200-210, 2006.

Huang, E., S. Ishida, J. Pittman, H. Dressman, A. Bild, M. Kloos, M. D’Amico, R.G. Pestell, M. West, and J.R. Nevins. Gene expression phenotypic models that predict the activity of oncogenic pathways. Nat. Genet. 34 (2): 226-230, 2003.

Jayapal, M. and Melendez, A.J. DNA microarray technology for target identification and validation. Clin. Exp. Pharmacol. Physiol. 33 (5-6): 496-503, 2006.

Kerns, R.T., Zhang, L. and Miles, M.F. Application of the S-score algorithm for analysis of oligonucleotide microarrays. Methods 31 (4): 274-281, 2003.

Koenker, R. Quantile regression. West Nyack, NY: Cambridge Univ. Pr., 2005.

Koenker, R. and Bassett, G. Regression quantiles. Econometrica 46 (1): 33-50, 1978.

Kohavi, R., and F. Provost. Glossary of terms. Mach. Learn. 30 (2/3): 271-274, 1998.

Krajewski, P. and Bocianowski, J. Statistical methods for microarray assays. J. Appl. Genet. 43 (3): 269-278, 2002.

Kramer, J.A. Overview of the tools for microarray analysis: transcription profiling, DNA chips and differential display. In Introduction to bioinformatics: a theoretical and practical approach, ed. S.A. Krawetz and D.D. Womble, 637-663. Totowa, NJ: Humana Pr., 2003. 
Kreil, D.P. and Russell, R.R. There is no silver bullet - a guide to low-level data transforms and normalisation methods for microarray data. Briefings Bioinf. 6 (1): 86-97, 2005.

Kubat, M., R.C. Holte, and S. Matwin. Machine learning for the detection of oil spills in satellite radar images. Mach. Learn. 30 (2/3): 195-215, 1998.

Larsson, O., Wennmalm, K. and Sandberg, R. Comparative microarray analysis. OMICS 10 (3): 381-397, 2006.

Lewis, D.D., and W.A. Gale. A sequential algorithm for training text classifiers. In SIGIR '94 proceedings of the seventeenth annual international ACM-SIGIR conference on research and development in information retrieval, ed. W.B. Croft and C.J. van Rijsbergen, 3-12. New York, NY: Springer-Verlag, 1994.

Li, W. and Yang, Y. Zipf's law in importance of genes for cancer classification using microarray data. J. Theor. Biol. 219 (4): 539-551, 2002.

Liang, Y. and Kelemen, A. Associating phenotypes with molecular events: recent statistical advances and challenges underpinning microarray experiments. Funct. Integr. Genomics 6 (1): 1-13, 2006.

Loader, C. Local regression and likelihood. New York, NY: Springer-Verlag, 1999.

Loader, C. Smoothing: local regression techniques. In Handbook of computational statistics: concepts and models, ed. J.E. Gentle, W. Hardle and Y. Mori, 539-563. New York, NY: Springer, 2004.

Mandruzzato, S. Technological platforms for microarray gene expression profiling. $A d v$. Exp. Med. Biol. 593:12-18, 2007. 
Nadon, R. and Shoemaker, J. Statistical issues with microarrays: processing and analysis. Trends Genet. 18 (5): 265-271, 2002.

Nanni, L., Romualdi, C., Maseri, A. and Lanfranchi, G. Differential gene expression profiling in genetic and multifactorial cardiovascular diseases. J. Mol. Cell. Cardiol. 41 (6): 934-948, 2006.

National Research Council. Toxicity testing in the $21^{\text {st }}$ century: a vision and a strategy. Washington, DC: National Academies Pr., 2007.

Neal, S.J. and Westwood, J.T. Optimizing experiment and analysis parameters for spotted microarrays. Methods Enzymol. 410:203-221, 2006.

Ogawa, K., Murayama, S. and Mori, M. Predicting the tumor response to radiotherapy using microarray analysis. Oncol. Rep. 18 (5): 1243-1248, 2007.

Pan, W. A comparative review of statistical methods for discovering differentially expressed genes in replicated microarray experiments. Bioinformatics 18 (4): 546-554, 2002.

Parrish, R.S. and Delongchamp, R.R. Normalization of microarray data. In DNA microarrays and related genomics techniques: design, analysis and interpretation of experiments, ed. D.B. Allison, G.P. Page, T.M. Beasley and J.W. Edwards, 9-28. Boca Raton, FL: CRC Pr., 2006.

Petersen, D.W. and Kawasaki, E.S. Manufacturing of microarrays. Adv. Exp. Med. Biol. 593:1-11, 2007.

Quackenbush, J. Microarray data normalization and transformation. Nat. Genet. Suppl. 32:496-501, 2002. 
Reimers, M. Statistical analysis of microarray data. Addict. Biol. 10 (1): 23-35, 2005.

Roesok, O. and Sioud, M. Discovery of differentially expressed genes: technical considerations. Methods Mol. Biol. 360:115-129, 2007.

Sabatti, C. Statistical issues in microarray analysis. Curr. Genomics 3 (1): 7-12, 2002.

Sartor, M.A., Medvedovic, M. and Aronow, B.J. Microarray data normalization: the art and science of overcoming technical variance to maximize the detection of biologic variance. In A beginner's guide to microarrays, ed. E.M. Blalock, 151-178. Norwell, MA: Kluwer Academic Publishers, 2003.

Saviozzi, S. and Calogero, R.A. Microarray probe expression measures, data normalization and statistical validation. Comp. Funct. Genomics 4 (4): 442-446, 2003.

Saviozzi, S., Iazzetti, G., Caserta, E., Guffanti, A. and Calogero, R.A. Microarray data analysis and mining. Methods Mol. Med. 94:67-90, 2004.

Seber, G.A. and Lee, A.J. Linear regression analysis, $2^{\text {nd }}$ ed. Hoboken, NJ: John Wiley and Sons, 2003.

Slonim, D.K. From patterns to pathways: gene expression data analysis comes of age. Nat. Genet. Suppl. 32:502-508, 2002.

Steinhoff, C. and Vingron, M. Normalization and quantification of differential expression in gene expression microarrays. Briefings Bioinf. 7 (2): 166-177, 2006.

Stolovitzky, G. Gene selection in microarray data: the elephant, the blind men and our algorithms. Curr. Opin. Struct. Biol. 13 (3): 370-376, 2003. 
Tadesse, M.G. and Ibrahim, J.G. A Bayesian hierarchical model for the analysis of Affymetrix arrays. Ann. N.Y. Acad. Sci. 1020:41-48, 2004.

Taylor, R.F. Microarrays. In Kirk-Othmer encyclopedia of chemical technology, $5^{\text {th }}$ ed., ed. A. Seidel, Vol. 16, 380-394. Hoboken, NJ: Wiley, 2006.

Tusher, V.G., R. Tibshirani, and G. Chu. Significance analysis of microarrays applied to the ionizing radiation response. Proc. Natl. Acad. Sci. U.S.A. 98 (9): 5116-5121, 2001.

Tusher, V.G., R. Tibshirani, and G. Chu. Significance analysis of microarrays. U.S. Patent Application 20020019704, filed on Feb. 14, 2002.

Vedoy, C.G., Bengtson, M.H. and Sogayar, M.C. Hunting for differentially expressed genes. Braz. J. Med. Biol. Res. 32 (7): 877-884, 1999.

Vietor, E. and Huber, L.A. In search of differentially expressed genes and proteins. Biochim. Biophys. Acta 1359 (3): 187-199, 1997.

Villeneuve, D.J. and Parissenti, A.M. The use of DNA microarrays to investigate the pharmacogenomics of drug response in living systems. Curr. Top. Med. Chem. 4 (13): 1329-1345, 2004.

Vo-Dinh, T. Biosensors and biochips. In BioMEMS and biomedical nanotechnology, ed. M. Ferrari, Vol. IV, 3-20. New York, NY: Springer, 2006.

Wang, H. and He, X. Detecting differential expressions in GeneChip microarray studies: a quantile approach. J. Am. Stat. Assoc. 102 (477): 104-112, 2007.

Wolkenhauer, O., Moller-Levet, C. and Sanchez-Cabo, F. The curse of normalization. Comp. Funct. Genomics 3 (4): 375-379, 2002. 
Yang, D., Zakharkin, S.O., Page, G.P., Brand, J.P.L., Edwards, J.W., Bartolucci, A.A. and Allison, D.B. Applications of Bayesian statistical methods in microarray data analysis. Am. J. Pharmacogenomics 4 (1): 53-62, 2004.

Yang, I.V. Use of external controls in microarray experiments. Methods Enzymol. 411:50-63, 2006.

Yu, K., and M.C. Jones. Local linear quantile regression. J. Am. Stat. Assoc. 93 (441): 228-237, 1998.

$\mathrm{Yu}, \mathrm{K} ., \mathrm{Lu}, \mathrm{Z}$. and Stander, J. Quantile regression: applications and current research areas. The Statistician 52 (3): 331-350, 2003.

Zhang, D., Wells, M.T., Smart, C.D. and Fry, W.E. Bayesian normalization and identification for differential gene expression data. J. Comput. Biol. 12 (4): 391-406, 2005. 